\title{
AN INTEGRATIVE FRAMEWORK ON THE NATURE, ANTECEDENTS AND OUTCOMES OF PERCEIVED DISCRIMINATION IN THE WORKPLACE
}

\author{
By \\ Ma. Socorro G. Diego-Mendoza
}

\begin{abstract}
A thesis
submitted to the Victoria University of Wellington

in fulfillment of the requirements for the degree of

Doctor of Philosophy in Psychology
\end{abstract}

Victoria University of Wellington

Wellington, New Zealand

2010 


\begin{abstract}
This thesis aimed to advance the knowledge on perceived discrimination (PD) from targets' perspectives and address gaps in research. Study 1 reports a meta-analysis examining correlates of PD across 63 studies involving 37,790 participants. Findings showed that greater PD was associated with increased race/ethnic identification, negative well-being, and attitudes favouring affirmative action (AA). Greater PD was also related to low job satisfaction, low self-esteem and low socio-economic status (SES). The strongest relationship was found between PD and job satisfaction, followed by negative well-being, race/ethnic identity, self-esteem, AA attitudes and SES. The negative correlation between PD and ethnic identity was moderated by type of measurement, research context and type of sample. Similarly, the link between SES and PD was moderated by research context and type of sample. The meta-analysis also identified major research gaps that served as the jump off point for the next studies.
\end{abstract}

Consequently, a three-component research framework for the study of PD was proposed and tested in three studies. The components were: 1) nature of PD, 2) antecedents of PD, and 3) outcomes of PD. Study 2 explored the first component through separate focus groups discussions participated by immigrants from China (5), India (5), Philippines (5), Germany (4) and Zimbabwe (4). Through thematic analysis, a 4-quadrant model emerged from the themes.

In Study 3-A, three PD scales that fit the focus groups' model were constructed and collectively named as Immigrants' Perceptions of Discrimination 
in the Workplace Scales (IPDWS). The scales' psychometric properties were tested using 155 employed immigrants. Ten factors emerged and converged into two higher order factors - Job-Entry and On-the-Job. Study 3B examined job and psychological well-being outcomes using the same immigrant sample. Findings showed that high PD (in career advancement, compensation and equal treatment in policies factors) predicted low job satisfaction, high PD (in career advancement and derogation) predicted low affective commitment, high PD (exclusion factor) predicted high turnover intentions, and high PD (derogation factor) predicted less life satisfaction and greater psychological distress.

Finally, Study 4 examined proximal (age, gender, ethnicity and birthplace) and distal (organizational demographics, perceptions of justice and practices) antecedents of PD through 540 employees using Employee Perceptions of Discrimination in the Workplace Scales (a modified version of IPDWS). Findings showed that increasing age and lower income predicted lower PD (nonrecognition of education and experience). Conversely, increasing age predicted higher PD in career advancement. Moreover, greater PD (in career advancement) occurred in large organizations and the public sector (work dynamics). For organizational justice, higher procedural justice predicted lower PD (career advancement factor), interpersonal justice predicted lower PD (work dynamics and derogation factors), and informational justices predicted low PD (recognition of qualifications, career advancement, and work dynamics factors). As for organizational practices, higher employee-orientation predicted low PD (work dynamics) and higher innovation-orientation predicted low PD (career path 
factor). Overall, distal antecedents were better predictors of PD than the proximal antecedents. In summary, the research findings contribute to the general discrimination and organizational literatures and offer suggestions to policymaking bodies. 


\section{ACKNOWLEDGEMENTS}

I would like to express my sincerest gratitude to the following people and institutions to whom I am deeply indebted,

Thanks to my three supervisors, Dr. Ronald Fischer, Prof. Colleen Ward and Dr. Astrid Podsiadlowski, for their endless encouragement, patience, advices during the course of my study. Special thanks to you Ron for introducing me the magical world of quantitative analysis. Thank you for being a brilliant mentor and a friend. I would have not survived my $\mathrm{PhD}$ without you. Thank you Colleen for sharing your wisdom, you always redirected me to the right path by holding a light for me at the end of a dark tunnel. Thank you Astrid for being my first mentor. I would have gone back home if not for your patient and dedicated guidance during the initial year of my PhD study and for your continued interest in my progress even after you left VUW.

Thank you to Prof. Charles T. Hill, Dr. Yolanda Mendoza and Pip Collie who patiently and tirelessly proofread my drafts despite the very short notice.

Thanks to Shona de Sain and the staff of Faculty of Science for opening doors for me, especially in the final completion of my thesis; to Camilla Swan of Education New Zealand and Barry Lewis of the VUW Scholarship Office who took good care of my financial needs while I was at VUW; to Ngaire Lavery and Jebi Jayapalan of the School of Psychology who looked after my administrative needs at SoP.

Thanks to New Zealand Federation of Ethnic Councils and the representatives of the ethnic associations in New Zealand, who assisted me in recruiting participants for my studies.

Thanks to the Filipino students of VUW, to the Filipino community in Wellington, to my foster families in Lower Hutt (and to Aben in the US) and to Filipino friends in Palmerston North - who helped me recruit participants, distribute and collect questionnaires, gather reference materials, or just simply made me feel at home in Aotearoa, Maraming Salamat Po! 
My sincerest gratitude to my examiners, Prof. James Liu (VUW), Prof. Stuart C. Carr (Massey University) and Prof. Dharm P. S. Bhawuk (University of Hawaii at Manoa) who provided valuable insights and comments to my thesis.

Thanks to my 'family' in the Cross-cultural Lab. My $\mathrm{PhD}$ life was amazingly fun because of you - either during busy days and late nights in Kelburn or when we are happily hopping around the world! Special thanks to Diana, Katja and Vivian - for answering my even most mundane questions about stats; to Larissa and Elena - for taking care of me amidst their own busy lives; to Mel, Sammyh and Taciano - for sharing their knowledge and resources; and to the past and current members of the CACR postgraduate student network with whom I had the privilege to build lasting friendships, you have helped me in so many ways. All of you will remain important in my life even beyond $\mathrm{PhD}$.

To my family and close relatives in the Philippines for your quiet encouragement, prayers and enduring love, Miss ko na kayo!

Last but not least, to my beloved husband Arnel, thanks for your endless support and encouragements, for patiently looking after me while I work, and for patiently bringing back all the $\mathrm{PhD}$ materials that I have thrown away. This $\mathrm{PhD}$ journey has led me back to you for a reason - you are the reason.

This doctoral research has been supported by:

New Zealand International Doctoral Research Scholarships (NZIDRS) (Education New Zealand)

Victoria PhD Submission Scholarship (2009)

(Victoria University of Wellington)

\section{Without you this thesis would have not been possible. THANK YOU!}

To my participants who freely shared their time and thoughts in broadening the understanding of the intricate nature of Perceived Discrimination.

This thesis is for you. 
Table of Contents

Title

Page

Abstract....................................................... ii

Acknowledgements ........................................ v

Table of Contents........................................... vii

List of Tables................................................ xii

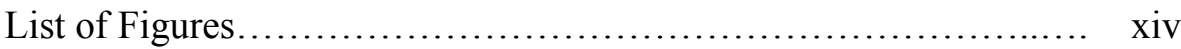

Chapter 1: Introduction ........................................ 15

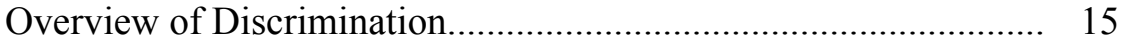

Discrimination Research: Current Trends............................ 17

Overt vs. Covert Forms of Discrimination....................... 18

Perpetrators vs. Targets.................................................... 20

Economic vs. Psychological Effects of Discrimination..... 22

Actual Experience vs. Perceived Discrimination (PD)..... 23

Gaps in Discrimination Research............................................... 24

Overview of the Chapters.......................................................... 25

\section{Chapter 2: Antecedents and Outcomes of Perceptions of}

Discrimination: A Meta-Analysis (Study 1).................... 28

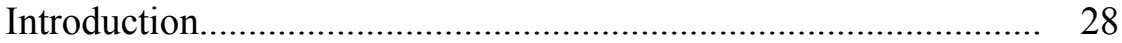

Meta-Analysis as a Research Methodology........................... 29

Research Domains........................................................... 31

Social Psychology ........................................................... 32

Personality-Related..................................................... 37

Health Psychology .......................................................... 40

Organizational Psychology............................................ 43

Discrimination and Demographic Variables......................... 45

Moderating Effects of Contextual Variables.......................... 47

Study Characteristics................................................ 48

Participants' Characteristics.......................................... 49

Summary of Proposed Hypotheses...................................... 50

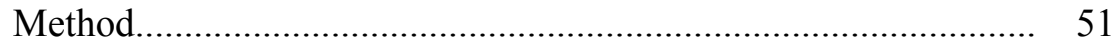

Article Identification and Selection..................................... 51

Coding of Variables......................................................... 52

Computation of Effect Sizes and Moderator Analysis............ 61

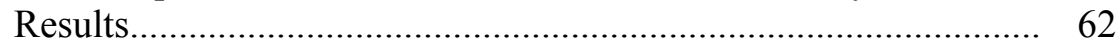

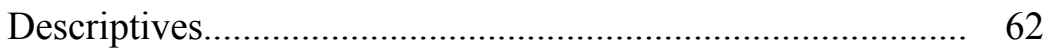

Moderator Analyses........................................................ 63

Discussion......................................................................... 68

The Overall Relationship of PD with its Correlates............... 68

The Role of Contextual Variables........................................ 71

Limitations of the Study ...................................................... 75

Suggestions for Future Research...................................... $\quad 77$

Summary/Conclusion........................................................ 80 


\section{Chapter 3: A Framework for the Study of Perceived}

Discrimination in the Workplace..................................... 81

Introduction

Access and Treatment Discrimination.................................. 82

Formal and Informal Employment Discrimination................. 84

Objective vs. Subjective Measures of Discrimination in the

Workplace............................................................ 84

Antecedent-Outcome Framework of PD in the Workplace........... 90

Conceptualization of PD using Target's Perspectives............. 92

Antecedents of Perceived Discrimination .............................. 94

Proximal Antecedents................................................ 95

Distal Antecedents..................................................... 99

Consequences of Perceived Discrimination.......................... 104

Organizational Outcomes........................................... 104

Psychological Well-being............................................. 105

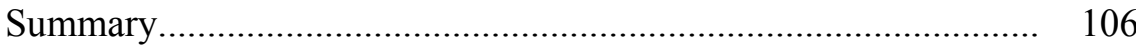

Chapter 4: The Nature of PD from the Immigrants' Perspective:

A Qualitative Study (Study 2)......................................... 108

Introduction............................................................................ 108

Workplace Discrimination in the Context of Globalization.... 109

Global Immigration: Changing Trends................................... 110

New Zealand Immigration: Past \& Current Trends................. 115

History of Immigration............................................ 115

Total Population............................................................. 117

Immigrant Population................................................... 118

Emerging Barriers to Successful Immigrant Settlement in New Zealand...................................................... 120

Are New Zealand Workplaces Discriminatory?............. 127

Study 2: Focus Groups Discussions............................................... 132

Specific Objectives.......................................................... 132

Focus Groups Discussion as a Research Method................... 133

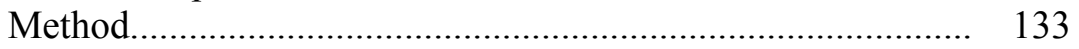

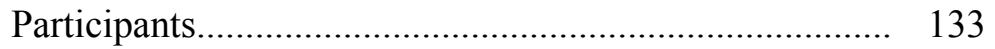

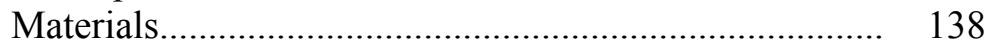

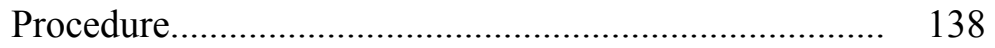

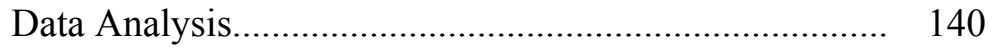

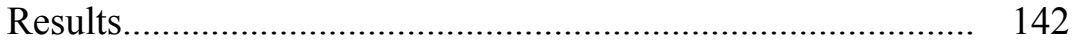

Overview of NZ Organizations.................................. 142

Culture-Specific Barriers to Positive Employment Outcomes................................................................. 144

Culture-Specific Facilitators to Positive Employment Outcomes........................................................... 146

NZ-Specific Pre-Hiring Barriers to Positive Employment Outcomes.......................................... 148

NZ-Specific On-the-job Barriers to Positive Employment Outcomes......................................... 152

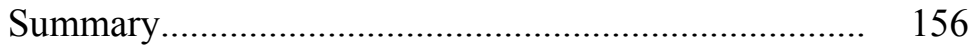

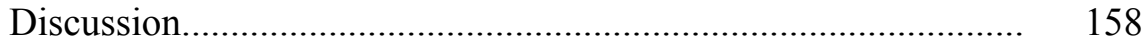

Limitations of the Study.................................................... 162 
Suggestions for Future Research........................................ 164

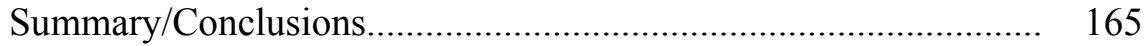

\section{Chapter 5: Scale Construction and Validation: IPDWS}

(Study 3-A)............................................................ 167

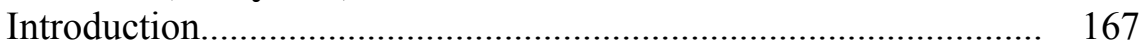

Review of Related Literature on PD Measurements..................... 168

Existing Measurements on Race or Ethnicity-Related

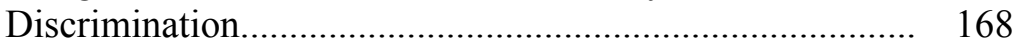

Personal vs. Group Perceptions......................................... 170

Group Identification: Race or Ethnicity.............................. 174

Lateral, Vertical and Systemic PD..................................... 178

Frequency of the Discriminatory Perceptions or Experience.. 181

Measurement Issues..................................................... 186

Racism vs. Race/Ethnic Discrimination...................... 187

Single Item Measures.................................................... 188

Workplace-Related Scales......................................... 188

Immigrants' Perceptions............................................. 189

Methodology ......................................................................... 193

Item Development........................................................... 193

Instrument Refinement and Initial Psychometric Properties... 196

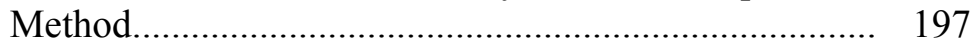

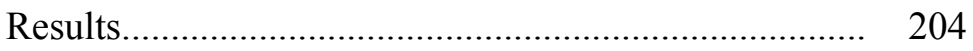

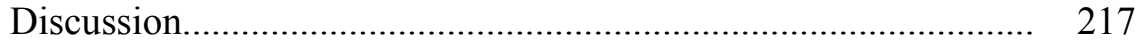

Exploratory Factor Analyses Output................................ 218

Intercorrelations Output................................................. 222

Limitations of the Study................................................... 223

Suggestions for Future Study........................................... 224

Summary/Conclusions............................................................ 225

\section{Chapter 6: Outcomes of PD: Organizational \& Psychological}

Well-being (Study 3-B)...................................................... 226

Introduction...................................................................... 226

Perceived Discrimination and Organizational Outcomes............. 228

Job Satisfaction................................................................ 229

Organizational Commitment............................................... 231

Turnover Intention........................................................ 233

Perceived Discrimination and Psychological Well-being............ 236

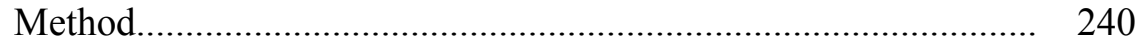

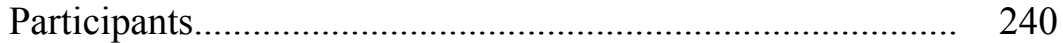

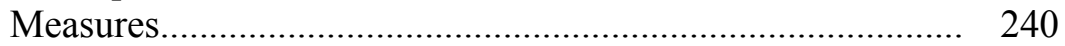

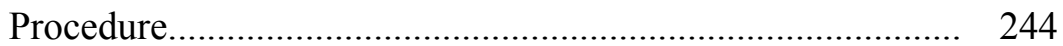

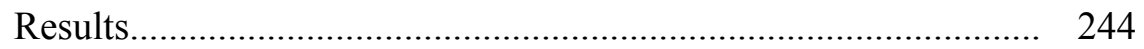

Descriptives.................................................................. 244

Intercorrelations.......................................................... 246

Regression Analyses.................................................... 248

Discussion......................................................................... 254

Pre-employment Perceptions (Job-Entry) and Outcomes.... 256

During employment (On-the-Job) Perceptions and

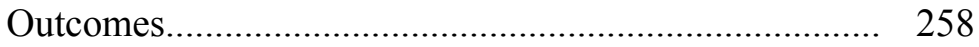


Limitations of the Study................................................... 262

Suggestions for Future Research...................................... 263

Summary/Conclusions.................................................................. 264

Chapter 7: Antecedents of Perceived Discrimination (Study 4)... 266

Introduction........................................................................... 266

Factors Affecting PD in the Workplace.................................... 270

Proximal Influences....................................................... 271

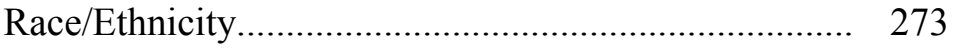

Birthplace............................................................ 274

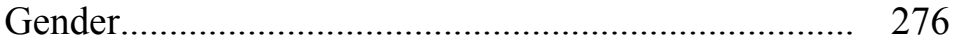

Age

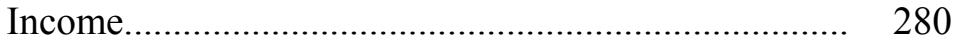

Distal Influences........................................................ 281

Perceptions of Organizational Justice........................ 282

Perceptions of Organizational Practices..................... 284

Size and Sector of Organizations............................... 287

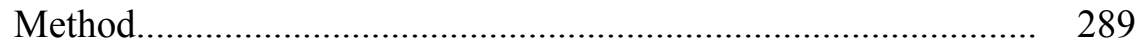

Participants.................................................................. 289

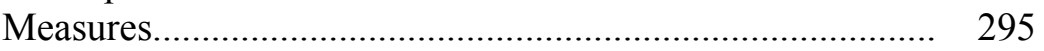

Procedure.............................................................. 304

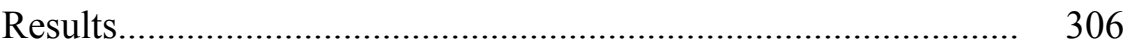

Descriptives............................................................ 306

Hierarchical Regression Analyses................................... 306

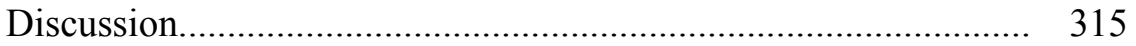

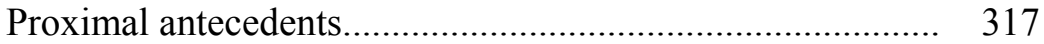

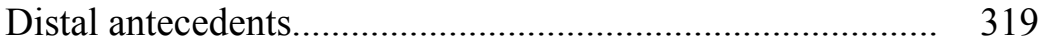

Limitations................................................................. 328

Suggestions for future research..................................... $\quad 329$

Summary/Conclusion............................................................. 331

Chapter 8: Discussion and Conclusions.......................................... 334

Contribution to the General Discrimination Literature................... 335

Contribution to Organizational Psychology ................................... 341

The Nature and Measurement of PD.................................. 342

The Nature of PD....................................................... 342

Measurements of PD................................................ 345

Summary................................................................ 349

Antecedents of Perceived Discrimination.......................... $\quad 350$

Proximal Antecedents............................................. 351

Distal Antecedents..................................................... 352

Summary................................................................ 354

Perceived Discrimination and Outcomes.......................... 356

Job Outcomes........................................................ 356

Well-being Measures............................................... 358

Summary .......................................................... 359

Limitations and Future Research.............................................. 362

Implications and Practical Applications for Organizations and

Policy Makers...................................................................... 363

Conclusions............................................ 367 


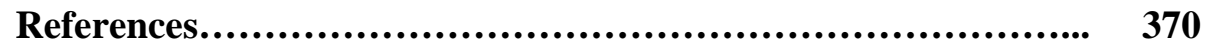

Appendices........................................................... 417

A. Focus Groups Discussions Schedule............................. 417

B. Perceptions of Discrimination Scales - Initial Items During Item Development Stage ................................. 418

C. Immigrants' Perceptions of Discrimination in the Workplace

Scales (IPDWS) - Pilot Version.............................. 420

D. Employee Perceptions of Discrimination in the Workplace

Scales (EPDWS) - Initial Items......................... 422

E. Information Sheet - Study 2 (Focus Groups Discussion)..... 423

F. Information Sheet - Study 3 (Survey 1) .................... 425

G. Information Sheet - Study 4 (Survey 2).................. 427 


\section{List of Tables}

$\underline{\text { Number }}$

$\underline{\text { Title }}$

$\underline{\text { Page }}$

Table 2.1: Overview of studies on the correlates of Perceptions of

Discrimination.

Table 2.2: $\quad$ Meta-analytic relationships between PD and

Correlates

Table 2.3: Summary of Significant Moderators.

Table 4.1: Distribution of New Zealand Population.

Table 4.2:

Percentage Increase in the Population of People Born Overseas.....

Table 4.3: Survey Participants According to Region of Origin, SMC \& Professionals.

Table 4.4: Demographic Details of Focus Groups Discussion (FGD) Participants.

Table 4.5: Immigrants' Perceptions of Workplace Discrimination in New Zealand

Table 5.1: $\quad$ A Sampling and Evaluation of Existing Scales for Measuring Race/Ethnicity-Related Perceptions of Discrimination.

Table 5.2:

IPDWS' Fit in the FGD Model.

Table 5.3:

Results of the Content Validation of Initial Scale Items.

196

Table 5.4

Participants Demographics ( $\mathrm{N}=155)$...

199

Table 5.5:

Organizations' Demographics.

200

Table 5.6:

Participants' Ethnic Background

201

Table5.7:

Work

Table 5.8:

Factor Loadings and Internal Consistency for Work Conditions.

Table 5.9: $\quad$ Factor Loadings and Internal Consistency for Work

Relationships.

Table 5.10: Zero-order Correlations Among Factors in the IPDWS (Raw Score).

Table 5.11: Pearson Correlations Among Factors in the IPDWS and English Proficiency....

Table 5.12: Factor Loadings and Internal Consistency for the Second Order Factors.

Table 6.1: Descriptive Statistics and Reliability Coefficients of Predictor and Outcome Variables.

Table 6.2: Correlation Matrix for the Immigrants' Perceptions of Discrimination in the Workplace Scale and Dependent Variables.

Table 6.3: $\quad$ Standardized Coefficients ( $(B)$ in Hierarchical Regressions for the Prediction of Organizational Outcomes.

Table 6.4: $\quad$ Standardized Coefficients ( $(B)$ in Hierarchical Regressions for the Prediction of Psychological-Well being Outcomes. 
Table 7.1: $\quad$ Employee Participants’ Personal Demographics.......... 290

Table 7.2: $\quad$ Immigrants' Countries of Birth .................................... 292

Table 7.3: $\quad$ Ethnic Backgrounds of New Zealand-Born Participants............................................................... 294

Table 7.4: $\quad$ Organizations' Demographics....................................... 295

Table 7.5: Factor Loadings, Variances and Reliability

Coefficients for Finding Work................................... 299

Table 7.6: $\quad$ Factor Loadings, Variances and Reliability Coefficients for Work Conditions ............................... 300

Table 7.7: $\quad$ Factor Loadings, Variances and Reliability Coefficients for Work Relationships........................... 301

Table 7.8: Descriptive Statistics for the PD Factors and Participants Demographics........................................ 307

Table 7.9: $\quad$ Psychometric Properties of PD Distal Antecedents...... 308

Table 7.10: $\quad$ Standardized Coefficients $(ß)$ in Hierarchical Regressions for the Prediction of Job-Entry Perceptions of Discrimination

Table 7.11: $\quad$ Standardized Coefficients $(\Omega)$ in Hierarchical Regressions for the Prediction of On-the-Job Perceptions of Discrimination.

Table 7.12: $\quad$ Summary of Significant Predictors of PD Factors (ß-values)................................................................ 314

Table 8.1: $\quad$ Perceptions of Workplace Discrimination ................... 344

Table 8.2: $\quad$ IPDWS' Fit in the FGD Model................................... 346

Table 8.3: IPDWS' (10 factors) Fit in the FGD Model................. 346

Table 8.4: $\quad$ EPDWS (4 factors) Fit in the FGD Model................... 348 


\section{List of Figures}

Number

$\underline{\text { Title }}$

$\underline{\text { Page }}$

Figure 3.1: Antecedents-Consequence Research Framework on

Perceived Discrimination in the Workplace..

Figure 3.2: The Systems Model of Discrimination (SMD) by

Gelfand et al. (2005).....

Figure 4.1: Migrant Population as a Percentage of Total

Population

Figure 4.2: Annual Median Income (NZ\$) of Different Ethnic

Groups

Figure 4.3: Distribution of NZ Population by Income

(2001).

Figure 4.4: Income Distribution of Survey Participants

Figure 5.1: Existing Measurement Approaches for Ethnicity/Race Related Discrimination.

Figure 6.1 Summary of Significant Findings Between PD and Outcomes.

Figure 8.1: Antecedent-Consequence Research Framework on the study of Perceived Discrimination in the Workplace.

Figure 8.2: Summary of Finding on the Nature of PD. 350

Figure 8.3: Significant Antecedent/Predictor of PD (Mixed Samples)....

Figure 8.4: Significant Outcomes Predicted by PD (Immigrant Sample). 


\section{Chapter 1}

\section{INTRODUCTION}

\section{Overview of Discrimination}

The world continues to witness the recurring instances of discrimination ranging from its worst forms to the subtlest. Due to its pervasiveness, discrimination has been a focus of moral, political and empirical discourse for at least a century. From the moral perspective, discrimination is discussed around the issues of equality and inequality. Allport (1954, cited in Dovidio \& Gaertner, 1986) defined discrimination as unfair negative behaviours that hinder "individuals or groups of people equality of treatment" (p.51). Inequality in Allport's definition is related to Therborn's (2006) existential inequality which he defined as inequality in relation to freedom and respect. Race/ethnicity, gender, the caste system in India, slavery and servitude in Africa and America represent examples of existential inequality.

The political discourse on discrimination gave rise to legislations that prohibited discriminatory acts. The United States historically played a significant role in the creation of the United Nations and in the drafting of the Universal Declaration of Human Rights (UDHR) ${ }^{1}$. In fact, the UDHR was partially patterned from the U.S. Bill of Rights (National Coordinating Committee for UDHR, 1998; Wilson, 1988). One of the earliest antidiscriminatory legislations passed in the United States was the Civil Rights Act of 1866 that gave Black people equal rights with that of White people (Dovidio \& Gaertner, 1986).

\footnotetext{
${ }^{1}$ The majority discrimination research originates from the US.
} 
Member countries of the United Nations have active human rights legislations based on the UDHR that was adopted as a covenant in the United Nations General Assembly held on the $10^{\text {th }}$ of December 1948. Similarly, the New Zealand has its own version of human rights legislation, the Human Rights Act 1993, which prohibits discrimination on the basis of colour, race, ethnic or national origins, sex, age, sexual orientation, marital status, religious belief, ethical belief, disability, political opinion, employment status and family status (Human Rights Commission [HRC], 2005). According to this law, "discrimination is only considered unlawful if it involves a ground AND an area" (HRC, 2000, p. 9). Thus, the different grounds of discrimination are colour, race, ethnic or national origins, sex, age, sexual orientation etc. Further, the considered main areas of discrimination are employment and preemployment, supply of goods and services, education, access to public places, vehicles and facilities, and housing and accommodation.

Empirical work on discrimination increased simultaneously with the heightened political interest to eliminate discriminatory acts. Early scholarly studies on discrimination provided information for legislations intended to control race-related discrimination and to increase acceptance of diversity. Reasonably, discrimination has been a central interest in multi-disciplinary research for the past half a century. Early socio-psychological studies of discrimination were geared towards the reduction of social problems (Branscombe, Schmitt \& Harvey, 1999). The continuing interest in discrimination research is brought about by the evolving nature of discrimination despite active social, political and legal control measures. 
Discrimination, as a topical issue, continues to generate new thinking and research from various perspectives and different research domains such as sociology, social psychology, acculturation research, organizational psychology, management and economic research. As a consequence, separate disciplines have focused their work on similar grounds of discrimination like race/ethnic discrimination (e.g. Finch, Kolody \& Vega, 2000; Konrad \& Spitz, 2003; Landrine \& Klonoff, 1996; Phinney, Madden, \& Santos, 1998; Rodin, Price, Bryson, \& Sanchez, 1989; Rugiero \& Taylor, 1995; Verkuyten, 1998), gender discrimination (e.g. Halpert, 1993; Hickman, 1993; Kirchmeyer, 2002; Levitin, Quinn, \& Staines, 1971; Shaffer, Joplin, Bell, Lau, \& Oguz, 2000; Trentham \& Larwood, 1998; Wilson, 1994), age discrimination (e.g. Yearta \& Warr, 1995; O’Boyle, 2001) and disability (e.g. Balser, 2000; Premeaux, 2001). Due to the varying research domains and different theoretical approaches generated from the studies, Dipboye and Collela's (2005) issued a call for the reintegration of the disparate bodies of theoretical models and methodologies in discrimination research specifically in area of workplace discrimination.

\section{Discrimination Research: Current Trends}

The discrimination literature is continually evolving as the nature of discrimination changes its form. Gradually, the trend in scholarly research on discrimination is changing from overt to covert forms of discrimination, from perpetrators to victims/targets of discrimination, from a focus on actual events to the interpretation of these events in terms of perceived discrimination and 
from economic measurements to psychological approaches in understanding the discrimination process.

\section{Overt vs. covert forms of discrimination.}

Acts of overt discrimination can range from mild forms such as verbal abuse to more severe forms including genocide (Marger, 2000). Historical evidence shows that as early as the 1900 's, discrimination was manifested in the form of universally institutionalized racism of white supremacy, the patriarchal society and Euro-American colonialism (Therborn, 2004). Moreover, from the late $20^{\text {th }}$ century up to present is characterized by continuing worldwide struggles against ethnicity, gender and sexual orientation discrimination. On top of these struggles is the mounting racism against immigrants in Europe.

The recent economic integration of different countries, brought about by increased globalization, has paved the way for yet another target of discriminatory attitudes - the immigrants. Akin to ethnic minorities, immigrants possess characteristics that serve as bases for their exclusion like skin color, language differences, physical features and cultural traits that are different from the native-born group (Phiney et al., 1998; Westhoff, 1993). As a consequences, immigrants generally face an array of challenges including experiencing discrimination (International Organization for Migration [IOM], 2005; Ward \& Leong, 2006).

Reports continue to show that instances of overt discriminatory practices are decreasing after being declared as unlawful, however new and less visible forms have taken its place (International Labour Organization [ILO], 2003). A review of discrimination studies by Dipboye and Collela 
(2005) revealed that while overt or blatant discriminatory acts are at present considered illegal, discrimination continues to be relevant and has taken covert forms that are less explicit, unconscious and sometimes embedded in organizational structures. Most covert forms of discrimination are observed through interaction biases (Dovidio \& Hebl, 2005) such as managers being less friendly and helpful with target groups (Hebl, Foster, Mannix, \& Dovidio, 2002). The resulting impact of these biased behaviours towards target groups are reduced support and quality of mentorship (Raggins, 1999) eventually affecting minority groups' efficiency and performance (Dovidio \& Hebl, 2005).

Specifically, subtle forms of ethnic discrimination and racism continue to be observed and experienced by target groups despite individual, societal and legal pressures (Dovidio \& Gaertner, 1986). Discrimination continues to persist along racial and ethnic lines despite attempts to curb discriminatory behaviours (Dymski, 2001). In a recent report by the Equal Employment Opportunity Commission ([EEOC], 2008) in the United States, race-related and national origin-related discrimination complaints comprised $37.0 \%$ and $11.4 \%$ of the total cases respectively. These figures have shown consistent increasing trends in the EEOC data for a 10-year period starting 1997 to 2007, with a higher increase for national origin-related discrimination. Similarly, the New Zealand Human Rights Commission's (2007) report showed that $34.8 \%$ of human rights violation complaints were race related. Race-based complaints include discrimination on the grounds of race, colour, ethnic or national origins, racial harassment and racial disharmony. This figure is the highest percentage in comparison to other form of complaints. Furthermore, race- 
related complaints have shown an increasing trend, from $13.0 \%$ in 2002 to $17.1 \%$ in 2007 (HRC, 2002; 2007). Therefore, it is clearly evident that ethnicity- or race-related forms of discrimination are still very much a reality and very relevant in the modern age.

\section{Perpetrators vs. targets.}

The general literature on discrimination is concentrated either on perpetrators or targets of discrimination. The perpetrators of discrimination are those who undertake the acts of discrimination (see Brief, Dietz, Cohen, Pugh, \& Vaslow, 2000; Petersen \& Dietz, 2005; Stewart \& Perlow, 2001; Trentham \& Larwood, 1998). The targets of discrimination, on the other hand, are those who experience or perceive the discriminatory acts (e.g. Balser, 2000; Deitch, Barsky, Butz, Chan, Brief \& Bradley, 2003; Ensher, Grant-Vallone, \& Donadson, 2001; Sanchez \& Brock, 1996). Documented usually as target groups are those who belong to minority groups including migrants, women, older people, gays and lesbians, and the disabled.

Traditionally, research efforts were focused on understanding the attitudes and rationale of perpetrators, that has been described as "psychology of the powerful" (Branscombe, et al., 1999, p.135) or the "psychology of the dominant groups" (Romero \& Roberts, 1998, p.641). From the point of view of scholars whose studies focus on perpetrators, discrimination is defined as "an unjustified negative behaviour toward members of a target group in the context of social interaction" (Dovidio \& Gaertner, 1986, p.6). Psychologists had conventional interest in the study of the cognitive, emotional and behavioural antecedents and consequences of constructs like stigma, 
stereotyping, prejudice and discrimination from the point of view of the dominant group (Harvey, 2006).

This led to an increased interest in the prejudice-discrimination relationship and attitude-behaviour research (see meta-analysis of Shutz \& Six, 1996). Prejudice is differentiated from discrimination such that the former is the negative belief or attitude towards certain groups of people with specific characteristics. Discrimination, on the other hand, is the behavioural manifestation that hinders a particular group of people's equal opportunity to resources (Marger, 2000). There have been inconsistent findings regarding this attitude-behaviour approach showing low correlations between attitudes and behaviour (e.g. McGuire, 1985) and prejudice has been found to be an inconsistent predictor of social discrimination (Shutz \& Six, 1996). In contrast to popular belief, therefore, prejudice does not always lead to discrimination and acts of discrimination by perpetrators are caused by factors other than prejudice (Dovidio \& Gaertner, 1986).

Gradually, interest in the "psychology of the oppressed groups" (Romero \& Roberts, 1998, p.641) or the targets of discrimination as the focal point of scholarly research has grown in recent years (see reviews by Crocker, Major, \& Steele, 1998; Romero \& Roberts, 1998; Deitch et al., 2003). The psychology of minorities was pointed out by Dovidio and Hebl (2005) as a future area of study. They called for research looking into the cognitions, emotions and behaviours of minorities in a way that portrays them as active players in social interactions. Ample focus has been accorded to particular members of target groups in previous studies, such as ethnic minorities and women (see review of Ibarra, 1993). In recent years, other stigmatized 
members of target groups have also caught the attention of scholars and legislators including those who are victims of discrimination related to age, religion, sexual preference, and disability. This thesis continues this line of research by focusing on the point of view of the targets as a contribution to the growing literature on discrimination from minorities' perspectives.

\section{Economic vs. Psychological effects of discrimination.}

The discrimination phenomenon involves either actions by the perpetrators or effects on target groups (Feagin \& Feagin, 1978). From the targets' perspective, the consequences of discrimination are increasingly being differentiated by social scientists in terms of economic and psychological outcomes of discrimination. Government surveys are often used in empirical studies to analyze the economic effects of discrimination. These surveys provide statistical information on wages disparity and housing segregation of target groups (Feagin \& Eckberg, 1980). Disparities in economic outcomes of minority groups as a consequence of discrimination are different from the economic resources inequality outcomes described by Therborn (2006). The latter pertain to disparities between low-income and high income countries and the segregation between the rich and the poor within these countries.

Other economic impacts of discrimination on target groups include reduced employment and lower remuneration (Frieze, Olson, \& Good, 1990; Tienda, Donato, \& Cordero-Guzman, 1992). The general long-term economic effects of discrimination have been established in conjunction with prolonged unemployment that led to deterioration of skills, learning abilities and motivation (Elsmlie \& Sedo, 1996) and wage differentials that penalized even the second generation minority (Blackaby, Leslie, Murphy, \& O'Leary, 2005). 
In terms of psychological effects, discrimination has been found to have disturbing consequences on individuals despite the solutions generated to curb it. Psychological outcomes are usually examined in relation to satisfaction and well-being measures in the context of the workplace and life in general.

\section{Actual experience vs. Perceived discrimination.}

Theories proposing cognitive appraisals as the key to understanding the effects of stressors support the view that ample focus should be given to perceived discrimination parallel to discriminatory experience (Lazarus \& Folkman, 1984; Sanchez \& Brock, 1996). Dipboye and Collella (2005) argue that perceptions of discrimination or perceiving oneself as a recipient of discriminatory acts is as significant as the actual incidence of discrimination. Cognitive appraisals of an individual's experience help in the assessment of the challenges within the person's environment (Lazarus \& Folkman, 1984). Perceptions propel individuals to respond and make choices on what coping strategy are appropriate to combat discriminatory situations (Chung, 2001). Resulting attitudes and behaviours have been also documented to be affected by perceptions of discrimination (Bell, Harrison, \& McLaughlin, 1997; Fried, Levi, Billings \& Browne, 2001) alongside the actual discriminatory experiences.

Moreover, focus on using individual measures for perceptions of discrimination circumvent the problems of creating objective measures of discrimination (Cassidy, O’Connor, Howe \& Warden, 2005). Perception of discrimination is herein defined as the perception of unfair treatment because of the individual's group membership (Ensher et al., 2001; Sanchez \& Brock, 1996). Cardo (1994) similarly defines perceptions of discrimination as 
representations of individuals' assessment that they are being treated unfairly on the basis of their group membership. Hence, instead of assessing actual discriminatory experiences of target groups, usually measured in terms of grievance complaints and discrimination cases filed in court, more studies are looking into the prevalence of discrimination in terms of target perceptions. Similarly, this study will look into the psychological processes involved in perceiving discrimination from minority perspectives.

\section{Gaps in Discrimination Research}

Discrimination continues to be a relevant issue that deserves attention. Some overt forms of discrimination are believed to have disappeared, but it is feared that more implicit forms have taken their place (Dipboye \& Collela, 2005). Thus, continuing research on more covert forms of discrimination (e.g., institutionalized forms of discrimination usually embedded in organizational structures) should be undertaken to prevent these acts from being institutionalized and considered as a normal way of life. Lack of research related to the investigation of new forms of discrimination as perceived by targets may result in underestimation of the prevalence and detrimental effects on minorities (Deitch et al., 2003).

In addition, increased attention should be accorded to targets' perspectives (e.g. employees of diverse ethnic groups) because they experience the direct consequences of discriminatory acts. The exploration of new targets of discrimination (e.g. immigrants) will also add value to the discrimination research literature since cognitions and behaviours of a relatively under-researched target group will be given the necessary attention it deserves. 
Furthermore, there is a call to go beyond the economic effects of discrimination or the analysis of numerical data (e.g. wage differentials, rates of unemployment or underemployment) to conclude that discrimination exists (Dipboye \& Collela, 2005). More focus on psychological constructs related to the experience or perceptions of discrimination (e.g., antecedents and outcomes) can broaden our understanding of the nature, prevalence, mental and physical impacts of discrimination on target groups. More importantly, there is a continuing need to conduct systematic studies using multiple methodologies (e.g. combination of qualitative and quantitative methods) to determine the prevalence and impact of the continuing presence of discrimination in our society.

This thesis intends to advance psychological theory and integrate research concepts on discrmination. The general aim is to provide a research framework to examine the nature of perceived discrimination (PD) from the perspective of target groups and relate these conceptualizations to possible antecedents and outcomes of perceived discrimination. Finally, it is also the goal of this research to develop an appropriate tool for measuring perceptions of discrimination of target individuals in a specific area (e.g., employment) that is appropriate to the evolving definitions and conceptualizations of discrimination.

\section{Overview of the Chapters}

The current thesis consists of eight chapters and four studies:

Chapter 2 provides a broad review of the discrimination literature on race/ethnicity-related PD and a quantitative summary of studies conducted on race/ethnicity-related perceived discrimination using meta-analytic techniques 
(Study 1). The meta-analysis aims to examine trends in empirical research on PD and its correlates across contexts examined in previous research and to identify gaps in PD research.

Chapter 3 serves as an introductory chapter for the upcoming studies in the succeeding chapters and outlines the theoretical framework proposed for this thesis. Gaps in research identified in the meta-analysis will serve as a starting point for the conceptualization of the framework and the empirical studies.

Chapter 4 presents a qualitative study (Study 2) that investigates the nature of PD as experienced by immigrants. Focus Group Discussions were conducted with selected immigrants in New Zealand. Emerging themes are used for the subsequent conceptualization and design of new measures of perceived discrimination.

Study 3 is described in two parts, across Chapters 5 and 6. Chapter 5 presents a review of the existing PD measurements and the development of three PD scales. The psychometric properties of the PD scales focusing on immigrant employees are examined and evaluated. Chapter 6 then reports the relationship between the new PD scales with outcome variables. Organizational and psychological well-being outcomes are studied as two groups of outcome variables.

Chapter 7 focuses on proximal and distal antecedents of PD as discussed in Chapter 3. The predictive ability of these variables is examined in Study 4 using a large group of mixed employees as survey participants. The sample is composed of immigrant and native-born employees from New Zealand organizations. 
Finally, Chapter 8 revisits the framework for the study of PD, discussing and integrating the major findings. It also notes limitations, identifies areas for future research, and discusses implications for organizations and policy makers. 


\section{Chapter 2}

\section{ANTECEDENTS AND OUTCOMES OF PERCEIVED DISCRIMINATION: A META-ANALYSIS (STUDY 1)}

\section{Introduction}

This chapter reviews previous research on race/ethnicity-related perceptions of discrimination (PD) from the target's perspective and examines PD's relationship with correlates (i.e. variables that have been studied in conjunction with PD as antecedents and/or outcomes) by using meta-analytic techniques. Being delegated to a low status has not been a pleasant experience for minorities and many studies have demonstrated negative outcomes for target groups (Bourguignon, Seron, Yzerbyt \& Herman, 2006). Social comparison theories like Social Identity Theory (Tajfel \& Turner, 1986) and its derivative models are among the main conceptual models used by scholars to explain why minority groups experience negative outcomes in their wellbeing. These theories propose that perceptions of low-status and devaluation in society are a key for understanding effects of PD.

On the other hand, Allport (1954, in Bourguignon et al., 2006) commented that discriminated groups are able to use various strategies to cope with their negative experiences. In support of Allport's statement, an increasing number of studies have shown that stigmatized people are able to utilize strategies, knowingly or instinctively, that help them face discriminatory behaviours from dominant groups (Clark, Anderson, Clark, \& Williams, 1999; Meyer, 2003; Miller \& Kaiser, 2001). This alternative approach of individuals as active agents in the discrimination process has complemented the studies that mainly focused on the negative consequences 
of perceived discrimination on individuals. Despite the varying disparate opinions regarding the nature and impact of $\mathrm{PD}$, the extent to which $\mathrm{PD}$ is associated with various variables has not been systematically investigated. Thus, a research summary is thereby necessary to provide a more systematic analysis of the current impact of PD on target individuals.

To help me make sense of the growing divergence in the discrimination literature, I set several goals to guide me throughout this study. The specific objectives of this study are a) first, to examine the average effect of PD on its correlates, b) secondly, to identify if the effect is homogenous across different constructs and c) finally to identify if the effects are homogenous for each correlate separately (and to explore potential moderators that explain the divergence of findings). The findings are intended to expand the understanding of the relationships PD has with various predictor and outcome variables. Also, the results of this study are aimed to be a valuable source in identifying gaps in research so that these missing areas in the literature will be properly addressed through the proposal of future avenues for studies that could advance the knowledge about race/ethnicity-related perceptions of discrimination.

The introductory section of this chapter discusses the importance of meta-analysis as a research tool, followed by an outline of the different research domains that provided the contexts on race/ethnicity-related perceived discrimination studies leading to the statement of hypotheses.

\section{Meta-analysis as a Research Methodology}

Perceptions of discrimination have been operationalized broadly distinguishing various forms and levels based on different theoretical 
frameworks. Traditionally, narrative reviews are used by researchers to summarize the studies done on a particular topic. Recently, however, qualitative reviews are regarded as less effective in providing explanations of the nature of a phenomenon for several reasons, namely: a) they can only cover a limited number of studies; b) they provide less description about study characteristics and the quality of the methodology used; c) they are unable to reach conclusive statements about differing findings, and most importantly d) they minimize the significance of effect size magnitude only relying on statistical significance (Johnson \& Boynton, 2008).

On the other hand, Lipsey and Wilson (2001) enumerated several advantages of conducting meta-analysis in analyzing a large body of data. First, meta-analysis summarizes studies using a strict and systematic set of procedures that are open for public enquiry. Secondly, weighted averages of the effect sizes are obtained to estimate a single population effect size. Third, relationships of variables that are embedded in the studies can be further scrutinized by meta-analysis and potential moderators can be explored. Lastly, although it is desirable to have a large number of studies to be included in an analysis, a small number of studies can also have meaningful output. Nevertheless, meta-analysis can a large body of data since one can code information as detailed as the researches wants it to be. Henceforth, this methodology is deemed very useful in conducting an in-depth review of large quantities of data.

Thus, instead of conducting a qualitative review of existing studies on PD, a meta-analytic study was deemed a more appropriate tool to achieve this study's major objectives based on the mentioned qualities of the method. It is 
further believed that conducting an in-depth quantitative analysis of the current research domain is a prerequisite for the development of constructs and theory building (Ng, Eby, Sorensen, Feldman, 2005; Reichers \& Schneider, 1990). A quantitative summary provided by meta-analysis will provide an overall picture of the relationships between discrimination and PD and other variables as well as an overview of research done in this area.

The succeeding sections identify different research domains and theoretical models that looked into the investigation of PD and the resulting relationship with various psychological constructs that were considered as antecedents, outcomes or simply a correlate. Secondly, PD has also been studied with demographic variables. Similar to the study of PD in the different domains, theoretical models were also used to assess the importance of these demographic variables in relation to PD.

\section{Research Domains}

Discrimination continues to persist in various areas of life (ILO, 2003) and perceptions of it allow individuals to act on the problem at hand (Dipboye \& Collela, 2005). Knowing to what extent perceptions of discrimination (PD) relate to different individual outcomes is a good gauge of the robustness of the concept of discrimination as a detrimental aspect of an individual's experience. Most studies conducted on the psychological correlates of PD are in the areas of social psychology and sociology while a growing number of studies are found in management literature (Alderfer, Alderfer, Tucker, \& Tucker, 1980; Cox, 1993; Foley, Hang-Yue, \& Wong, 2005; Tajfel \& Turner, 1986). Consequently, race- or ethnicity-related discrimination and the perceptions of it by target groups have been increasingly studied and scholars 
generally follow a few theoretical approaches that are often used in their particular research domains.

The domains were organized in this review as social psychology, personality, health psychology, and organizational psychology. Much of these domains overlap since the conceptual foundations of these perspectives usually refer back to the same theoretical frameworks or new theoretical models are based on similar major theories.

\section{Social Psychology}

Scholars in Social Psychology have attempted to comprehend the phenomenon of discrimination by understanding the basic assumptions of human behaviour. It is believed that central to the individual is the understanding or the clarification of his/her self-concept. Social Identity Theory (SIT) proposes that the self-identity as a component of the self concept is manifested in an individual's qualities on a personal level and as a member of a social group (Tajfel \& Turner, 1986). Originally conceptualized by Tajfel and Turner $(1979 ; 1986)$, SIT proposes that people segregate themselves based on certain criteria which they can readily identify with such as demographic groups (e.g. gender, race, ethnicity) and/or social groups (e.g. religion, affiliations to organization). This process of self-categorization (Turner, Hogg, Oakes, Reicher \& Wetherell, 1987) results in several preferred groups and social categories, but when confronted with conflicting dimensions of social identity, individuals will identify more with similar others that are most salient to them, and this becomes the basis for self-definition.

Since the basis for the self concept is group membership, the individual will make social comparisons with the in-group members. In order to maintain 
a positive self-concept, individuals would stay in a group that gives them a sense of belonging. Negative perceptions about the in-group, on the other hand, will prompt individual strategies to maintain the positive sense of self depending on the boundary permeability of the group to which the individual is attached (Tajfel, 1981).

Social Identity Theory and other derivative models such as the Rejection-Identification Model of Branscombe and colleagues (1999) provide insights into how negative evaluations of the in-group like discrimination (with members of their own category) are positively related to in-group identification. Social Identity Theory (Tajfel \& Turner, 1986) also outlines that group categorization and group comparisons may result in in-group bias and negative attitudes towards out-groups. Tajfel (1978) believes that in cases of discrimination related to race, targets are subjected to unfair practices not because of individual differences (e.g. personal beauty) but because they are part of a race (e.g. being black). He further interjects that individuals usually act foremost as part of a "well-defined and clearly distinct social categories" (page 27). Hence, an individual will be more aware of social situations (e.g. event of discrimination) depending on the individual's level of attachment to the group which is largely influenced by the clarity of his/her awareness that he/she belongs to a group, the quality of his/her membership within the group, and the extent of his/her investment emotionally in the in-group awareness and in-group evaluations (Tajfel, 1978).

Thereby, perceptions of discrimination and prejudice may thrive in the context of salient comparisons between one's in-group and an out-group. Drawing from Sherif (1967, in Tajfel \& Turner, 1986), Tajfel and Turner 
(1986) mentioned that when society is stratified as a function of unequal resources (e.g. wealth, power), realistic conflict arises between groups. Unequal division of scarce resources in turn is associated with higher rates of ethnocentrism and out-group antagonism between the over-privileged and underprivileged groups. Subsequent theorists have argued that awareness that privileged majority groups are prejudiced and discriminatory towards members of one's in-group (minority) results in increased identification with the in-group (Branscombe, Schmitt \& Harvey, 1999). Therefore under particular conditions, a reinforcing cycle is set in motion, in which individuals with higher group identification are more likely to perceive more discrimination and react more negatively to other groups and greater discrimination leads to stronger identification with the group depending on options available to group members.

Conversely, Tajfel acknowledged that aside from cognitive processes, there is a motivational factor that can explain further how identification relates to in-group bias and out-group discrimination (Huddy, 2001). This is the need for a positive social identity or a positive group image (Jost \& Elsbach, 2001). Members of high status group are viewed to develop stronger group identity since this defines them positively relative to other groups. Moreover, high status members are believed to engage in identity protection (van Knippenberg \& Ellemers, 1993). Thus, members of privileged groups tend to respond negatively to high permeable boundaries since this poses a threat to their high status. In support to this, Ellemers et al. (1988) found that high permeability was negatively related to in-group identification for low-status members and 
positively related to increased identification with the group for members of high status groups.

In contrast, for low status groups, Tajfel and Turner (1986) further explained that propensity to engage in intergroup competition may depend on the permeability of boundaries in the social structures. Hence, more permeable boundaries mean higher possibility for individual mobility that may lead to less in-group identification. This means that underprivileged members may opt to leave or disconnect from their group. On the other hand, if individual mobility is restricted, low status members direct their efforts toward strengthening positive in-group identification through social action or social creativity. Findings supported this view such that devalued group members tend to exaggerate positive characteristics of their group to show supremacy over the other group (Mummendey \& Schreiber, 1984; van Knippenberg \& van Oers, 1984). Moreover, other studies found that participants were less likely to participate in collective protest if perceived intergroup permeability is low (Wright, Taylor \& Moghaddam, 1990) while members of a losing hockey team (i.e. low status group in a non-permeability situation) engaged in ingroup bias on qualities unrelated to team performance (Lalonde, 1992).

Nonetheless, SIT has been criticized to have been overly engrossed in intergroup boundaries and have overlooked the importance of the subjective or internal meaning of identities specifically in the political domain (Huddy, 2001). Previous studies reviewed by Huddy (2001) involving minority groups like African Americans in the United States, Arabs in Israel and South Africans reveal that strong ethnic and racial identities may lead to lower nationalized patriotism, intolerance and inter-group antagonism. 
More studies on minority perceptions especially on devalued groups referred to the views presented by SIT to explain how negative perceptions of the in-group like prejudice and discrimination increase identification with that in-group. It is believed that increased identification in negative situations stems from the individuals' need to belong (Branscombe et al., 1999). In general terms, it has been found that threats to the group can increase group cohesion and identification (Turner, Hogg, Turner \& Smith, 1984). Moreover, lack of opportunity for individual mobility and negative perceptions like prejudice and discrimination also result in increased identification among lowstatus individuals (Ellemers, 1993; Jett, Branscombe, Spears \& Smith, 2001). Similarly, Branscombe and colleagues (1999) reviewed various studies done on Jews, women groups, African Americans, Hispanics, lesbians, nonconformist college groups (e.g. punks) that all support that awareness of prejudice is related to increased group identification.

Since individuals are driven by the need to belong, and when low status group members realize that fair treatment from high status group is not possible, the strategy that they use to enhance their positive sense of self and well-being is identifying with the in-group (Branscombe et al., 1999). Accordingly, Branscombe and colleagues (1999) developed the RejectionIdentification Model to show that attributions to negative events like prejudice and discrimination have both positive and negative effects on well-being through identification with one's group. They found that attributions to prejudice are positively associated to minority group identification and their African American participants view racial discrimination as unlawful and they feel that they deserve equal treatment. Participants identify more with minority 
groups and resent the majority group if they view that they are not treated equally in various conditions.

Moreover, other studies have shown that the more individuals are attached to their in-group, the more receptive they are to stigmatization and the easier they make attributions of discrimination (see review of Major, Quinton \& McCoy, 2002) from external factors, especially if evidence of discrimination is present and the group identity is salient (Crocker \& Major, 1989; Friedman \& Davidson, 1999).

Furthermore, Barry and Grilo's (2003) review states that higher PD is more likely to be reported by ethnic minority groups with strong ethnic identification. Moreover, studies that have looked into the correlations of ethnic identity and PD generally show positive relationships (e.g., Evans \& Herr, 1994; Hall \& Carter, 2006; Jefferson \& Caldwell, 2002; Romero \& Roberts, 1998; Scott, 2003; Sellers \& Shelton, 2003). Some studies appear to find negative relationships but these failed to reach statistical significance (e.g., Cassidy et al., 2005; Lee, 2003; Phinney et al., 1998).

Thus, based on various study findings presented in the preceding sections, the general direction of PD's relationship with racial/ethnic identity suggests the following hypothesis:

Hypothesis 2.1: PD is positively associated with racial or ethnic identity

\section{Personality-Related Domain}

Most studies that relate PD with personality-related variables follow the attributional perspective (Phinney et al., 1998). This perspective relies on 
theoretical frameworks that focus more on individual differences like personality-related constructs. As earlier mentioned, the self-concept has been regarded by early scholars as a product of interaction with others and expression of other people's perspective about one's self. As explained by Social Comparison Theory (Festinger, 1954, cited in Verkuyten, 1993) and further elaborated by Symbolic Interaction Theory (Cooley, 1902; Mead, 1934; in Verkuyten, 1993), individuals tend to view themselves as significant others would see them during the process of social comparison.

Related to the above theories, Crocker and Major (1989) have summarized early sociologists' perspectives on "reflected appraisals" or the "looking-glass self" and came to the conclusion that the self-concept is a result of an individual's awareness of other people's views towards him/her and his/her own interpretation of those views. Furthermore, supporters for the Reflected Appraisal Theory (Kinch, 1963; cited in Verkuyten, 1993) found that self-appraisals (how individuals view themselves) and reflected appraisals (individuals' opinion of how other peoples see them) are substantially related to each other. The theory of reflected appraisal has had some criticisms saying that the theory overly emphasizes the importance of feedback of others and the view of the individual as a passive social being (Gecas \& Schwalbe, 1983). However, it adequately explains how individuals, who perceive that their racial/ethnic group are devalued, are more disposed to internalizations of negative feedback from the environment.

Crocker and Major (1989) similarly questioned the assumptions of the theory of reflected appraisal by proposing the Discounting Hypothesis wherein they posit that individuals who are perceiving discrimination may actually be 
acting in a self-serving strategy since they "discount" themselves by attributing the root cause to external factors rather than themselves when faced with failure. Empirical support for this hypothesis has been weak and mostly supported by isolated studies of African Americans who demonstrated higher self-esteem than White Americans (e.g. Kling, Hyde, Showers, \& Buswell, 1999).

Similar to the tenets of reflected appraisal theory, the RejectionIdentification Model (Branscombe et al., 1999) also proposes that perceiving discrimination is similar to being excluded such that it negatively impacts selfesteem. This view has been supported by various studies such that higher PD, as a form of awareness of being treated negatively, usually leads to lower selfesteem (Crocker \& Major, 1989; Lee, 2003, Oppedal, Roysamb \& Sam, 2004; Phinney et al., 1998; Shorey, Cowan, \& Sullivan, 2002). The model further introduced additional concepts to the PD - Self-esteem relationship by suggesting that identification may buffer the effects of PD on self-esteem, such that individuals will tend to identify more with their in-group if being negatively evaluated by an out-group to protect their self-esteem. Specifically, ethnic identity was identified as a moderator that changes the relationship between PD and self-esteem, wherein PD results in higher self-esteem if high ethnic identity buffers the relationship (Romero \& Roberts, 2003).

The rejection-identification model, however, theoretically explains perceived personal discrimination but empirically measures both personal and group perceptions (Bourguignon et al., 2006). Early scholars have pointed out the importance of distinguishing personal PD from group PD (Crosby, 1982). More studies support the view that individuals generally report less personal 
discrimination than group discrimination (Crosby, 1982; Taylor, Wright \& Porter, 1994). In self-esteem studies, when differentiating personal PD and group PD, results showed that personal PD is related to low self-esteem and group PD is associated with higher self-esteem (Barry \& Grilo, 2003). However, stronger identification with the ethnic group lessens the effect of group PD on self-esteem but not on personal PD (Bourguignon et al., 2006). These findings parallel differences in personal and group effects of relative deprivation. In a relative deprivation framework, egoistic or personal deprivation has an effect on well-being while fraternal or group deprivation affects group responses, and identification is an example of a collective response (Hafer \& Olson, 1993; Smith \& Ortiz, 2002; Walker \& Mann, 1987).

Hence, the varying results on the relationship between PD and selfesteem are best analyzed through moderators like individual and group PD measurements. But overall, findings show that perceiving negative feedback like PD is attributed to the self (i.e., ethnic group is devalued), and negatively impacts on self-esteem (Verkuyten, 1993). Thus, the lack of support for the Discounting Hypothesis and the more consistent findings on the direct negative relationship of PD to self-esteem suggests the following hypothesis:

Hypothesis 2.2: PD is negatively related with personality-based constructs like self-esteem.

\section{Health Psychology}

Research on effects of PD on psychological and physical health has typically been conducted in the context of research on socio-cultural adaptation and acculturation of immigrants. Research on acculturation has been using Cognitive Appraisal Theory (Folkman \& Lazarus, 1984) and the 
Stress and Coping Paradigm (Ward, Bochner \& Furnham 2001) in investigating the effects of PD on immigrants' psychological well-being and health. In these theories, the experience and/or cognition of discriminatory events are viewed as stressors that have detrimental effect on individual's health and well-being. Similarly, Berry's (1990) Acculturative Stress Model posits that perceptions of stressors or threat are a person's processing of circumstances that trigger a coping response from the individual with the aim to control the situation and eventually maintain the positive self-concept.

Berry's (1990) Acculturative Stress Model attempts to explain how stressors like perceiving oneself as target or victim of discrimination from majority groups like the host nationals adversely affects the immigrants' acculturation process and eventually results in adverse psychological states. Berry (1990) pointed to psychological indicators such as depression, anxiety, and psychosomatic symptoms as reactions of immigrants to different stressors. If the stressors overpower the individuals' capacity to cope, this leads to negative outcomes. There are mixed results in the studies looking into the most effective coping styles (see Moghaddam, Taylor, Ditto, Jacobs, \& Bianchi, 2002; Noh et al., 1999). Nonetheless, in Europe, higher PD was found to be related to higher psychological stress symptoms (depression, anxiety and psychosomatic stress symptoms) for Russian-speaking immigrants in Finland (Jasinkaja-Lahti \& Liebkind, 2001), higher distress symptoms for immigrants in Norway (Oppedal et al. (2004); higher levels of anxiety and depression for Unite Kingdom-based immigrants (Cassidy et al., 2005), and negative psychological well-being for adult Russian-speaking and Estonian- 
speaking immigrants in Finland (Jasinskaja-Lahti, Liebkind \& Perhoniemi, 2007).

Studies conducted in the United States echo their European counterparts' findings that PD has negative effects on health. Single ethnic group studies found that higher PD leads to greater psychological distress for Latinos (Moradi \& Risco, 2006) and college students of Indian-descent (Lee, 2003). Another widely studied ethnic group in the United States is African Americans who are reported to have conduct problems and depressive symptoms (Brody, Chen, Murry, Simons, Ge, Gibbons et al., 2006); higher levels of depression and perceived stress but not psychological well-being (Sellers, Copelan-Linder, Martin \& Lewis, 2006); higher event-specific and global psychological distress (Sellers \& Shelton, 2003); low emotional wellbeing (being less happy or sad) and low physical well-being (Deitch et al., 2003) when perceiving racism or higher levels of racial discrimination. Multiethnic group studies also found that higher level of PD is related to higher levels of depression/anxiety for first and second generation immigrants (Phinney et al., 1998), higher levels of chronic stress lifetime traumas and depressive symptoms for African-American and non-Hispanic (Taylor \& Turner, 2002) and Black, Latino and Asian Americans (Greene, Way \& Pahl, 2006).

Moreover, the ICSEY multi-country study on immigrant youth from thirteen countries (Australia, Canada, Finland, France, Germany, Israel, New Zealand, Norway, Portugal, Sweden, The Netherlands, United Kingdom and the Unite States) showed that PD was negatively associated with psychological and socio-cultural adaptation. The Vietnamese and Turkish 
youth in Norway reported the highest PD. Previous research thus suggests a consistently negative effect of PD on negative health outcomes:

Hypothesis 2.3: Perceptions of discrimination are positively related to negative health outcomes.

\section{Organizational Psychology}

Interest in measuring the effects of PD on job-related or careeroriented outcomes is usually an offshoot of understanding the intricacies of increasing diversity in the workplace. Most studies look to Social Identity Theory (Tajfel \& Turner, 1986) as a basic tenet for explaining how and why individuals assign themselves to certain categories. One of the most influential theories in organizational research is Relational Demography Theory (Tsui, Egan \& O'Reilly, 1992; Tsui \& O'Reilly, 1989). The theory proposes that individuals compare their own demographics such as race, ethnicity or gender with those of the organization's demographic characteristics to determine similarities and dissimilarities. Subsequently, the extent of variations perceived by the individual in relation to the general composition of the organization will affect his/her job outcomes. Thus, PD is believed to be an immediate outcome of differences in demographic characteristics since perceptions of being different have negative effects on individuals (Riordan, Shaffer \& Stewart, 2005).

The studies linking race or ethnic background with PD have generated consistent results on the effects of PD on organizational outcomes, especially in single race/ethnic studies. Among Hispanic employees, PD has been found to be negatively related to job satisfaction (Foley \& Kidder, 2002; Sanchez \& Brock, 1996), negatively associated with organizational commitment (Sanchez 
\& Brock, 1996), positively related to work tension (Sanchez \& Brock, 1996) and positively related to perceptions of glass ceiling (Foley \& Kidder, 2002). Moreover, for Black American employees, those who perceived higher PD have lower job satisfaction (Deitch et al., 2003) and higher belief that promotional opportunities are based on ascribed characteristics rather than individual capabilities.

Furthermore, the analysis of PD's relationship to organizational variables can be segregated into multiple levels based on the Interactional Model of Cultural Diversity (IMCD) by Cox (1993). The IMCD elaborates on three levels that can be used to understand the effects of PD on different categories, namely (a) individual (b) group-intergroup and (c) organizational levels. This segregation of levels has been used in examining the effects of PD on attitudes and behaviours in the organizational context. The distinction of different levels within the organization has led to studies examining the issue of perceptions of unfair treatment from supervisors/adults, peers/co-workers and from the organizational/labour market structures (Ensher et al., 2001; Jasinskaja-Lahti \& Liebkind, 2001; Tougas \& Veilleux, 1990; Way, 1997). Specifically, Ensher et al. (2001) found that organizational-level PD was a consistently negative predictor of job satisfaction (JS), organizational commitment (OC), and organizational citizenship behaviour (OCB). Supervisor PD was a negative predictor of JS and OC. On the other hand, coworker discrimination was only related to OCB.

In addition to the job outcomes mentioned above, perceptions and experiences of discrimination have been also documented to affect attitudes towards the United States institutional policies like Affirmative Action (AA) 
programs implemented in the workplace (Bell et al., 1997; Fried, et al., 2001). Affirmative Action programs are a set of policies with the goal to increase representation of historically less favoured groups and often include timetables and guidelines to ensure the goals are achieved within the required time frame (Konrad \& Linnehan, 1999). Attitudes towards Affirmative Action policies and its relationship with PD have been of growing interest and increasingly studies in the United States are looking into this direction. Studies have found that higher PD is associated with attitudes that are more positive towards Affirmative Action polices (Fried et al., 2001; Son Hing, Bobocel, \& Zanna 2002). Other organizational based variables like perceptions of equal opportunities in promotional and career advancement have been linked to PD in isolated studies. For instance, lower levels of PD have been found to be related to higher perceptions of procedural justice (Slaughter, Bulger, \& Bachiochi, 2005) and higher perceptions of promotional opportunities (Foley \& Kidder, 2002). However, only job satisfaction and attitudes towards Affirmative Action policies have been studied enough with PD to be included in the analysis. The general direction of PD's relationship to organizational and job-related variables supports the following hypotheses:

Hypothesis 2.4a: PD is negatively related to job satisfaction

Hypothesis 2.4b: PD is positively related to attitudes favouring Affirmative Action (AA) policies

\section{Discrimination and Demographic Variables}

Phinney et al. (2008) maintain that theoretical frameworks can be basically categorized into two approaches, the socio-cultural perspective that puts emphasis on demographics characteristics of individuals and the 
attributional perspective that focuses more on psychological differences among individuals. Social comparison theories like social identity (Tajfel \& Turner, 1979; 1986) and self-categorization theories (Turner et al., 1987) and their derivative models like Relational Demography Theory (Tsui et al., 1992), generally follow the socio-cultural perspective giving more importance to demographic variables such as race/ethnicity, age, gender and socio-economic status (SES) as relevant correlates of PD.

Scholars have initially looked into demographic differences in terms of race, ethnicity, or gender to investigate prevalence of PD. Recently, more demographic variables are currently being used in understanding the varying effect of PD on different outcomes. One of these variables is Socio-Economic Status usually measured as individual or a combination of personal income, occupation and educational attainment (Schittker \& McLeod, 2005). SocioEconomic Status is increasingly being used as an economic indicator of comparative group outcomes between majority and racial/ethnic minority groups, immigrants and native-born and the like. Income disparity is considered as an indicator of economic discrimination (Schittker \& McLeod, 2005) supported by findings that Socio-Economic Status is negatively related to PD (e.g. Taylor \& Turner, 2002).

However, study findings show mixed results such that Socio-Economic Status was found to be positively related to PD (e.g. Gibbons, Gerrard, Cleveland, Wills, \& Brody, 2004), while some results show negative, but insignificant relationships (e.g. Ensher et al., 2001; Evans \& Herr, 1994; Phinney et al., 1993; Romero \& Roberts, 1998). Specifically, it was found that the African American parents' PD was positively related to Socio-Economic 
Status showing that parents who have higher Socio-Economic Status would experience more discrimination since they will be more likely to interact with their White counterparts (Gibbons et al., 2004). Gibbons and colleagues (2004) also found that Socio-Economic Status was negatively related to children's PD but they dismissed this as unreliable because they were not able to observe the same findings at Time 2. It has been pointed out that current studies in Relational Demography Theory research mostly focused on the effects of demographic variables on outcomes (e.g. job satisfaction or organizational commitment) and not on proximal or immediate effects like PD (Riordan et al., 2005). Thus, not much attention has been accorded on this area leading to inconsistent findings in the literature. The mixed results of the effects of Socio-Economic Status as a demographic on PD thereby lead me to propose a research question instead of a hypothesis, thus:

Research Question 2.1: How is Socio-Economic Status related to perceptions of discrimination?

\section{Moderating Effects of Contextual Variables}

There are variables based on the characteristics of the studies included in the meta-analysis that may relate to variations in effect sizes (Frattaroli, 2006). Moderators are essential because they can clarify inconsistencies among study findings (Johnson \& Boynton, 2008). In this research, the immediate impact of study and participant characteristics on the relationship of PD with its correlates was investigated. Study characteristics are composed of study context, types of measurement (frequency scales or non-frequency scales) and levels of measurement (single-level or multi-level measurements like personal or group perceptions of PD). Moreover, participant 
characteristics like age bracket and sample population were also considered as moderators.

\section{Study Characteristics}

There are aspects of the research design such as study characteristics that could influence effect sizes. Previous studies correlate PD with variables pertaining to generalized beliefs that individuals can control outcomes and can cope with different problems that confront them. These individual strategies are usually personality-related but individual reactions can be categorized as situation- or domain-specific such as in school or work environment (Wang \& Richarde, 1988). Similarly, closer inspection of the studies selected for the meta-analysis show that PD was examined in several contexts like school, workplace or community settings. Thus, these different settings may have differential effects on the relationship between PD and its correlates.

Secondly, variations in how PD is measured may produce different results. In terms of types of PD scales, either studies use frequency scales or non-frequency scales (usually interval scales). Perceptions of discrimination have been regarded as a stressor, such that constant exposure to it will lead to negative outcomes. Thus, PD was measured using scales that looked at frequency of discriminatory events to determine the effects of "constant exposure". On the other hand, other studies have used measures that are not time-bound. Hence, PD was measured through scales asking participants to rate how much they perceive discrimination in general. Thereby, PD can be observed in different settings and can be measured using different types of scales. 
The last study characteristic considered as moderator is the level of PD measurement. There is an on-going debate regarding the importance of differentiating personal PD from group PD. For instance, the negative association of PD with self-esteem and the positive association of PD with ethnic identity are well established. However, the relationship of PD (whether positive or negative) has been found to be dependent on how PD was measured, such that personal PD can be negatively related to low self-esteem and group PD is associated with higher self-esteem (Barry \& Grilo, 2003; Bourguignon et al., 2006). Moreover, findings with negative but nonsignificant relationship between $\mathrm{PD}$ and racial identity were obtained in studies that have distinguished personal PD and group PD (e.g., Phinney et al., 1998). Increasing interest is also accorded to hierarchical PD such as perceptions from peers, supervisors or systemic. For instance, a study has shown that supervisor PD and systemic PD are more related to organizational outcomes than co-worker PD (e.g., Ensher et al., 2001). Hence, looking at the study design is important in the in-depth analysis on the nature and effects of PD. The role of study characteristics as moderators will be explored in the current study as the following research question instead of a hypothesis:

Research Question 2.2: How are study characteristics related to the relationship between PD and its correlates?

\section{Participants' Characteristics}

Study variables like the use of special populations (Stock, 1994) can create variations in effect sizes. For instance, meta-analytic studies by Smyth (1998) and Frisina et al. (2004) have shown that psychological health effect sizes for students are larger than for non-students. Frattaroli (2006), however, 
gathered opposite results wherein non-students demonstrated higher psychological health effect sizes than students. Thus, more investigation of setting variables should be given attention in investigating effect sizes. The studies included in the meta-analysis used different participant population like students and non-student samples. The non-student sample could vary from employees to association members or community members that participated in a survey.

Secondly, PD has also been studied across different age groups. For instance, most self-esteem and locus of control studies involve youth participants and were mostly done within the school context. It is primarily because adolescence is considered a critical stage in the formation of identity and self-concept (e.g. Greene, Way, \& Pahl, 2006; Harter, 1999).

In the absence of existing references that could support participant characteristics, specifically age and sample population, as moderators of PD and its relationship with correlates, the role of these variables will be explored in the current study thus presenting the following research question instead of a hypothesis:

Research Question 2.3: How are participant characteristics related to the relationship between PD and its correlates?

\section{Summary of Proposed Hypotheses}

In summary, the literature suggests direct relationships of PD with identity, self-esteem, negative well-being, job satisfaction, AA attitudes and SES, leading to the following hypotheses: 1) PD is positively related to group identification measures like racial/ethnic identity, 2) PD is negatively related to personality-based constructs like self-esteem, 3) PD is positively related to 
negative well-being 4) PD is negatively related to organizational outcomes like job satisfaction and AA attitudes. Finally, a research question on the relationship of PD with SES was explored.

In every domain, some variables may change the direct relationships of PD with its correlates. This shows that despite the relative stable relationship established between PD and its correlates, there may be variables that can affect the relationship. Thus, this study looks into study characteristics and participant characteristics as contextual variables in the analysis. The role of these moderators on the relationship of PD with its correlates will be explored thus the proposal of separate research questions for study characteristics and participant characteristics.

\section{Method}

\section{Article Identification and Selection}

\section{Literature Search}

A computerized bibliographic search was conducted in April 2007 using PSYCINFO using the options 1) from the earliest publication up to 2007 and 2) journal articles only. The keywords that were entered were "perception of discrimination" and "perceptions of discrimination". Moreover, reference lists of existing literature on discrimination were also searched for possible additional empirical studies that could be included in the study. An email was sent to the authors of additional empirical studies found requesting results of their studies.

\section{Selection Criteria}

The criteria for inclusion of the article in the meta-analysis were as follows: (a) It should be an empirical study; (b) It must be reporting at least 
one measure of perceptions of discrimination relating to race, ethnicity or immigrant issues and at least one specific measure of a correlate or an outcome; (c) The article had to examine perceived discrimination at an individual level of analysis thus groups as unit of analysis (e.g. team responses) are to be disregarded; and (d) sufficient data was reported to calculate effect sizes (correlation coefficients).

\section{Studies Selected}

The initial search in PsychINFO resulted in 220 hits and reference lists were likewise scanned for additional articles. However, only 44 journal articles passed the selection criteria. Thus, overall, the meta-analysis includes 44 articles reporting 63 individual studies (21 field studies and 42 schoolbased studies). The total number of participants is $37,790(24,476$ for the field studies and 11,314 for the school-based studies). Please see Table 2.1 for the detailed descriptions of the studies. Most of the studies were conducted in the United States $(82 \%)$.

\section{Coding of Variables}

In meta-analytic procedures, it is common to combine variables that are conceptually similar in the coding process (Griffeth, Hom, \& Garetner, 2000; Ng et al., 2005; Viswesvaran, Sanchez, \& Ficher, 1999). Thus, we categorized related variables into a single category as outlined in the succeeding paragraphs. The general guideline used in the decision to create a category of variable is that the number of studies for that category should be greater than 3 to attain a reasonable level for meaningful analyses. 
Table 2.1

Overview of Studies on the Correlates of Perceptions of Discrimination

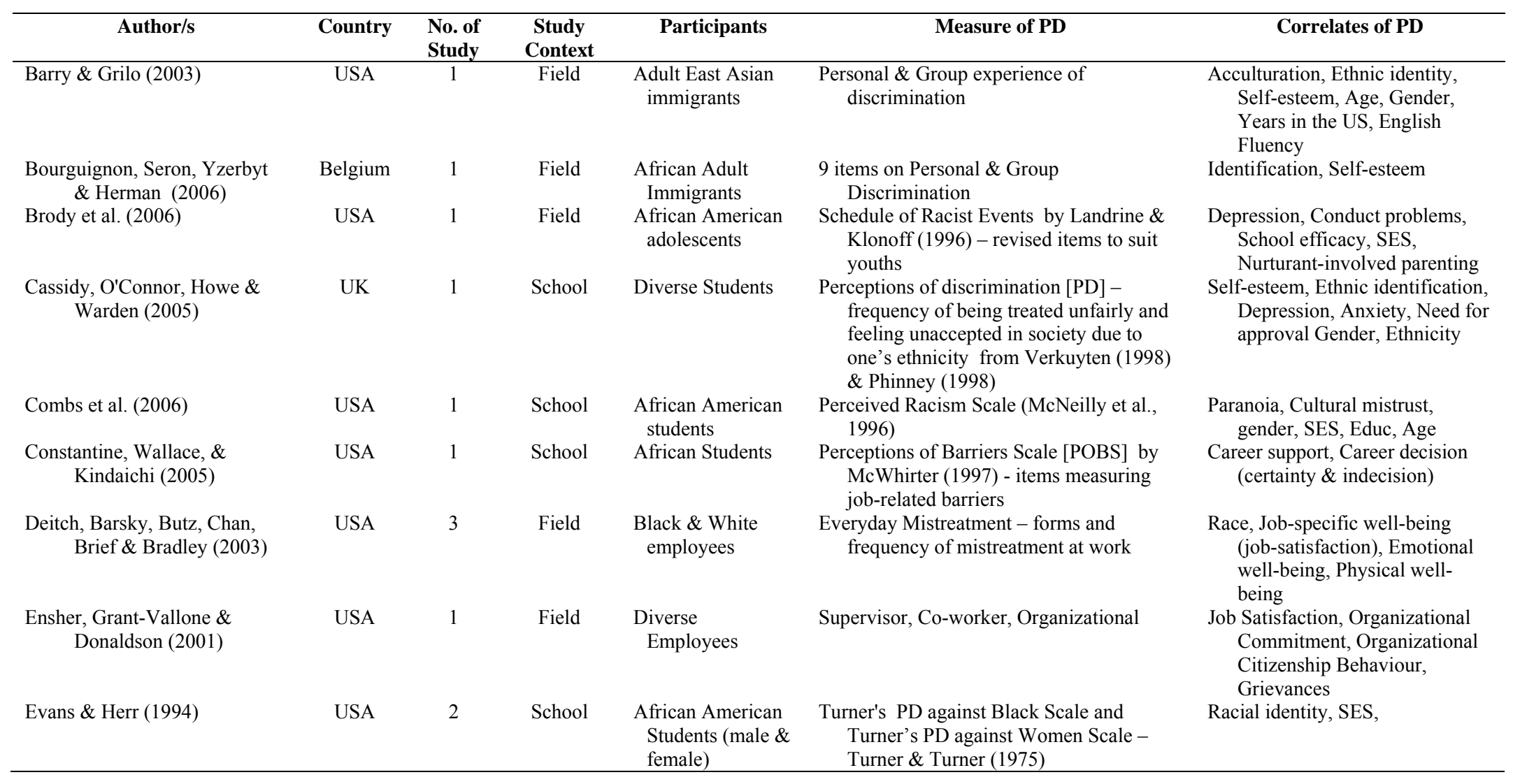


Table 2.1 (continued)

\begin{tabular}{|c|c|c|c|c|c|c|}
\hline Author/s & Country & $\begin{array}{l}\text { No. of } \\
\text { Study }\end{array}$ & $\begin{array}{c}\text { Study } \\
\text { Context }\end{array}$ & Participants & Measure of PD & Correlates of PD \\
\hline $\begin{array}{l}\text { Flores, Navarro, Smith \& } \\
\text { Ploszaj (2006) }\end{array}$ & USA & 1 & School & $\begin{array}{l}\text { Mexican Male } \\
\text { Students }\end{array}$ & $\begin{array}{l}\text { POBS by McWhirter (1997) that } \\
\text { assesses occupational \& } \\
\text { educational barriers }\end{array}$ & $\begin{array}{l}\text { Parents' career choice } \\
\text { nontraditionality, } \\
\text { Nontraditional career self- } \\
\text { efficacy, Nontraditional career } \\
\text { interests, Career choice goal } \\
\text { nontraditionality, Parental } \\
\text { support }\end{array}$ \\
\hline Foley \& Kidder (2002) & USA & 1 & Field & $\begin{array}{l}\text { Hispanics in legal } \\
\text { profession }\end{array}$ & $\begin{array}{l}\text { Perceived Ethnic Discrimination - a } \\
\text { modified version of PD scale by } \\
\text { Sanchez \& Brock (1996) }\end{array}$ & $\begin{array}{l}\text { Satisfaction w/ legal profession, } \\
\text { Procedural justice, Perceived } \\
\text { career prospects, Gender }\end{array}$ \\
\hline Foley, Kidder \& Powell (2002) & USA & 1 & Field & $\begin{array}{l}\text { Hispanics in legal } \\
\text { profession }\end{array}$ & $\begin{array}{l}\text { Perceived Discrimination against } \\
\text { Hispanic Scale (Sanchez \& } \\
\text { Brock, 1996) }\end{array}$ & $\begin{array}{l}\text { Glass ceiling, Distributive justice, } \\
\text { Career prospects }\end{array}$ \\
\hline Foster, Sloto \& Ruby (2006) & USA & 1 & School & Diverse Students & $\begin{array}{l}\text { Personal Discrimination by Foster \& } \\
\quad \text { Matheson (1995) }\end{array}$ & $\begin{array}{l}\text { Collective action, Self-esteem, } \\
\text { Intergroup Anxiety }\end{array}$ \\
\hline $\begin{array}{l}\text { Fried, Levi, Billings \& Browne } \\
\text { (2001) }\end{array}$ & USA & 2 & School & $\begin{array}{l}\text { Employed African } \\
\text { Students }\end{array}$ & $\begin{array}{l}\text { Experience of racial discrimination } \\
\text { in the workplace }\end{array}$ & $\begin{array}{l}\text { Ideology, Affirmative action [AA] } \\
\text { attitude }\end{array}$ \\
\hline $\begin{array}{l}\text { Gibbons, Gerrard \& Cleveland } \\
\text { (2004) }\end{array}$ & USA & 2 & Field & $\begin{array}{l}\text { African Americans } \\
\text { - parents \& child }\end{array}$ & $\begin{array}{l}\text { Perceived discrimination for targets } \\
\text { \& parents using the Schedule of } \\
\text { Racist Events (Landrine \& } \\
\text { Klonoff, 1996) }\end{array}$ & $\begin{array}{l}\text { Distress, Substance use, Image, } \\
\text { Vulnerability, Friends }\end{array}$ \\
\hline Greene, Way \& Pahl (2006) & USA & 1 & School & Diverse Students & $\begin{array}{l}\text { Ethnic and Racial Discrimination by } \\
\text { Way (1997) - PD from adults \& } \\
\text { PD from peers }\end{array}$ & $\begin{array}{c}\text { Ethnic identity, Self-esteem, } \\
\text { depressive symptoms }\end{array}$ \\
\hline Hall \& Carter (2006) & USA & 1 & Field & $\begin{array}{l}\text { Afro-Caribbean } \\
\text { (Jamaican) }\end{array}$ & $\begin{array}{l}\text { Section } 1 \text { of Perceived Racism Scale } \\
\text { (McNeilly et al., 1996) - for the } \\
\text { year, lifetime }\end{array}$ & Racial identity, Ethnic identity \\
\hline
\end{tabular}


Table 2.1 (continued)

\begin{tabular}{|c|c|c|c|c|c|c|}
\hline Author/s & Country & $\begin{array}{l}\text { No. of } \\
\text { Study }\end{array}$ & $\begin{array}{c}\text { Study } \\
\text { Context }\end{array}$ & Participants & Measure of PD & Correlates of PD \\
\hline $\begin{array}{l}\text { Jasinskaja-Lahti \& Liebkind } \\
\text { (2001) }\end{array}$ & Finland & 2 & School & $\begin{array}{l}\text { Russian-speaking } \\
\text { immigrant } \\
\text { adolescents (male } \\
\& \text { female) }\end{array}$ & $\begin{array}{l}9 \text { items - frequency of being } \\
\text { negatively treated by teachers } \\
\text { and peers, being teased or } \\
\text { threatened, feeling of being } \\
\text { unaccepted }\end{array}$ & $\begin{array}{l}\text { Psychological adjustment } \\
\text { (depression, anxiety, } \\
\text { psychosomatic stress } \\
\text { symptoms), Self-esteem, } \\
\text { Parental support, Traditional } \\
\text { family values, Finnish contact }\end{array}$ \\
\hline $\begin{array}{c}\text { Jasinskaja-Lahti, Liekind \& } \\
\text { Perhoniemi (2007) }\end{array}$ & Finland & 1 & Field & $\begin{array}{l}\text { Russian \& } \\
\text { Estonian } \\
\text { speaking } \\
\text { Immigrants }\end{array}$ & $\begin{array}{l}4 \text { items: applying for job, career } \\
\text { advancement, dismissal from } \\
\text { work, racial offense or } \\
\text { harassment from work }\end{array}$ & $\begin{array}{l}\text { SES, Self-rated general health, } \\
\text { psychological well-being, } \\
\text { work-specific group-level } \\
\text { control belief }\end{array}$ \\
\hline Jefferson \& Caldwell (2002) & USA & 1 & School & $\begin{array}{l}\text { African American } \\
\text { Students }\end{array}$ & $\begin{array}{l}\text { Discriminatory Scenarios } \\
\text { Questionnaire (DSQ) }\end{array}$ & Racial identity, \\
\hline Konrad \& Spitz (2003) & USA & 2 & Field & $\begin{array}{l}\text { Diverse College } \\
\text { Faculty - } \\
\text { Sociologists \& } \\
\text { Business } \\
\text { Academics }\end{array}$ & $\begin{array}{l}\text { Overt racism (Klugel \& Smith, } \\
\text { 1983), Belief in labor market } \\
\text { discrimination (Tougas \& } \\
\text { Veilleux, 1990), Perceived } \\
\text { experience of discrimination }\end{array}$ & $\begin{array}{l}\text { Attitudes toward AA, Reciprocal } \\
\text { racism, Traditional attitudes } \\
\text { towards women, Race, Gender }\end{array}$ \\
\hline Lease (2006) & USA & 1 & School & $\begin{array}{l}\text { African American } \\
\text { Students }\end{array}$ & POBS by McWhirter et al. (1998) & $\begin{array}{l}\text { Racial identity, Occupational self- } \\
\text { efficacy, SES, occupational } \\
\text { interest }\end{array}$ \\
\hline Lee (2003) & USA & 1 & School & $\begin{array}{l}\text { Asian Students of } \\
\text { Indian descent }\end{array}$ & $\begin{array}{l}\text { Personal Ethnic Discrimination by } \\
\text { Finch }(2000)\end{array}$ & $\begin{array}{l}\text { Depression, psychological } \\
\text { distress, Self-esteem, Ethnic } \\
\text { Identity }\end{array}$ \\
\hline $\begin{array}{l}\text { Levin, Sinclair, Veniegas \& } \\
\text { Taylor (2002) }\end{array}$ & USA & 3 & School & $\begin{array}{l}\text { Female Students - } \\
\text { African, Latino, } \\
\text { Caucasian }\end{array}$ & $\begin{array}{l}\text { General discrimination - Personal } \\
\text { and Group }\end{array}$ & Ethnicity, Gender \\
\hline $\begin{array}{l}\text { Malcarne, Chavira, Fernandez } \\
\text { \& Liu (2006) }\end{array}$ & USA & 7 & School & $\begin{array}{l}\text { Students - } \\
\text { Diverse, African, } \\
\text { Caucasian, } \\
\text { Filipino, } \\
\text { Mexican }\end{array}$ & $\begin{array}{l}\text { PD a subscale of Scale of Ethnic } \\
\text { Experience (SEE) }\end{array}$ & $\begin{array}{l}\text { Ethnic identity, Social affiliation, } \\
\text { Mainstream comfort }\end{array}$ \\
\hline
\end{tabular}


Table 2.1 (continued)

\begin{tabular}{|c|c|c|c|c|c|c|}
\hline Author/s & Country & $\begin{array}{l}\text { No. of } \\
\text { Study }\end{array}$ & $\begin{array}{c}\text { Study } \\
\text { Context }\end{array}$ & Participants & Measure of PD & Correlates of PD \\
\hline Moradi \& Risco (2006) & USA & 1 & Field & Latino & $\begin{array}{l}\text { Perceived Racism Scale for Latinos } \\
\text { [PRSL] by Collado-Proctor } \\
\text { (1999) - measuring PD across } \\
\text { settings like employment, } \\
\text { academic, public }\end{array}$ & $\begin{array}{l}\text { Psychological distress, Self- } \\
\text { esteem, personal } \\
\text { control/environmental } \\
\text { mastery, Latino and US } \\
\text { acculturation }\end{array}$ \\
\hline $\begin{array}{l}\text { Oppedal, Roysamb \& Sam } \\
\text { (2004) }\end{array}$ & Norway & 1 & School & Diverse Students & $\begin{array}{l}\text { Perceived discrimination in class - } \\
\text { from teachers or peers }\end{array}$ & $\begin{array}{l}\text { Distress, Self-esteem, Social } \\
\text { support, Ethnic Identity, host } \\
\text { and ethnic culture competence }\end{array}$ \\
\hline $\begin{array}{l}\text { Phinney, Madden \& Santos } \\
\text { (1998) }\end{array}$ & USA & 1 & School & $\begin{array}{l}\text { Adolescent } \\
\text { Student } \\
\text { Immigrants }\end{array}$ & $\begin{array}{l}\text { Perceived discrimination - } \\
\text { frequency of being treated } \\
\text { unfairly by peers, teachers and } \\
\text { adults }\end{array}$ & $\begin{array}{l}\text { Self-esteem, Mastery, } \\
\text { Depression/Anxiety, } \\
\text { Intergroup competence, Ethnic } \\
\text { Identity, SES, Gender }\end{array}$ \\
\hline Rollins \& Valdez (2006) & USA & 1 & School & $\begin{array}{l}\text { African American } \\
\text { Students }\end{array}$ & $\begin{array}{l}\text { Racism \& Life Experience Scale } \\
\text { (RaLES) - personal \& group by } \\
\text { Harrell (1997) }\end{array}$ & $\begin{array}{l}\text { SES, Gender, Career decision- } \\
\text { making self-efficacy, Career } \\
\text { task self-efficacy }\end{array}$ \\
\hline Romero \& Roberts (1998) & USA & 1 & School & Diverse Students & $\begin{array}{l}2 \text { items PD - amount of PD against } \\
\text { ethnic background and amount } \\
\text { of PD actually experienced }\end{array}$ & Ethnic identity, SES, Gender, \\
\hline Romero \& Roberts (2003b) & USA & 1 & Field & $\begin{array}{l}\text { Mexican-descent } \\
\text { Students }\end{array}$ & $\begin{array}{l}\text { Sub-scale of a socio-cultural stress } \\
\text { scale (Romero Roberts, 2003) }\end{array}$ & SES, Self-esteem, Ethnic Identity \\
\hline Sanchez \& Brock (1996) & USA & 1 & Field & $\begin{array}{l}\text { Hispanic } \\
\text { Employees }\end{array}$ & $\begin{array}{l}10 \text { items from acculturative stressors } \\
\text { and perceived discrimination of } \\
\text { Mena, Padilla \& Maldonado } \\
\text { (1987) }\end{array}$ & $\begin{array}{l}\text { Job satisfaction, organizational } \\
\text { commitment, Work Tension } \\
\text { Role conflict, Role ambiguity. }\end{array}$ \\
\hline Scott (2003) & USA & 1 & School & $\begin{array}{l}\text { African Americans } \\
\text { Students }\end{array}$ & $\begin{array}{l}\text { Daily Life Experiences (DLE-R) } \\
\text { from RaLES (Harrell, 1997) }\end{array}$ & $\begin{array}{l}\text { Racial identity, Racial } \\
\text { socialization, Distress, Coping }\end{array}$ \\
\hline Scott \& House (2005) & USA & 1 & School & $\begin{array}{l}\text { African Americans } \\
\text { Students }\end{array}$ & $\begin{array}{l}\text { Daily Life Experiences Scale (DLE- } \\
\text { F) }\end{array}$ & $\begin{array}{l}\text { Distress, Perceived control, } \\
\quad \text { Coping }\end{array}$ \\
\hline
\end{tabular}


Table 2.1 (continued)

\begin{tabular}{|c|c|c|c|c|c|c|}
\hline Author/s & Country & $\begin{array}{l}\text { No. of } \\
\text { Study }\end{array}$ & $\begin{array}{c}\text { Study } \\
\text { Context }\end{array}$ & Participants & Measure of PD & Correlates of PD \\
\hline Sellers \& Shelton (2003) & USA & 1 & School & $\begin{array}{l}\text { African Americans } \\
\text { Students }\end{array}$ & $\begin{array}{l}\text { Daily Life Experiences [DLE]by Harrell } \\
\text { (1994) }\end{array}$ & $\begin{array}{l}\text { Black Identity, Depression, Stress, } \\
\text { Anxiety, Psychological distress }\end{array}$ \\
\hline $\begin{array}{l}\text { Sellers, Copeland-Linder, } \\
\text { Martin \& Lewis (2006) }\end{array}$ & USA & 1 & School & $\begin{array}{l}\text { African American } \\
\text { Students }\end{array}$ & $\begin{array}{l}\text { Daily Life Experiences [DLE] by Harrell } \\
\text { (1994) }\end{array}$ & $\begin{array}{l}\text { Depression, Well-being, Perceived } \\
\text { stress, Self-esteem }\end{array}$ \\
\hline $\begin{array}{l}\text { Shorey, Cowan \& Sullivan } \\
\text { (2002) }\end{array}$ & USA & 2 & School & $\begin{array}{l}\text { Students - Anglos } \\
\text { \& Hispanics }\end{array}$ & $\begin{array}{l}\text { Perceived Personal and Group } \\
\text { Discrimination - items from } \\
\text { Kobrynowicz \& Branscombe (1997) }\end{array}$ & $\begin{array}{l}\text { Personal self-esteem, Interpersonal } \\
\text { control, individualism, } \\
\text { collectivism, social dominance } \\
\text { orientation }\end{array}$ \\
\hline $\begin{array}{l}\text { Son Hing, Bobocel \& Zanna } \\
\text { (2002) }\end{array}$ & Canada & 3 & School & $\begin{array}{l}\text { Diverse students } \\
\text { (73\% White) }\end{array}$ & $\begin{array}{l}\text { Workplace Discrimination Scale - } \\
\text { measure PD at individual and } \\
\text { systemic level (Bobocel et al., 1998, } \\
\text { Son Hing, 1997) }\end{array}$ & Attitudes towards $A A$ \\
\hline $\begin{array}{l}\text { Spencer-Rodgers \& Collins } \\
\quad(2006)\end{array}$ & USA & 1 & School & Latino Students & $\begin{array}{l}\text { Racial Ethnic Group Disadvantage } \\
\text { Scale (Spencer-Rogers \& Collins, } \\
\text { 2001) }\end{array}$ & $\begin{array}{l}\text { Self-esteem, Negative public regard, } \\
\text { Racial centrality, Group } \\
\text { attachment, Private regard }\end{array}$ \\
\hline Taylor \& Turner (2002) & USA & 1 & School & $\begin{array}{l}\text { Black \& White } \\
\text { Students }\end{array}$ & $\begin{array}{l}\text { Major events (lifetime) and day-to-day } \\
\text { experiences by Williams et al. } \\
\text { (1997) }\end{array}$ & $\begin{array}{l}\text { Stress, Depressive symptoms, } \\
\text { Ethnicity, Gender, SES }\end{array}$ \\
\hline Verkuyten \& Brug (2002) & Netherlands & 2 & School & $\begin{array}{l}\text { Students - Dutch } \\
\text { \& Surinamese }\end{array}$ & $\begin{array}{l}\text { Perceived discrimination by Ruggiero \& } \\
\text { Taylor (1995) }\end{array}$ & Ethnic identity, Self-esteem \\
\hline Watts \& Carter (1991) & USA & 1 & Field & $\begin{array}{l}\text { African } \\
\text { government } \\
\text { employees }\end{array}$ & Personal Discrimination Scale & $\begin{array}{l}\text { Racial identity, institutional racism, } \\
\text { racial climate }\end{array}$ \\
\hline Zlobina et al. (2006) & Spain & 1 & Field & Adult immigrants & $\begin{array}{l}6 \text { items on PD-frequency due to ethnic } \\
\text { background }\end{array}$ & Socio-cultural difficulty \\
\hline
\end{tabular}




\section{Perceptions of Discrimination}

The measurement of perceptions of discrimination has been approached from different angles. There were single level measurements like race PD, ethnic PD or just plain PD. Further, multi-level approaches involved measurements of PD in terms of personal and group; co-worker, supervisor \& systemic PD; peers and adults, and the like. The third measurement approach was based on frequency wherein PD was measured in terms of daily experiences or major life incidences. Indirect reference to PD such as perceived career barriers was also coded as PD. The different operationalizations of PD were combined in the meta-analysis.

\section{Antecedents/Outcomes of PD}

\section{Identity-related constructs.}

There were a sizeable number of studies looking into racial identity, some more specific like Black identity, racial centrality, ethnic identity, group identification and group identity. All effect sizes of studies using measures of identity were combined. This category was coded as "racial/ethnic identity".

\section{Self-esteem related constructs.}

Constructs that pertains to differences in personality characteristics were also associated with PD. Thus, variables included in this category were personal and global self-esteem, mastery, personal control/environmental mastery, school self-efficacy, nontraditional career self-efficacy, occupational self-efficacy, perceived control, group-level control belief and interpersonal control self-efficacy. All effect sizes of studies related to 
the above-mentioned personality constructs were combined. It was deemed appropriate to combine self-esteem and efficacy-related constructs since studies have shown moderate $r=.49$ (Phinney et al., 1998) to moderately high correlations $r=.61$ (Moradi \&Risco, 2006). This category was coded as "self-esteem" since the majority $(43 \%)$ of the studies is self-esteem related. $^{2}$

\section{Psychological and physical well-being measures.}

Perception of discrimination was found to be correlated with health outcomes in several empirical studies. Sometimes health outcomes are termed in general terms such as psychological adjustment, self-rated general health, psychological well-being, psychological distress, emotional well-being and physical well-being. In some studies, health outcomes are more specific like depression, depressive symptoms, distress, anxiety, intergroup anxiety, paranoia, psychosomatic stress symptoms, perceived stress and coping. Since most of the variables coded were negative indicators of psychological and physical well-being, this group of variables was categorized as "negative well-being" in the subsequent analysis.

\section{Organizational-related constructs.}

There were several career and job-related variables measured in the individual studies that were correlated with PD. Variables included job satisfaction, organizational commitment, organizational citizenship behavior, grievances, work tension, justice perceptions, role conflict and role ambiguity, career support, career decision making (certainty \& indecision), job pursuit intention or career interests/choice/prospects

\footnotetext{
${ }^{2}$ Locus of control-related and efficacy-related construct comprise $33 \%$ and $24 \%$ of the total cases respectively.
} 
(traditional and non-traditional). Affirmative action (AA) attitude towards workplace policies and justice perceptions were also included in a number of studies. Although it was desirable to include all outcomes that were correlated with discrimination it was only job satisfaction (6 studies) and affirmative action ( 8 studies) attitudes that met the minimum number of studies to make a meaningful statistical comparison. Thus, separate categories were created for "job satisfaction" and "AA attitudes".

\section{Socio-economic status (SES).}

Socio-economic Status was usually used as control variables or direct correlate of PD. The studies included in this meta-analysis measured SES as a function of income or salary, level of education (from targets or from parents of targets in case of student participants) and occupation. Different operationalizations of SES were combined in the meta-analysis. The category was then named as "SES".

\section{Moderator Variables.}

For Study Characteristics, Types of measurement pertain to how PD was measured, either in form of frequency counting (counting instances of PD or discrimination experiences) or non-frequency (items in the scale measure general perceptions usually through interval scales). Levels of measurement refers to presence or absence of hierarchical perceptions in the PD measure such as single level (absence of hierarchical perceptions) and multi-level (presence of comparative levels like group vs personal, peer vs superior, lateral vs systemic, etc.). Context of study were coded in terms of school setting (data gathered in schools) and field settings (either work context or public areas). Not all studies conducted in 
school settings have students as participants. A school-based study does not necessarily involve adolescents or student participants since the sample maybe a mix of students and young adults (e.g. Barry \& Grilo, 2003) or non-students like university professors and school administrative staff members (e.g. Konrad \& Spitz, 2003).

As for Participant Characteristics, age brackets like adolescents (20 years old $\&$ below) and adults ( 21 yrs old $\&$ above) were obtained through assigning each reported mean age to a particular bracket. Lastly, sample population were coded as students (reported as college or university students) or non-students (heterogeneous groups such as employees, members of lawyers associations or participants in household/public surveys).

In the statistical analysis, the coding of the five moderator variables are as follows: a) type of scales (coded as frequency=1, non-frequency=2); b) levels of measurement (coded as single-level=1, multi-level=2); c) study context (coded as field studies=1, school-based studies=2); d) age bracket ( coded as adolescents $=1$, adults $=2$ ); and e) sample population (coded as non-students $=1$, students $=2$ ).

\section{Computation of Effect Sizes and Moderator Analysis}

A fixed effect size was deemed an appropriate approach in determining the significance of the overall mean effect size, since this is used if there are a relatively small number of studies to be considered (Hedges, 1994). The effect sizes used in this study were the zero-order correlations presented in the studies. For studies that reported multiple effect sizes for specific categories, the effect sizes were averaged. On the 
other hand, if one study reports different outcome variables (e.g. job satisfaction and organizational commitment), the effect sizes were included in separate analyses. Hence, effect sizes were coded into one of the six correlates of $\mathrm{PD}$, namely, racial/ethnic identity, self-esteem, negative well-being, Affirmative Action attitudes, Socio-economic Status, and job satisfaction. The resulting effect sizes were analyzed separately.

The correlations were converted to z-scores using Fisher's transformation taking into consideration that correlations have a problematic standard error formula, and the standard error will be needed later on for computation of the inverse variance weight, Thus, the weighted mean of the transformed effect sizes across each category of variables was computed with greater weight given to larger samples. The weighted mean effect size (weighted by $N-3$ ) was computed (Rosenthal, 1991). Homogeneity statistics (Q) were computed after calculating the average effect sizes across the studies included. This was done to ascertain whether the variability associated with the average effect size was small enough to argue that the effect sizes were homogeneous across studies. If rejected, moderator analyses were conducted.

\section{Results}

\section{Descriptives}

All main effects (see Table 2.2) were significant and in the expected direction. Estimation of whether the effects sizes are small, medium or large is based on standards set forth by Cohen (1992). Small and positive relationships were found for a) racial/ethnic identity (supporting Hypothesis 2.1), b) negative well-being (supporting 
Hypothesis 2.3) and c) AA attitudes (supporting Hypothesis 2.4b). Negative relationship were found with three correlates, namely a) a small effect size for self-esteem (supporting Hypothesis 2.2), a large negative effect size for job satisfaction (supporting Hypothesis 2.4a); and c) a small effect size for SES. Thus, the findings show that the higher the level of PD, the more the participants identify with their racial/ethnic group, the more their psychological and physical health suffer, and the more favourable their attitudes towards affirmative action policies. Conversely, results also suggest that the higher the $\mathrm{PD}$ the level of job satisfaction decreases, and the lower the self-esteem. In response to the Research Question (RQ) 2.1, higher PD is associated with lower SES.

\section{Moderator Analyses}

All Q statistics values were significant (see Table 2.2) for all main effect sizes indicating that there is a need to test for moderators (Hedges \& Olkin, 1985). Significant Q-statistics indicated heterogeneity in the effect sizes suggesting that the weighted effect sizes were not estimating a similar population mean. We used analysis of variance (Hedges, 1994) to test the two sets of moderators (in total five moderators) for each category of PD correlates.

\section{Race/Ethnic identity.}

Significant results were obtained for all proposed moderators. The effect sizes are negative for studies that used scales with multi-level measurement, field settings, among adult and non-student samples. Thus, higher PD is associated with lower racial/ethnic identification in multilevel PD studies [.26], conducted in the field settings [-.10], among adults 
Table 2.2

Meta-analytic Relationships between PD and Correlates

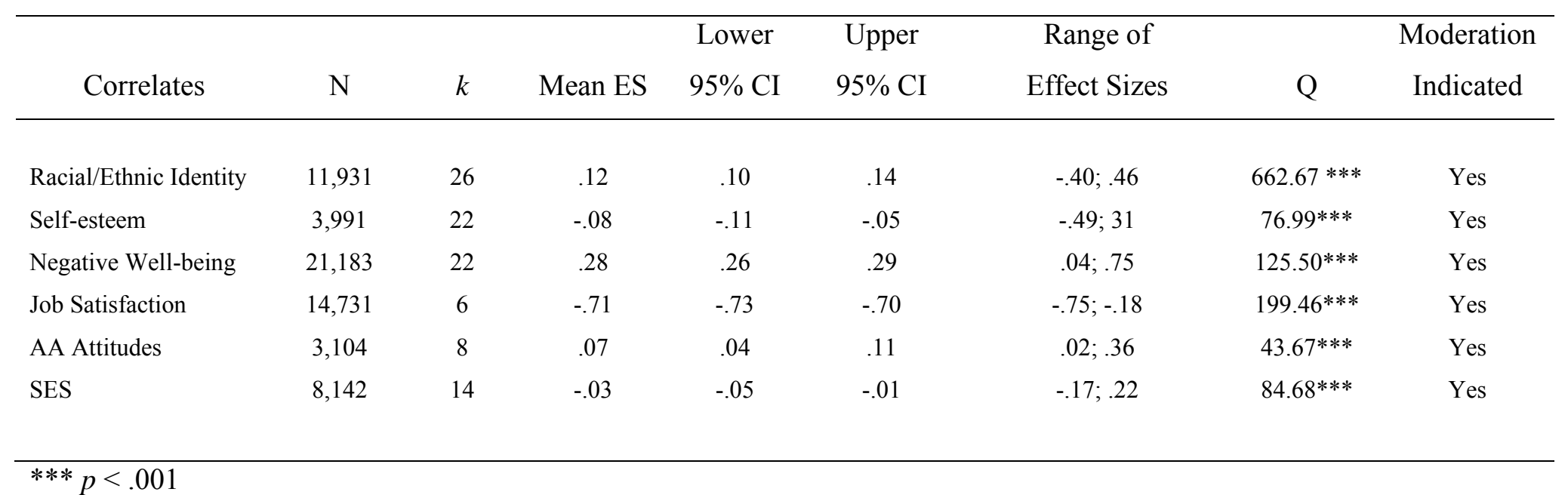

Notes: $P D=$ perceptions of discrimination; $S E S=$ socio-economic status; $A A=$ Affirmative Action; $N=$ cumulative sample size $; k=$ number of studies; ES = effect size CI = confidence interval, $Q=Q$ statistics 
[-.07] and non-students [-.07]. On the other hand, higher PD is associated with higher racial/ethnic identity in studies using single level measurements [.16], non-frequency scales [.16], among student [.20] and adolescent samples [.22].

\section{Self-Esteem.}

The moderation analysis for Self-Esteem did not reach significant levels. Since the overall Q statistic was significant in the initial step indicating heterogeneity, the results in the individual tests indicate that none of the five selected variables were moderating the PD-self-esteem relationship.

\section{Negative well-being.}

Three moderators were found to be significant in explaining variations in PD's relationship with negative well-being namely study context, age bracket and sample population. The significant mean effect sizes were all positive indicating that higher $\mathrm{PD}$ is associated with more negative health indicators, but these effects are stronger in non-academe settings [.28], among adults [.28] and non-student populations [.28].

\section{Job Satisfaction.}

The moderation analysis for study context, age bracket and sample population could not be conducted since all six studies included in the metaanalysis on job satisfaction were field based, adults and with non-student population. For the remaining moderator variables (scale type and levels of measurement), the moderation analysis did not reach significant levels. Since the Q statistics was significant in the initial step indicating heterogeneity, the results in the individual test indicate that none of the three moderators were significant to the PD-job satisfaction relationship. 


\section{Affirmative Action.}

Significant results were obtained for four moderators, namely, levels of measurement, study context, age bracket, and sample population. The results indicate that higher PD is associated with more favourable attitudes towards AA policies especially if multi-level scale measurements are used [.29]. The effects are also stronger if the study is conducted in school contexts [.25] and involved adolescents [.29] or student samples [.25]. Moderation analysis for type of scale was not conducted since all eight studies on AA attitudes in the meta-analysis used general scales or non-frequency scales as measures of PD.

\section{Socio-Economic Status.}

Three significant results were obtained, namely, study context, age bracket and sample population (see Table 2.8). The effect sizes were negative when the studies were school-based [-.05], with adolescents [-.07] and student samples [-.05]. In contrast, higher PD is related with higher SES among adult participants. The relationship was close to zero in field settings and with nonstudents.

Overall, the moderator analysis obtained fifteen significant results (summarized in Table 2.3). The moderators changed the expected direction of PD's relationship with Racial/Ethnic Identity depending on the levels of measurement, study context, age bracket and sample population. Similarly, the moderators also changed the direction of the negative relationship of PD with Socio-Economic Status depending on the study context, age bracket and sample population of the studies. 
Table 2.3

\section{Summary of Significant Moderators}

\begin{tabular}{|c|c|c|c|c|c|c|}
\hline Moderators & $\begin{array}{c}\mathrm{R} / \mathrm{E} \\
\text { Identity }\end{array}$ & $\begin{array}{c}\text { Self- } \\
\text { Esteem }\end{array}$ & $\begin{array}{c}\text { Negative } \\
\text { WB }\end{array}$ & JS & $\begin{array}{c}\text { AA } \\
\text { attitudes }\end{array}$ & SES \\
\hline \multicolumn{7}{|c|}{ STUDY CHARACTERISTICS } \\
\hline Scale Type & Yes & ns & ns & ns & N/A & ns \\
\hline Frequency & positive & & & & & \\
\hline General & positive & & & & & \\
\hline Levels of Measurement & Yes & ns & ns & ns & Yes & ns \\
\hline Single-level & positive & & & & positive & \\
\hline Multi-level & negative & & & & positive & \\
\hline Study Context & Yes & ns & Yes & N/A & Yes & Yes \\
\hline Field & negative & & positive & & positive & zero \\
\hline School & positive & & positive & & positive & negative \\
\hline \multicolumn{7}{|c|}{ PARTICIPANT CHARACTERISTICS } \\
\hline Age Bracket & Yes & ns & Yes & N/A & Yes & Yes \\
\hline Adolescents & positive & & positive & & positive & negative \\
\hline Adults & negative & & positive & & positive & positive \\
\hline Sample Population & Yes & ns & Yes & N/A & Yes & Yes \\
\hline Students & positive & & positive & & positive & negative \\
\hline Non-Students & negative & & positive & & positive & zero \\
\hline $\begin{array}{l}\text { Notes: } R / E=\text { racial/ethn } \\
\text { SES = socio-econom } \\
\text { positive/negative/zer } \\
\text { applicable/moderati }\end{array}$ & $\begin{array}{l}\text { WB=wel } \\
\text { tatus; Ye } \\
\text { individua }\end{array}$ & $\begin{array}{l}\text { eing; J } \\
\text { signific } \\
\text { fect siz } \\
\text { conduc }\end{array}$ & b satisfact & $n ; A A$ & $\begin{array}{l}\text { Affirmati } \\
N / A=n o\end{array}$ & Action; \\
\hline
\end{tabular}

For Negative Well-being and Affirmative Action Attitudes, the moderators identified the particular areas where PD's relationship with the said variables would be stronger, such as levels of measurement, study context, age bracket and sample population. 


\section{Discussion}

There were three aims for conducting a meta-analytic research on the correlates of PD namely a) to examine the average effect of PD on its correlates (antecedents and outcomes), b) to identify if the effect is homogenous across different constructs and c) to explore potential moderators. Further, the study is also expected to identify further gaps in the current literature that could serve as avenues for future research.

All the hypotheses proposed in this study were supported although with differing magnitudes of the main effects. The strongest main effect is PD's link to job satisfaction and the smallest is the relationship of PD with Socio-Economic Status. The directions of the relationships were also consistent with the proposed hypotheses. The subsequent moderator analyses provided additional information on the strength of PD's relationship with some variables. The following are the important findings of the meta-analysis.

\section{The Overall Relationship of PD with its Correlates}

The first meta-analytic finding was consistent with previous studies, wherein results show that identification with one's ethnic/racial group is positively associated with perceived discrimination (Barry \& Grilo, 2003; Evans \& Herr, 1994; Hall \& Carter, 2006; Jefferson \& Caldwell, 2002; Romero \& Roberts, 1998; Scott, 2003; Sellers \& Shelton, 2003). Furthermore, social stratifications may result in the under-privileged group to be more perceptive to discrimination and to have a stronger sense of belonging to the group to maintain positive self-concept. Alternatively, Major et al.'s (2002) review pointed out that individuals who have higher group identification are 
more receptive to stigmatization thus it becomes easier for them to relate their experiences to discrimination.

Second, the negative effects of PD on psychological and physical wellbeing were found to be consistent with existing literature arguing that PD is a stressor that leads to negative outcomes and detrimental effects on the individual's psychological health (Berry, 1990) in various aspects on individual's psychological well-being or health, such as depression (Noh, Beiser, Kaspar, Hou \& Rummens, 1999; Pernice \& Brook, 1996), distress and anxiety (Kessler, Mickelson, \& Williams,1999; Pernice \& Brook, 1996); life satisfaction (Brown, 2001; Liebkind \& Jasinskaja-Lahti, 2000b; Ying, 1996); behavioral problems (Liebkind \& Jasinskaja-Lahti, 2000b; Verkuyten, 1998); and physical health (Krieger \& Sidney, 1996; see review of Harrell, Hall, \& Taliaferro, 2003). Since the source of discrimination is largely external to the self, the individual has to create his/her own strategies to combat this stressor, either through denial or overestimation of the perceived discrimination (Dovidio \& Hebl, 2004) to protect the individual's self-concept. Nonetheless, as evidenced by the findings, these strategies can not totally shield the individual from experiencing negative health outcomes (Cole, Kemeny, Taylor \& Visscher, 1996).

Third, some of the weakest effects were found for the self-esteemrelated measures. The main effect was significant but it was very small, hence, a large portion of the variance remained unexplained. Moreover, the moderator variables used could not explain more on the PD-self esteem relationships since most studies done on self-esteem used in the meta-analysis are the youth sector and student samples. Thus, there is a need for more 
research on contextual variables examining PD-self-esteem relationship. Previous research has already pointed to the influence of ethnic identity (Romero \& Roberts, 2003), the personal and group discrepancy (Bourguignon et al., 2006; Greene et al., 2006) and personal control measures (Moradi \& Risco, 2006). Hence, these psychological variables may influence the extent to which PD and self-esteem are related. These possible moderators are not, however, available for all studies used in the current meta-analysis to be considered as contextual variables.

Fourth, the organizational variables considered in the meta-analysis showed large and small effect sizes for job satisfaction and Affirmative Action attitudes, respectively. It would have been desirable to include more organization variables but there is a scarcity of studies that directly measured PD and related it to similar outcome variables.

Nonetheless, the largest effect size was found for Job Satisfaction showing the significant role that PD plays although there were only a minimal number of studies included in the meta-analysis. Research related to Relational Demography Theory (Tsui et al., 1992) has contributed to the literature on the link of demographic characteristics to attitudes and psychological adjustment outcomes. A qualitative review by Riordan and colleagues (2005) showed that out of the 25 studies linking demographics with job satisfaction, 61\% showed significant results. Moreover, PD has been referred to as a proximate effect of demographic variables in relational demography. Thus, attitudes and behaviours such as job satisfaction are distal outcomes of demographic characteristics (Riordan et al., 2005) since PD is considered an intervening variable between demographics and outcomes. As 
for favourable attitudes towards Affirmative Action programs, the small main effect size means that PD plays a small but significant role in determining favourable attitudes towards Affirmative Action policies.

Fifth, we did exploratory research on the relationship of Socioeconomic status with PD. Socio-economic status has been significantly linked to PD such that those who perceive more discrimination are those in the lower economic status group (Herring, Thomas, Durr, \& Horton, 1998; Taylor \& Turner, 2002). However, other studies have reported different results (e.g, Ensher et al., 2001; Evans \& Herr, 1994; Gibbons et al., 2004, Phinney et al., 1998; Romero \& Roberts, 1998). In this study, higher PD was linked to lower socio-economic status supporting Herring et al.'s (1998) and Taylor and Turner's (2002) findings, although the effect size is small.

\section{The Role of Contextual Variables (Study and Participants' Characteristics)}

There are contextual factors that affect the relationship of PD with the variables in study. Higher PD is associated with higher race/ethnic identity in studies that have used general perceptions scales. This means that frequency scales are not as effective in capturing PD in these studies. Possibly frequency scales do not capture a wide range of PD cognition such that they may be insensitive to a low baseline of overt or covert discrimination, or they may be insensitive to the severity of the experience as viewed by the targets (R. Fischer, personal communication, June 3, 2009). Hence, overall evaluations of discrimination in terms of interval PD scales appear more effective in capturing perceptions about the social environment like organizations as context of discrimination. 
Moreover, higher PD is related to lower ethnic identity in studies that have used multi-level scales, while higher PD is related to lower ethnic identification in studies that have used single-level measures. Hence, variations in results were observed depending on the design of the scales used to measure PD. Similarly, measurements in terms of personal PD and group PD have resulted in non-significant relationships between PD and racial and ethnic identity. These are consistent to the findings of Cassidy et al. (2005) and Phinney et al. (1998). It appears that multi-level measurements capture more target perceptions (i.e., towards peers, superiors, systemic) leading to stronger effects compared to single level scales that have limited scope (R. Fischer, personal communication, June 3, 2009). Hence, future studies should look more closely into this direction.

Results also show that conducting the studies in schools rather than in field settings differs on how racial identity is related to PD. Studies conducted in school settings and involving students suggest that higher levels of PD lead to stronger identification with one's racial/ethnic group, while for studies conducted in the field setting with non-student participants, higher levels of PD result in lower racial/ethnic identification. Participants who are recruited in field studies are more likely to be employees, professionals, organizations/associations members or community members. For these participants, they tend to minimize their identification with the racial/ethnic group as the need to identify with the organizational goals becomes more important.

Often, minorities in organizations are minimal in number such that they are considered as tokens (Kanter, 1977). Because of their group size 
tokens are easily identifiable and become segregated because of their dissimilarity to the majority. The tokenism hypothesis (Kanter, 1977) further explains that tokens are faced with exclusionary treatment hence they tend to be segregated, detached and conform to the stereotypes formed by the majority group instead of proving them wrong. Various studies reviewed by Riordan et al. (2005) discussed that the token individual is faced with the difficult choice of either to underachieve (to minimize majority group's apprehension for him or her as a competitor) or overachieve just to meet everybody's expectations. Therefore, identification with one's own ethnic or racial group becomes a liability and it is downplayed.

Student participants in school settings, on the other hand, still have leeway to express their own views and opinions and they have more support groups that could validate these views towards enhancing their racial/ethnic identification. Clark and colleagues (1999) suggested that contextual supports and the individual coping strategies influence how discrimination affects psychological functioning. Academic commitment and positive school experience have been proven by research to be protective factors for youth adjustment and psychological health outcomes (Hawkins, Catalano, \& Miller, 1992; Petraitis, Flay, \& Miller, 1995). Thus, supportive school environment, teachers and peers can foster mutually supportive relationships which can help the students from target groups contest negative experiences (Patterson, DeBaryshe, \& Ramsey, 1989). Ruggiero and Taylor (1997) added that American youth drew on academic success for their coping strategies to combat the effects of discriminatory experiences such that these youths attribute the negative behaviour to the perpetrators of discrimination and not 
towards themselves. Hence, they were unlikely to blame the discriminatory messages on their personal characteristics.

The moderator analysis showed that the positive relationship of PD with favourable attitudes toward Affirmative Action was maintained - with a higher effect for studies that used multi-level measurements, are done in the school context and involved student or adolescent sample. However, in these studies, there is still significant variability that may be caused by other moderators. There is no significant variability in studies that used single-level measurements, were done in the field contexts and used adult or non-student samples. Hence, the moderators did account for some of the variability in effect sizes. Overall PD is associated with more positive evaluations of Affirmative Action, but this effect is significantly weaker in field contexts and among adults. Thus, the presence of Affirmative Action policies has positive impact for people who have higher discriminatory perceptions since they will display more favourable attitudes towards implementation of these policies. However, favourable attitudes towards Affirmative Action in work contexts are less strongly tied to PD.

Another finding on the test for moderating variables showed differences for age bracket and sample population for the relationship of PD and Socio-economic Status. For adolescents and students, lower Socioeconomic status is associated with higher PD; while for adults and nonstudents, higher Socio-economic status is associated with higher PD. Hence relatively less priveleged adolescents at school perceive more discrimination than peers who belong to high economic status. 
On the other hand, adults who have higher income levels report more discrimination. This was initially explained as minority members who belong to higher economic ladder will experience more discrimination since they will likely be employed, and having jobs increase the likelihood of negative interactions with the majority group. According to relative deprivation advocates, individuals make cognitive comparison that leads to emotions and physical actions (Pittigrew, 2002; Smith \& Walker, 2008). Glick (2002, in Smith \& Walker, 2008) further argues that out-groups are the usual focus of envious prejudice wherein their success elicits reactions of envy, resentments and hostility. Success of the out-group elicits negative emotions since they are viewed as agents responsible for the deprivation hence they lessen the individual's chances of attaining a coveted goal (Glick, 2002, in Smith \& Walker, 2008). Thereby, the majority group members who see well-off minority members may engage in discriminatory attitudes and behaviours due to higher awareness of and resistance against high-achieving minority members with greater aspirations since they pose as competitors for a desired goal.

\section{Limitations of the Study}

The first limitation of this meta-analysis is the file-drawer problem (Rosenthal, 1984) wherein selection of studies was focussed on peer-reviewed journal articles. Although the reference lists of these included articles were subsequently manually scanned for additional studies and requests for further studies were sent to individual authors (21) no unpublished materials were included in the meta-analysis. Hence, there might be some studies looking into PD and related variables that were excluded. 
Due to restrictive time constraint in conducting this research only empirical studies with reported correlations were given attention and were included in the subsequent analysis. The authors of the articles with no correlation tables were contacted and were requested for the needed data. Most responded that they no longer have a copy of their calculations for reasons of relocation, or the co-authors have the copy of the calculations but these coauthors were unreachable. Two authors provided a link to a website where the detailed results were inaccessible to outside users. Further inquiry with them did not yield any response. One author provided a data file which could not be opened probably because of incompatibility of statistical programs. Another author provided a correlation table which was not directly related to the variables currently being studied. Overall, the effort of finding more articles with sufficient data for inclusion was not that successful.

Other limitations of the study can be traced to the inherent methodological weaknesses of meta-analysis (see discussion of Lipsey \& Wilson, 2001). Meta-analysis has been criticized for its systematic and numerical approach to data though this has been viewed by others as strength. Aggregating data from different sources can also lead to comparing "apples and oranges" since studies have their own different characteristics and come from different research contexts. However, this can be addressed by setting specific parameters and introducing variables that could identify sources of heterogeneity in the data just like what was done in this research wherein moderator variables were introduced in the analysis such as research context, age bracket and sample population. 


\section{Suggestions for Future Research}

There are several future directions identified based on the results of this study. First, there is a need to expand in terms of focus on studies in workplaces to further advance the understanding of how PD affects cognition, behaviours and outcomes in the age of globalization. It was noticed in the meta-analysis that there were only a handful of studies looking into organizational effects and job-outcomes. Even the small number of studies on job satisfaction included in the analysis demonstrated a large effect size which means that this is an important variable that should be studied in relation to discrimination. There is a whole range of organizational outcomes to be studied that could further our understanding on how minority groups can effectively cope in the increasing diverse workplace brought about by migration patterns especially at the current trend of increasing competition for skilled human resources. These organizational studies were usually off-shoots of diversity research studies that are attempting to understand and manage an increasing diverse workforce. Global boundaries are said to be disappearing due to increasing migration and advanced technology. More importantly, the reasons for today's migration are more economic in nature. Thus, receiving countries have to exert more effort to attract the best candidates and at the same time implement policies that would help retain these talented and skilled individuals.

Secondly, it was noticed that more focus has been accorded to the negative effects of PD on psychological and physical well-being. Investigation on the positive aspects of well-being (e.g. life satisfaction) should be increased in order to expand our understanding of PD. There are studies that are 
currently citing general well-being measures but they define them in terms of negative outcomes of health, both psychological and physical. These trends in research need to be augmented with the positive measurements of well-being to validate if PD negatively affects positive well-being. If this is confirmed then there will be a holistic view of the overall effect of PD on individual's physical and mental health.

Third, by far studies on specific race/ethnicity are concentrated on African Americans and Latino groups in the Unite States. These groups have a long history of settlement in the United States. Thus, their perceptions might differ from first generation and/or second generation immigrants who have recent periods of settlements in their host countries. Increased attention is warranted on relatively new settlers as a relevant issue in the age of globalization.

Fourth, discrimination studies have traditionally focused on Black or African American vs. White issues. In the meta-analysis, other study characteristic like ethnicity and country of study could have been useful moderators in the relationships between PD and its correlates. However, they were not included in the final analysis since the distinction of ethnicity in the studies by their authors was not specific and was not varied enough (e.g. mostly focusing on Black or African groups and a few on Latino/Hispanic groups) nor systematic enough (e.g. diverse groups can be composed of totally different ethnicities) for them to be categorized as specific ethnicities that could be studied. Thus, additional studies are needed for other populations who show different demographic characteristics (e.g. various ethnic groups of Asians). Hence, future research should attempt to include more analysis on 
other ethnic groups when making comparisons between majority and minority groups.

Fifth, the majority of the studies gathered for the meta-analysis were based in the United States (82\%) with a growing number of studies from Europe. This is not surprising since the United States has a long history of immigrant settlement starting from the time of British and other European nationals' colonization of America. However, the $20^{\text {th }}$ century was marked with changes in migration trends that shifted from traditional destination countries like United States, Europe and Canada to new countries of destination like Australia and New Zealand. Additional studies, therefore, are needed in new immigrant destination countries like Australia and New Zealand wherein increasing diversity is a growing organizational, social and political concern. The differing perceptions of host nationals and immigrants in these countries are worth investigating especially in the context of discrimination.

Finally, it was observed that variables considered in the study were not directly referred to as antecedents and outcomes of PD. Usually, these variables are considered as correlates or covariates. Hence, future studies should look into the careful examination of constructs as whether they are more important to be considered as antecedent or outcome to isolate variables that have the most important impact on PD and vice versa. 


\section{Summary and Conclusions}

In conclusion, our findings show that research on perceptions of discrimination should pay special attention to different contextual variables that could further explain the nature of the relationships between and among the correlates. Gaps in research that were identified led me to conclude that there is a need to conduct research on organizational outcomes on specific target groups (e.g. immigrants). Attention accorded to PD's effects on organizational outcomes of immigrants in new countries of destination is almost non-existent. Increasing the number of studies in these areas is like hitting two birds in one stone - organizational outcomes and immigrants, both of which are less studied in the psychological literature. Furthermore, to advance the knowledge on these job outcomes, it is deemed that health and well-being variables should also be studied in the organizational context. Much of the existing studies done on PD in relation to psychological and physical well-being were done by scholars interested in acculturation and youth research. It is about time that organizational psychology should contribute more studies on this area. The succeeding chapters hope to address these concerns and aim to advance the current literature of PD with variables that are relevant to the current times. 


\section{Chapter 3}

\section{A RESEARCH FRAMEWORK FOR THE STUDY OF PERCEIVED DISCRIMINATION IN THE WORKPLACE}

\section{Introduction}

The results of the meta-analysis in Chapter 2 demonstrated that there is a need to increase the level of psychological research on perceptions of discrimination (PD) in workplace settings. This chapter addresses the abovementioned concern by specifically looking into the issues related to discrimination in organizations or places of work.

The Human Rights Act 1993 (HRC, 2000) considers a discriminatory act as unlawful if it has a ground or basis (e.g. race, ethnicity) and done in a particular context (e.g. employment, education, housing and accommodation). To satisfy the first requirement, this thesis focuses on race- or ethnicity-related issues as grounds for discrimination. Determining a specific context, the second requirement, proves to be tricky due to the vast and varied domains of psychological research. The results of the meta-analytic study in the previous chapter, however, have directed me to one pertinent direction.

Organizations or workplace settings, a very relevant area or context of discrimination especially in the age of worldwide economic integration, was identified in the previous chapter as needing some attention in terms of empirical research. This chapter thereby addresses this gap in research by specifically looking into factors related to PD in organizations from the targets' perspective.

Workplace discrimination is defined as "unfair and negative treatment of workers or job applicants based on personal attributes that are irrelevant to 
job performance" (Chung, 2001, p. 34). Even if legislations are in place to shield people in the workplace against discrimination due to race, colour, national origin, etc. (Phan \& Kleiner, 1999), an individual can already be discriminated against even before he or she sets foot in the workplace. Furthermore, studies show that even if an individual has long been engaged in a job, he or she can be a target of discrimination as a consequence of his/her race/ethnicity (Deitch et al., 2003). Hence, target groups can be discriminated against at different stages of employment.

\section{Access and Treatment Discrimination}

Levitin et al. (1971) identified two components of discrimination experienced in the organizational setting that illustrate the realities faced by disadvantaged groups during the pre-employment stage and on-the-job experiences. Access Discrimination refers to the unequal treatment that an individual will be subjected to during the recruitment and selection procedures such as denial of a preferred job and lower starting pay. Treatment Discrimination, on the other hand, would be the conditions of differential treatment that limit the individual from realizing his/her full potential in the workplace (e.g., promotions or career advancement). These two components were further investigated by Brown and Ford (1977) in their study of discrimination in employment of Black Americans. They found that Black Americans with MBA (Masters in Business Administration) face greater disadvantages related to access and treatment discrimination compared to their White counterparts. Chung (2001) also referred to Levitin et al.'s (1971) components when he proposed a model for work discrimination and coping strategies of lesbian/gay employees. This distinction between job-entry 
(access) and on-the-job (treatment) experiences are less directly referred to in the literature but are alluded to in various studies.

Hence, discrimination can happen when people are still on the stage of seeking employment or securing employment (Stewart \& Perlow, 2001; Wilson, Gahlout, Mouly, \& Liu 2005). This form of discrimination happens when migrants are being discriminated against by potential employers and formal institutions during job selection or when recognition of overseas qualifications is being sought (e.g. Jasinskaja-Lahti et al., 2007; Selvarajah, 2004; Stewart, 2005; Ward \& Masgoret, 2007). These studies present instances that may be considered as covert practices of discrimination in recruitment guised as normal organizational practices. Most employers or human resource practitioners in these studies prefer applicants who are similar to their characteristics thereby ignoring others who may have the same, if not better, skills and abilities than their chosen candidates but differ in their ethnic background.

Furthermore, studies show that even if an individual has long been engaged in a job, he or she can be a target of discrimination as a consequence of his/her race/ethnicity (e.g., Deitch et al., 2003). The pervasiveness of discrimination in the workplace has long been documented in various studies all over the world. In a US nationwide-wide study, Black Americans who were working in pre-dominantly White organizations perceived discrimination in the form of difficulty in advancement, underemployment, less privileges and harder discipline (Bowman, 1991). A much later study by Deitch and colleagues (2003) showed that Black American employees perceived trivial yet persistent mistreatment and unfairness more than their White counterparts. 
Canadian studies similarly report that visible minorities (non-White) perceive greater discrimination than those from White ethnic background (Dion \& Kawakami, 1996). Discrimination also happens regardless of position titles and levels in the organizational hierarchies. Even the performance ratings of employees with MBA degrees or those in managerial positions are still affected by discriminatory attitude as a function of their racial background (Brown \& Ford, 1977; Leeming \& Baruch, 1998; Levitin et al., 1971; Tomkiewez, Adeyemi-Bello \& Johnson, 1999).

\section{Formal and Informal Employment Discrimination}

Formal and informal discrimination were two forms of discrimination identified by Levin and Leonard (1984). These two forms were then used by Chung (2001) in his model of work discrimination and coping strategies of lesbian/gay employees. Formal discrimination was described as formal or institutionalized processes that restrict target groups' access to certain outcomes such as job mobility like promotions, salary increases, more job responsibilities and other procedures related to hiring and firing minority employees. Secondly, informal discrimination pertains to unofficial policies or practices that allow harassment and derogation of minority employees. This includes verbal harassments such as gossip, taunts, and ridicule as well as nonverbal forms of harassment such as stares, ostracism and damage to personal belongings (Levin \& Leonard, 1984).

\section{Objective Versus Subjective Measures of Discrimination in the Workplace}

The relative paucity of studies on PD in the workplace setting was pointed out in the meta-analysis and in the literature reviews of previous 
chapters. Further, understanding PD from the perspective of the targets and the resulting effects on job-related success, physical and mental health is being addressed by a few though growing number of studies, mostly in gender studies (e.g., Gutek et al., 1996; Mays, Coleman \& Jackson, 1996) and in the diversity management literature (e.g., Ensher et al., 2001; Sanchez \& Brock, 1995). What is common in the current discrimination literature in the contexts of organizations, minority and gender studies is the focus on economic consequences such as differentials in employment opportunities, promotions, wages, and residential segregation (Cordero-Guzman, 1992; Feagin \& Eckberg, 1980; Frieze, Olson, \& Good, 1990; Kahn \& Krosby, 1985; Badgett, 1995; Madden, 1985; Tienda, Donato, \& Corder-Guzman, 1992; Phelps, 1972). Furthermore, early studies on workplace discrimination have focused on data that are readily accessible like compensation thus neglecting other areas of employment (Balser, 2000).

It is noteworthy that the majority of studies on ethnic-racial discrimination in the workplace have revolved around economic consequences of workplace discrimination such as wage issues and unemployment (Darity \& Goldsmith, 1993; Elmslie \& Sedo, 1996). Workplace inequalities reflected in objective measures like unemployment rates and wage differentials have been focal issues for measuring the extent of workplace discrimination especially in highly diverse areas with long history of migration such as North American countries and European countries. Significant gaps in income of minority employees when compared to White employees were found in some professional occupations (Hirsh \& Schumacher, 1992). 
Similar findings were reported wherein Black Americans have historically been receiving lower income than White Americans and the gap persists over the years (Dovidio \& Gaertner, 1986). The 2006 United States Census Bureau (DeNavas-Walt, Proctor, Smith, USCB, 2007) reports a 36\% difference in income for White Americans $(\$ 50,673)$ versus Black Americans $(\$ 31,969)$. It has also been noted that first generation immigrants always find employment in the host country a challenge thus making their unemployment rate much higher compared to the host nationals' (Jasinskaja-Lahti et al., 2007). Further, studies show that the long-term economic effects of discrimination in relation to prolonged unemployment lead to deterioration of skills, learning abilities and motivation in the United States (Elsmlie \& Sedo, 1996) and wage differentials that penalize even the second generation minority in Great Britain (Blackaby, Leslie, Murphy, \& O’Leary, 2005).

Similarly, a recent review on inequities in the labour market by Podsiadlowski and Ward (forthcoming) mentioned that the unemployment rate of foreign born residents was 5.3\% higher than that of native-born Austrians based on a 2006 Austrian census. Moreover, their review also described foreign non-Western nationals in the Netherlands as three times more likely to be unemployed than the native-born Dutch. Canadian studies focusing on earnings and employment statistics also show that race/ethnic disparities exist in the Canadian labour market particularly for foreign-born workers (e.g. Baker \& Benjamin, 1997; Pendakur \& Pendakur, 1998; Ornstein, 2000; Reitz, 2001).

More recent migrant destinations such as Australia and New Zealand also reflect similar trends in terms of unemployment disparities. New Zealand 
statistical reports show that unemployment rates for immigrants are $1.9 \%$ higher than native-born (Statistics New Zealand, 2002). The report also show that unemployment rates for New Zealand immigrants from non-English speaking countries is $12.65 \%$ higher than migrants from English-speaking countries Correspondingly, Australian figures show that unemployment rates of migrants from non-English speaking countries is $12.3 \%$ and $12.5 \%$ higher than for native-born Australians and migrants from English-speaking countries respectively.

Although there is a marked trend showing the disadvantaged economic position of racial/ethnic minority groups, a growing number of studies have reported little or no support for majority groups' disadvantaged position in specific contexts. Thus, native-born groups' income has been found to be comparable to minority groups. For example, De Silva (1997) found no significance difference in the income of Canadian Whites and Non-White or Non-Caucasian minority groups (termed as 'visible' minorities by a Canadian legislation) who were similarly qualified. Palameta (2004) showed no significant difference in income of Canadian Whites and non-Whites. Other studies also found no conclusive results in comparing total ethnic minority groups with that of local-born but found specific ethnic group differences. For instance, Hum and Simpson (1999) found income differences between Black and White Canadian employees but no significant difference was found in the income of other minority groups compared to White Canadians. Swidinsky and Swidinsky (2002) examined the income gap of native-born Canadians with that of non-White minority groups and reported a significant difference for the income of minority men compared to majority men (for Black 
employees, males and females had lower salaries). In the United States, the annual average income of Asian workers is $22.5 \%$ higher than that of White Americans (DeNavas-Walt et al., 2007).

Linking income disparity to discrimination has been prevalent since studies have found that minority groups claiming to be victims of discrimination usually have lower wages than host nationals (Herring et al., 1998). However, the apparent inconclusive results of measuring the extent of workplace discrimination in terms of "objective" manifestations point to the importance of investigating the "subjective" aspect of workplace discrimination through the exploration of discriminatory perceptions (Banerjee, 2008). Unfair treatment directed to target groups may not always be reflected in economic outcomes like earnings. Furthermore, Barnerjee (2008) believes that target groups may acknowledge their economic disadvantage without attributing it to discrimination, and income inequities in organization may be tempered by affirmative action programs and other legal influences. Thus employees may continue to perceive discrimination from other subtle forms of discriminatory factors in the workplace.

In contrast to objective measures of discrimination, perceptions have been found to have harmful effect on attitudes, behaviours and health since people consider their perceptions as representations of reality (see metaanalytic study in Chapter 2; Banerjee, 2008). Perceptions of discrimination, in the workplace context, reflect employees' beliefs that they are unfairly treated due to their personal attributes. Employee perceptions are deemed important as people's beliefs, regardless of whether they are accurate reflections of 
reality or not, affect work behaviours (Barack, Cherin, \& Berkman, 1998; Eisenberger, Fasolo, \& Davis-La Mastro, 1990).

Perceptions of discrimination in the workplace have been found to be negatively linked to organizational outcomes such as job satisfaction, organizational commitment, organizational citizenship behaviours (Deitch et al., 2003; Ensher et al., 2001; Sanchez \& Brock, 1996), positively associated with work tension (Sanchez \& Brock, 1996), inversely related to well-being (Deitch et al., 2003; Jasinkaja-Lahti et al., 2007; Mays et al., 1996; Pavalko, Mossakowski, \& Hamilton, 2003) and result in detrimental legal consequences like grievances and lawsuits (e.g. Allen \& Keaveny, 1985).

Despite the apparent importance of looking into perceptions of discrimination as a major source of negative outcomes for employees, only a handful of studies have taken interest in this area as revealed in the metaanalytic study in Chapter 2. Various studies have also discussed different conceptualizations of work discrimination either in relation to the consequences of perceived workplace discrimination (e.g. Deitch et al., 2003; Ensher, et al., 2001; Foley \& Kidder, 2002; Sanchez \& Brock, 1996) or its antecedents (e.g. Balser, 2002; Banerjee, 2008). Moreover, it has been noted that there is no comprehensive framework that offers an integrative perspective to the various conceptualizations of work discrimination (Chung, 2001). To address this gap in research, my thesis contributes to the literature by presenting a research framework that examines the correlates of PD in the workplace by looking at both antecedents and consequences/outcomes of PD in the workplace from the targets' perspective (i.e. those who perceive the unequal treatment in the workplace). 


\section{Antecedent-Outcome Framework of PD in the Workplace}

This chapter presents a conceptual framework that guides the succeeding studies in the examination of the processes involved in the perceptions of workplace discrimination. The proposed framework of this thesis (see Figure 3.1) attempts to capture a holistic view of PD process. This is in line with the overall research aim of enriching the growing literature on perceptions of discrimination (PD) from targets' points of view and to examine the antecedents of their perceptions and the consequences of PD on their personal outcomes. This particular approach has been described by Schnittker and McLeod (2005) as focusing on "upstream" mechanisms that look at antecedents/influences and "downstream" mechanisms that focus on consequences/effects.

The conceptual framework is intended to be used solely as a guide in looking at the three components of the framework represented by three separate studies. These studies are conducted to investigate each individual aspect namely, a) nature of PD; b) antecedents of PD; and c) outcomes of PD. There is no intent to link the findings of the three components of the framework (which may qualify for moderation or mediation in future studies) since at this point most of the relationships being tested in the separate components of the framework are exploratory in nature.

This thesis is specifically looking at individual-level PD with the following specific objectives 1) to investigate what are the emerging discriminatory perceptions in the workplace of selected target groups and to construct if not adapt a PD measure relevant to the workplace context that would be consistent to the selected targets' perceptions; 2) to take the 


\begin{tabular}{|l|}
\hline Proximal Influences \\
on Perceptions \\
(Perceiver's \\
Characteristics)
\end{tabular}

Distal Influences on

Perceptions

(Organizational

Characteristics)

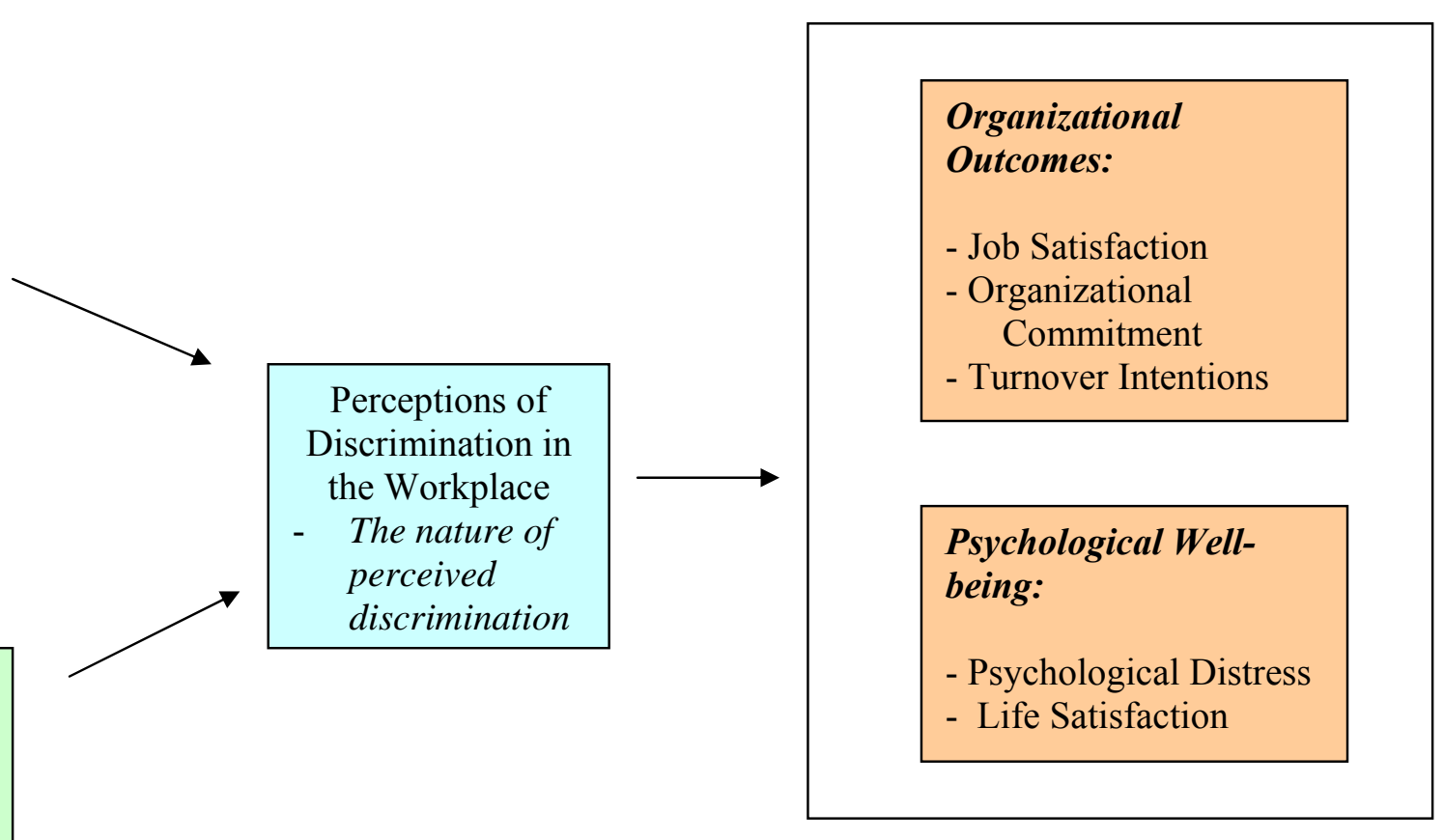

Figure 3.1 Antecedent-Consequence Research Frameowork on Perceived Discrimination in the Workplace 
"upstream" approach by looking into antecedent factors or examining what influences discriminatory perceptions; and 3) to follow the "downstream" approach through the examination of the links of PD on attitudes, behaviour and health with specific focus on organizational outcomes and psychological well-being.

\section{Conceptualization of PD in the Workplace}

from the Target's Perspective

The middle section of Figure 3.1 pertains to the nature of discrimination as perceived in the workplace by targets. A significant portion of this research is devoted to the investigation of the current conceptualizations about $\mathrm{PD}$ and existing measurements used to gauge perceptions using qualitative and quantitative research methods. Combining qualitative and quantitative methods was a deliberate strategy used in this thesis to make best use of the advantages of and to minimize the disadvantages of both research methods. The use of both methods was put into action in the investigation of the nature and existing measurements of PD.

As presented in Chapter 2, discrimination has been studied in varying fields and in different contexts. Discrimination and the perceptions of it have been regarded as consequences of social comparisons with similar others and with dissimilar others according to Social Identity Theory (Tajfel \& Turner, 1979; 1986) and derivative models like self-categorization theories and Relational Demography Theory (Tsui et al., 1992). Discrimination has also been regarded as a social stressor for minority groups (Lazarus \& Folkman, 1984; Cassidy et al., 2005). Perceived discrimination and experiences of discrimination related to race/ethnicity have also been assessed in different 
approaches such as individual versus group level, lateral, vertical and systemic levels, and by frequency of occurrence.

Discrimination as a social phenomenon is evolving and has different meanings in different contexts. In the workplace context, the conceptualization of discrimination will be explored if aligned with but not limited to the previously identified two phases (access and treatment) and two forms (formal and informal). Race/ethnicity-related perceived discrimination in the workplace will be investigated through an unobtrusive method. First, preliminary questions in the planned qualitative study (Study 2) will revolve around possible avenues of discrimination without directly mentioning the word "discrimination". Thus, respondents will not be confined to any predetermined definition of discrimination in the workplace. A descriptive approach is appropriate since no prediction of cause and effect is intended at this point. Rather I am looking for descriptions and explanations of discrimination since potential reasons of the behavior are difficult to manipulate, and factors are difficult to extract from the phenomenon (Birzer \& Ellis, 2006; Merriam, 1988).

At the same time, a review of the current measurements of race/ethnicity-related $\mathrm{PD}$ specifically in the organization setting will be conducted to validate the perceptions that will be generated from the qualitative study. Selection of items that will measure PD will also not directly mention discrimination. Hence, the nature of PD that this research intends to capture is a direct definition from the experience and cognition of the participants - employees in general and immigrants in particular. In the initial investigation on the nature of $\mathrm{PD}$, immigrants' perspectives are the central 
focus of the investigation since immigrants embody multiple characteristics that are susceptible to discrimination. Immigrants have multiple facets in their demographic characteristics that can be simultaneously investigated such as their immigrant status, race/ethnicity, age and gender. Thus this multipledemographic effect is deemed important in investigating targets of discrimination in the workplace like skilled immigrants and is considered as also a step-forward to the on-going investigations done for conceptual models like the Ethnicity Prominence Hypothesis and the Double Jeopardy Hypothesis as described by Levin, Sinclair, Veniegas and Taylor (2002).

\section{Antecedents of Perceived Discrimination}

On the left portion of the framework (Figure 3.1) are the antecedents of PD that are assumed to influence how individuals internalize actions as discriminatory. An individual's appraisal of events as discriminatory or an environment as perpetuating discrimination largely depends on the perceiver's inherent and socially ascribed characteristics and how his/her surrounding environment has institutionalized discriminatory practices. Hence, the understanding of antecedents of PD using the target's perspective can be distinguished either through group categorizations due to inherent and ascribed characteristics or through situational-induced influences on perceptions (Pinel, 1999).

The antecedents are thereby subdivided into proximal influences and distal influences. First, the proximal antecedents or factors are the inherent or ascribed characteristics of the targets of discrimination. These factors are internal to the individual such as demographic characteristics. The second type of antecedents is the distal factors or contextual factors. These factors are not 
inherent to the individual or external to the individual yet they influence how targets perceive discrimination. In the context of workplace discrimination, the factors that will be considered are processes and structures within workplaces or organizations.

\section{Proximal Antecedents of PD}

Central to this thesis is the examination of the influence of race/ethnicity as a demographic characteristic on PD. Ethnicity, also referred to as surface-level diversity, is defined as "differences among group members in overt, biological characteristics that are typically reflected in physical features" (Harrison, Price \& Bell, 1998, p.97). Moreover, ethnicity similar to race, is also considered as a social categorization (William, 1997). Secondary to race/ethnicity, other demographic variables such as age, gender, income (as an indicator of SES [socio-economic status]), and country of birth (immigrant status vs. native-born) will be considered in this thesis to determine if these factors are also salient in influencing perceptions of discrimination. It has been recognized in the literature that women (Araujo \& Borrell, 2006; Konrad \& Spitz, 2003; Gutek, Cohen \& Tsui, 1996; James, Lovato, Cropanzano, 1994; Pavalko et al., 2003) and older adults (De Castro, Gee, \& Takuchi, 2008; Gee, Pavalko \& Long, 2007) encounter more workplace discrimination.

Among the demographic characteristics, race and gender have been identified as most prevalent grounds of discrimination in American society (Birzer \& Ellis, 2006). In organizational research two competing hypotheses exist about which is more salient for individuals - gender, ethnicity or both. The Ethnic-Prominence Hypothesis proposes that regardless of gender, group membership by ethnicity will be more salient for the perceptions of 
discrimination of minority groups (Levin et al., 2002). Further, Relational Demography Theory (Tsui et al., 1992) and Social Identity Theory (Tajfel \& Turner, 1979; 1986) have explained how ascribed identities such as ethnicity can be relevant in organizational settings thereby demonstrating that social identities and social categorizations are important in the workplace context (Terry, 2003).

In contrast, the Double-Jeopardy Hypothesis suggests that ethnicity and gender have joint effects in influencing perceived discrimination. This theory assumes that gender plays an additional role in the experience of discrimination. Belonging to a minority group and being female have been referred as double status disadvantage by scholars who have studied African Americans (Wilcox, 1997; Weinberg, 1998). For instance, ethnic-minority women, who can be discriminated on the bases of gender and ethnicity, will have greater disadvantage than ethnic-minority men, White women and White Men (Levin et al., 2002).

Yet another approach is put forward by Social Dominance Theory proposing that ethnic-minority men face more discrimination because they are viewed more as a threat to the superiority of the majority group than minority females (Pratto, Sidanius \& Levin, 2006). Thus, the Subordinate Male Target Hypothesis argues that subordinate males are generally the targets of arbitrary discrimination that are institutionalized in the labour market, justice system, housing and retail market (Sidanius \& Pratto, 1999).

Focusing on material conditions, the material and status conceptualization of Socio-economic Status generally points to a better wellbeing of individuals if they are categorized as higher Socio-economic Status 
due to the monetary advantages as well as higher social status affiliations (Schnittker \& McLeod, 2005). In Chapter 2, the meta-analytic study on PD and its correlates found a small significant effect size on Socio-economic Status which means that higher PD is negatively related to Socio-economic Status.

The last demographic variable to be studied is country of birth which is viewed as an important variable to consider in studies that look into plural societies. The current setting of the study is New Zealand which is historically an immigrant country. Aside from ethnic differences, the status of being native-born and being an immigrant have different social consequences for the individual. For instance, immigrants in Canada face the greatest challenge especially those who are recent arrivals (Ademir \& Skuterud, 2005; Bloom, Grenier, \& Gunderson, 1995). Thus, I would like go beyond the usual individual characteristics considered in research studies by examining factors beyond the common two group memberships (i.e. ethnicity and gender) of an individual. A third relevant factor considered here is the immigrant status. It has been pointed out in previous studies that some minority groups especially women can be potential targets for "multiple discrimination" (Ghosh, 1984; Moghaddam \& Taylor, 1987; Taylor, Wright, Moghaddam \& Lalonde, 1990). Exploring the demographic characteristic of being an immigrant or nativeborn is intended to add to the growing literature focusing on "multiple disadvantages" faced by immigrants. This triple-demographic effect has not been clearly examined in the existing multiple group membership literature in the organizational setting and is worth exploring in this current research. Thus, 
it is assumed that immigrant status, ethnicity and gender have individual and combined effects in predicting perceptions of discrimination.

One of theoretical models that explain the importance of demographic variables in the PD process is Relational Demography Theory (Tsui et al., 1992). Organizational psychologists refer to Relational Demography Theory to explain the propensity of individuals to compare the extent of the similarity or dissimilarity of their demographic characteristics with that of their coworkers in their organizational unit. Relational Demography Theory explains that specific demographic characteristics of individuals (e.g. race, ethnicity, gender) become the bases for comparison. The extent of the individuals' similarity or dissimilarity to the comparison group can affect their workrelated attitudes and behaviours. The basic tenet of Relational Demography Theory assumes that dissimilarity negatively affects individuals' outcomes, such that increased demographic differences results in PD. Most of the existing studies using Relational Demography Theory directly test the relationship of demographic differences with organizational attitudes and behaviour (e.g. Jackson, Brett, Sessa, Cooper, Julin, \& Peyronnin, 1991; Riordan \& Shore, 1997; Tsui et al., 1992; Zenger \& Lawrence, 1989). These studies have linked demographic differences to organizational outcomes such as job satisfaction, organizational commitment, turn-over, perceptions of rewards, group cohesiveness and other organizational outcomes (see review of Riordan et al., 2005). In contrast, studies using demographic characteristics as possible antecedents of PD are not common. In studies that directly study PD, demographic information is usually used as control variables in predicting outcomes. 


\section{Distal Antecedents of PD}

This thesis also acknowledges that there are environmental factors that could affect cognitions and perceptions aside from the individual's own characteristics. One of these distal factors is the individual's immediate working environment which could present an array of interrelated stimuli that affect an individual's perceptions. In the context of workplace discrimination, distal factors such as the organizational structures can either enhance or reduce the incidence of discrimination (Gelfand, Nishi, Raver \& Schneider, 2005).

Aycan (2000) pointed out the scarcity of studies relating formal organizational structures to job-related attitudes. Structures such as policies, practices, procedures and regulations define what organizations value most. The organizational structures can have immediate or long-term effects on employees' cognitions, emotions, and behaviours (Baron \& Pfeffer, 1994). However, Aycan (2000) posits that relationships between structures and attitudes are not direct but are mediated by perceptions of employees. The extent to which these organizational-level structures are open to diversity or the increased awareness of the advantages of having diverse employees will have an effect of how the employee perceives his/her conditions within the organization.

Gelfand and colleagues (2005) proposed the Systems Model of Discrimination (SMD) at the organizational level as an endeavour to understand how discrimination can be minimized or increased within the organizational internal and external processes (see Figure 3.2). They elaborate that an organization's overall performance is influenced by both internal and external environments. Macro-level legal and political structures that mandate 


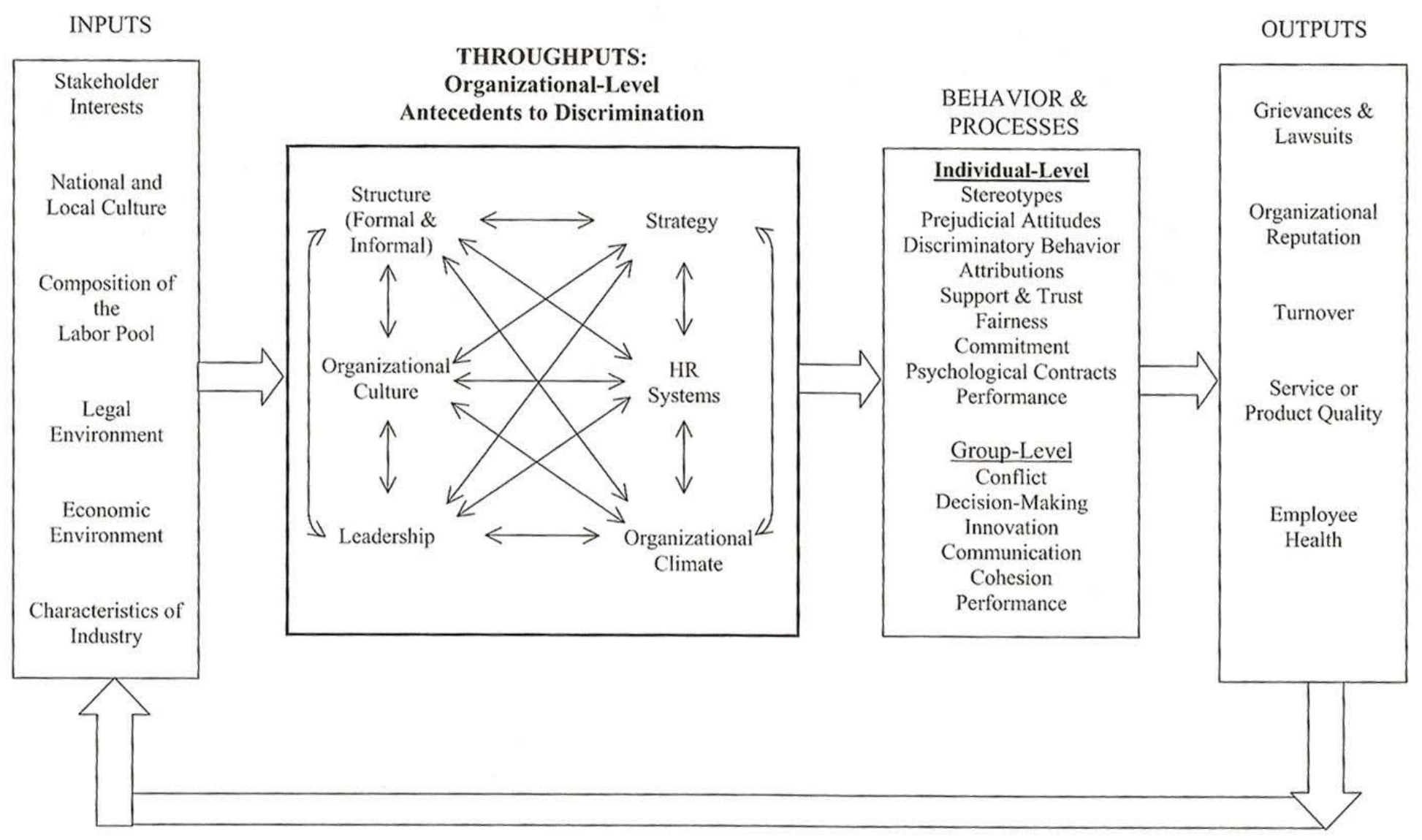

Figure 3.2 The Systems Model of Discrimination (SMD) by Gelfand et al. (2005) 
the allocation of resources form part of the organization's external environment that in turn affects the internal environment. This thesis will not refer to the external structures that influence organizations. Rather, the focus will be on the influences of the organization's internal environment on employee perceptions.

The SMD model depicts six organizational-level systems which individually or collectively, are considered contexts of discrimination within the internal environment of the workplace. These are the organization's structures, organizational culture, leadership, strategy, human resource systems and organizational climate. Descriptions of the systems by Gelfand et al. (2005) that are relevant to this thesis are presented below.

\section{Structures.}

Organizational structures are classified into formal structures and informal structures. Formal structures pertain to organizational mechanisms such as hiring and promotional opportunities. These structures can perpetuate discrimination if they hinder opportunities on hiring and advancement for disadvantaged groups such as minorities and women in organizations. If these disadvantaged groups cannot penetrate their preferred higher level positions in the organization, homogeneous groups within the organizational or in the managerial levels may represent discrimination in the workplace (Bennet, 2002; James, 2000). Informal structures, on the other hand, relate to workplace interpersonal relationships. Baron and Pfeiffer's (1994) review of previous studies concluded that a very important source of satisfaction at work is social relations. Looking at informal structures is parallel to what Embedded Intergroup Theory (Thomas \& Alderfer, 1989) proposes, namely it is 
important to look into the intricate network of organizational relationships (i.e. individual vis a vis supervisors, co-workers, organization itself) for an understanding of PD. Ibarra (2003), however, contends that minorities would have less access to these social networks since the latter are dictated by sociocultural similarities (e.g. preference for those with majority characteristics).

Gelfand's et al.'s (2005) categorizations of these structures also parallel previous conceptualizations by Levine and Leonard's (1984) distinguishing between formal and informal discrimination. Thus, formal discrimination may be embedded in organizational policies related to hiring, compensation, benefits and the like while informal discrimination are manifested in relational dynamics, interactions or work atmosphere. Dovidio and Hebl (2005), described the same two forms of discrimination as formal discrimination (covert forms of behavior that are legally sanctioned by hiring, promotion and firing policies) and informal discrimination as behaviorally implicit interpersonal cues (e.g., lack of warmth, less eye contact).

\section{Human resource systems.}

These are practices related to recruitment and selection, performance appraisals, benefits, training and grievance procedures that can function as institutionalized mechanisms for perpetuating discrimination from recruitment to advancement in the job. Levitin et al. (1971)'s access and treatment discrimination are also relevant in these structures since discrimination may be institutionalized from hiring procedures to advancement opportunities. In addition, hiring policies such as Affirmative Action Programs (AAPs) can have dual effects in terms of minimizing access discrimination at the same time resulting in treatment discrimination of the individuals hired under AAP. 
Thus, it is important that such programs should be properly communicated and supported with evidence of the candidate's competence. Lastly, hiring practices based on informal networks are also a source of discriminatory practices since not all groups may have equal access to such networks (Ragins \& Sundstrom, 1989).

\section{Organizational culture.}

The SMD model describes organizational culture as the prevailing practices in the organization based on the shared beliefs, values and outlook. These shared beliefs and values can be a source of subtle forms of discrimination. The culture can hold certain stereotypes about preferred employees, such that, individuals who do not possess the stereotypic traits are less valued. Resulting behaviours can be discriminatory such as directing inappropriate jokes (Siehl \& Martin, 1988) towards the devalued individuals. Cox (1994) nevertheless argued that a good combination of weak and strong organizational culture values can be used to eliminate discrimination. For instance, he pointed out that the organization's strong belief towards positive aspects of diversity and less importance given to individual styles thereby creating greater flexibility to the culturally deemed acceptable behaviours are a good combination to reducing discriminatory organizational cultures.

\section{Organizational climate.}

Organizational climate is described as the expression of the organization's shared culture. Thus, if the organization has a shared belief about the advantages of diversity, then the resulting organizational climate favours a positive climate for diversity. Hicks-Clarke and Iles (2000) found 
that perceptions of diversity climate are related to organizational commitment, job satisfaction and career-satisfaction.

\section{Strategy.}

Business strategies embracing the advantages of diversity and the culture of inclusiveness have been shown to result in decreased discriminatory behaviours. Programmes such as Equal Employment Opportunity (EEO), public statements of the importance of diversity can be integrated into the organization's strategic plans to emphasize the relative importance of these programs to the organization. It has been shown by Cox, Lobel and McLeod (1991) in their value-in-diversity hypothesis that organizations pursuing innovation strategies favour schemes that value inputs from a diverse workforce.

\section{Consequences of Perceived Discrimination}

The third specific objective of this thesis is to contribute to a broader understanding of how perceptions affect individual outcomes in the workplace settings. The right hand portion of Figure 3.1 represents the organizational and psychological well-being outcomes that will be included in the analysis as consequences of PD.

\section{Organizational Outcomes}

The meta-analysis in Chapter 2 has shown that only a handful of studies have directly linked perceptions of discrimination to organizational outcomes. However, existing research shows that PD in the workplace have been directly linked to low job satisfaction (Deitch et al., 2003; Ensher et al., 2001; Foley \& Kidder, 2002; Sanchez \& Brock, 1996), low commitment to 
organizations (Ensher et al., 2001; Sanchez \& Brock, 1996) and higher intentions to leave the organization (Balser, 2002).

Without reference to PD, Relational Demography Theory studies have directly linked demographic characteristics to organizational and personal outcomes. Previous studies of Relational Demography have obtained significant relationships between factors that measure "being different" from others (e.g. race or ethnicity) and outcomes like job satisfaction, less commitment in organizations, turn-over intentions, depression and so on (see review of Riordan et al., 2005). Recently, proponents of Relational Demography have acknowledged the increased importance of looking at PD as a proximate outcome of demographic variables together with other factors as distal outcomes. Thereby a call for future research on this approach was issued by Riordan et al. (2005).

\section{Psychological Well-being}

Moreover, most studies in the organizational setting focus on jobrelated and career outcomes and fail to consider individuals' health and overall well-being (for some exceptions see De Castro et al., 2008; Mays et al., 1996). Based on the meta-analysis in Chapter 2, PD has been linked to psychological and physical health outcomes mostly in social psychology, acculturation research and gender studies but more focus is needed in organizational contexts. Results of studies show that PD is significantly related to psychological and physical well-being such as depression, distress, anxiety, life satisfaction, and physical health (e.g. Meta-analysis in Chapter 2; Bourguignon et al., 2006; Brown, 2001; Finch, Hummer, Kolody \& Vega, 2001; Finch, Kessler, Mickelson \& Williams, 1999; Kolody \& Vega, 2000; 
Liebkind \& Jasinskaja-Lahti, 2000; Krieger \& Sidney, 1996; Noh, Beiser, Kaspar, Hou \& Rummens, 1999; Pavalko et al., 2003; Pernice \& Brook, 1996; Verkuyten, 1998; Ying, 1996). Thus, an important addition to the organizational literature is the inclusion of measures of the negative and positive aspects of psychological well-being like psychological distress and life satisfaction respectively, and linking them to perceptions of discrimination.

\section{Summary}

Overall, this chapter broadly reviewed aspects of the literature on workplace discrimination. Although organizational research has been around for several decades now, some aspects of PD in the workplace have yet to be explored. It appears that there is a need to increase the amount of research in the area of the subjective side of perceived workplace discrimination. Furthermore, there is also a need for further research on the effect of PD on job outcomes based on the minimal studies gathered in the meta-analysis. There is also a gap in research in terms of psychological well-being of employees.

All these concerns will be addressed by the proposed AntecedentOutcome Framework of PD in the workplace. The investigation will start on the understanding the nature of $\mathrm{PD}$ in the workplace and constructing instrument(s) that will capture the conceptualization of PD as examined in the succeeding studies. Furthermore, to provide a holistic view of the PD phenomenon, this thesis focuses on the simultaneous investigation of the antecedents of PD in terms of personal and environmental influences by 
examining the criterion variables immigrant status (native-born or immigrants), ethnicity, gender, age and influences of organizational structures. Finally, the framework will look into the investigation of PD's impact on organizational outcomes and psychological well-being. Overall, the upstream and downstream examination of the PD in the workplace phenomenon is intended to address a number of gaps in the literature. 


\section{Chapter 4 \\ THE NATURE OF PERCEIVED DISCRIMINATION FROM THE IMMIGRANTS' PERSPECTIVE: A QUALITATIVE STUDY (STUDY 2)}

\section{Introduction}

This chapter presents a qualitative study that investigated the nature and experience of perceived discrimination (PD) in the workplace from the immigrants' perspective. The study aimed to explore the conceptualization of PD from a target's perspective by exploring the basic experiences of immigrants and investigating the prevalence of discriminatory experiences in the workplace context. This study was also conducted to gather information that will be useful in the construction of a measure of PD in the workplace. In relation to the proposed research framework (see Figure 3.1 of Chapter 3), this chapter addresses the middle portion of research framework introduced in the previous chapter.

As an overview prior to the presentation of the study results, the introduction section will cover changing trends in workplace discrimination, global immigration and New Zealand immigration. Local immigration facts and figures, immigration policies, and selected national demographics are presented to provide a background on the current policies that are being implemented on a macro level which could have an effect on the meso level such as work organizations. The methods section subsequently presents a qualitative exploratory study conducted to investigate the emerging workplace perceptions of immigrants and analysis of their perceptions as reflections of their work 
experiences. These perceptions will provide a basis for assuming that discrimination still exists in the workplace settings as evidenced by growing studies related to New Zealand migrant settlement outcomes.

The meta-analysis in Chapter 2 and the review in Chapter 3 identified the paucity of research on perceived discrimination particularly in the workplace context. This was deemed important and a pressing concern especially in this age of globalization. Increasing globalization continues to affect societies and brings unprecedented changes in global economy and migration trends. For instance, transnational interaction (communication beyond country boarders) is now facilitated through face to face or electronic communication because of increasing advancement in technology and dynamic economic activities worldwide (Aycan, 2000). As one aspect of the larger society, the workplace is also experiencing extraordinary changes in terms of workforce dynamics due to increasing ethnic diversity brought as consequences of immigration and emigration. Thus, Aycan (2000) points out the high importance of giving enough attention to understanding and improving conditions of work and the important role that organizational psychology plays in this process.

\section{Workplace Discrimination in the Context of Globalization}

Workplace discrimination research is mostly documented in countries that have histories of massive immigration and are now confronted with problems related to inter-group relations. Countries in North America and Europe have a long history of immigrant entry resulting in a high ratio of mixed races or ethnic groups. Consequent effects of these interracial and/or interethnic interactions, 
therefore, have been a source of interest by an increasing number of scholars (e.g. Blackaby, 2005, Leslie, Murphy, \& O'Leary, 2005; Cardo, 1994; Tolbert-Coombs \& King, 2005; Waters \& Jimenez, 2005).

Increasing studies are also being conducted in non-traditional receiving countries in Asia (see Lee, 1998; Shaffer et al., 2000; Foley, 2005) and the Oceania Region. Some Asian countries are experiencing increasing diversity in the workplace and in the society in general. For instance, China, Japan and Singapore are experiencing a high influx of migrants from neighbouring countries and expatriates from more developed countries. They are comparatively better off economically than their other Asian neighbours which make them lucrative work destinations for overseas temporary workers. These countries have a history of inflow and outflow of migrants mostly from less developed countries.

Lastly, scholars have recently embarked on discrimination research in countries that are considered as recent destinations of non-British immigrants such as the Oceania Region which includes Australia and New Zealand (e.g. Bennington \& Wein, 2000, Coates \& Carr, 2005; McDonald, Vechi, Bowman \& Sanson-Fisher, 1996, Pernice \& Brook, 1996; Ward \& Masgoret, 2007).

\section{Global Immigration: Changing Trends}

Large scale immigration, as a key effect of ongoing globalization, is unfolding its full impact for developed and developing countries in major parts of the world (Suarez-Orozco, 2005). It has been shown that the consequences of discrimination are as perennial as the continuing movements of people around the globe. Discrimination research follows recurring issues from old migration 
destinations such as Europe and North America to the new non-traditional receiving countries such as New Zealand. The economic and psycho-social consequences of migration continue to be of central interest in scholarly research. Thus, increasing studies are looking into changing behavioural and economic patterns brought about by movements of people.

Population shifts have been a global phenomenon since the beginning of time (Cohen, 1996). Historically, human migration was prompted by reasons of survival, adaptation and growth as exemplified by the hunters and gatherers who ventured across the globe, followed by colonizers in quest for power and wealth, and displacement of peoples who are fleeing from war-ravaged regions of the world (Marsella \& Ring, 2003). The recent migrant flows in the twenty first century are due to business, pleasure, education or search for greater well-being as facilitated by global economic integration, cheap communication costs and persisting economic gaps in rich vs. poor countries (Longhi, Nijkamp \& Poot, 2006).

The original reasons for migration translate to the present time as economic stability and pursuit for better standard of living. Winter-Ebner (1994) stated that relocations are mostly influenced by economic factors. Hence, economic prospects become a major decision point for moving to a new place (Ward et al., 2001). For instance, the home country lacks employment opportunities that match the qualifications and/or financial expectations of its labour force. People are henceforth motivated to move because of wage differentials and labour market failures back in their home country (Massey, 
Durand, \& Malone, 2005). Many educated and skilled citizens search for more lucrative job opportunities abroad as a consequence thereof. For the skilled migrant, it is a major and sometimes desperate decision to leave one's home country to search for the proverbial 'greener pastures'. But this decision is always coupled with the hope that one would get higher paying jobs and experience better working or living conditions.

An important development in latter part of the last century though is the intense market competition for skilled individuals such that economies can no longer depend on their local labour markets (IOM, 2005). Hence, accepting talented and qualified migrants bridges the skills shortage gap as well as contributes to the economy and labour market of the host country (Ho, Lidgard, Bedford \& Spoonley, 1997).

In addition, Suarez-Orozco (2005) described another characteristic of modern migration as being less confined within boundaries such that migrants are now traveling to and fro. Similarly, the International Organization for Migration (2005) reports that skilled migration is no longer unidirectional which means that countries can now gain from "brain circulation" (p.173) wherein they can take advantage from international sharing of highly educated and/or skilled individuals. Jackson, Carr, Edwards, Thorn, Allfree, Hooks et al.(2005) refers to the same concept as talent flow, which is the free flow of skills from one location to the next depending on how lucrative the offers are. Hence, countries that lose their local human resources to overseas competitors can in turn benefit from incoming skilled migrants. 
According to the latest worldwide census report, migrants are estimated to be at 191 million with an average growth of $1.5 \%$ over the last five years (United Nations [UN], 2006). This is an increase of 15 million compared to the 176 million from the year 2000 census making the migrant population, if situated in a single country, the world's fifth most populated nation (IOM, 2005). Overall, the migrant population is now $3 \%$ of the world's population with $60.5 \%$ of the migrants situated in more developed regions like Europe, Northern America, Japan, Australia, New Zealand (UN, 2006). According to this report, migrants comprise $8.8 \%$ of Europe's total population, $13.5 \%$ of Northern America's, $1.6 \%$ of Japan's, $20.3 \%$ of Australia's and $15.9 \%$ of New Zealand's. Most of these regions have an increasing trend of migrant flows.

In 2005, Oceania ranked as the region with highest immigrant population wherein $15.2 \%$ of its populace is composed of migrants (IOM, 2005). Most of the migrants are situated in two of Oceana's largest countries - Australia and New Zealand. These two countries have also been part of the traditional countries of destination for immigration (primarily from the British Isles) similar to the United States and Canada. New Zealand compared to Australia, Canada and the United States, is smaller in terms of size and economies of scale. However, New Zealand has already ranked second to Australia as having the world's highest percentage of immigrants in the workplace in 2004 (Phillips, 2008). Surprisingly, New Zealand has a more recent history of changes in terms of immigration laws. Due to its growing economy, there had been increased demand for skilled workers resulting from labour shortages that could not be satisfied by the local manpower 
pool (New Zealand Department of Labour, 2008). Thus, it is interesting to focus on New Zealand as a new destination chosen by migrants (mostly highly skilled) to live in and work, although it is situated in the southernmost part of the Oceania Region - almost at the edge of the world.

One of the continuing and pressing matters in the workplace and in the society in general is the issue of discrimination. Prejudice, discrimination and racism continue to be observed and experienced by the targets despite individual, societal and legal pressures (Dovidio \& Gaertner, 1986). The Human Rights Commission of New Zealand (2007) similarly reports increasing statistics of human rights violation complaints which are race related. It is to be noted that New Zealand officially abides by the Universal Declaration of Employee Rights wherein employers are directed not to discriminate in hiring and terminating workers due to race, colour, national or ethnic origin, sex or sexual orientation, marital or family status, employment status, age, religious belief or political opinion (Department of Labour, 2007). However, research data in the country reflect a conflicting story based on differing claims which will be discussed in the succeeding sections.

More importantly, another relevant issue that appears to be neglected is looking into workplace issues of immigrant workers who are the major contributor of increasing ethnic diversity in organizations affected by globalization. Employment difficulties were found to be a part of immigrant settlement. The psychological outcomes of skilled immigrants is thus of 
increasing interest simultaneous with existing problems related to social interactions in the workplace.

\section{New Zealand Immigration: Past and Current Trends}

New Zealand's population, akin to Australia, has historically been composed mainly of immigrants (Brosnan, 1988). The countries of origin of these immigrants were diverse depending on their motivations for migration such as economic factors, refugee status or colonial relationships with the nearby Pacific Islands. Currently, official bicultural relations between Maori (the indigenous inhabitants of New Zealand) and the descendants of the British settlers dominate over the other ethnic communities in the country. Government policies are directed towards acknowledging the rights of these two existing majority cultures and assisting further the settlement of minority cultures.

\section{History of New Zealand Immigration}

However, the history records would describe a different scenario regarding the past intercultural relations in New Zealand. Maori tried to defend their rights as original settlers versus the British colonizers during the 19th century which culminated in the signing of the Treaty of Waitangi in the 1840 s. The English version of the treaty (Waitangi Tribunal, 2009) referred to the British, Australians and European immigrants to be accorded residential rights as part of the Queen of England's sovereignty in New Zealand. Thus, only people from the said regions were acknowledged as immigrants and non-white peoples were definitely not accepted (McKinnon, 1996). This era marked the so called 
"Great White Walls" of New Zealand meaning that "white-skinned" people of selected ancestry are the only ones allowed entry to the shores of New Zealand.

Chinese peoples were recruited as mine workers in the southern part of the country as early as 1866 . However they were subjected to severe immigration restrictions including imposition of the poll tax. The poll tax was only abolished in 1944 after being implemented for half a century (Murphy, 2002). Subsequently, Pacific peoples became the first wave of 'visible immigrants' (migrants who are not British-descent) due to the political strife in the neighbouring islands of New Zealand. This influx addressed the country's need for semi-skilled and unskilled labour force during the 1950 ' to the 1970 's.

In 1986, a major policy review marked a significant change in the immigration laws of New Zealand. Thus the Immigration Act of 1987 paved the way for the active acceptance of immigrants regardless of race or ethnicity thus opening up the opportunity for those of non-European descent to come and live in the country (Ip, 2003). The growing economy of New Zealand has resulted in increased demand for skilled workers that could not be satisfied by its internal labour pool. Introduction of the Skilled Migrant Category provided a venue for the acceptance of migrants based on their educational qualifications and work experience that match the criteria in either the short-term or long-term skills shortage list. Thereafter, the succeeding years marked the increase of skilled and professional immigrants from non-traditional sources like Asia (Trlin et al., 1999) and the statistics have been increasing over the years. 


\section{Total Population}

The most recent census conducted by Statistics New Zealand (2006b) shows that Europeans (67.6\%) still make up the dominant ethnic group followed by Maori (14.6\%). Following as the third and fourth largest groups are Asians (9.2\%) and Pacific Peoples (6.9\%) respectively. Among these major groups, Asians had the highest increase whose population almost doubled from 2001 to 2006 followed by the Pacific peoples with a 14.\% increase over the last five years. (See Table 4.1 for the tabulation).

The European ethnic group totaling 2.6 million is composed of five specific ethnic groups, namely New Zealand-European, English, Dutch, British and Australian. The recent census reflect a decrease in number of New Zealand Europeans (11.7\% drop) due to the introduction of "New Zealander" category which was considered in the 2001 census as part of New Zealand -European but was changed in the 2006 census as part of the "Other Ethnic" category (for New Zealanders whose ethnicities are not found in the other categories).

Table 4.1

Distribution of New Zealand Population

\begin{tabular}{lcccc}
\hline \multicolumn{1}{c}{ Ethnic Groups } & $\begin{array}{c}\text { \% Total } \\
\text { Population } \\
\text { 2006 }\end{array}$ & $\begin{array}{c}\text { \% Increase } \\
\text { 5-yr period }\end{array}$ & $\mathbf{2 0 0 6}$ & \multirow{2}{2001}{} \\
& 67.6 & $-11.7 \%$ & $2,609,598$ & $2,868,009$ \\
\hline European & 14.6 & $7.4 \%$ & 565,329 & 526,281 \\
Māori & 6.9 & $14 \%$ & 265,974 & 231,801 \\
Pacific Peoples & 9.2 & $50 \%$ & 354,549 & 237,459 \\
Asian & 0.9 & & & \\
Middle Eastern/Latin & & & 34,746 & \\
American/African & 11.1 & & 429,429 & \\
Other Ethnicity & 0 & & 1,494 & 24,924 \\
- New Zealander & & & \\
- Other Ethnicity-other & & & & \\
& & & &
\end{tabular}

Source: Statistics New Zealand (2001/2006) 
The Maori ethnic group, composed of more than half a million, increased by $7.4 \%$ for the last five years reflecting a majority of the population belonging to the young generation (one-third of the population is aged under 15 years). This fact is being capitalized by the Maori as their major advantage over the aging population of the European ethnic group.

A dramatic increase in population occurred among the Asian ethnic group wherein the population increased by almost $50 \%$. Within this ethnic group, Chinese remain as the biggest group followed in a descending order by Indians, Koreans, Filipinos, Japanese, Sri Lankan and Cambodians. However, the Indian group has the highest percentage increase in population (68.2\%) for the last 5year period, followed by Koreans (61.8\%), Filipinos (52.7\%) and Chinese $(40.5 \%)$.

\section{Immigrant Population}

Historically, the United Kingdom (UK) and Ireland are the main source of people born overseas in New Zealand. However, the British/Irish population dropped from $32.2 \%$ (2001) to $28.6 \%$ (2006). This is in contrast to the trend for Asian-born immigrants that increased from $23.7 \%$ (2001) to $28.6 \%$ (2006). In effect, the proportions of immigrants born in UK/Ireland and Asia are now at par with each other. Overall, the population of the overseas-born has been on an increasing trend (see Figure 4.1). 
Figure 4.1

Migrant Population as a Percentage of Total Population

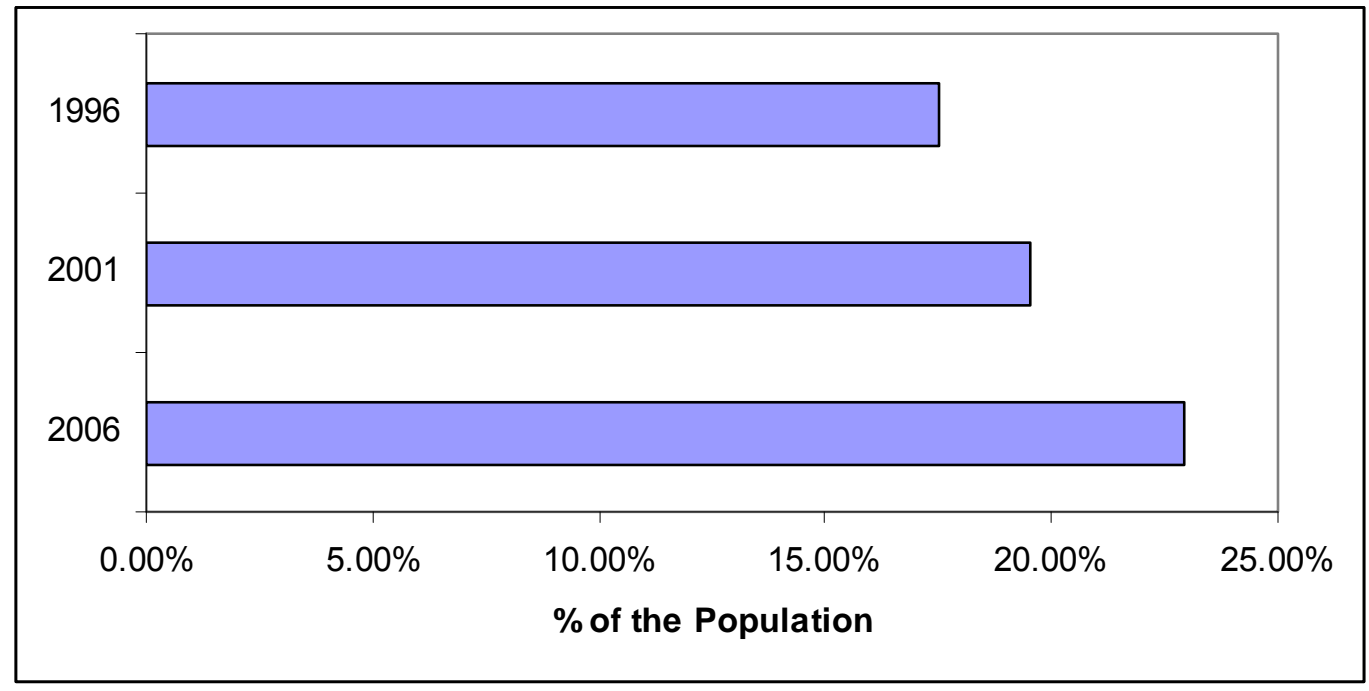

The significant increases in the immigrant population (see Table 4.2) were coming from migrants born in the People Republic of China and India whose population in New Zealand doubled during the last 5-year period, $100.6 \%$ and $107.5 \%$ respectively. Marked increases were also observed for the number of migrants coming from Korea, (60.6\%), South Africa (59.9\%) and Fiji (46.7\%).

\section{Table 4.2}

Percentage Increase in the Population of People Born Overseas

\begin{tabular}{lc}
\hline \multicolumn{1}{c}{ Country of Birth } & $\begin{array}{c}\mathbf{5 - y r} \\
\text { Increase }\end{array}$ \\
& $\mathbf{( \% )}$ \\
\hline England & 13.6 \\
People's Republic of China & 100.6 \\
Australia & 11.5 \\
Samoa & 7.5 \\
India & 107.5 \\
South Africa & 59.9 \\
Fiji & 46.7 \\
Scotland & 1.2 \\
Republic of Korea & 60.6 \\
Source: Statistics New Zealand (2006)
\end{tabular}




\section{Emerging Barriers to the Successful Immigrant Settlement in New Zealand}

Retrospectively, the changes in the immigration policies were largely due to the sustained efforts to meet the demands of labour shortages as a function of its growing economy (Department of Labour, 2005/2006). A focal point of New Zealand's current immigration policy is accepting skilled immigrants on the basis of the employability and transferability of skills demonstrated in having interested employers and qualifications that match the local requirements specifically in the skill-shortage list (Department of Labour, 2005). As reflected in their National Immigration Settlement Strategy from 2003, immigrants should be able to obtain jobs related to their qualifications and are encouraged to participate in the New Zealand society without giving up their own culture. As New Zealand society continues to be multi-cultural due the influx of immigrants, increasing workplace diversity would be a consequence since employment will be among the priorities of the incoming immigrants.

However, just like new settlers of other countries, incoming immigrants face workplace challenges during their period of re-settlement. A review of the employment outcomes of migrants is thereby an important step to investigate the barriers that hinder the successful settlement of immigrants especially in their work life. The common indicators used by the government and economic researchers to determine the success or failures of migration are economic measures like socio-economic status, rates of employment/unemployment and underemployment. 
Socio-economic status has been cited as one of the standard measures of immigrant integration into the host culture (Waters \& Jimenez, 2005). It is characterized by parity in earnings, occupational specialization and educational attainment. A study has linked income disparity to discrimination such that minority groups that report being victims of discrimination have incomes lower than host nationals (Herring et al., 1998).

In the local context, the latest census show that income levels vary according to the ethnic background. The "Other Ethnicity" and "European" categories ranked as the two highest in median annual income with $\$ 31,200$ and $\$ 25,400$ respectively. The lowest income earners are Asians with $\$ 14,500$ as their median annual income. This was explained by the Statistics New Zealand (2006) as probably due to a high proportion of Asians belonging to the youth bracket who were likely to earn less. Further, $58 \%$ of Asians earn less than $\$ 20,000$ per annum, the lowest of all ethnic groups (see Figure 4.2).

In terms of gender differences, the 2006 census show that women have lower median annual personal income compared to men $(\$ 19,100$ versus $\$ 31,500$, respectively). Income from employment is associated with women being less likely to be in paid work, in full time work, and working longer hours which could explain the disparity between the gender differences in income.

A more detailed description can be obtained in the 2001 census data showing the same income disparity between people born in New Zealand and those born overseas. Even more than 5 years ago, Asians were already earning less (ranging from $\$ 6,800$ to $\$ 13,900$ ) than all the groups with the exception of 
the migrants coming from North Africa and the Middle East $(\$ 10,000)$. Overall, migrants were earning less $(\$ 16,000)$ than their NEW ZEALAND-born counterparts $(\$ 19,200)$

Figure 4.2

Annual Median Income (NZ\$) of Different Ethnic Groups

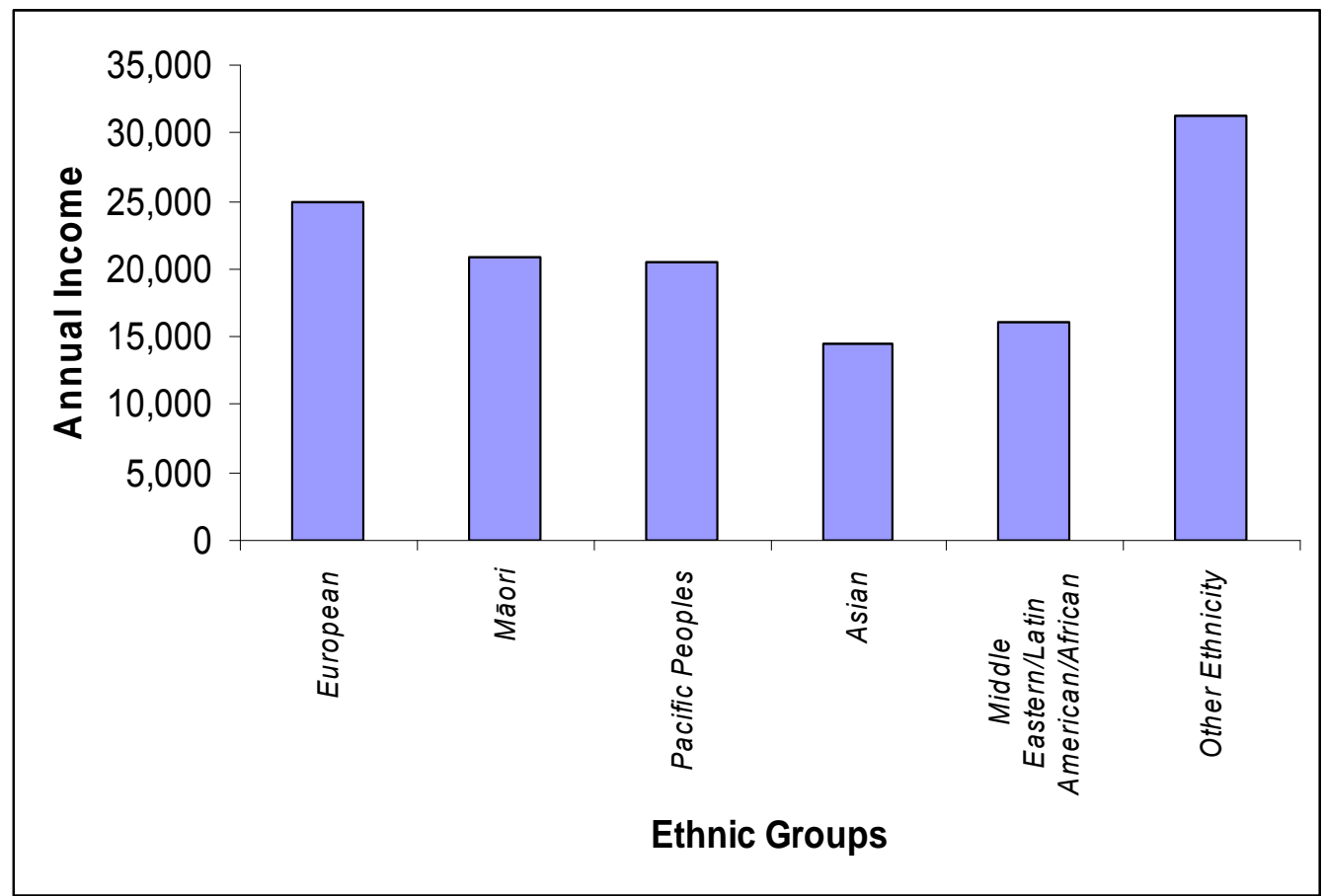

based on the annual median income (see Figure 4.3). Hence, the explanation of Statistics New Zealand for the Asians' income disparity in 2006 may not be due only to having a majority of young population.

Moreover, an earlier survey conducted by Statistics New Zealand (2002) showed that overseas-born $(9 \%)$ were more likely to be unemployed compared to local born New Zealanders (7.1\%) although the former group held higher 
Figure 4.3

Distribution of the NEW ZEALAND Population by Income (2001)

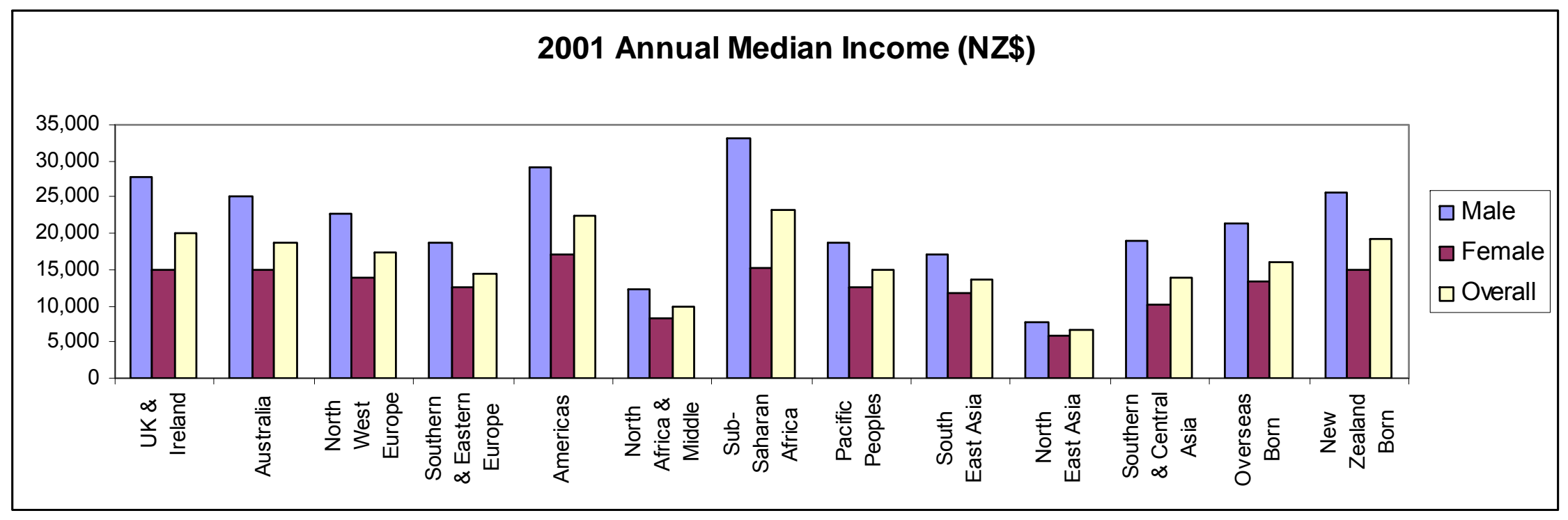

Source: Statistics New Zealand (2001) 
educational qualifications. The survey also reflected that highest unemployment rates were observed among North African and Middle Eastern peoples (23.8\%), followed by Asians (from 11\%-16.5\% depending on the Asian region) and the Pacific peoples (13.5\%). The lowest rates were reported for immigrants from UK and Ireland (4.2\%) and Northwest Europe (5.1\%). The unemployment rates of immigrants decreases according to the years of stay in the host country from $22.6 \%$ in the first year, $13.3 \%$ a year after, and $10.5 \%$ between $5-9$ years (Bedford, Ho \& Lidgard, 2001; Podsiadlowski \& Ward, forthcoming).

Specific to the immigrant population, a survey was conducted by the New Zealand Department of Labour (2005) using a sample of 1759 migrants of 64 different nationalities that were further divided into regions of origin, namely UK/Ireland, Europe/South Africa/North America (ESANA), Asia and Others. Those who were assigned to the region "Others" were those from the Middle East Countries, African countries, South American countries and the Pacific Islands. In this study it was shown that almost all of the respondents who were accepted in the Skilled Migrant Category were employed, i.e. UK/Ireland (98\%), ESANA (96\%), Asia (98\%) and Other (100\%). Tabulated in Table 4.3 are: 1) the distribution of participants from the different regions; 2) distribution of those who were accepted in the Skilled Migrant Category (SMC); and 3) percentages of professionals in the sample. 
Table 4.3

Survey Participants according to Region of Origin, SMC \& Professionals

\begin{tabular}{|c|c|c|c|}
\hline Region & $\mathbf{N}$ & SMC & Professionals \\
\hline UK/Ireland & 577 & 322 & $29 \%$ \\
\hline ESANA & 224 & 123 & $30 \%$ \\
\hline Asia & 225 & 96 & $34 \%$ \\
\hline Other & 86 & 47 & $26 \%$ \\
\hline
\end{tabular}

Note: SMC (Skilled Migrant Category)

The majority of the participants $(85 \%)$ reported that it was easy for them to obtain jobs upon gaining residency. The remaining $15 \%$ who experienced difficulty reported 1) lack of New Zealand work experience, 2) language difficulties, 3) skills or experience not accepted by local employers and 4) no suitable work was commensurate to their level of qualifications as the top four reasons for their difficulty.

Most, if not all, of those who apply under the Skilled Migrant Category have at least a bachelor's degree to get appropriate points for that criterion. However, when migrants in the survey reached the country, 52\% of Asians and $56 \%$ of "Others", $39 \%$ of UK/Ireland and $42 \%$ of ESANA had to take up further education and training citing various reasons. Upgrading their qualifications was common as a response among the four regions. Migrants from UK/Ireland and ESANA took up further studies as leisure activity or personal interest. Asians, on the other hand, mainly do it to improve English language skills. Lastly, Asians and Others both take up further studies to get a job/better job as their second highest response. 
Asians had the highest proportion of migrants working as Professionals but despite this the survey shows that they have the lowest average income. As illustrated in Figure 4.4, 65\% from UK/Ireland, 52\% from ESANA, 24\% from Other and 19\% from Asia are earning above the income bracket of $\$ 50,001$ (from the main job). On the other hand, $60 \%$ of Asians, 37\% Others, $25 \%$ ESANA and $15 \%$ from UK/Ireland are earning below $\$ 40,000$.

\section{Figure 4.4}

Income Distribution of Survey Participants

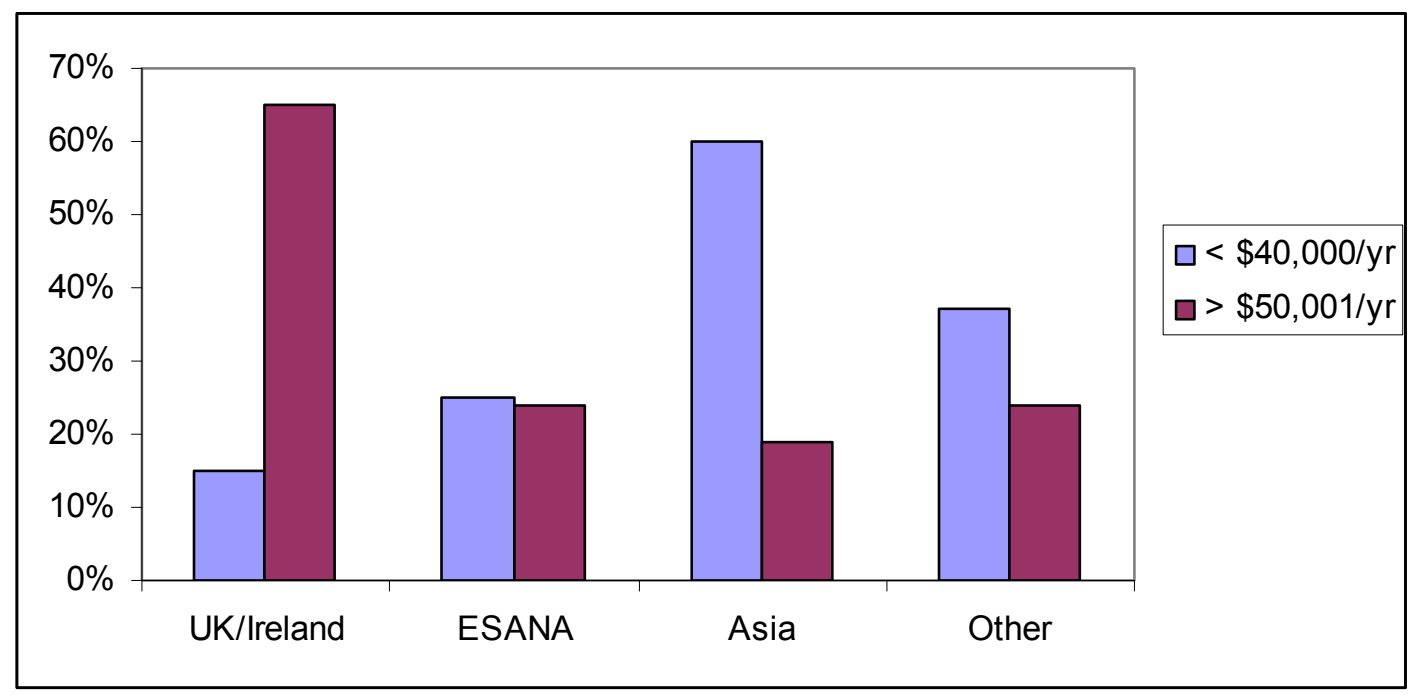

Overall, it appears that the immigration system of New Zealand has come a long way from being exclusive (mostly European migrants with preferential treatment to the British descendants) to being inclusive of migrants coming from different ethnic and cultural backgrounds (as exemplified by the huge entry of migrants from non-traditional sources such as Asia). It should be noted, though, that there are skills criteria that have to be met prior to being accepted in the 
country in response to the growing skills shortage list. Upon settlement in the country, employment outcomes of migrants show differences (i.e. with more positive outcomes to Europeans compared to Asians) which could be attributed to several factors such as the environmental climate as dictated by government policies, inherent organizational make-up and attitudinal differences. Specific aspects are worth investigating for a deeper understanding about the issues at hand.

\section{Are New Zealand Workplaces Discriminatory?}

A growing number of qualitative and quantitative empirical studies in New Zealand show that immigrants are subjected to unfair practices that are attributed to local authorities and employers as part of the regular employment policies or qualifications accreditation procedures. Initially, skilled immigrants face difficulties in practicing their professions due to the non-recognition of their qualifications obtained overseas. A specific example comes from a study conducted with physician-migrant professionals who were hindered to practice their medical profession due to difficulties in obtaining recognition of their overseas qualifications from the New Zealand Qualifications Authority or NZQA (Selvarajah, 2004).

Another indication of subtle forms of discriminatory practices by New Zealand employers and recruitment agencies has been identified in connection with the preferential selection for job applicants from Europe, South Africa and North America (ESANA) and those with English-sounding names thereby discriminating against other migrant groups specifically Asians applicants 
regardless if qualifications are equivalent (Coates \& Carr, 2005; Stewart, 2005; Ward \& Masgoret, 2007; Wilson et al., 2005).

Immigrants, therefore, perceived their personal characteristics as barriers to successful employment outcomes. For instance, migrant respondents cited ethnic identification, colour of the skin, accent and having a foreign-sounding name as reasons for the perceptions of being discriminated during preemployment and employment stage (Basnayake, 1999). Further, the New Zealand State Service Commission (2002) reported that employees in the public sector cited gender closely followed by ethnicity as the two top most grounds for discrimination.

Another migration research conducted by Butcher, Spoonley \& Trlin (2006) reflected the immigrants' sentiments of being discriminated and socially excluded. The authors conducted focus groups discussion in the three largest cities of New Zealand (namely Auckland, Wellington and Christchurch) and found that the respondents expressed difficulties in finding jobs due to their accent, qualifications not being recognized, New Zealand local work experience is required and cost for certifying overseas qualifications are very high (e.g. $\$ 5000$ for medical degree). The respondents also felt being discriminated in terms of access in goods and services like housing and education. The study aptly reflects the participants' feelings of being "under-valued, under-utilized and ignored".

Resulting outcomes to these perceptions often lead to negative consequences. As early as 1983, prior to the significant change in the Immigration Act, Marsh and McDonald (1983) reported on the difficulties faced by 
Polynesians in interacting with a multi-cultural workforce within the manufacturing industry.

In addition, a qualitative research by Trlin, Henderson and North (1999) reflected how unemployment affects negatively the adaptation outlook of 36 Indian participants who came to New Zealand under the General Skills Category. Consequently, unemployment and underemployment have been found to be risk factors that affect Asian's mental health but only during the first two years of the immigrants' arrival at New Zealand (New Asian and Migrant Health Research Centre, 2004).

A longitudinal study linking employment and mental health of three groups of immigrants (Chinese, Indians and South Africans) showed that low levels of mental health were evident whether the participants were employed or unemployed and regardless of their national background (Pernice, Trlin, Henderson, \& North, 2000). The lack of significant differences was explained as even though participants were employed, most expressed that they were "underemployed" and experiencing work stress. Furthermore, South Africans who were hypothesized to have higher level of mental health than the Chinese and Indian participants, demonstrated similar low levels of mental health. This is attributed to the reason that South Africans are considered as "semi-voluntary immigrants to New Zealand since motivation for migration was a mix of push (i.e. violence, crime) and pull factors (improved quality of life) unlike British immigrants who come to the country mostly due to pull factors. 
Lastly, a recent literature review by Podsiadlowski (2006) showed that social inequality and discrimination were evident in the demographic data obtained from the 38 studies that looked into immigration trends in New Zealand from 1998 up to 2006. The results reflected income gaps and employment outcome disparity for minority groups as a function of their ethnic backgrounds and gender. Podsiadlowski's (2006) workplace-based study provided proof that up to the present the 2003 National Immigration Settlement Strategy has yet to be achieved by policymaking bodies of New Zealand. The figures found in this study are consistent with the census study on migrant income wherein some ethnic migrant groups (e.g. Asians) experience lower economic outcomes (Statistics New Zealand, 2006b) though the majority of the respondents occupy professional positions (Department of Labour, 2005).

Contrary to the above-mentioned studies and surveys, recent reports by the New Zealands Department of Labour $(2005,2006)$ show that $78 \%$ out of 1759 (in 2005) and 79\% of the 2060 (in 2006) skilled migrants participants answered that they were "very satisfied/satisfied" with their job. For those who responded that they were "dissatisfied" ( $8 \%$ on the average for two years), the reasons given were low pay, non-utilization of skills and experience, not obtaining desired occupation and hoping to work different or more hours. These latter reasons were congruent with the definition of the Labour Utilization Framework of the three dimensions of underemployment, namely hours of work, income from work, and skill mismatch (Friedland \& Price, 2003). This figure, albeit minimal, shows some form of evidence of immigrants being underemployed in the New Zealand 
workplace. Furthermore, as stated in the Social Cohesion Report (2007), discrimination is unlikely to be accurately reported by migrants probably because of lack of knowledge of the policy and procedures for filing a complaint, language barriers and fear of losing their right to stay in the host country. Unfortunately, research shows that even the number of grievance reports in the workplace does not predict the extent of employees' perceptions of discrimination (Ensher, 2001). Hence, the level of perceived discrimination could be higher than what is currently being reported.

The aforementioned studies thereby present two different scenarios regarding discriminatory experiences and perceptions that immigrants are facing in New Zealand as a function of their ethnic and cultural backgrounds confounded by other demographic characteristics such as gender age and language differences. The immigrants' negative experiences are mostly opposite to their rosy expectations prior to migration.

Although it seems that immigration problems and discrimination that appeared in the studies of early migrations in Northern America and Europe continue to persist even in new destinations like New Zealand, there are official government reports that present a more positive outlook for migrants in New Zealand. These reports are usually measured by demographic data and single items measuring satisfaction and well-being (see Department of Labour, 2005). Thus, a more comprehensive scale measuring the demographic and psychosocial outcomes of migrants can assist in the deeper understanding of the plight of 
migrants in New Zealand workplaces. These gaps will be addressed by this current study.

\section{Study 2:}

\section{Focus Group Discussions}

\section{Specific Objectives}

Due to the differing claims regarding the predicament of immigrants in New Zealand, the author embarked on an exploratory study regarding the emerging perceptions of New Zealand immigrants in their workplaces. Hence, this study was conducted to serve as a preliminary investigation of the prevalence of discrimination based on immigrants' workplace experiences in New Zealand. Specifically, the first aim of the study was to look into the workplace experiences of first generation immigrants who are employed in various New Zealand organizations. By gathering their experiences (positive and negative) related to their employment history including pre-employment experiences up to their current job assignments, we could draw a clearer picture of how well (or badly) these skilled immigrants are adapting in their current work environment. The second aim of this study was to gather concepts that can be included in the construction of a scale that will be used to measure employee perceptions of discrimination. The scale is aimed at capturing the salient workplace experiences or perceptions of employees like immigrants in the New Zealand work context. These concepts will be compared with the existing literature on workplace discrimination.

Due to its exploratory nature, no hypothesis was initially posed in designing the study. Instead, a main research question was posed which guided 
the design of the succeeding minor research questions. The main research question was what are the emerging perceptions of discrimination (PD) for migrants in their New Zealand workplaces as exemplified in their workplace experiences? From these workplace experiences, the following specific research questions were also posed: (a) How do immigrants perceive the New Zealand workplace? (b) What experiences are common to immigrants? (c) What experiences are specific to certain groups? (d) What can be concluded out of these perceptions?

\section{Focus Groups as a Research Method}

Focus Groups Discussion (FGD) was considered an appropriate method in gathering data for this study because the spontaneous interactive discussion or conversation among a group of people can lead to the generation of ideas, thoughts or emotions that may not be possible in individual interviews (Hair, Bush, \& Ortinau, 2006). Groups were homogeneous in terms of countries of origin to eliminate hesitation of sharing of ideas due to cultural differences. Further, maintaining homogenous groups facilitated validation of experiences within a specific ethnic group.

\section{Method}

\section{Participants}

The focus groups discussions (FGD) sample was composed of firstgeneration immigrants working in Wellington City. They were grouped according 
to the country of origin, namely China, India, Philippines, Germany, and Zimbabwe. First generation is defined as immigrants born outside of New Zealand. Immigrants were chosen rather than sojourners or other temporary workers because immigrants have long-term intentions to stay in a new country thus earning them permanent resident legal statuses.

Representatives from five countries (as their birthplace) were chosen for the following reasons: a) English is not their first language; b) The Asian group is the largest group of immigrants that came into New Zealand in the last two decades (Philipps, 2008). Thus, getting representatives from this group was deemed important in contributing to the examination of Immigrants' workplace experiences in New Zealand. This group was also considered as the representative of the "visible" minority in a previously predominantly White-dominated culture; c) Among the Asians, the Chinese group is the largest ethnic group. Chinese peoples arrived in New Zealand in the 1870's as workers in the gold mines of in the southern part of the country. However, only lately (1980's) that Chinese were granted legal immigration status (Philips, 2008); d) The Indian group, the second largest Asian group, has the highest population increase within the 5-year period. Thus, Indians comprise a large proportion of the Asian group in New Zealand; e) The Filipino group is the fourth largest Asian ethnic group next to Koreans, but it was considered more practicable to involve the Filipino group in the FGD because of their relative English competency than Koreans though English is second language for both groups. English is the second official language in the Philippines and it is part of the academic curriculum from primary school. Hence, 
Filipinos are expected to be well versed in English if they have completed their university education; f) the German group is a representative group of the "nonvisible" minorities in New Zealand. Although in the 2001 census Germans were only next to Dutch as European Groups with English as a second language, they were chosen because they also had a long migration history in New Zealand dating back to the 1860 's gold mines in the South Island similar to the Chinese (Philips, 2008); and g) the last group, the Zimbabweans were chosen because they are another representative of the "visible" minorities and are comparable to the African Americans who are widely researched as a highly discriminated racial group. In 2004, the highest African nationalities in New Zealand were those coming from South Africa, Somalia, Egypt, Zimbabwe and Ethiopia (Walrond, 2007). Somalians and Ethiopians came to the country primarily as refugees. Zimbabweans were eventually chosen for the FGD because of ease in recruitment. I got acquainted with a Zimbabwean working in the Wellington City Council who became my contact person and helped in recruiting Zimbabweans who came to New Zealand as skilled immigrants.

Twenty-three immigrants participated in this study with 4-5 participants for each ethnic group representing the countries mentioned above. The respondents initially came to the country either with student visas $(26 \%)$, principal applicants $(48 \%)$ or secondary applicants $(26 \%)$ in the skilled migrants category. Those who came to the country as international students eventually obtained their work permits within New Zealand and applied as permanent residents under the skilled migrant category. The sample was composed of 13 
females and 10 males. Participants' ages fell in the range between 20-60 years with an average age of 41.5 years old. Total years of residence in New Zealand varied from eight months to 24 years with an average of seven years residency. All of the participants have earned their university degree from their home countries while $39 \%$ have pursued or were pursuing Masters or Doctorate Degrees (3 Chinese, 2 Filipinos, 2 Germans, 1 Indian, 1 Zimbabwean) in areas such as Finance, Management, Physics, or Accounting while employed. Chinese participant was a Doctor of Medicine in China but was not able to practice her profession in New Zealand.

Most of the participants were employed (83\%). Out of the four unemployed participants, two were involuntarily unemployed but actively looking for jobs, one was not actively looking and the last one was a retired employee. All unemployed participants had worked in New Zealand prior to their unemployment status. Out of the employed participants, the majority was working in the private sector (68\%), four in the public sector (21\%) and two (11\%) in the semi-private sector (e.g. academe). The respondents' occupations were information technology/programmers $(37 \%)$, analysts $(26 \%)$, admin work $(21 \%)$, manual work in the health sector (11\%) and accounting (5\%). Of those who were employed in the information technology (IT), 57\% pursued computerprogramming courses in NEW ZEALAND or had obtained IT work experience outside their home countries before coming to New Zealand (43\%). Table 4.4 shows the tabulated demographic characteristics of the FGD participants. 
Table 4.4

\section{Demographic Details of Focus Groups Discussion (FGD) Participants}

\begin{tabular}{|c|c|c|c|c|c|c|}
\hline & TOTAL & Chinese & Indians & Filipino & Germans & Zimbabweans \\
\hline No. of Participants & 23 & 5 & 5 & 5 & 4 & 4 \\
\hline Female & 13 & 5 & 1 & 4 & 3 & 0 \\
\hline \multicolumn{7}{|l|}{ Age Range } \\
\hline-20 to 30 & 2 & 1 & 1 & & & \\
\hline-31 to 40 & 8 & 3 & 2 & 1 & 1 & 1 \\
\hline-41 to 50 & 10 & 1 & 2 & 3 & 1 & 3 \\
\hline-51 to 60 & 2 & & & 1 & 1 & \\
\hline-60 and up & 1 & & & & 1 & \\
\hline $\begin{array}{l}\text { Highest Educational } \\
\text { Attainment }\end{array}$ & & $\begin{array}{l}\text { Doctor of } \\
\text { Medicine }\end{array}$ & $\begin{array}{c}\text { Master's } \\
\text { Degree } \\
\text { Level }\end{array}$ & $\begin{array}{l}\text { Masters' } \\
\text { Degree }\end{array}$ & PhD Level & $\begin{array}{l}\text { Master's } \\
\text { Degree }\end{array}$ \\
\hline \multicolumn{7}{|l|}{ Job Status } \\
\hline - Permanent & 19 & 3 & 5 & 5 & 2 & 4 \\
\hline - Unemployed & 4 & 2 & & & 2 & \\
\hline \multicolumn{7}{|l|}{ Current Occupation } \\
\hline - Admin & 4 & 1 & 1 & 1 & & 1 \\
\hline - Accounting & 1 & & & 1 & & \\
\hline - Analysts & 5 & & & & 2 & 3 \\
\hline - Information Technology & 7 & & 4 & 3 & & \\
\hline $\begin{array}{l}\text { - Manual/Labourer (Health } \\
\quad \text { Services) } \\
\text { Sector of the Organization }\end{array}$ & 2 & 2 & & & & \\
\hline - Private & 13 & 3 & 4 & 1 & 2 & 3 \\
\hline - Public & 4 & & & 3 & & 1 \\
\hline - Semi-Private & 2 & & 1 & 1 & & \\
\hline
\end{tabular}




\section{Materials}

A semi-structured discussion guideline with open-ended questions was used in the group discussion. It was deliberate that no reference to perceptions of discrimination or discrimination experiences was made in the guide questions so as not to pre-empt the responses of the participants. Below is the list of the guide questions used during the discussions.

1. Can you share your reasons for moving to New Zealand? [Probe - What were your expectations in terms of advantages and disadvantages in you're a) profession b)income, c) family life]

2. How did you apply for work in NZ?

- What happened during the application process?

3. In your workplace, can you please describe your

- job assignment or the task that your doing in comparison to your qualification

- methods of payment and rewards /benefits /training

4. In your workplace, can you please describe your relationships with

- Peers/immediate supervisors/higher superiors

5. Can you recall any experience that hindered/helped you in

- feeling at ease with your job/feeling at ease with your co-workers

6. How did these experiences affect your work and your commitment to the organization?

7. In which way your experiences at work affected your long-term plans?

8. Overall, how would you describe the way your organization deal with immigrants or people from different ethnicity?

\section{Procedure}

Aside from being $1^{\text {st }}$ generation immigrant, the other criteria for selecting immigrant participants included: a status of permanent resident (PR) or New Zealand citizen, being 21 years old and above, and having at least six months work experience in the New Zealand workplace. They were recruited through snowballing technique and through contact persons in ethnic 
associations and organizations in Wellington. There was a contact person for each ethnic group who facilitated the dissemination of the research information to the participants. A copy of the information sheet was given to the contact persons beforehand in case the prospective participant wanted to have prior information about their participation. The contact persons for the Indian and Filipino groups were allowed to sit in the sessions but they were requested to observe the same ground rules as the participants. Opinions or data shared by the contact persons were excluded in the analysis because they were considered only as observers. I acted as facilitator for each discussion group.

The focus groups discussions were conducted from April 2006 to May 2006. A total of seven qualitative small group discussions or sessions were conducted in a pre-arranged venue within the university (one each for the Germans, Filipino and Zimbabwean groups, two sessions for the Indian, and two sessions for the Chinese groups). Each session lasted to almost two hours. It was initially planned to conduct one discussion session for each nationality but the Indian and Chinese participants could not make it to the appointed time. The scheduled times for these groups had to be postponed a few times. Hence, for the Indian and Chinese groups, the first sessions were composed of three members and the second sessions were composed of two members. Their responses were combined as one group in the analysis.

Consent forms attached to the information sheets were signed by the participants prior to each session. Demographic data such as age, ethnicity, gender, country of birth, educational and professional backgrounds were gathered during the sessions. All discussions were conducted in English. 
English is the primary language in New Zealand and immigrants are required to pass the English proficiency requirement of the New Zealand Immigration Service. A particular level of English proficiency is also required for those who are undertaking studies in New Zealand and for those who apply for a work visa. The changes in the Immigration policy in 1995 and again in 2002 raised the English language requirements for entry (Phillips, 2008). Thus, immigrants especially those entering in the skilled migrant category should pass this requirement. The participants were given NZ $\$ 10$ grocery vouchers as a token of appreciation for their participation. The discussions were audiotaped and transcribed by the primary researcher. Participants' names and other identifying details mentioned in the audio tapes were coded in the transcriptions to ensure that the data were not directly traceable to them.

\section{Data Analysis}

The transcribed data from the focus groups discussions were analyzed manually through a qualitative research analysis tool called Thematic Analysis. Thematic Analysis (Braun \& Clarke, 2006) is defined as "a method for identifying, analyzing and reporting patterns (themes) within data” (p.79). Each theme consists of patterned responses that represent a significant aspect of the data in addressing the research question. These patterned responses do not rely solely on prevalence but also on the consistency of analysis by which the data was coded. Overall, the focus is on the relevance of the theme in answering the research question.

Braun and Clark (2006) further described Thematic Analysis as an analytical tool not bounded to any pre-existing theoretical structure thus it can 
work with most of the existing theoretical frameworks. In this study, Thematic Analysis was used as a contextualist method that Braun and Clarke (2006) defined as looking into how individuals try to create meanings out of their experience and at the same time acknowledging the influence of the societal context in shaping those meanings. Furthermore, the "themes" or patterns of responses were data driven since they were identified through the inductive approach (Patton, 1990). The inductive approach is characterized by obtaining generated themes that do not directly resemble the specific questions asked. The coding process had no initial coding guide and it was not influenced by any theoretical inclinations. The specific research questions eventually evolved through the process of coding. Finally, themes were identified on a semantic level (Boyatzis, 1998) wherein themes were mere surface descriptions of the coded data leading to interpretation in order to hypothesize the implications and significance that go beyond the pattern of responses (Patton, 1990). Based on the above method of analysis themes were generated for further analysis.

Specifically, the coding system in this study involved the following process, a) scanning through the transcripts and identifying significant statements; b) categorizing the significant statements into themes within each group, c) identifying themes that are specific to each group, and d) comparing themes that are common among groups.

The primary researcher and a second rater (another $\mathrm{PhD}$ student using qualitative techniques in her thesis) independently read all the transcripts and initially coded for two nationality/ethnic groups. Then they met and discussed the codings that they did separately and compared their outputs. During 
several discussions, differences in the coding were rectified. From thereon, the primary researcher proceeded with the coding of the whole dataset. After the researcher finished all the codings, the themes generated were presented to the the second rater and the thesis supervisor (there was a different supervisor at this stage of the thesis who also previously read all the transcripts). They verified the coding and the thematic structure and made additional suggestions. Since the second rater did not code for all groups and the discussions were informal, no related statistics (e.g. Cohen's kappa) was computed to test inter-rater agreeement.

\section{Results}

The transcriptions were analyzed using Thematic Analysis (Braun and Clarke, 2006) and the coding system generated the following themes, 1) Overview of the New Zealand organization; 2) Culture-Specific Barriers to Positive Employment Outcomes; 3) Culture-Specific Facilitators to Positive Employment Outcomes; 4) New Zealand -Specific Pre-Hiring Barriers to Positive Employment Outcomes; and 5) New Zealand -Specific On-the-job Barriers to Positive Employment Outcomes.

\section{Overview of the New Zealand Organizations}

The organizational structure was viewed to be in contrast to what they were used to back in their home countries. It was generally described as a "flat" structure wherein interactions with superiors were quite informal. The participants would call their managers by their first names. This was 
considered different from their experience back home of calling their bosses with titles such as Sir, Madam, Mister, Doctor, and the like.

As one Filipino respondent has shared:

In the insurance company that I am in, the structure is very flat. Very very flat. So we are about $50 \ldots$ under the clientele relations division.... and from $\mathrm{A}$ to $\mathrm{Z}$, we are all the same. Above us are three team leaders, as the team leaders work with the general managers, operations manager. And one thing that you realized is ah you can even talk informally to the general manager on first name basis. (Filipino5F).

Relationships with managers were also described by the participants as friendly and cordial. The same experiences were expressed for peers. There was no stated outright animosity among peers related to cultural differences. Nonetheless, even if there was the perception of informal relationships with superiors, it was mentioned that there was still a kind of a subtle hierarchy. The subordinates among the participants were careful about the things that they will ask or be aware of the things that were being discussed with the superiors.

Furthermore, the workplace was described by most participants as a relaxed working environment. It stemmed from having good working relationships with peers and superiors and also from the pace of how work was conducted. For most respondents, they would view the relaxed environment as positive although quite contrary to what they were used to back home. As one German participant articulated: 
If your job started at 8o'clock, you start at 8 o'clock. And you have been to the toilet, and you had your coffee and you started your job at 8 o'clock... And here, lots of people would then come "Hi, how are you, how was your weekend, how was grandma, how was the dog? Ok, I'll need to go and put my make-up on, and I have to have a cup of tea!" That is not the German way you know... And some Germans here could not cope with such a relaxed way. (German2F)

\section{Culture-Specific Barriers to Positive Employment Outcomes}

Each participating ethnicity discussed some salient experiences that were particular to their group. These experiences where viewed to be either a hindrance or an advantage in obtaining and maintaining a suitable job. A suitable job was described as an occupation that was appropriate and commensurate with their qualifications and work experience before coming to New Zealand.

The salient topic for the Chinese group was on communication skills. They talked about the necessity of having English proficiency in looking for jobs in New Zealand. Some Chinese respondents stated that they were at times rejected by some locals who gave "do not understanding them" as an excuse. They also felt that New Zealanders try to avoid talking to them or mentoring them at work because it will entail more effort to accomplish these with Chinese people than with others who have better English skills. As perceived by a Chinese respondent, New Zealanders felt more relaxed dealing with people having English as a mother tongue especially if they were trying to 
explore some topics for discussion or talking about cultural backgrounds. A Chinese participant provided a concrete statement as an example:

Language is a barrier to enter workforce... if you are Kiwi you really don't like second language people to work with you because...maybe they thought ah, they need spend time, more energy to guide you or something like that. Yeah. It's very hard to find a suitable job here. (Chinese4F)

The Filipino group talked more about their propensity to be humble as prescribed by their cultural background. This behavior was considered a laidback attitude wherein people coming from this ethnicity were viewed as not assertive even if they did possess some skills. Hence, those who were trying to look for jobs cannot assert themselves in the interview, which was considered a major requirement for being able to obtain a job. Selling yourself to the employers included presenting oneself, asserting the skills and capabilities. Filipino participants thought that they were not good at this because this was considered as bragging. In a culture where humility was inculcated, bragging was a behavior seriously frowned upon. Hence, they felt that in order to break the barriers to employment, Filipinos should learn how to assert themselves and be able to communicate their capabilities effectively. As illustrated in this quote:

We are not as assertive... it's cultural...But, we work quietly, we try to prove our worth in the long run. We rather prove our worth in the job rather than say we're good...that's how are. It's basically the culture thing...it's submissive, we've been under Spanish colonial rule. (Filipino5F) 
The Zimbabwean group was composed of dark-skinned participants. This is to clarify the composition of the group since there are also white Zimbabweans in New Zealand. The topic that was most salient for this group was the feeling of constantly proving one's self because of barriers that prevent them from reaching their full potentials. All of the participants were employed in line with their professions but they felt that they could be in higher position levels if not for their skin colour. Racism was described to be present though subtle in New Zealand as it is everywhere (i.e. Australia). It was something that they thought they could not overcome unless they start to collaborate with Islanders and Asians in New Zealand. Hence, they felt that they would succeed in numbers if other ethnicities not considered "white" would come together and cooperate with each other. A Zimbabwean participant who was comparing New Zealand and Australia in terms of prevalence of racism stated, "The racism is alive and kicking here. The racism [there] is no worse than here. The difference is we're getting better money" (Zimbabwean4M).

\section{Culture-Specific Facilitators to Positive Employment Outcomes}

Adherence to schedule, straightforwardness and commitment to the task were the salient topics for the German group. The participants' discussions centered on being able to integrate the German culture with that of the New Zealand's because if one tries to be rigid about one's own ideas and approaches, then a German would not be able to adapt to the New Zealand way of life. According to participants of this group, the German's work ethics was also found by New Zealand employers as advantageous in finishing tasks 
on time and confronting problematic business related issues. One participant mentioned her "Germaness" has helped her a lot in being a good performer. She relayed that the boss relied on her for tough assignments such as going after clients with outstanding debts with the company and she knew she will not be sent on "diplomatic missions". Furthermore, the participants felt that they were successful in New Zealand because they were able to be flexible between balancing their strict German work ethics with that of the relaxed working environment and relationship-building culture. Her thoughts were revealed by this specific statement:

I had a funny thing...I took over a large portfolio, millions and millions of dollars and it had late run and nothing has happened about it. So basically I came with the job and more than 3 months I pretty much completely reduced the whole debt....and my bosses said "Yes (nameG3) you're the Rottweiller. I'll just send you out and let things happen (smile)...I wasn't offended in the slightest...if you ask me to do something, I do it. It's done! (German3F).

Like the German group, the Indian participants considered themselves also as a more successful group if compared to their other Asian counterparts. They associated this success with the "Indian mentality" of being pragmatic. They said that they prioritize more on pursuing careers that may not be what they were originally passionate about, as long as this career can ensure their economic stability. After achieving financial success then they can start pursuing their desired careers. Four out of the five participants were into Information Technology (IT) because this profession was in the skills shortage 
list of New Zealand. Initially, they had tertiary qualifications in Mathematics or Bachelor in Science, which was described as a general course. Although they were already in IT now, some of them expressed pursuing different careers later on such as Marketing and establishing their own business ventures.

The fact that Indians have a sort of a mentality as well? Like you're doing a sort of a course that kind of assures you even if it has nothing to do with your interest and loves in life. But it has to do with something that will... you can survive one for the next 50 years of your life" (Indian5F).

\section{New Zealand-Specific Pre-Hiring Barriers to Positive Employment}

\section{Outcomes}

All groups expressed difficulty in finding a suitable job in New Zealand. The majority expressed their experiences of trying hard to look for jobs that were commensurate to their qualifications and previous work experiences gained overseas. However, after several months of job hunting and numerous curriculum vitae or resumes being sent to companies, they would usually get rejections for their applications citing various reasons. The factors that usually hinder immigrants to obtain their desired jobs were:

\section{No "Kiwi" experience.}

New Zealanders are also referred to as Kiwis. Thus, having no "Kiwi" experience meant that the individual has not gained any work experience in New Zealand. "Kiwi" experience or New Zealand work experience was considered discriminatory by a Zimbabwean respondent because it was a vague criterion for employment. There was no definite explanation what 
"Kiwi" experience was and what exactly the employers were looking for in that experience. However, there was another concern on how can you get a "Kiwi" experience if you cannot get a job in the first place. One of the Zimbabwean participants just interpreted this as “...just like the Kiwi experience that they talk about time and again. They say they want your experience. They're simply saying - Sorry you're a foreigner, you don't really belong here. This is reserved for us" (Zimbabwean1M).

Having a "Kiwi experience" or having been employed by another New Zealand employer was viewed to be a plus factor in facilitating entry to a workplace. Some participants would usually consider this as gaining work experience in whatever areas as long as the employment was in New Zealand. Hence, most participants would accept low level jobs or jobs totally irrelevant to their qualifications (e.g. a Masters degree graduates doing a cleaner's or janitorial job or cashiering jobs) just to get New Zealand work experience and hopefully gain entry to a much better employment later on. As one Zimbabwean with a postgraduate degree shared:

In terms of work, I did all sorts of work. As a general hand...I worked as a cleaner... and laundry attendant in a backpacker in Auckland...I got a job with... a bakery assistant with Foodtown \{chuckle\}. Ah, but I entered a management training program. But of course in various times I was doing the floor of the supermarket...I was quite happy because ah...it was winter yeah. $\{$ laughter\}. I was tending the oven $\{$ laughter\}(Zimbabwean1M) 


\section{Qualifications not recognized.}

Some participants have experienced that their qualifications were not recognized by institutions such as the NZQA Professions of the respondents that were not readily recognized by NZQA were physician, pediatric nurse, geologist, and accountant. As one participant who was a medical doctor in China stated, “Ah, for me it's very hard to be a doctor, quite hard. So I gave up that idea. I don't want to push [myself]...” (Chinese1F).

She continued to relate this difficulty after several questions:

Oh yeah yeah. You have to get registration. That's the problem. The registration process is very very difficult and takes a long time and lots of money. Yeah, several years and thousands of dollars. And still can't guarantee you get that registration in the end...(Chinese1F)

Moreover, some FGD participants related that in New Zealand qualifications and work experiences earned overseas did not really matter contrary to their initial expectations. For instance, the German group lengthily discussed how it was so "bizarre" not to give importance to qualification like the way it was being considered in Germany. Hence, they were not employed in areas where they can directly apply their skills or educational background. What was important for the employers were the general skills set (i.e. analytical skills, some computer skills, and data-entry skills).

\section{“Kiwi” accent.}

The Kiwi accent is another peculiar specific aspect of the New Zealand culture that immigrants have to learn to adapt albeit in a considerable length of time. Indian participants recalled some foreign nationals being trained to have 
proper Kiwi accents as part of the requirements to gain entry into a job. Zimbabwean respondents who had good command of English had also difficulty finding a suitable job because of this criterion. Thus, a Zimbabwean respondent stated, "I have the better qualification for most people who are above me...in terms of New Zealand qualifications or overseas qualifications? But I definitely was not informed to go over for interview. I had to make my accent slightly better" (Zimbabwean2M). Thus, the participant thought he was highly qualified but was not invited even for a preliminary job interview due to his thick accent.

Proper network.

Another criterion that makes it difficult for immigrants to acquire job easily in New Zealand was having proper networks. Thus, networking or knowing the right set of people in the industry where one intends to be employed was also considered by the participants to be a good gateway or can facilitate entry into the workforce. Having New Zealanders who know you is an advantage in terms of serving as references who could vouch on your communications skills and relationship-building skills. Hence, it is important that an immigrant should learn to mingle with local New Zealanders and get to know the right set of people. This is, however, a hindrance to new incoming immigrants who do not know anybody in organizations and who are depending on the educational background and skills obtained overseas. As one German participant had said:

It's more a question of if people know you. And think you're qualified to do something. That's more important here in NEW ZEALAND than what is...any form of paper, who cares about 
that. As long as they know what you can do, that's it. (German4M).

\section{New Zealand-Specific On-the-Job Barriers to Positive Employment Outcomes}

\section{Mismatch in Education and Qualifications.}

As a stopgap solution, the respondents would accept jobs that either were below or did not match with their qualifications and experiences gained overseas. Some expressed a sense of loss of status because of the experience of having to start from the bottom level or starting another profession all over again. Most of the respondent's current jobs were a result of shifted careers or the current professions were no longer the original professions that they brought in to this country. For instance, there were examples of needing to shift from nursing to university language tutors or lecturers, engineers or marketing people going into IT professionals, policy analysts going to database administration, etc.

The common experience is you keep looking and then you can't find suitable job...[according to] your expectation. You find another... job in another area... you have to work hard yeah...It's very hard to find a job comparable to the job in China. (Chinese1F).

\section{Underemployment.}

Moreover, immigrants should not expect too much if they are still looking for initial employment since in New Zealand, it was a rule of thumb to start from the lowest level regardless of your earned qualifications or work 
experience overseas. As one participant with a Master's Degree who was doing a clerical job in an insurance company stated:

In my company...our educational background, we come from different disciplines... I feel that I don't really belong in this sector because...there are jargons I really didn't know...I just have to learn on the job. And I realized that even if you have this post graduate degree, you just have to prove yourself that you can make it in the job. They don't look at you at face value. They don't look at you, at qualifications really, unless you are in a very highly skilled sector like for example engineers or accountants. (Filipino5F).

Those who were into jobs that were relevant to their professions such as engineers and IT professionals, on the other hand, also felt that they were not yet slotted to the appropriate level or ranking in the organization. Hence, the expressed satisfaction of being into the right profession was somewhat incomplete at this stage.

The struggles are there more than anything else when you are qualified. You're not getting jobs first hand to that level. I'm doing a quite good job now. But still not at the level where I once was. I'd like to be a leader. But I don't do that now. I work for somebody. (Indian4M).

\section{Underpaid.}

There was also an expressed impression that immigrants usually are paid lower than peers who were New Zealanders. They felt that immigrants get lower salary offers and sometimes do not get overtime or holiday pay 
unlike their New Zealander counterparts. This issue was mentioned by some Chinese and German participants. A Chinese respondent shared that she discovered that her pay was lower than that of a New Zealander colleague although they started in the same entry level. She said, "Cause Um I have to work maybe 9 hours a day without any break? And then I just get $\$ 10$ for one hour ... if I'm a Kiwi I can get $\$ 15$ or $\$ 16$ an hour”. (Chinese3F).

\section{Kiwi accent.}

Not having the proper Kiwi accent can still be an issue even if the migrants were already employed. For instance Zimbabwean participants related that "Kiwi accent" could be a barrier to career progression in the organization especially if one's job is a customer-facing job. Hence, they expressed that one needed to learn the proper Kiwi accent in order to be considered for higher positions.

Sometimes you are in the Call Centre, well at least from customers you can take the call. But then, these guys would really look at your accent if they are to give you a promotion. So you will be competing against a Kiwi with a Kiwi accent. (Zimbabwean3M)

\section{Reluctance and distrust.}

Lastly, some participants perceived reluctance and distrust from New Zealanders during interactions. For Chinese participants it was attributed to lack of English language skills. Chinese participants expressed being discriminated by New Zealanders in the form of being abruptly dismissed if the latter did not feel like interacting with them. Hence, the New Zealanders gave the excuse of "not being able to understand" although the Chinese 
respondent was communicating in English. Similarly, Zimbabwean perceived that their employers do not trust them because of their skin colour. They felt as if they were constrained in a box, or being caught in-between two rocks because of these feelings of employers' distrust. For them, there was some sort of a "ceiling" that they cannot surpass no matter how hard they try in their jobs. As one Zimbabwean participant has shared "The assumption is we are not capable. Until you prove yourself. You keep proving yourself." (Zimbabwean1M).

Despite these barriers, some participants commented that once one had crossed the initial barriers of gaining the suitable employment it appears that being immersed in the workplace afforded some acceptance from New Zealander colleagues. The respondents expressed getting acceptance in the workplace and interacting well with their colleagues and superiors. Hence, their cultural background did not seem to matter anymore. New Zealanders were also viewed as accepting and good people. New Zealanders try to accommodate other people's culture such as trying ethnic food recipes and being fond of the taste. Hence, immigrants feel accepted in general social interactions but in the employment context they would still feel a bit rejected. For instance, one Filipina participant related:

We are second class citizens here. And unless you renounce your citizenship and you get a New Zealand passport... Well I don't think it would make a difference but, that's one thing I realize when they see that you are an Asian and you speak differently...you get turned down. (Filipina5F) 
Similar perceptions were expressed by the Zimbabwean group wherein they felt that skin colour continues to be a hindrance to their total integration in New Zealand. As one participant has expressed, "We have to feel we are immigrants. It would be a long time probably. With this kind of treatment, it's not personal. You are always reminded that this is not your place". (Zimbabwean1M).

Thereby, when asked to describe how these experiences affect their settlement, a Zimbabwean summarized his experience as:

I think whole[sic], it invalidate us. Normally we have our own Zimbabweans social gathering...instead of enjoying the party, most people are actually crying about their work situations. So that tends to stress them which in turn [do] not motivate you to participate more in your workplace. So when I asked people looking for another job...looking for another job...looking for another job. Because we are not settled... on our jobs. We are not settled. (Zimbabwean4M).

\section{Summary}

Overall, the participants' responses generated themes that centered on descriptions of culture specific factors and New Zealand -specific factors. Culture specific factors were categorized into positive and negative characteristics inherent to the participants' culture that facilitates or hinder the attainment of positive employment outcomes, which is primarily being hired in a desired job. The Chinese, Filipino and Zimbabwean groups mentioned statements that fell in the negative (barriers) culture-specific characteristics 
while the German and Indian groups mentioned characteristics that were classified as positive (facilitators).

The second factor, New Zealand-specific barriers, was composed of statements relating to practices or policies that were inherent to New Zealand organizations that hinder the immigrants' attainment of the desired job and the perception of being settled in their current workplaces. Some factors would be experienced from pre-employment to post-employment like language, accent and reluctance towards certain ethnic groups. There are also factors that permeate the boundary of pre-employment to post-employment like not having kiwi experience and qualifications not being recognized. These factors lead to mismatch in the migrants' qualifications and work experience gained overseas versus the job that they were handling now.

The factors can be further categorized into formal and informal structures in the two different phases of employment. Formal structures pertain to organizational policies and practices that create the barriers for the immigrant's entry into the workforce or barriers that negate them to attain their highest potentials in their workplace. Informal structures on the other hand pertain to interactions and relationships within the workplace.

Table 4.5 illustrates the summary of the themes generated in the Focus Groups Discussions according to the perceptions on the two structures as they manifest in the two phases of employment (job entry or pre-employment and on-the-job). First, Quadrant 1 (Q1) pertains to institutional recruitment formal requirements or practices hindering the entry of immigrants. Second, Quadrant 2 (Q2) contains the perceptions of the reluctance of majority people to interact with immigrants because of their personal characteristics like skin colour and 
ethnicity during pre-employment phase like job interviews. Third, Quadrant 3 (Q3) contains perceptions of workplace policies and procedures that hinder the attainment of financial and developmental goals of immigrants. Finally, Quadrant 4 (Q4) pertains to perceptions that points out to being unfairly treated or being regarded as dishonest or being regarded with less trust in subordinate-superior relationships and social networks within the workplace.

Table 4.5

Immigrants' Perceptions of Workplace Discrimination in New Zealand

\begin{tabular}{|c|c|c|}
\hline & \multicolumn{2}{|c|}{ Phases of Employment } \\
\hline & Job-Entry & On-the-Job \\
\hline & (Q1) & (Q3) \\
\hline \multirow{4}{*}{ Formal Structures } & Language/Accent & Language Accent \\
\hline & Overseas qualifications not recognized & Mismatch (Job vs. Qualifications) \\
\hline & "Kiwi" experience & Inequity in Pay \\
\hline & (Q2) & (Q4) \\
\hline \multirow[t]{2}{*}{ Informal Structures } & Reluctance towards colored people & Distrust for colored people \\
\hline & Network/Knowing people & \\
\hline
\end{tabular}

Note:, $Q=$ Quadrant

\section{Discussion}

This study was conducted to gather immigrants' workplace perceptions in the New Zealand context and explore the prevalence of PD in New Zealand organizations coming from immigrants' perspectives. Further, concepts that could later on be included in the construction of a scale were explored through the FGD. It was aimed to answer the following specific questions (1) how do immigrants perceive the New Zealand workplace, (2) what experiences are 
specific to certain groups, (3) what experiences are common to immigrants; (4) what can be concluded based on these perceptions?

The findings show that the participating immigrants view New Zealand workplaces as egalitarian and relaxed. This overall view further facilitates relational dynamics towards being less formal (e.g. without the use of titles referring to the calling the boss) and having a more relaxed working relationship with co-workers and peers (e.g. New Zealanders are generally viewed as open and accepting about other people's cultures like trying ethnic food). In this sense, migrants regard New Zealand workplaces in a positive light.

Experiences specific to immigrants groups relate to employment concerns like lack of competency in the English language (Chinese), not being assertive in interviews (Filipino), facing racism which leads job entry and promotional barriers (Zimbabweans), straightforwardness and commitment to the task (Germans) and being pragmatic in choosing careers (Indians). Moreover, the negative perceptions leading to some discriminatory practices were more focused on institutional policies and practices. This is consistent with previous research that individuals perceived more institutional discrimination than personal discrimination (Ensher et al., 2001; Watts \& Carter, 1991). Particular experiences of the immigrants related to institutional or organizational level discrimination are non-recognition of education and work experience, requiring immigrants to have local New Zealand experience before being considered for a job, claims of underemployment and underpayment, and irrational employment requirements like having the right accent or knowing people in organizations. These are the common experiences 
of the immigrants in the workplace that were regarded as discriminatory practices as defined by the participants themselves.

Furthermore, there were two conclusions that can be drawn from the analysis of the themes generated from the transcripts of the focus groups discussion. First, the respondents' perceptions are subtle forms of employment discrimination that could happen from the job-hunting phase and up to the current employment. At the onset, PD may have come about because they are new to the host country thus, they may have experienced unfair treatment in looking of jobs or working for the dominant majority group because they are dissimilar in terms of the physical characteristics, language or cultural background. The Similarity-Attraction-Attrition (ASA) model of Schneider (1987) explains that organizations will tend to hire people who have the same characteristics and those who feel different will eventually leave leading to homogeneity of the remaining members. The homogeneity of the remaining members of the in-group will also have an effect on their conceptual and behavioural reactions to the members of the out-groups thus they will be attracted only to similar others (in-group). This becomes a vicious circle leading to the exclusion of the members of the out-groups. Thus, Riordan et al. (2005) argue that ASA can explain the formation of in-groups and out-groups within an organization like social groups, informal relationships and mentorship.

Specific forms of PD were observed in the studies that were consistent with the current New Zealand literature (see Basnayake, 1999; Podsiadlowski, 2006; Selvarajah, 2004; Ward \& Masgoret, 2007). These practices of institutionalized discrimination were in the form of not being able to or 
difficulty in obtaining one's intended career objectives because of "inexplicable" barriers or requirements from the New Zealand institutions and organizations. This may be in the form of low competencies in the English language or not having appropriate communication skills, not having the proper accent, not having New Zealand -recognized qualifications, no New Zealand -based work experience, distrust for dark-coloured individuals and the like. Therefore, meeting the New Zealand -specific entry requirements, such as New Zealand -recognized skills and experience (mostly those that will equip an employee in performing unstructured jobs and interacting mostly in team-based work environment) and the New Zealand work experience (Kiwi experience), are crucial in getting meaningful employment. These requirements are quite difficult to attain for the recent arrivals. Thus, most new entry immigrants have to be content with menial jobs to earn a descent living. It is also a rule of thumb to start from the lowest level regardless of your earned qualifications and previous work experience. The majority of participants had to start from entry level for the jobs they wanted to get into. New Asian and Migrant Health Research Centre (2004) would describe this as a common experience for migrants in the first two years of their stay in New Zealand.

The second conclusion of this study is that the themes formed from the respondents' experiences in the New Zealand workplace were parallel with previous categorizations in the organizational literature with additional variation. Specifically, themes in this study were mainly categorized into experiences during job-entry phase and on-the-job phase. These two phases were consistent with the two components of discrimination in the workplace 
identified by Levitin and colleagues (1971). Access discrimination was described as discriminatory experiences during hiring or pre-employment stages such as those related to denial of job offer and low starting salary while Treatment discrimination is considered as discriminatory experiences upon employment like promotions and salary increases. The Job-Entry themes reflect aspects of Access Discrimination while the On-the-Job corresponds to the descriptions of Treatment Discrimination.

FGD themes were also consistent with the Formal and Informal employment discrimination earlier categorized by Levine and Leonard (1984). To reiterate, Formal discrimination refers to the organizational policies related to hiring, compensation, and benefits while Informal discrimination pertains to relational dynamics or interactions and work atmosphere.

Both Access and Treatment Discrimination were categorized by Chung (2001) under Levine and Leonard's (1984) Formal employment discrimination. In my study finding on the other hand, some aspects of access discrimination (job-entry) and treatment discrimination (on-the-job) can be categorized in both formal and informal discrimination. This variation is deemed an important contribution to the organizational psychology literature as a new model for understanding the perceptions of discrimination of immigrants in the workplace.

\section{Limitations of the Study}

The first limitation of the study is the generalizability of the findings due to the small number of participants compared in proportion to the total ethnic immigrant population of New Zealand. Thus, we cannot claim that we 
had a representative sample of the total migrant population. Representatives from other ethnic groups may have different views regarding the same issues that were not captured in the FGD.

Secondly, we did not have a way to measure reliability of the data since aside from the very small sample sizes, the responses are in nominal form (e.g. verbalized comments and other non-verbal behaviors such as facial expression and gestures) hence preventing the analysis in statistical format. Third, there is always a question of subjectivity in the interpretations of the data. These three limitations are inherent to FGD as a qualitative method thereby giving some precaution the interpretation and generalization of the data (Hair et al., 2006).

Fifth, the exploration of other demographic and organizational demographics may provide additional explanation of immigrants' perceptions. Specifically, the current sample may have started working in lower level jobs but they were already in professional and administrative positions as of the time of data gathering. Experiences of migrants who came to New Zealand under the skilled immigrant category who are in the lowest level of the job hierarchy are also a better venue of investigation regarding perceived discrimination in the workplace.

Sixth, although the originally intended method of participant recruitment was random selection, the resulting selection procedure turned out to be snow balling technique and convenience sampling because of the difficulty of getting employed people to sit in the FGD. Among the difficulties observed were time constraints because most of the intended participants worked during the day and their free time after office hours was usually 
devoted to the family. Furthermore, this activity was done without compensation hence those who report for work during evenings cannot be tapped. Hair et al. (2006) consider randomization as a measure that can assist to minimize selection bias but they also opine that there is no real guarantee for this. Thus they point out that randomization is not as critical in qualitative research methods such as FGDs as in quantitative surveys because what is essential is participant's credibility and having met the established selection criteria. Overall, all these encountered difficulties are not new since other researchers have also expressed that it is a challenge to get accurate samples from ethnic minority populations (e.g. Cassidy et al., 2005; Hughes, Fenton, Hine, Pilgrim, \& Tibbs, 1995).

\section{Suggestions for Future Research}

A follow-up quantitative study that could validate the findings of this study would address most of the limitations identified above. First, using a large sample could improve the generalizability, reliability and validity of PD as understood and experienced by target groups. Further, additional FGDs can also be conducted to get the views of other ethnic groups that were not represented in this study. Some ethnic groups may have more positive or more negative experiences that could enrich the data of this study. The results show that the participants perceived subtle forms of discrimination in the job-entry and on-the-job stages of employment although the participants generally perceive the social environment as accepting and egalitarian. This contradiction is worth exploring further in future studies either through 
qualitative methods of investigation such as interviews and FGDs or through surveys of bigger samples to get a more representative population.

\section{Summary and Conclusions}

This chapter presented a study of the workplace perceptions of immigrants in New Zealand. The qualitative exploratory study investigated the prevalence of discrimination based on immigrants' workplace experiences. Thus, the significant contribution of this study is the systematic analysis of immigrant participants' experiences and perceptions of unfair treatment in the workplace. The findings were consistent with the existing conceptual literature on general PD in the workplace. A new model for understanding the stages and forms of PD reported by immigrants in this sample is deemed an important contribution to the literature since it integrated the basic forms and phases of discrimination mentioned in the literature.

Furthermore, it has been suggested that PD, in contrast to actual discrimination, has an important impact on a person's assessment of what would be preferable as the coping strategy (Chung, 2001). Some participants were referring to their experiences as reflections of subtle forms of discriminatory practices while others specifically point them out as racism. Nonetheless, the overall take home message for this study is that the participants perceived some discriminatory practices in New Zealand albeit they generally view the social environment as accepting and equality-driven. However, this acceptance in terms of being interested in the immigrants' culture such as tasting ethnic food is considered only as symbolic but not realistic acceptance. The egalitarian climate in the workplace seems superficial 
since it only pertains to name-calling (e.g. no titles such as "sir" or "boss') and does not really translate in terms of promotional opportunities for the immigrant groups. These contradictions are worth investigating in more indepth and wide-ranging studies that will be undertaken by the next chapters.

Thereafter, the PD concepts generated in this study will be integrated in the construction of a PD scale relevant for immigrants and eventually for employees in general. This scale is aimed to capture the experiences perceived as discriminatory by target groups in their workplaces. The next three chapters will tackle the creation of the PD scales and testing its concurrent and predictive validity with other covariates. 


\title{
Chapter 5
}

\section{SCALE CONSTRUCTION AND VALIDATION (STUDY 3-A)}

\author{
Introduction
}

The previous chapter (Chapter 4) has generated several concepts/themes on the nature of discrimination in the workplace as perceived by immigrants. It would be interesting to test if these themes can be measured in survey format. This chapter outlines the steps taken to search for an appropriate perceived discrimination (PD) measurement/scale that captures the themes generated in the focus groups discussions. The first half of the chapter reviews the existing measurements on race/ethnicity related discrimination issues. Since there was no existing single scale that matched the themes from the focus groups, the second half of this chapter outlines the steps taken to construct new PD in the workplace measurements/scales that are used in the succeeding chapters to generate more generalizable and statistically reliable data on PD and its correlates. Overall, the current chapter presents the quantitative investigation of the nature of $\mathrm{PD}$ from the immigrants' perspectives.

As mentioned in Chapter 3, the overall research strategy of this thesis is to combine qualitative and quantitative research methods since it is acknowledged that each research method has its own advantages and disadvantages. An in-depth, qualitative investigation into the nature of PD was examined through focus groups discussions to provide a deeper understanding of discrimination based on the direct experiences of immigrants in the workplace. Quantitative measures, on the other hand, are useful in capturing a broader population in structured questionnaires to further investigate 
underlying constructs as well as possible relationships of these constructs to other variables. Thus, through the presentation of quantitative surveys in the next three chapters, it is aimed that perceptions of a larger sample of participants is captured to generate more generalizable and statistically reliable data on perceptions of discrimination.

\section{Review of Related Literature on PD Measurements}

Simultaneous with the preparation and conduct of the focus groups discussion, the literature on discrimination was reviewed and evaluated for measurements used in studies related to PD as a function of race and ethnicity. The main objective of this review is to identify existing scales that will be appropriate for measuring PD of targets' perceptions (employees in general and immigrants in particular) in the workplace setting. The focus was on constructs that relate to the cognitive aspects of an individual's personal experience or perceptions about discrimination. A particular emphasis was placed on measures related to the following: workplace or job-related issues, ethnicity, migrant status, minority status, personal and organizational demographics, and organizational structures.

\section{Existing Measurements on Race or Ethnicity-Related Discrimination}

Scholars interested in this area have used a number of instruments to measure aspects of race or ethnicity-related discrimination from the target's perspective in different domains such as social, public, academic, and the workplace. Upon closer look at the discrimination literature related to ethnic and race issues, there were four main approaches to measuring or assessing perceptions or experiences of discrimination. These were (a) personal and 
group perceptions, (b) discrimination due to group membership, (c) lateral, vertical and systemic discrimination, and (d) frequency of discrimination. The first two approaches can be considered as individual-level methods of measuring discriminatory perceptions or experiences. These methods measure the individual's perceptions, either on a personal level (self) or as member of a race or ethnic group (dual self). Moreover, the third approach is a relational method of determining discriminatory cognitions. This approach looks into the perceptions originating from interactions with other people belonging to different social categories such as peers (lateral), superiors (vertical) and the societal/organizational structures (systemic). The last category relies more on the rate of recurrence or frequency of the discriminatory experience. An illustrative presentation of these four approaches is presented in Figure 5.1.

\section{Figure 5.1}

Existing Measurements Approaches for Ethnicity/Race Related Discrimination

\begin{tabular}{|l|l|}
\hline $\begin{array}{l}\text { 1. Self: Race Or Ethnic Group } \\
\text { Identification }\end{array}$ & $\longrightarrow$ \\
$\begin{array}{l}\text { 2. Dual Self: Personal Vs. Group } \\
\text { Perceptions }\end{array}$
\end{tabular}

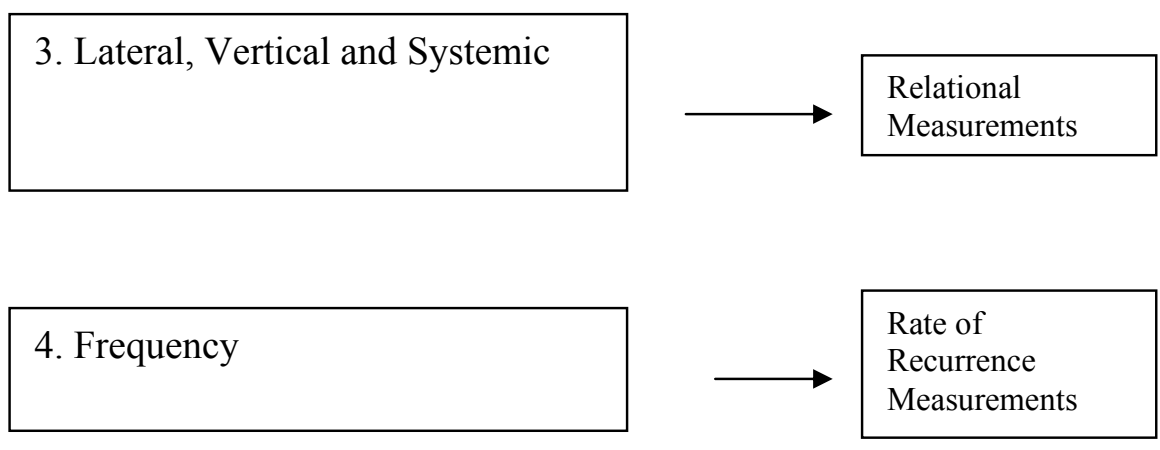




\section{Personal vs. Group Perceptions}

There are ongoing discussions by scholars regarding the need to give appropriate attention to measuring discrimination as individual-level perceptions versus group-level perceptions. Currently, more studies show that individuals tend to perceive a higher level of discrimination directed to their group rather than on a personal level (Barry \& Grilo; 2003, Dion \& Kawakami, 1996; Taylor et al., 1990; Taylor, Wright \& Ruggiero, 2001). There have been several explanations offered regarding the discrepancy between perceptions of personal and group discrimination.

First, the personal/group discrepancy was explained through three categories by Taylor et al. (1990) and further developed by Taylor et al. (2001). Two of these categories are motivation-based and the last one is cognitive-based. The initial category looks into the motivations of denying or minimizing personal discrimination. It refers to Crosby's (1984) rationalization that reporting of personal discrimination is usually difficult due to the complexity of ascertaining reasons for discriminatory experiences on an individual basis (whether it is due to self or organizational factors), and because people usually find it difficult to confront their own victimization. The second category is the individual's intent or motivation to exaggerate group discrimination. They posit that members of target groups may exaggerate the group's discrimination experiences in order to promote social change and improve the conditions of the group. The third category is attributed to the cognitions of the individual and the manner that the information is processed cognitively. Group discrimination perceptions are suggested to be higher since the individual may be adding his or her own 
experiences to those of other group members. Related to this is the concept of "proportionality", which is described as the process in which individuals are gauging their own experiences in proportion to their group's experiences. Hence, if individuals are assessing their group's experiences as not that significant or in minimal terms, their own assessment of their personal experiences may appear as even less significant.

The underlying notion of the above-mentioned categories is that group PD is higher because of the individual's outright denial or minimization of a negative personal experience. In contrast, the second major explanation offered by the Discounting Hypothesis/Model by Crocker and Major (1989) provides a more positive outlook to the personal and group discrepancy by referring to the individual's coping mechanism as the reason for higher group PD. The Discounting Hypothesis suggests that engaging in external attributions by individuals instead of internal ones are self-protective strategies to shield the self-concept in the event of personal failure. According to Crocker and Major's (1989) review, personal attributions by individuals being discriminated leads to negative effects on well-being. Thus, they posit that it is more acceptable for the individual's self concept to blame a racist employer as the reason for the failure to get the job than to look into his own lack of personal qualifications.

Most of the scales relating to personal and group perceptions discrepancy that were reviewed in this study were constructed by the authors themselves. For example, Levin, Sinclair, Veniegas, and Taylor (2002) created their own single-item measures for personal and group discrimination under the general, gender and ethnic discrimination categories. Romero and Roberts 
(1998) used a 2-item scale measuring the amount of PD against own ethnic group and personal experience. However, in their analysis they did not breakdown the group and personal components of the scale. They just reported the responses on perceptions of discrimination as a single scale. Likewise, a 2item scale constructed to assess discriminatory perceptions or experiences on a personal or group level was developed by Barry and Grilo (2003) for their study on East Asian immigrants in the United States.

Another example is the 7-item scale constructed by Bourguignon and colleagues (2006) used to measure PD of Africans in Belgium. Furthermore, Spencer-Rogers and Collins (2006) measured group discriminatory perceptions of Latino undergraduate and graduate student in the US using a scale they constructed in 2001 - the Racial-Ethnic Group Disadvantage Scale (REGDS). This scale measures PD on a group level related to the ethnic group's relative status in the social, economic and political milieu.

Other authors opted to adapt items from existing validated scales. Rollins and Valdez (2006) used the Racism and Life Experiences Scale (RaLES) to look into personal and group discrimination of African American high school student samples. For various domains, two scales were developed by Harrel (1997a; 1997b, in Rollins \& Valdez, 2006). The Racism and Life Experiences Scale-Personal (RaLES-PER) and the Racism and Life Experiences Scale-Group (RaLES-GRP) measure personal and group aspects of racism. RaLES-PER is a 20 -item scale covering personal perceived racism beliefs in different areas of life like job search, self-esteem and emotional well-being and the RaLES-GRP is a 16-item scale looking into perceptions of racism on a group level. Similarly, Shorey et al. (2002) adapted two items 
from the Kobrynowicz and Branscombe's (1997) gender discrimination study to measure personal discrimination and another two items from the group perceptions of gender discrimination scale. Shorey et al. (2002) replaced "gender" with "race" in the items and used these two 2-item scales to measure perceived personal and group discrimination related to race of 126 Hispanics and 153 Anglos from a US university.

Studies using the measurements mentioned above have found lower perceptions of personal discrimination than that of group discrimination among East Asians in the United States (Barry \& Grilo, 2003), African immigrants in Belgium (Bourguignon et al., 2006), African American high school students (Rollins \& Valdez, 2006), female Canadian university students (Taylor et al., 2001) and Indian and Haitian women in Canada (Taylor et al., 1990). Similar study findings from Shorey et al. (2002) showed that the Hispanic participants had higher perceptions of group discrimination than perceived personal discrimination. Their Anglo participants, however, did not show any difference between their personal and group perceptions. Levin et al. (2002) study outcomes, on the other hand, illustrated that there were personal/group discrepancies for White women and White men but not for female and male Black Africans and Latinos.

Overall, the above findings show that generally individuals tend to report lower levels of perceived discrimination on a personal level compared to perceptions attributed to their referent group or to the organizations to which they belong. Thus it may be true that individuals have a view of personal invulnerability although they still experience distress in the event of painful experiences (Janoff-Bulman, 1992). 


\section{Group Identification: Race or Ethnicity}

Scholars are gradually more in agreement that race is not determined by biological distinction but "a gross indicator of distinctive social and individual histories" (Williams, Spencer, Jackson \& Anderson, 1999, p.71). Therefore, race and ethnicity are considered social categorizations (Williams, 1997). Studies on race-related or ethnicity-related discrimination usually refer to social psychological theories of intergroup relations such as Social Identity Theory (Tajfel \& Turner, 1979; 1986) and social categorization theories (Turner, 1985) for explanations on people's propensity to segregate themselves into demographic groups (e.g., race, ethnicity).

Discrimination due to group membership is akin to personal PD which results from unfair actions affecting the person at the individual level. Generally, studies measure personal discrimination as a direct function of race/ethnicity (McWhirter, 1997; McWhirter, Hackett, \& Bandalos, 1998; Spencer-Rodgers \& Collins, 2001). Most scales constructed in this category were intended to measure PD of specific groups such as Blacks and Hispanics. These scales or selected items were eventually modified to fit other race/ethnic groups such as Asians. Moreover, all items in these scales are intended to measure solely personal PD unlike the scales mentioned in the personal and group perceptions category, wherein a combination of personal and group items are simultaneously found in the scale.

Several scales measuring discrimination perceptions and experiences linked to one's race or ethnic background have been developed and used in studies. One of the most studied races in the United States is African Americans and several scales have been developed initially for looking into 
their cognitions. One example is the Perceived Occupational Barrier Scale ([POBS]; McWhirter, 1997) which is a 24-item Likert type scale designed to measure anticipated ethnic and gender-related barriers in education and career. It has been used for example by Constantine, Wallace, and Kindaichi (2005), Lease (2006) and Flores, Navarro, Smith and Ploszaj (2006). Another scale is Turner and Turner's (1975) Perception of Discrimination Against Blacks Scale which contains items that assess Black peoples' access to 21 professional occupations (e.g. accountant, law, teacher). This scale was used by Evans and Herr (1994) in their study on African Americans student sample to measure their levels of perceived discrimination.

Another measure that looked into references of discrimination due to race or ethnic background was developed by Fried et al. (2001) to assess experiences of racial discrimination of African American university students. Jefferson and Caldwell (2002) designed the Discriminatory Scenarios Questionnaire (DSQ) for African Americans and tested it on student samples. The DSQ is a 10-item questionnaire depicting hypothetical scenarios. It measures the level of perceived bias on blatant discriminatory acts committed by hypothetical White and Black Americans on individuals from another ethnic group. The pattern of the scenarios was based on items previously created by Rodin et al. (1990). One scale developed to assess indirect reference to discrimination is the Modern Racism Scale (MRS) by McConahay (1986). Slaughter et al. (2005) adapted 4 items from the MRS to measure perceptions about how would White counterparts treat Blacks in the workplace. Slaughter et al.'s (2005) 4-item scale was used to measure perceived racism among a large sample of Black American Engineers 
$(\mathrm{N}=2,480)$. In a more specific context like the workplace setting, a 5-item workplace-based scale, adapted from Workplace Prejudice/Discrimination Inventory ([WPDI], James et al., 1994) was used by Slaughter et al. (2005) to measure experience of discrimination by Black American Engineers.

Aside from African Americans, PD measures attributable to one's ethnic background have also been developed for other groups such as Hispanics. Sanchez and Brock (1996) developed a 10-item Perceived Discrimination Against Hispanic Scale relating to workplace perceived unfair practices targeted at individuals of Hispanic-origin. They selected these items from Mena, Padilla and Maldonado's (1987) Acculturative Stressors Scale and added "at work" at the end of the items to suit the context of the study. Subsequently, Foley et al. (2002) adapted Sanchez and Brock's (1996) scale for their study on Hispanics in the legal profession. In another study, Foley and Kidder (2002) modified the Sanchez and Brock's (1996) PD scale into a 5-item scale to measure perceptions of ethnic discrimination of Hispanic Law students in the US. For a much younger Latino sample, Romero and Roberts (2003a) developed the Bicultural Stressor Scale (BSS) which they used to measure everyday life stressors of 881 Mexican-descent middle school students and the effects on self-esteem. Using the same sample, Romero and Roberts (2003b) used a subscale of BSS to measure perceived discrimination and its effects on self-esteem and ethnic identity.

Scales were also constructed for ethnicities other than Black Africans and Hispanics to suit other groups like Europeans, Asians and more diverse groups. Having adolescent samples in the Netherlands, Verkuyten and Brug (2002) assessed the extent of being confronted with discrimination using a 
scale which they adapted from the items of Ruggiero and Taylor (1995) in their study of Dutch and Surinamese students.

A 3-item PD measure was developed by Finch et al. (2000) and subsequently used by Lee (2003) for his study on Asian American students of Indian descent. These items were originally obtained by Finch et al. (2000) from the Hispanic Stress Inventory (Cervantes, Padilla \& Salgado de Synder, 1991). Lee (2003) modified the items by replacing the ethnic reference group from Mexican to Indian. Another scale used for more diverse groups was developed by Foster and Matheson (1995). The scale is a 7-point Likert scale containing items which measure personal experiences of discrimination due to their ethnic or group membership. Foster et al. (2006) used this scale to measure the levels of personal discrimination experienced by university students coming from various ethnicities like African Americans, EuropeanAmericans, Asian Americans, Latin Americans and others. Lastly, Konrad and Spitz (2003) developed a 3-item measure for perceived race discrimination which they adapted for a study involving White and "men of colour" participants. Men-of-colour was a collective name they assigned to those who identified themselves as African Americans, Hispanic Americans, Asian Americans and Native Americans.

Overall, the above-mentioned scales were used in studies to gauge the extent of discriminatory perceptions of specific groups in different settings like the workplace, school or society in general using different populations like students and professionals. Moreover, these scales were also used to compare the levels of PD between specific groups and a referent group (usually White participants). 


\section{Lateral, Vertical and Systemic Perceptions of Discrimination}

Measurements developed to assess the different hierarchies in which discrimination may occur can be categorized into (a) lateral, (b) vertical, and (c) systemic. Lateral perceptions pertain to the individuals' perceptions of discrimination as a result of interacting with people within their own rank like colleagues/co-workers or peers. Vertical perceptions refer to perceptions coming from interactions with people who are above in status like supervisors or teachers (for students). Lastly, systemic perceptions come from institutionembedded discriminatory acts like organizational policies and procedures. Reskin (2000) argued that organizational practices are the proximate source of work discrimination motivated by mostly unconscious cognitive bias towards in-group favouritism.

Multi-level analysis of discrimination perceptions specifically in organizational research stemmed from the integration of demographic variables and contextual factors (i.e. organization) with the belief that group identification does not occur in isolation (Ensher et al., 2001). Hence, group identification based on demography as supported by Social Identity Theory (Tajfel \& Turner, 1979; 1986) and Relational Demography Theory (Tsui et al., 1992) was complemented by identification with organizational groups (people with similar tasks, function or hierarchical status). The Intergroup Theory (Alderfer et al., 1980) thereby combines group membership and environmental context to explain how individuals try to balance competing expectations from their identity group and the organizational groups. In order to further understand the effects of the PD process, the intergroup theory was expanded to Embedded Intergroup Theory ([EIT], Thomas \& Alderfer, 1989), which 
points to the intricate nature of relationships within organizations such as interactions with supervisors, co-workers and organization as a whole. These three levels of intergroup relationship network are mirrored by the Interactional Model of Cultural Diversity (IMCD) by Cox (1993). This model was initially used for measuring effects of diversity. The IMCD similarly proposes three levels of analysis in terms of group affiliation (e.g. race, ethnicity), namely, (a) individual (b) group-intergroup and (c) organizational. IMCD is also useful framework in studying multiple levels of PD (Ensher et al., 2001)

The significance of measuring PD at different levels has been pointed out in the literature since different levels have differing effect on various outcomes (Ensher et al., 2001). To show that these different levels of perceptions exist, past research has confirmed that discrimination can be experienced by individuals at different levels. Previous studies measured perceptions of unfair treatment from interactions with supervisors/adults, peers/co-workers and from an organizational level perspective in terms of policies and procedures (e.g. Ensher et al., 2001; Waters, 1994; Watts \& Carter, 1991), and societal or labour market structures (Ensher et al., 2001, Jasinskaja-Lahti \& Liebkind, 2001; Way, 1997).

Specifically, assessment of the three levels was undertaken in research by Ensher et al. (2001). They developed a PD scale with three distinct factors, namely perceived co-worker discrimination (4 items), perceived supervisor discrimination (4 items) and perceived organizational discrimination (3 items). The scale exhibits the three categories described above as lateral, vertical and systemic respectively. The researchers used this scale to measure PD with 
employees from various ethnic groups, such as Latino, African American, Asian, and European descent. Outside the workplace setting, levels of PD have been measured in terms of interactions with adults and peers. Way (1997) developed two scales, with 7 items each, to assess perceived ethnic and racial discrimination from adults (vertical) and peers (lateral). The scale was developed from interviews with more than 150 Black, Latino and Asian American adolescents. The scale was used by Greene et al. (2006) in a longitudinal study involving students with various ethnic backgrounds, including Blacks, West Indians, Chinese Americans, and Puerto Ricans.

Konrad and Spitz (2003) measured PD at the systemic level in a diverse group of faculty members from colleges and universities using a 3item scale they named the Belief in Labour Market Discrimination. This scale was adapted from Tougas and Veilleux's (1990) items, which gauged respondents' beliefs about the existence of racial discrimination. The items measure belief in racial discrimination in the areas of hiring and salary, promotion and tenure decisions, and student evaluations, which would fall into the "systemic" category. Personal and institutional perceptions of discrimination of Black civil service employees were also measured by Watts and Carter (1991) using the institutional-racism items adapted from Barbarin and Gilbert (1981, in Watts \& Carter, 1991) and the personal discrimination scale developed by the authors.

Thus, personal PD could come from interactions with co-workers and superiors, and general perceptions of unfair treatment in organizations or in the society (Barry \& Grilo, 2003; Bourguignon et al., 2006; Harrell, 1994, 1997a, 1997b, in Rollins \& Valdez, 2006; Kobrnovicz \& Branscomb, 1997; 
Romero \& Roberts, 2003; Watts \& Carter, 1991). Systemic discrimination, like institutional racism, is sometimes termed as "everyday" racism (Essed, 1991) because of its consistent and persistent unfair effects on target groups (Cox, 1993; Ensher et al., 2001). These generalized perceptions are structurally embedded unfair practices from discriminatory policies and procedures that are subtle and sometimes not intentional (Essed, 1991).

In general, perceptions directed at organizations or systemic PD generate higher levels of responses. Specifically, the comparative effects of these levels were shown in the study of Watts and Carter (1991) wherein they measured racial discrimination and personal discrimination (related to job selection, promotion, and performance evaluation) involving AfricanAmerican government employees. They found that institutional racism was given more importance by respondents than discriminatory perceptions on personal level experiences. Similarly, Ensher et al. (2001) measured PD discrimination at three levels namely co-worker, supervisor and organisation from an ethnically diverse sample of employees in the US. They found that organizational level PD is associated with more outcomes like job satisfaction and organizational commitment.

\section{Frequency of the Discriminatory Perceptions or Experience}

Drawing from the conceptual framework on stressors suggested by Lazarus and Folkman (1984), prejudice and discrimination have been classified as stressors for minority groups (Cassidy et al., 2005). The amount of exposure to these stressors has been found to have differing consequences for individual outcomes such as health (Taylor \& Turner, 2002) and selfesteem (Verkuyten, 1998). There is growing evidence that stress due to 
repeated exposure to discrimination (Clark et al., 1999) is one of the underlying reasons why PD is linked to behavioural problems and depression (Brody, 2006).

The study on the effects of frequent exposure to discrimination (as a stressor) has been linked to various psychological variables that could affect individuals' vulnerability to instances of unfair treatment. There are personality-related factors and other variables that are linked to individuals' higher propensity to see events as discriminatory compared to others. For instance, Phinney et al. (1998) found an indirect negative relationship between self-esteem and PD, such that self-esteem negatively predicted depression which in turn positively predicted PD. Moreover, Kobrynowicz and Branscombe (1997) attributed PD to social approval. Crocker and Major (1989), on the other hand, referred to group membership centrality as a factor that could affect attributions to instances of discrimination. There are further constructs linked to PD that will not be covered by this review due to lack of relevance (e.g. locus of control). Nonetheless, looking into the variables that assess how individuals would appraise a situation, whether it is a one-time experience or a major event in one's life, prompted scholars to construct PD scales that measure time-bounded experiences/events of discrimination.

Some existing measures have been commonly used for African American samples in the literature of discrimination. One of these is the Schedule of Racist Events ([SRE]; Landrine \& Klonoff, 1996), a 13-item scale initially designed to measure Black adult experience of racism for the past year in various context such as the workplace setting. SRE was revised by Brody et al. (2006) into a 13-item scale measuring African American youth's 
perceptions of specific discriminatory behaviours in the community. Gibbon et al. (2004) just used 3 items from the SRE to measure perceived racial discrimination of their African-American parent-child sample.

Another scale is a composite of the Racism and Life Experiences Scale (RaLES) by Harrel (1997; in Rollins \& Valdez, 2006; in Scott, 2003) which was developed to measure the frequency and impact of race-related unfair practices in daily life. One subscale was named the Daily Life Experiences (DLE-R), and this 20-item 5-point Likert scale type was used by Rollins and Valdez (2006). Scott (2003) reduced the DLE-R scale to a 10-item scale for his study on African American students in order to lessen the time to complete the questionnaire. This reduced scale was again used by Scott and House (2005) in another study with Black high school students in the US. This time the DLE-R was termed as DLE-F although the complete name of the scale was maintained as the Daily Life Experiences Scale. Another version of the DLE (18-item version) was used by Sellers and Shelton (2003) to measure the frequency of experienced discrimination in a longitudinal study conducted with 349 first year African Americans college students. In a later study, another DLE version (17-item) was used by Sellers et al. (2006) to measure the frequency of experienced racial discrimination of 314 Black American high school students.

The third commonly used scale is the Perceived Racism Scale (PRS) originally designed for African American samples by McNeilly, Anderson, Armstead, Clark, Corbet, Robinson et al. (1996). It is a 51-item Likert type scale that measures the frequency of perceived racism in different settings like the workplace, school, public domain, and the exposure to racist statements 
over the past year and lifetime. It measures three dimensions of racism for the four above-mentioned settings, namely (a) assessing the frequency of exposure to racist events or statements, (b) emotional responses to racist incidents and (c) coping responses to racist experiences. Combs, Penn, Cassissi, Michael, Wood, Wanner et al. (2006) used PRS for predicting paranoia among African American student sample. Eventually, the scale was used for participants other than Black African-descent like the study of Hall and Carter (2006). This study used the first section of the PRS scale for determining the relationship of perceptions of racial discrimination and ethnic/racial identity of AfroCarribean (Jamaican American) participants. The PRS was also replicated in the construction of scales like the Perceived Racism Scale for Latina/os ([PRSL]; Collado-Proctor, 1999). The PRSL consists of 34-items measuring the frequency of exposure to discrimination of Latino in the domains of employment, academic and public domains. Moradi and Risco (2006) subsequently used the PRSL scale for their study with 128 Latina/os with ages ranging from 18-71 years.

Although not a widely used scale, Taylor and Turner (2002) measured the day-to-day experience (minor daily discriminatory events that do not have lifetime impact) and major events (events that affect the individual in a significant way that could extend to a lifetime experience like being discriminated in career progression leading to lower well-being) using items constructed by Williams et al. (1997). Their sample centred on African Americans and white non-Hispanic sample with ages 25 and over.

Instead of using existing validated scales, some researchers developed their own measures for assessing frequency of perceived or experienced 
discrimination. Deitch et al (2003) developed a 10-item measure for their study on Black and White Americans, which they called Everyday Mistreatment Scale (EMS). This scale focused on the frequency of the occurrence of the different types of mistreatment. Items in the EMS included gauging the incidence of mistreatment and its frequency but did not directly make attributions to discrimination or prejudice. Phinney et al. (1998) also constructed their own scale which measured the frequency of PD through a 7item scale that was developed for their study on Armenians, Vietnamese and Mexican American high school students. The items on this scale cover perceived unfair treatment by peers, teachers and adults and feelings of rejection from the society due to one's ethnicity.

Martin, Tuch and Roman (2003) likewise developed their own scale measuring lifetime (5 items) and current discrimination (4) items in the school and workplace setting for African American samples. The responses to the scale are coded as yes or no and then summed to produce a composite measure of discrimination. They used this scale to correlate PD with problematic drinking among Blacks. They found that the most commonly reported discrimination was in schooling, hiring, promotions and unfair treatment on current jobs. Report of direct discrimination was also found to have a direct effect on problematic drinking.

Similarly, Cassidy et al. (2005) created a PD scale based on frequency of being treated unfairly and feelings of being rejected by the society due to ethnicity which he based from the measures of Verkuyten (1998) and Phinney et al. (1998). They used this scale to measure PD of 154 Chinese, Indians and Pakistani youth (14-21 years old) in Scotland. Jasinskaja et al. (2007) did a 
study on Russian-speaking and Estonian-speaking adult immigrants in Netherlands and used a 4-item scale measuring frequency of perceived ethnic discrimination at work covering the areas of job search, career advancement, dismissal from the job and racial offence/harassment at work. The range of responses was from 1 (never) to 4 (five times or more). Lastly, Zlobina et al. (2006) also measured frequency of negative treatment against 518 first generation immigrants in Spain using a self-constructed 6-item perceived discrimination scale. Moreover, Jasinskaja-Lahti and Liebkind (2001) also developed a 9-item scale for their Russian-speaking adolescent immigrant sample in the Netherlands. The scale consists of items measuring experiences and frequency of being teased, threatened, and being rejected due to one's ethnicity by teachers and peers within their own school, and by other adults and youth outside school. Overall, the instrumentality of frequency scales was demonstrated in studies that linked PD to psychological health outcomes in different context.

\section{Measurement Issues}

After a thorough review of existing literature to search for PD scales that are appropriate for the current focus of the study (i.e. immigrant experiences in the workplace), there was no single scale that was found satisfactory. What the current research intend to adapt is a scale or scales that can capture perceptions of discrimination in the workplace of multi-ethnic groups as characterized by immigrant groups. Moreover, it was desirable to use a PD scale that captures the types and forms of discrimination identified in the FGD like access and treatment discrimination as well as the informal and formal forms of discrimination. However, after a thorough evaluation of the 
existing PD measures related to race and ethnicity, no single scale was deemed appropriate for measuring PD of immigrants. Below are the issues identified during the review process:

\section{Racism vs. Race/Ethnic Discrimination}

Most of the existing scales deal with racism which is quite different from race/ethnicity-related perceived issues that is the focus of this study. Immigration issues like discrimination and perceptions of inequality in the workplace that needed to be captured in this study exist in multi-ethnic modern societies like New Zealand. Most scholars are in agreement that racial and ethnic categories are a social construction rather than biological fact (Butterfield, 2004). Race and ethnic discrimination are characterized by practices and actions of dominant race-ethnic groups that are directly or indirectly intended to deny subordinate race-ethnic groups certain rights (Feagin \& Eckberg, 1980).

The United Nations likewise do not make any distinction between racial and ethnic discrimination. Racism, on the other hand, denotes a lot of factors such as prejudice, violence, oppression, and is defined in varying forms. Racism has been defined as a set of pervasive actions of group inequality, and an ideology of racial domination that is characterized by the belief that subordinate racial group is biologically or culturally inferior (Bobo \& Fox, 2003). Hence, racism has a deeper and more intense sense of superiority over a particular "inferior" race that is rooted in historical oppression, while race/ethnic discrimination are actions that hinder other groups the same benefits of that of the majority. 
Popular scales that deal more with racism rather than race/ethnic discrimination like Racism and Life Experiences Scale - Personal and the Racism and Life Experiences Scale-Group (Harrel, 1997a; 1997b, in Rollins \& Valdez, 2006), and the Perceived Racism Scale (McNeilly et al., 1996) were regarded as not relevant for this study.

\section{Single Item Measures}

Other scales were found wanting on the number of items within scales, for instance just having single or double item scales. Frequently, there were only two items in scales used to measure discrimination and these were found to be inadequate to investigate a whole range of discriminatory perceptions in the workplace. Examples of these short scales are single-item measures (e.g. Levin et al. 2002) and 2-item measures (e.g. Barry \& Grilo, 2003; Romero \& Roberts, 1998; Shorey, et al., 2002). These small scales were found not to be appropriate for the current objective of this thesis.

\section{Workplace Related Scales}

Additionally, some of the scales that were reviewed look into discriminatory perceptions in the society in general, and do not directly focus on perceptions of organizational issues. Hence, the scale may have enough number of items but they were not related to workplace discrimination. Some scales have items that refer to workplace discrimination but also include general perceptions of discrimination related to race (e.g. Slaughter et al., 2005). A scale that is most useful for this study is the Sanchez and Brock (1996) Perceived Discrimination Against Hispanic Scale that they developed to measure perceived unfair practices in the workplace targeted at individuals 
of Hispanic-origin. However, this scale was not directly suitable because it was again directly applicable to a particular racial group - the Hispanics. Thus, the existing scales were not found directly adoptable for the current study.

\section{Immigrants' Perceptions}

Finally, the review on current measurements for discrimination particularly in the organizational setting did not result in finding one particular scale that could capture the themes from the focus groups discussion in Chapter 4. To reiterate, the themes found were based on the participants' discriminatory perceptions on formal and informal structures in the organization. For each structure, there were perceptions pertaining to jobentry and on-the-job experiences. The themes as illustrated in Figure 4.1 of Chapter 4 are the following: Quadrant 1 (formal structures-job entry) - the themes were language/accent, overseas qualifications not recognized and "kiwi" experience; Quadrant 2 (formal structures-on the job) - Quadrant 3 (informal structures-job entry) - reluctance towards coloured people, network/knowing people; and Quadrant 4 (informal structures-on the job) distrust for coloured people.

There is a need to probe discriminatory perceptions in the workplace as viewed by immigrants. A scale for migrants was developed by JasinskajaLahti \& Liebkind (2001) that measured experiences and frequency of being teased, threatened, being rejected due to one's ethnic background. However, this was not also found suitable for working immigrant sample in this thesis. A 4-item scale for immigrant was also developed by Jasinskaja-Lahti et al. (2007) measuring frequency of perceived ethnic discrimination at work. 
However, aside from minimal number of items, the scale again was found not directly suitable since the current study is not interested in measuring frequency of discrimination.

Frequency scales usually require respondents to answer yes or no and to count how frequent specific situations occur to them. Hence, frequency scales aim to sample a range of discriminatory events experienced by stigmatized individuals in different settings. Usually, these discriminatory events are pervasive to particular racial/ethnic groups specifically Blacks or African Americans who have a long history of oppression in different domains. This particular objective is deemed not appropriate for immigrant perceptions since discriminatory experiences of immigrants have different meanings for different ethnicities and are not captured by specific instances answerable by yes or no. The nature of perceived discrimination due to status (immigrant) or ethnicity is deemed more subtle and has not been established. Hence, the scale intended for the current research aimed to capture the subtleness and ambivalence that are inherent in immigrants' PD as depicted in the FGD themes of Chapter 4.

Summarized in Table 5.1 are the scales found during the review on measurements used in studies on perceptions of discrimination related to race or ethnicity and the evaluations regarding each scale. Thereby, considering the issues outlined above regarding existing PD scales, it was deemed appropriate to construct a new scale that would be most suitable to this study. Henceforth, the author proceeded with the development of a new set of PD scale using items coming from the themes of the focus groups discussions (as illustrated in Figure 4.1 of Chapter 4) as well as selected items from existing 
Table 5.1

A Sampling and Evaluation of Existing Scales for Measuring Race/Ethnicity-Related Perceptions of Discrimination

\begin{tabular}{|c|c|c|c|}
\hline Author & Scale Description & Type of Measurement & Evaluation \\
\hline Barry \& Grilo (2003) & 2 -item scales for personal and group perceptions & Personal vs. Group Perceptions & Minimal number of items \\
\hline Bourguignon, et al (2006) & 7-item scale for personal and group perceptions & Personal vs. Group Perceptions & Pertain more to racism issues \\
\hline Harrel (1997a; 1997b) & $\begin{array}{l}\text { Racism and Life Experiences Scale-Personal } \\
\text { (RaLES-PER) and the Racism and Life } \\
\text { Experiences Scale-Group (RaLES-GRP) }\end{array}$ & Personal vs. Group Perceptions & Pertain more to racism issues \\
\hline Levin, et al (2002) & single item scales for personal and group perceptions & Personal vs. Group Perceptions & Minimal number of items \\
\hline Romero \& Roberts (1998) & 2- item scales for personal and group perceptions & Personal vs. Group Perceptions & Minimal number of items \\
\hline $\begin{array}{l}\text { Spencer-Roger \& Collins } \\
(2006)\end{array}$ & $\begin{array}{l}\text { the Racial-Ethnic Group Disadvantage Scale } \\
\text { (REGDS) }\end{array}$ & Personal vs. Group Perceptions & Intended for single ethnic group (Latinos) \\
\hline Finch et al (2000) & $\begin{array}{l}3 \text { item scale for Mexican sample later on used by Lee } \\
\text { (2003) for American students of Indian descent }\end{array}$ & $\begin{array}{l}\text { Group Identification } \\
\text { (Race/Ethnicity) }\end{array}$ & Minimal number of items \\
\hline James et al. (1994) - & $\begin{array}{l}\text { Workplace Prejudice/ Discrimination Inventory } \\
\text { (WPDI) }\end{array}$ & $\begin{array}{l}\text { Group Identification } \\
\text { (Race/Ethnicity) }\end{array}$ & Minimal number of items \\
\hline $\begin{array}{l}\text { Jasinskaja-Lahti \& Liebkind } \\
\text { (2001) }\end{array}$ & $\begin{array}{l}\text { 9-item scale for Russian-speaking adolescent sample } \\
\text { in the Netherlands }\end{array}$ & $\begin{array}{l}\text { Group Identification } \\
\text { (Race/Ethnicity) }\end{array}$ & Not focused on workplace context \\
\hline Jefferson \& Caldwell (2002) & Discriminatory Scenarios Questionnaire (DSQ) & $\begin{array}{l}\text { Group Identification } \\
\text { (Race/Ethnicity) }\end{array}$ & Designed for African Americans \\
\hline Konrad \& Spitz (2003) & 3-item scale for White and "men of colour" & $\begin{array}{l}\text { Group Identification } \\
\text { (Race/Ethnicity) }\end{array}$ & Minimal number of items \\
\hline McConahay (1986) & Modern Racism Scale (MRS) & $\begin{array}{l}\text { Group Identification } \\
\text { (Race/Ethnicity) }\end{array}$ & $\begin{array}{l}\text { Pertain more to Black racism/discrimination } \\
\text { issues }\end{array}$ \\
\hline McWhirter (1997) & Perceived Occupational Barrier Scale ([POBS] & $\begin{array}{l}\text { Group Identification } \\
\text { (Race/Ethnicity) }\end{array}$ & $\begin{array}{l}\text { Designed for school setting and workplace } \\
\text { setting }\end{array}$ \\
\hline Sanchez \& Brock (1996) & a 10-item PD Against Hispanic Scale & $\begin{array}{l}\text { Group Identification } \\
\text { (Race/Ethnicity) }\end{array}$ & Designed for single ethnic group (Hispanic) \\
\hline Turner \& Turner’s (1975) & PD Against Blacks Scale & $\begin{array}{l}\text { Group Identification } \\
\text { (Race/Ethnicity) }\end{array}$ & $\begin{array}{l}\text { Pertain more to Black racism/discrimination } \\
\text { issues }\end{array}$ \\
\hline Verkuyten \& Brug (2002) & Adapted from Ruggiero \& Taylor (2001). & $\begin{array}{l}\text { Group Identification } \\
\text { (Race/Ethnicity) }\end{array}$ & \\
\hline
\end{tabular}


Table 5.1 (continued)

\begin{tabular}{|c|c|c|c|}
\hline Author & Scale Description & Type of Measurement & Evaluation \\
\hline Ensher et al (2001) & 12 item scale for employees of various ethnic groups & Lateral & $\begin{array}{l}\text { Minimal number of items measuring each } \\
\text { level of PD }\end{array}$ \\
\hline Konrad \& Spitz (2003) & $\begin{array}{l}\text { 3-item scale called Belief in Labour Market } \\
\text { Discrimination measuring systemic PD }\end{array}$ & Lateral & Minimal number of items \\
\hline Watts \& Carter (1991) & $\begin{array}{l}\text { Scale for Blacks from items developed by authors and } \\
\text { institutional-racism items from Barbarin \& Gilbert } \\
\text { (1981) }\end{array}$ & Lateral & $\begin{array}{l}\text { Pertain more to Black racism/discrimination } \\
\text { issues }\end{array}$ \\
\hline Way (1997) & 14-item scale measuring adult and peer perceptions & Lateral & Not focused on workplace context \\
\hline Cassidy et al (2005) & $\begin{array}{l}\text { PD scale based on items of Verkuyten (1998) \& } \\
\text { Phinney et al (1998) }\end{array}$ & Frequency & Not focused on workplace context \\
\hline Collado-Proctor (1999). & $\begin{array}{l}\text { Perceived Racism Scale for Latina/os (PRSL) } \\
\text { patterned from PRS. }\end{array}$ & Frequency & Designed for Latinos (single ethnic group) \\
\hline Deitch et al (2003) & Everyday Mistreatment Scale (EMS) & Frequency & $\begin{array}{l}\text { Pertain more to Black racism/discrimination } \\
\text { issues }\end{array}$ \\
\hline Jasinkaja et al (2007) & $\begin{array}{l}\text { 4-item scale for Russian- and Estonian speaking } \\
\text { immigrants in the Netherlands. }\end{array}$ & Frequency & Minimal number of items \\
\hline Landrine \& Klonoff, (1996) & $\begin{array}{l}\text { Schedule of Racist Events (SRE) for Black adults in } \\
\text { various contexts }\end{array}$ & Frequency & $\begin{array}{l}\text { Pertain more to Black racism/discrimination } \\
\text { issues }\end{array}$ \\
\hline McNeilly et al. (1996) & Perceived Racism Scale (PRS) for African Americans & Frequency & $\begin{array}{l}\text { Pertain more to Black racism/discrimination } \\
\text { issues }\end{array}$ \\
\hline Phinney et al (1998) & 7-item scale measuring frequency of PD & Frequency & Not focused on workplace context \\
\hline Rollins \& Valdez (2006) & $\begin{array}{l}\text { used Daily Life Experiences (DLE-R) which is a } \\
\text { composite of RaLES by Harrel (1997). The DLE-R } \\
\text { was renamed DLE-F by Scott \& House (2005) }\end{array}$ & Frequency & $\begin{array}{l}\text { Pertain more to Black racism/discrimination } \\
\text { issues }\end{array}$ \\
\hline Zlobina et al (2006) & 6-item PD for immigrants in Spain & Frequency & Not focused on workplace context \\
\hline
\end{tabular}


scales found from gender studies like the Organizational Stressor Scale (Beatty, 1996), Women Workplace Culture Questionnaire (Bergman, 2003; Bergman \& Hallberg, 2002) and race/ethnic PD studies like the Discrimination Scale by Martin et al. (2003) measuring current and lifetime discrimination, and Perceived Discrimination Scale for Latino/as (Sanchez \& Brock, 1995). The items from the existing scales were either adapted verbatim or modified to fit into the new scale.

In summary, the review of existing PD scales that could be adapted in the current thesis' smaller studies did not yield any one particular measure that is directly suitable for migrant perceptions. Thus, the next step taken was to construct a new scale appropriate for the current area of study. The succeeding section of this Chapter outlines the steps taken towards the development of the scale on immigrants' perceptions of discrimination relevant to the New Zealand workplace. This scale is intended to be used for the succeeding investigation of the thesis research questions.

\section{Methodology}

\section{Item Development}

After the review of existing PD measurements and the focus groups discussion, fifty-five (55) items that reflect the themes that emerged from the focus groups, from the literature review and existing scales (Beatty, 1996; Bergman, 2003; Bergman \& Hallberg, 2002; Martin et al., 2003; Sanchez \& Brock, 1995) were generated for the preliminary version. The initial classifications of the items were assigned to the following three main scales. (1) Job-Entry PD: this was composed of statements relating to perceptions that 
are relevant during the pre-hiring process such as physical characteristics, educational background, professional/work experience, language/accent, and networking/knowing people. (2) On-the-Job Formal PD (formal structures): this contained items pertaining to perceptions regarding promotions/trainings, pay, fringe benefits, overall organizational policies. (3) On-the-Job Informal PD (informal structures): this scale contained items describing perceptions regarding relational interactions with co-workers in different hierarchies (e.g., general, peers and superiors). Please refer to Appendix B for the full list of the initial 55 items. The three scales from hereon will be collectively referred to as the Immigrants' Perceptions of Discrimination in the Workplace Scales (IPDWS). Most of the items in the IPDWS are patterned from the themes in the focus groups. Table 5.2 is an illustration how the three scales of IPDWS fits in the focus groups discussion framework.

Table 5.2

IPDWS' Fit in the FGD Model

\begin{tabular}{lcc}
\hline & \multicolumn{2}{c}{ Phases of Employment } \\
\cline { 2 - 3 } Formal Structures & $\begin{array}{c}\text { Jinding Work- } \text { - e.g. Recognition of } \\
\text { Education \& Work Experience, } \\
\text { Local Requirements }\end{array}$ & On-the-Job \\
\hline Informal Structures & Finding Work - e.g. Networking items & Work Relationships \\
\hline * IPDWS - Immigrant Discrimination in the Workplace Scales, FGD - Focus Groups \\
Discussion
\end{tabular}

Subsequently, the 55 items (without initial categorizations) were presented to a group of 18 postgraduate students and lecturers that belonged to a cross-cultural research centre in the university. The ethnic backgrounds of 
the group members were diverse (e.g. New Zealanders, Germans, Estonian, Swedish, Chinese, American, and French). The whole group were divided into three subgroups and were asked to (a) group the items to an appropriate classification as they see fit, (b) provide a category name for the classification, and (c) rank the individual items according to their representativeness to the classification they have chosen. Thus, the more the item represented the classification, the higher the ranking assigned to it.

The resulting classifications from the group activity provided validation of my initial categorization of the items, though moderately differing in classification names. Illustrated in Table 5.3 are the groups' output vis a vis my initial classification. The three groups' categorizations of the items resemble my categorization for the items related to personal characteristics, recognition of qualifications, language, network, organizational policies and work relationships.

Among the three groups, Group Three's two main classifications were identical with my headings of the major classifications (i.e. job entry/getting a job and on-the-job) although they did not subdivide the "on-the-job" category. Groups One and Two did not name any major classifications to their categorizations. However, the categories for the items also matched my sorting of items. They sorted the items into categories related to individual characteristics such as recognition of qualifications and language, formal structure categories such as promotion and compensation, and the relationships in organizations.

Consequently, after further discussions with the group members about the phrasing of the items, some items were modified or edited for clarity and 
new items were generated. Thereby, a total of 67 items (see Appendix C) were included in the pilot version of the IPDWS.

Table 5.3

Results of the Content Validation of Initial Scale Items

\begin{tabular}{|c|c|c|c|}
\hline Author & Group 1 & Group 2 & Group 3 \\
\hline $\begin{array}{l}\text { Job Entry } \\
\text { Discrimination } \\
\text { - Personal } \\
\text { characteristics } \\
\text { - Educational } \\
\text { background } \\
\text { - Professional/work } \\
\text { experience } \\
\text { - Language accent } \\
\text { - Networking/knowing } \\
\text { people }\end{array}$ & $\begin{array}{l}\text { Discrimination/stereotyping } \\
\text { Recognition of } \\
\quad \text { work/education } \\
\text { Recognition of background } \\
\text { Language discrimination } \\
\text { Network }\end{array}$ & $\begin{array}{l}\text { Discrimination } \\
\quad \text { (individual } \\
\quad \text { characteristics) } \\
\text { Qualifications } \\
\text { Access to jobs }\end{array}$ & $\begin{array}{l}\text { Getting a job } \\
\text { - accent plus looks } \\
\text { (ethnic barriers) } \\
\text { - previous work or } \\
\text { educational } \\
\text { experience } \\
\text { - networking }\end{array}$ \\
\hline $\begin{array}{l}\text { On-the-Job PD } \\
\text { (Formal Structures) } \\
\text { - Promotions/ } \\
\quad \text { developmental } \\
\text { - Salary/pay } \\
\text { - Fringe benefits } \\
\text { - Overall }\end{array}$ & $\begin{array}{l}\text { Organizational Policies } \\
\text { Financial aspect (career } \\
\quad \text { advancement) } \\
\text { Financial aspect (salary) } \\
\text { Financial Aspect } \\
\quad \text { (entitlements) }\end{array}$ & $\begin{array}{l}\text { Organizational } \\
\quad \text { policies } \\
\text { Career opportunities } \\
\text { Equity }\end{array}$ & $\begin{array}{l}\text { On-the-job } \\
\text { - organizational } \\
\text { policies } \\
\text { - general promotion } \\
\text { (not culture-based) } \\
\text { - salary } \\
\text { - work benefits }\end{array}$ \\
\hline $\begin{array}{l}\text { On-the-Job PD } \\
\quad \text { (Informal } \\
\text { Structures) } \\
\text { - General } \\
\text { - Peers } \\
\text { - Supervisors }\end{array}$ & Quality of work relationship & $\begin{array}{l}\text { Interpersonal } \\
\text { relationships }\end{array}$ & $\begin{array}{l}\text {-relationship with } \\
\text { superiors } \\
\text { - co-worker inclusion- } \\
\text { exclusion }\end{array}$ \\
\hline
\end{tabular}

\section{Instrument Refinement and Initial Psychometric Properties}

The IPDWS-pilot versions (the original version) were tested on a diverse sample of working immigrants to determine the factor structures of each PD scale. The overall aim was to develop a psychometrically valid measure of PD in the workplace as perceived by immigrants. There were two sub-objectives in this section. The first objective was the horizontal investigation of the PD construct by exploring the factor structures and 
examining the reliability and construct validity of the scales ${ }^{3}$. This included data reduction or reducing the original number of items through the deletion of poorly performing items. Construct validity of the resulting factor structures was examined in terms of its concurrent validity with English language proficiency which has been found to be related to PD (Barry \& Grilo, 2003, Goto, Gee \& Takuchi, 2002; Romero \& Roberts, 1998). The second objective was the vertical investigation of PD's factor structures. This was achieved by conducting additional analysis to determine if the primary factors obtained from the initial principal components analysis will converge to form higher order factors. The presence of higher order factors broadens the understanding of the nature of PD process.

\section{Method}

Participants.

Respondents were a diverse group of employed immigrants in different organizations in Wellington City, the capital city of New Zealand. From the 400 questionnaires initially distributed 155 were returned, resulting in a response rate of $38.8 \%$. Thus, the participant sample was a total of 155 firstgeneration immigrants (female 52\%, male $46 \%$, and $2 \%$ did not state their gender). Mean age was 40 years old $(M=39.55 ; S D=8.97)$ with an average length of stay in New Zealand of 7.9 years. In terms of immigrant status, $49 \%$ had already obtained their New Zealand citizenship, 43\% were permanent residents, $6 \%$ were on work visa/permit and $2 \%$ opted not to answer the question.

3 The terminologies "horizontal" and "vertical" investigation of the PD factor structure was patterned from the investigation of horizontal ( 1 st order factors $)$ and vertical $\left(2^{\text {nd }}\right.$ order factors) structure of Environmental Attitudes (Heberlein, 1981, in Milfont, 2007). 
The participants were occupying the positions of chief executive/general managers/legislators $(1.3 \%)$, middle managers/supervisors (11\%), professional $(53.5 \%)$, clerical and administrative workers $(22.6 \%)$, community and personal service workers $(2.6 \%)$, technicians and trade workers $(1.9 \%)$, sales workers $(2.6 \%)$, labourers $(2.6 \%)$, other positions not mentioned above $(1.3 \%)$, and $0.6 \%$ opted not to indicate the position levels they were occupying. Majority of the respondents were in full-time work (91\%). Their tenures in their current organizations ranged from one month to 30 years, with a mean of 3.11 years. Table 5.4 presents the demographic characteristics of the sample.

Respondents' Workplace. The respondents worked in organizations belonging to different industries (retail trade $16.8 \%$, business and financial services $14.2 \%$, education $9 \%$, accommodation, cafes and restaurant businesses $9 \%$, government administration and defence $8.4 \%$, health and communication services $7.7 \%$, others $11 . \%$. See table 5.5 for the complete list of industries identified by respondents. Most of these organizations were privately owned $(53.5 \%)$ including six non-government organizations. When participants were asked to rate how diverse their organization are in terms of ethnicity and nationality, from a 7 -point scale $(1=$ not diverse at all, $7=$ very diverse) the responses had a mean of 5.3 which indicated that organizations were quite diverse. 
Table 5.4

Participants' Demographics ( $=155)$

\begin{tabular}{lcc}
\hline & $\mathrm{N}$ & \\
& & \\
\hline & 155 & \\
Total No. of Participants & 80 & $52 \%$ \\
Female & & \\
Age Range & 25 & $16.1 \%$ \\
30 \& less & 37 & $23.9 \%$ \\
31-40 & 50 & $32.3 \%$ \\
41-50 & 8 & $5.2 \%$ \\
51-60 & 3 & $1.9 \%$ \\
above 60 & 32 & $20.6 \%$ \\
- Missing Data & & \\
Highest Completed Qualifications & 5 & $3.2 \%$ \\
- secondary school & 13 & $8.4 \%$ \\
- technical/diploma & 92 & $59.4 \%$ \\
- bachelor/tertiary & 11 & $7.1 \%$ \\
- postgraduate/honours & 28 & $18.1 \%$ \\
- masters/phd \& above & 6 & $3.9 \%$ \\
- Missing Data/other & & \\
Job Levels & 2 & $1.3 \%$ \\
- Chief Exec/Gen Managers & 17 & $11.0 \%$ \\
- Middle Managers/Supervisors & 83 & $54.5 \%$ \\
- Professional & 3 & $1.9 \%$ \\
- Technicians/Trade Workers & 4 & $2.6 \%$ \\
- Community/Personal Svc Worker & 35 & $22.6 \%$ \\
- Clerical/Admin Worker & 4 & $2.6 \%$ \\
- Sales Worker & 4 & $2.6 \%$ \\
- Labourer & 3 & $1.9 \%$ \\
- Missing Data/Other & & \\
Citizenship Status & 76 & $49.0 \%$ \\
- NZ citizens & 66 & $42.6 \%$ \\
- NZ permanent residents & 10 & $6.5 \%$ \\
- Work Visa/Permit & 3 & $1.9 \%$ \\
- Missing Data & \\
\hline
\end{tabular}


Table 5.5

Organizations' Demographics

\begin{tabular}{|c|c|c|}
\hline & $\mathrm{N}$ & $\%$ \\
\hline \multicolumn{3}{|l|}{ Industry of the Organization } \\
\hline Accommodation, Cafes \& Restaurants & 14 & $9 \%$ \\
\hline Agriculture, Forestry \& Fishing & 3 & $1.9 \%$ \\
\hline Business \& Financial Services & 22 & $14.2 \%$ \\
\hline Communication Services & 5 & $3.2 \%$ \\
\hline Construction & 5 & $3.2 \%$ \\
\hline Education & 14 & $9 \%$ \\
\hline Electricity, Gas and Water Supply & 2 & $1.3 \%$ \\
\hline Finance and Insurance & 7 & $4.5 \%$ \\
\hline Government, Administration and Defense & 13 & $8.4 \%$ \\
\hline Health \& Community Services & 12 & $7.7 \%$ \\
\hline Manufacturing & 5 & $3.2 \%$ \\
\hline Personal and other services & 1 & $0.6 \%$ \\
\hline Property and Business Services & 1 & $0.6 \%$ \\
\hline Retail Trade & 26 & $16.8 \%$ \\
\hline Transport \& Storage & 4 & $2.6 \%$ \\
\hline Wholesale Trade & 4 & $2.6 \%$ \\
\hline Others & 17 & $11 \%$ \\
\hline Total & 155 & $100 \%$ \\
\hline \multicolumn{3}{|l|}{ Sector of the Organization } \\
\hline - Private & 83 & $53.5 \%$ \\
\hline - Public & 64 & $41.3 \%$ \\
\hline - NGO & 6 & $3.9 \%$ \\
\hline - Missing Data/Others & 2 & $1.3 \%$ \\
\hline Total & 155 & $100 \%$ \\
\hline
\end{tabular}

Respondents reported their ethnic background as Asians (70\%), Europeans (10\%), South Africans (5\%), Pacific Peoples (4\%), Middle Eastern (3\%), Latin American (1\%), Others (1\%), and 5\% opted not to answer the question. Those who were born in Asia were further identified as Filipinos (58), Indians (21), Chinese (14), Sri Lankan (4), Vietnamese (2), Cambodian (2), Indonesian (1), Malaysian Chinese (1), Japanese (1), and more generalized descriptions as a Southeast Asian (1) and Asian (2). Please see Table 5.6 for a detailed tabulation of ethnic backgrounds. 
Table 5.6

Participants' Ethnic Background

\begin{tabular}{lcc}
\hline & $\mathrm{N}$ & $\%$ \\
\hline Total No. of Participants & 155 & \\
Breakdown of Self-Reported Ethnicities & & \\
European & 16 & $10.3 \%$ \\
South African & 8 & $5.2 \%$ \\
Pacific People & 6 & $3.9 \%$ \\
Asian & 109 & $70.3 \%$ \\
Latin America & 2 & $1.3 \%$ \\
Middle Eastern & 5 & $3.2 \%$ \\
New Zealander & 1 & $0.6 \%$ \\
Missing Data/Other & 8 & $5.1 \%$ \\
& & \\
Breakdown of Asians & 2 & $1.9 \%$ \\
Asian nfd* & 1 & $0.9 \%$ \\
Southeast Asian nfd & 58 & $53.7 \%$ \\
Filipino & 2 & $1.9 \%$ \\
Cambodian & 2 & $1.9 \%$ \\
Vietnamese & 1 & $0.9 \%$ \\
Indonesian & 14 & $13.0 \%$ \\
Chinese nfd & 1 & $0.9 \%$ \\
Malaysian Chinese & 23 & $21.3 \%$ \\
Indian nfd & 4 & $3.7 \%$ \\
Sri Lankan nfd & & \\
& & \\
\hline
\end{tabular}

*nfd - not further defined

Instrument.

(IPDWS-P). The Immigrants Perceptions of Discrimination in the Workplace Scales (IPDWS-Pilot version) are composed of three main scales (see Appendix C). There were 67 items in total distributed to the first scale "finding work" (24 items), the second scale "working conditions" (28 items) and the third scale "work relationships" (15 items). The first scale, Finding Work pertains to $\mathrm{PD}$ in relation to how people are hired in their organization according to certain practices or company regulations (e.g. "My accent is a barrier to obtaining employment"). Responses are recorded on a 5-point Likert 
scale ranging from 1 (strongly agree) to 5 (strongly disagree) with low scores indicating higher perception of discrimination.

The second scale Working Conditions measures perceived discriminatory practices or policies affecting employees in terms of compensation, benefits, training and career progression (e.g. "My opportunity for advancement is somewhat limited"). It had an item response format of 5point scale similar to finding work, ranging from 1 (strongly agree) to 5 (strongly disagree) with low scores indicating higher PD.

Lastly, Work Relationships measures discriminatory perceptions on interactions and relationships with workers and superiors within the working environment (e.g. “At work, my supervisor has negative stereotypes about my culture or ethnic group and treats me as if they were true"). The item response format was on a scale from 1 (All the time) to 5 (Never) with high scores indicating lower perception of discrimination. Before further statistical analysis were done, composites of PD for each scale were computed by reverse scoring all items such that higher scores indicate greater levels of perceptions of discrimination.

Control Variables. Included in the questionnaires are questions related to personal and demographic information such as age, gender, citizenship/nationality ethnicity, country of birth, highest educational qualification, current position title, tenure, position level, job status (fulltime/part-time), income, organization's sector, size and industry. Question format and responses (categories/subcategories) were mostly patterned from the New Zealand Statistics Survey (2002). 
The questionnaire also included a self-rated English language proficiency scale containing three questions related to writing (Written English Competency), speaking (Spoken English Fluency) and comprehension of the English language (English Comprehension). This was intended to measure the participants' personal assessment of their level of Englishlanguage proficiency on a 5-point scale, ratings of 1 (excellent) to 5 (can't write/speak/understand English). Scores obtained on the three items were calculated as a single composite score for the immigrant sample $(\alpha=.94)$.

\section{Procedure.}

The data gathering was conducted from November 2006 to February 2007. I networked with migrant community leaders in Wellington City for the distribution of questionnaires. Questionnaires were also distributed in organizations through contact persons and through snowball sampling. Further, I also attended ethnic forums or social events and distributed questionnaires after the sessions. Distributions were also done after mass in local churches during weekends and in the streets during week days in the business district of the city.

The survey pack included an envelope with the anonymous questionnaire, a pre-stamped return envelope (for the completed questionnaire), and separate forms requesting a contact address with a prestamped return envelope for if the respondents wanted copies of the research findings. This way the questionnaire could not be linked back to the participant if they opted to receive the research findings. The questionnaire was estimated to take $30-45$ minutes to complete. The respondents filled out the questionnaires at their own pace and wherever they wanted. Once the 
questionnaires were completed, the questionnaires were either posted back to me or Dr. Ronald Fischer, or were given back to the contact persons in sealed envelopes and were picked up on a mutually agreed schedule. Requests for copies of the research findings were mailed separately if questionnaires were handed back to the contact persons.

\section{Data Analyses.}

All data analyses were conducted using the SPSS statistical package.

\section{Results}

\section{Exploratory factor analysis - First order factors.}

The first step taken was to conduct a principal component analysis (PCA) using oblique rotation (Direct Oblimin with Kaiser normalization) for each of the three main scales of IPDWS. Oblique rotation was used due to the assumption that the components are intercorrelated. A number of guidelines were observed in determining the number of factors to be extracted namely, 1) Kaiser's (1970) greater-than-one rule, 2) inspection of the scree plot, 3) retention of items with loadings of .50 or better, and 4) for an item that shows cross loadings on two factors, if the difference of loadings is less than .10 then the item is dropped. Inspection of the scree plot (Kim \& Mueller, 1978) and the pattern matrix suggested four interpretable factors accounting for $54.51 \%$ of the variance for the first scale (finding work), three interpretable factors with a variance of $49.06 \%$ for the second scale (working conditions), and a three factor structure (with a variance of $68.46 \%$ ) for the third scale (work relationship).

Finding Work. Initial extraction generated 5 eigenvalues greater than 1 but after examination of the scree plot and pattern matrix, only 4 
interpretable factors were eventually considered. Based on item content, the four factors in Finding Work were named non-local (9 items), nonrecognition of education and experience (5 items), networking (3 items) and need for local requirements (4 items).

The first factor (non-local) pertains to the individual's perceptions of his/her personal characteristics as the basis for the perceptions of discrimination (e.g. "My accent is a barrier to obtaining employment"). All items in non-local loaded from .54 to .85 (mean loading $=.68$ ). The second factor (non-recognition of education and experience) pertains to perceptions that local authorities do not acknowledge the education and work experience they obtained outside of New Zealand (e.g. "My educational qualifications gained overseas were recognized in my job" - recoded as a negatively worded item). The factors for non-recognition of education and experience loaded from .70 to .86 (mean loading $=.79$ ). The third factor, networking, describes the importance of having contact persons in organization in order to be considered for a job in organizations (e.g. "It is easy to get a job in New Zealand if you know people in an organization"). The items for networking loaded from .68 to .82 (mean loading $=.76$ ). Lastly, local requirements refers to perceptions that local authorities only prefer qualifications and experience specific to New Zealand. (e.g. "I need to obtain local qualifications to obtain the job that I want"). Items loaded from .50 to .72 (mean loading $=.59$ ). Presented in Table 5.7 are the 21 items of the scale Finding Work with the pattern matrix's factor loadings, eigenvalues and the cumulative variance explained. 
Table 5.7

Factor Loadings and Internal Consistency for Finding Work

\begin{tabular}{|c|c|c|c|c|}
\hline \multirow[b]{2}{*}{ Items } & \multicolumn{4}{|c|}{ Factors } \\
\hline & 1 & 2 & 3 & 4 \\
\hline \multicolumn{5}{|l|}{ Factor 1: Non-Local } \\
\hline 7. I was denied a job because I am not a native English Speaker. & .85 & -.02 & .01 & -.08 \\
\hline 4. I was denied a job because of my looks. & .81 & .07 & -.17 & -.05 \\
\hline 13. My accent is a barrier to obtaining employment. & .77 & -.02 & -.00 & .14 \\
\hline 17. I was denied a desired position due to my accent. & .76 & -.06 & -.07 & .14 \\
\hline $\begin{array}{l}\text { 22. Another applicant with fewer qualifications got the job because my } \\
\text { looks are different from majority of the workforce. }\end{array}$ & .70 & -.06 & -.08 & .09 \\
\hline $\begin{array}{l}\text { 15. Having English as a second language is a barrier to enter the } \\
\text { workforce }\end{array}$ & .60 & -.04 & -.05 & .12 \\
\hline 9. My organization prefers to hire local New Zealanders. & .59 & .11 & .18 & -.02 \\
\hline 10. English-native speakers have better access to jobs. & .54 & .05 & .31 & .13 \\
\hline 1. My name is a hindrance in getting a job. & .54 & -.25 & .08 & -.26 \\
\hline 11. I didn't get a job because I lack work experience* & .47 & -.20 & .19 & .21 \\
\hline \multicolumn{5}{|l|}{$\begin{array}{l}\text { Factor 2: Non-Recognition of Education/Experience } \\
2 . \quad \text { My educational qualifications gained overseas were recognized in } \\
\text { my job. }\end{array}$} \\
\hline $\begin{array}{l}\text { 5. My educational background is recognized in my current position. } \\
\text { 21. I am employed according to my professional/work experience }\end{array}$ & .05 & 0.85 & -.07 & .08 \\
\hline gained overseas. & .09 & 0.77 & .11 & -.18 \\
\hline $\begin{array}{l}\text { 8. I was hired according to my educational qualifications. } \\
\text { 14. My skills gained from previous work experience overseas are }\end{array}$ & -.04 & 0.75 & -.12 & .09 \\
\hline utilized in my job. & -.01 & 0.70 & .12 & -.05 \\
\hline $\begin{array}{l}\text { 3. Recruitment consultant/agencies are helpful in finding a job for } \\
\text { people of my ethnic background.* }\end{array}$ & -.25 & .46 & .02 & .11 \\
\hline $\begin{array}{l}\text { Factor 3: Networking } \\
6 . \quad \text { It is easy to get a job in New Zealand if you know people in an } \\
\text { organization. }\end{array}$ & .09 & -.02 & 0.82 & -.25 \\
\hline $\begin{array}{l}\text { 20. Contact persons in an organization can help you obtain a job. } \\
\text { 12. Networking is helpful in obtaining a job in New Zealand. }\end{array}$ & $\begin{array}{l}-.00 \\
-.07\end{array}$ & $\begin{array}{l}-.05 \\
.03\end{array}$ & $\begin{array}{l}0.77 \\
0.68\end{array}$ & $\begin{array}{l}.10 \\
.17\end{array}$ \\
\hline Factor 4: Local Requirements & & & & \\
\hline 18. I need to obtain local qualifications to obtain the job that I want. & .17 & -.10 & -.05 & 0.72 \\
\hline $\begin{array}{l}\text { 24. References are a necessity in finding a job in New Zealand. } \\
\text { 23. My educational training and skills are not recognized by NZ }\end{array}$ & -.10 & .10 & .20 & 0.60 \\
\hline $\begin{array}{l}\text { authorities. } \\
\text { 19. I got a job different from my previous work experience gained }\end{array}$ & .16 & -.23 & -.03 & 0.53 \\
\hline overseas. & .06 & -.38 & .06 & 0.50 \\
\hline $\begin{array}{l}\text { 16. I got a job relevant to my work experience but low in terms of } \\
\text { position level.* }\end{array}$ & 30 & .09 & -.06 & 0.47 \\
\hline Eigenvalues & 6.87 & 2.70 & 1.95 & 1.57 \\
\hline$\%$ of Variance of Unrotated Factor & 28.64 & 11.24 & 8.11 & 6.53 \\
\hline Cumulative variance (\%) & 28.64 & 39.87 & 47.98 & 54.51 \\
\hline Cronbach Alpha & .88 & .86 & .67 & .66 \\
\hline
\end{tabular}

Note: items included in the factor are written in boldface; * item deleted due to low factor loadings 
Work Conditions. Initial extraction generated 7 eigenvalues greater than 1. However, examination of items in pattern matrix suggested 3 interpretable solutions accounting for $49.06 \%$ of the variance.

The three factor solution in the Work Conditions Scale was named hindered career path and unequal opportunity (14 items), inadequate compensation (8) and differing treatment (2 items). Hindered career path and unequal opportunity pertains to discriminatory perceptions that hinder the career advancement of the individual and the perception that procedures and policies are discriminatory due to group membership (e.g. "I have a good chance for promotion" - recoded as a negatively worded item). Items in the first factor have loadings from .52 to .75 (mean loading $=.65$ ). The second factor is inadequate compensation which is composed of items that describe the perceptions of not being paid fairly according to comparable levels within the organization or within similar industries (e.g. "My salary/wage is not commensurate to the amount of work I do in this organization”). This second factor had item loadings from .54 to .72 (mean loading $=.64$ ). Differing treatment, the third factor, reflects perceptions that organizational policies or procedures are discriminatory to some groups (e.g. "This organization's policies and procedures are unfair to people of my ethnic background). The items in this last factor loaded from .53 to .55 (mean loading $=.54$ ). Table 5.8 shows the 24 items of Work Conditions with the factor loadings based on the pattern matrix, eigenvalues and their initial variance, and the cumulative variance explained. 
Table 5.8

Factor Loadings and Internal Consistency for Work Conditions

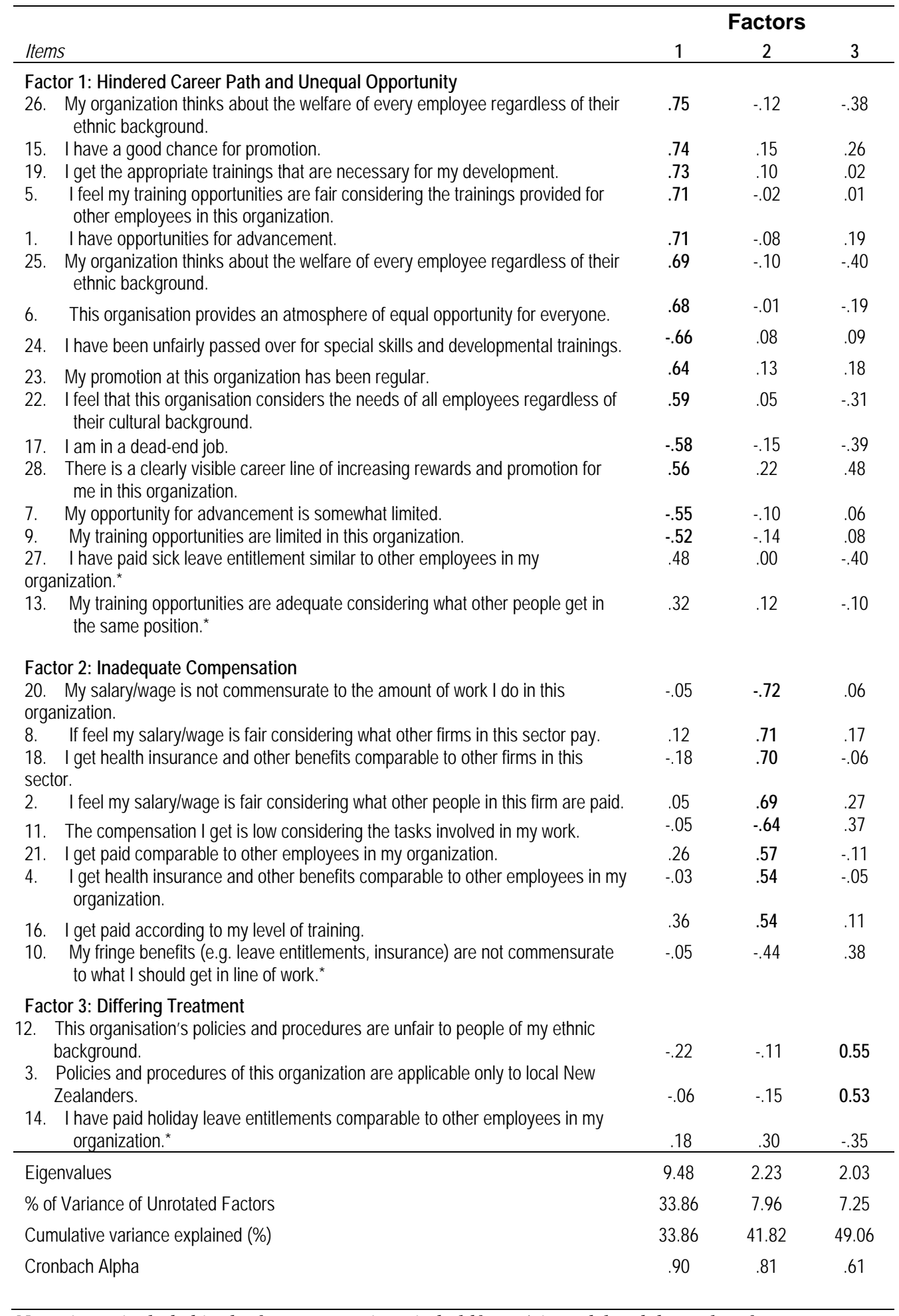

Note: items included in the factor are written in boldface; * item deleted due to low factor loadings 
Work Relationships. Initial extraction resulted in 3 eigenvalues higher than 1. After examining the pattern matrix, there were 13 items retained for the PD scale on Work Relationships which were distributed to the three factors named exclusion (5 items), derogation (3 items) and disharmonious work dynamics (5 items).

The first factor, exclusion, contains items pertaining to perceptions of being excluded due to group membership (e.g. "At work I feel that others exclude me from their activities because of my ethnic background"). It had item loadings from .61 to .93 (mean loading $=.75$ ).

The second factor is labelled derogation because these are perceptions about other people making fun of those who belong to other groups (e.g. "At work, I feel uncomfortable when others make jokes or negative commentaries about people of my ethnic background"). This second factor has item loadings from .84 to 93 (mean loading $=.90$ ).

The last factor in this scale is disharmonious work dynamics which pertains to perceptions that the quality of work-related interactions is dependent on group membership (e.g. "I have excellent working relationships with supervisors regardless of my ethnic background" - recoded as negatively worded items. It has item loadings from .56 to .93 (mean loading $=.78$ ).

Presented in Table 5.9 are the 15 items of Work Relationships with the pattern matrix's factor loadings, eigenvalues, their initial variance and cumulative variance explained. 
Table 5.9

Factor Loadings and Internal Consistency for Work Relationships

\begin{tabular}{|c|c|c|c|}
\hline \multirow{2}{*}{ Items } & \multicolumn{3}{|c|}{ Factors } \\
\hline & 1 & 2 & 3 \\
\hline \multicolumn{4}{|l|}{ Factor 1: Exclusion } \\
\hline $\begin{array}{l}\text { 10. At work, I feel that others exclude me from their activities because of } \\
\text { my ethnic background. }\end{array}$ & 0.94 & -.06 & .08 \\
\hline $\begin{array}{l}\text { 5. At work, I feel that my co-workers exclude me from their activities } \\
\text { because of my ethnic background. }\end{array}$ & 0.91 & -.08 & .02 \\
\hline $\begin{array}{l}\text { 13. At work, many people have negative stereotypes about my culture } \\
\text { or ethnic group and treat me as if they were true. }\end{array}$ & 0.64 & .19 & -.07 \\
\hline $\begin{array}{l}\text { 8. I experience frequent conflict with my co-workers on the job } \\
\text { because I come from a different culture. }\end{array}$ & 0.63 & .14 & -.09 \\
\hline $\begin{array}{l}\text { 3. At work, my supervisor has negative stereotypes about my culture } \\
\text { or ethnic group and treats me as if they were true. }\end{array}$ & 0.61 & .05 & -.16 \\
\hline $\begin{array}{l}\text { 15. I experience frequent conflict with my supervisor in my organization } \\
\text { because I come from a different culture.* }\end{array}$ & .42 & .38 & -.12 \\
\hline $\begin{array}{l}\text { 2. I have excellent working relationships with co-workers regardless of } \\
\text { our cultural backgrounds.* }\end{array}$ & -.39 & -.01 & .38 \\
\hline \multicolumn{4}{|l|}{ Factor 2: Derogation } \\
\hline $\begin{array}{l}\text { 1. At work, I feel uncomfortable when others make jokes or negative } \\
\text { commentaries about people of my ethnic background. }\end{array}$ & -.11 & .93 & -.10 \\
\hline $\begin{array}{l}\text { 14. At work, I feel uncomfortable when my co-workers make joke or } \\
\text { negative commentaries about my ethnic background. }\end{array}$ & .00 & .93 & .04 \\
\hline $\begin{array}{l}\text { 6. At work, I feel uncomfortable when my supervisor makes jokes or } \\
\text { negative commentaries about people of my ethnic background. }\end{array}$ & .10 & .84 & .11 \\
\hline \multicolumn{4}{|l|}{ Factor 3: Disharmonious Work Dynamics } \\
\hline $\begin{array}{l}\text { 9. My contributions at work are recognized by my supervisor even if I } \\
\text { come from a different cultural background. }\end{array}$ & .07 & -.02 & .93 \\
\hline $\begin{array}{l}\text { 4. My contributions at work are recognized by anyone although I am } \\
\text { different from them. }\end{array}$ & .10 & .05 & .90 \\
\hline $\begin{array}{l}\text { 12. My contributions at work are recognized by my co-workers although } \\
\text { I am different from them. }\end{array}$ & -.07 & .06 & .87 \\
\hline $\begin{array}{l}\text { 11. I have excellent working relationships with supervisors regardless of } \\
\text { my ethnic background. }\end{array}$ & -.20 & -.12 & .62 \\
\hline $\begin{array}{l}\text { 7. I have excellent working relationships with everyone regardless of } \\
\text { our cultural background. }\end{array}$ & -.25 & -.09 & .56 \\
\hline Eigenvalues & 7.22 & 2.00 & 1.06 \\
\hline$\%$ of Variance & 48.10 & 13.32 & 7.04 \\
\hline Cumulative variance explained (\%) & 48.10 & 61.42 & 68.46 \\
\hline Cronbach Alpha & .87 & .89 & .89 \\
\hline
\end{tabular}

Note: items included in the factor are written in boldface; * item deleted due to low factor loadings 


\section{Internal consistency.}

Internal reliability analyses were conducted for all factors. Almost all internal consistency values (Cronbach alphas) exceeded the minimum acceptable alpha coefficient levels of .70 as suggested by Nunnally and Bernstein (1994), except for networking ( $\alpha=.67)$, local requirements $(\alpha=.67)$ and differing treatment $(\alpha=.61)$. This may be due to the minimal number of items (2-4 items) although they have acceptable factor loadings (Cortina, 1993). Hence, the three factors were still included in the analysis since the factor loadings are $>.40$ (Guadagnoli \& Velicer, 1988). Further, the acceptable lower limit for Cronbach alpha can be reduced from .70 to .60 if the research is exploratory (Hair, Anderson, Tatham, \& Black, 1998).

\section{Intercorrelations.}

The significant correlations among factors from the three scales ranged from small to moderate (0.16 to 0.65$)$. The Local Requirements and Networking subscales (from Scale 1) did not result in significant correlations with the three factors of Scale 3 namely Exclusion, Derogation and Disharmonious Work Dynamics. Further, Networking also showed nonsignificant correlations with Non-Recognition of Educ and Experience (Scale 1) and Hindered Career Path/Unequal Opportunity (Scale 2). All factors in the three scales resulted in positive correlations except for Networking which showed significant negative correlation with Differing Treatment (from Scale 2) and non-significant negative relationships with Hindered Career Path/Unequal Opportunity (from Scale 2), Exclusion (from Scale 3) and Disharmonious Work Dynamics (from Scale 3). Factor intercorrelations (based on raw score scales) for the whole sample are presented in Table 5.10. 
Table 5.10

Zero-Order Correlations Among Factors in the IPDWS (Raw Score)

\begin{tabular}{|c|c|c|c|c|c|c|c|c|c|c|}
\hline & 1 & 2 & 3 & 4 & 5 & 6 & 7 & 8 & 9 & 10 \\
\hline \multicolumn{11}{|l|}{ Scale 1: Finding Work } \\
\hline 1. Non-Local & - & $0.36^{* *}$ & $0.45^{* *}$ & $0.17^{*}$ & $0.40^{* *}$ & $0.42^{* *}$ & $0.32^{* *}$ & $0.44^{* *}$ & $0.25^{* *}$ & $0.43^{* *}$ \\
\hline 2. Non-Recog of Educ/Experience & & - & $0.39^{* *}$ & 0.00 & $0.38^{* *}$ & $0.16^{*}$ & $0.40^{* *}$ & $0.19^{*}$ & $0.21^{* *}$ & $0.16^{*}$ \\
\hline 3. Local Requirements & & & - & $0.17^{*}$ & $0.16^{*}$ & $0.19^{*}$ & $0.28^{\star *}$ & 0.15 & 0.14 & 0.14 \\
\hline 4. Networking & & & & - & -0.01 & $-0.17^{*}$ & $-0.26^{* *}$ & -0.08 & 0.00 & -0.13 \\
\hline \multicolumn{11}{|l|}{$\begin{array}{l}\text { Scale 2: Work Conditions } \\
\text { 5. Hindered Careerpath \& Unequal }\end{array}$} \\
\hline Opportunity & & & & & - & $0.38^{* *}$ & $0.60^{* *}$ & $0.43^{* *}$ & $0.24^{* *}$ & $0.58^{* *}$ \\
\hline 6. Differing Treatment & & & & & & - & $0.32^{\star *}$ & $0.52^{* *}$ & $0.30^{\star *}$ & $0.50^{* *}$ \\
\hline 7. Indequate Compensation & & & & & & & - & $0.32^{* *}$ & $0.17^{*}$ & $0.38^{* *}$ \\
\hline \multicolumn{11}{|l|}{ Scale 3: Work Relationships } \\
\hline 8. Exclusion & & & & & & & & - & $0.32^{\star *}$ & $0.65^{\star *}$ \\
\hline 9. Derogation & & & & & & & & & - & $0.29^{* *}$ \\
\hline 10. Disharmonious Work dynamics & & & & & & & & & & - \\
\hline
\end{tabular}

$* \mathrm{p}<.05 ; * * \mathrm{p}<.01$ (two -tailed)

Note: IPDWS - Immigrant' Perception of Discrimination in the Workplace Scales 


\section{Validation.}

Language is a handicap for immigrants that can result in frustration and powerlessness (Cheung \& Dobkin de Rios, 1982). Being less educated may mean poor English language skills for immigrants requiring the need for an interpreter while those who are have proficient language skills may still be "misunderstood" due to their accent. For instance, American-accented speakers are perceived to be more attractive than Chinese-accented speakers (Cargile, 1997).

In acculturation studies, it has been found that less English language proficiency may be related to higher perceptions of discrimination (Romero \& Roberts, 1998). Barry and Grilo (2003) investigated personal and group perceived discrimination of East Asian immigrants in the United States and found that participants with greater English proficiency report less perceived personal discrimination. In another study, English language fluency was found related to perceived language discrimination but not to perceived racial discrimination (Goto et al., 2002). Nonetheless, the subtleties of perceiving discrimination are still present due to language difficulty. In a study of physicians wherein $28.3 \%$ of the participants have English as a second language, language is deemed a barrier to a successful career and a threat to the patient-physician relationship (Coombs \& King, 2005).

In New Zealand, the New Zealand Immigration Service (NZIS) has set a minimum requirement in English language proficiency for incoming immigrants. For instance, principal applicants for the Skilled Migrant Category (the entry category for most skilled immigrants) should have an overall band score of 6.5 or better and 5.0 or better for the principal and the 
non-principal applicants respectively in the International English Language Testing System (IELTS). If the applicants do not meet these IELTS minimum requirements, they should provide proof of having an English-speaking background (NZIS, 2009).

To test the IPDWS scales' concurrent validity, the mean score of three items of English language proficiency was used. These are the responses to three questions related to writing (Written English Competency), speaking (Spoken English Fluency) and comprehension of the English language (English Comprehension) which gauged the participants' personal assessment of their level of English-language proficiency. The respondents reported their English proficiency from very well to excellent in writing $(M=1.83, S D=.79)$, speaking $(M=1.92 ; S D=.83)$ and comprehension $(M=1.84 ; S D=.79)$. Scores obtained from the three items were calculated as a single composite score for the whole sample.

Table 5.11 shows that majority of the factors have significant positive correlations with English language proficiency with a few exceptions. Hence, for most of the PD factors, higher PD is associated with low levels of English proficiency. The two PD factors that did not correlate significantly with the language proficiency measures were Network ( $\mathrm{r}=-.14$, n.s. $)$ and Derogation $(\mathrm{r}=.14$, n.s. $)$.

\section{Examination of vertical structure - Second order factors.}

A second exploratory factor analysis was conducted to determine the vertical structure or a higher order structure of the factors extracted in the first principal components analysis (PCA). Thus, another PCA was performed using Direct Oblimin with Kaiser 
Table 5.11

Pearson Correlations Among Factors in the IPDWS and English Proficiency

\begin{tabular}{ll} 
& English Proficiency \\
\hline Finding Work: & \\
$\quad$ Non-Local & $.26^{\star \star}$ \\
Non-Recognition of Educ/Exp & $.21^{\star \star}$ \\
Local Requirements & $.25^{\star \star}$ \\
$\quad$ Network & -.14 \\
Work Conditions: & $.20^{\star \star}$ \\
$\quad$ Hindered Career Path \& Unequal Opportunity & $.25^{\star \star}$ \\
$\quad$ Inadequate Compensation & $.29^{\star \star}$ \\
$\quad$ Differing Treatment & $.28^{\star \star}$ \\
Work Relationships: & .14 \\
$\quad$ Exclusion & $.34^{\star \star}$ \\
Derogation & \\
Disharmonious Work Dynamics &
\end{tabular}

Note: $* p<.05, * * p<.01$

Normalization. The means scores of the factors in the first PCA were entered into the analysis. Thus, ten 10 mean scores were used to perform the PCA to establish the higher order structure. Three initial eigenvalues greater than 1 were generated $(3.77,1.42$, and 1.14$)$, but after rotation only two eigenvalues greater than 1 emerged (3.64 and 1.82). Inspection of the scree plot and the pattern matrix also suggested two interpretable factors accounting for $52.10 \%$ of the total variance.

Thus, two factors were extracted. The first higher order factor contains primary factors Non-recognition of Educ and Experience and Network which represent Job-Entry perceptions and the second higher order factor has Hindered Career Path \& Unequal Opportunity, Inadequate Compensation, 
Exclusion, Derogation, Disharmonious Work Dynamics and Differing Treatment which pertains to On-the-Job PD. The primary factor Non-local both loaded in the first and second higher order factors with loadings of .53 and .50 respectively. After computing for the internal consistency coefficients it was decided that the Non-Local factor be retained in the first higher order factor (Job-Entry). Retaining Non-Local in the first higher order factor resulted in a Cronbach's alpha coefficient of .58 , and without it the value drops to .43 . The second higher order factor (On-the-Job) retains a high value of internal consistency with $(\alpha=.82)$ or without $(\alpha=.81)$ the Non-local primary factor. Presented in Table 5.12 are the second order factors or the higher order factors of the IPDWS with the pattern matrix's factor loadings, eigenvalues and their variance and internal consistency coefficients.

Table 5.12

Factor Loadings and Internal Consistency for the Second Order Factors

\begin{tabular}{lcc}
\hline & Factors & \\
Items & $\mathbf{1}$ & $\mathbf{2}$ \\
\hline Factor 1: Job-Entry & .53 & .49 \\
1. Non-local & .55 & \\
2. Non-Recognition of Education and Work Experience & .75 & \\
3. Local Requirements & .65 & \\
4. Network & & \\
& & .73 \\
Factor 2: On-the-Job & & .65 \\
1. Hindered Career Path and Unequal Opportunity & & .79 \\
2. Inadequate Compensation & & .45 \\
3. Exclusion & & .84 \\
4. Derogation & & .74 \\
5. Disharmonious Work Dynamics & 3.77 & 1.44 \\
6. Differing Treatment & 37.73 & 14.37 \\
Eigenvalues & .58 & .82 \\
\% of Variance & .43 & .81 \\
Cronbach Alpha (With Non-Local) & & \\
Cronbach Alpha (With/out Non-Local) & & \\
\hline
\end{tabular}

Note: item loadings belonging to its respective higher order factors are represented in bold face. 


\section{Discussion}

The main objective of this chapter was to come up with a PD measurement that captures immigrants' perceptions of discrimination in New Zealand workplaces as reflected in the themes generated from the focus groups discussions. Since no single scale in the discrimination literature incorporated all of the themes found in the qualitative study, it was deemed necessary to construct a new PD measurement that depicts the PD ideas or concepts reflected in the themes. Hence, the resulting PD measurement (Immigrants' Perception of Discrimination in the Workplace Scales-IPDWS) was a result of an effort to present current discriminatory perceptions of immigrants as reflected in the themes generated from the Focus Groups Discussion. This intention therefore was, foremost, is the examination of the conceptualization or nature or of PD. Then, the creation of new measures came as a resolution to the problem of not being able to find a particular scale that could represents all the concepts of PD found in the themes.

The three scales comprising the IPDWS represent one particular important contribution in understanding how immigrants view their workplace. In addition, IPDWS aimed to capture not only current workplace barriers such as organizational practices and workplace relationships but also barriers that are encountered during the job hunting stage; thereby considering the entire employment cycle of the immigrants' work experience. The scales aim to measure the access and treatment discrimination distinction discussed by Levitin et al., (1971) which could be both under formal and informal structures. The psychometric properties presented in the previous section 
indicated that IPDWS are both reliable and internally valid and henceforth shows promise as a psychometric instrument.

\section{The Exploratory Factor Analyses Outputs}

The initial PCA for the first scale, the IPDWS-Finding Work, generated four factors which is one factor off from the researcher's initial groupings of items. The items on Language/Accent did not come out as a separate factor but instead were integrated in the personal characteristics factor which was eventually termed as being "Non-Local". Hence, having English as a second language is a salient part of being a stranger. There was also another factor that came out as a distinct factor which was obtaining "Local Requirements" wherein migrants have to obtain further education in NZ, get recognition of qualifications obtained overseas from local NZ authorities as well as comply with the local employment practices such as references and accepting jobs to get NZ experience. These items were generated from the FGD discussion and appear to be relevant in the NZ workplace context in general.

The second scale IPDWS-Work Conditions refers to formal structures (Gelfand et al., 2005; Levine \& Leonard, 1984) that hinder the full development and compensation-related entitlements of migrants in the workplace. The initial PCA generated factors that did not simply group the items according to their classification (e.g. promotions/trainings, fringe benefits) but had a deeper meaning in terms of difficulties in career development and coupled with unequal opportunity policies (Hindered Career Path \& Unequal Opportunity). Hence, progression in the organization is deemed possible only if there is an existing perceived fairness of treatment 
towards every employee regardless of their ethnic/cultural background. Another factor "Inadequate Compensation" referred to how the respondents perceive their pay and fringe benefits to be less compared to relevant others within the organization and within similar organizational sectors. The "Differing Treatment" is composed of items that point to policies which differentiate employees in terms of their ethnic background.

The third and the last scale IPDWS-Work Relationships measures perceptions of informal structures which the literature points out to be an important context of discrimination. Initially, the author classified the items according to three levels namely co-workers, supervisors and general/systemic in accordance with the suggestions of the Embedded Intergroup Theory (EIT) by (Thomas \& Alderfer, 1989). However after the initial PCA, the factors that emerged did not support the initial classification as predicted by EIT and the IMCD of Cox (1993). Instead, three factors came up, which were labelled as Exclusion, Derogation and Disharmonious Work Dynamics.

The factor Exclusion contained items pertaining to feelings of being segregated by others due to their ethnic/cultural background. Moreover, Derogation was represented by items related to being the centre of jokes or derogatory comments in the workplace. Lastly, Disharmonious Work Dynamics relates to positive acceptance from the co-workers including supervisors, which was reflected in the recognition of their work contributions and healthy work relationships.

The failure to replicate the levels as originally intended to be captured in this scale (i.e. co-workers, supervisors and general/systemic) may be due to the egalitarian workplace atmosphere experienced by the immigrants in the 
New Zealand workplace in contrast to the patriarchal and hierarchical relationships that characterize countries where migrants came from (mainly from Asia). These perceptions were also stated in the focus groups discussions conducted for the New Zealand immigrant groups. Thus, egalitarian working environments, wherein you can call your boss using his/her first name, is atypical to the former experiences of the predominantly Asian participants. As a result, the derogation factor contained items that are both jokes and negative commentaries which can be conceptually different. More importantly, there was no opportunity to construct more items that could represent blatant or subtle derogation nor exclusion in the workplace because at the onset these items were not intented to represent specifically "derogation" or "exclusion".

Alternatively, the emergence of the derogation and exclusion factors instead of the multi-level perceptions as originally expected may be a reflection of what Smith (2008) would refer to as "the degradation of social existence in the globalizing world" (p.372). He suggests that people are forced into humiliating circumstances (e.g. being stripped down of your respectability and worth) but now react beyond submission and conformity. This means that reactions are not passive responses but are increased awareness and more active response to humiliating circumstances that allow "victims" to be active agents - by either attempting to change the social structures or change themselves (Smith, 2008). Thus, these PD factors could be linked to the changing schema of victims or targets in the age of globalization, as also reflected in humiliation studies, by being more aware of what is happening within their environment and increased willingness to act on them. 
The second exploratory factor analysis through the second PCA generated higher order factors which were Job-Entry and On-the-Job perceptions of discrimination. This was consistent with the proposition of Levitin et al. (1971) on Access and Treatment Discrimination. They described Access Discrimination as unequal treatment that an individual may experience related to the recruitment and selection procedures such as denial of preferred job and lower starting pay. Treatment Discrimination was defined as conditions or differential treatment that limits individuals from realizing his/her full potential in the workplace, like promotions and salary increases (Chung, 2001; Brown \& Ford, 1977).

The first higher order factor Job-Entry is consistent to Access Discrimination while the On-the-Job is consistent with Treatment Discrimination. The second order factors both contain formal and informal forms of discrimination. Levine and Leonard (1984) described formal work discrimination as organizational policies related to hiring, compensation, benefits and the like, while informal work discrimination was described as relational dynamics or interactions and work atmosphere. Based on these results, findings show that Access (i.e., Job-Entry) and Treatment discrimination (i.e., On-the-Job) are not only manifested in the Formal Discrimination category as suggested by Chung (2001) but also in Informal Discrimination (e.g. network perceptions). This finding is a novel framework in the broader understanding of perceptions of discrimination in the workplace as perceived by immigrants. 


\section{The Intercorrelations Output}

At the onset, negatively worded items were reverse scored so that all items had the same score direction. The correlations coefficients of the factors were from low to moderate indicating that the factors were relatively independent from each other. Almost all factors in the IPDWS correlated significantly with each other except for two factors in the Finding Work Scale, Local Requirements and Networking that did not correlate with the other PD factors.

Local Requirements correlated significantly with all other factors except for the ones in the Work Relationship Scale. It means that satisfying local requirements is not related to PD on workplace interactions. Further, Networking was significantly correlated with other factors except for Hindered Career Path/Unequal Opportunity (Work Conditions Scale) and the three factors in the Work Relationships Scale. Both factors (Local Requirement \& Networking) did not relate well with informal structures (work interactions/relationships). Items of the two factors are associated more with formal structures like hiring policies (qualifications, work experience, referrals) and compensation issues (salary, benefits, trainings). Networking was also not significantly related to Non-Recognition of Educ and Experience. Thus these two concepts are not related for the participant sample which makes sense since having your own qualifications and experience from overseas may not affect how well you expand your network in the current host country. One's skill to network may be more attributable to personality and how well you communicate with other people.

Finally, correlations differed significantly for Networking and Differing Treatment, showing a negative relationship. It illustrates that the 
higher the need to network in order to increase chances of being hired, the less it was perceived that organizations apply different policies to different target groups. Hence, the importance of knowing people in the organizations diminishes the perception of unfair policies in organizations. The correlation between Networking and Inadequate Compensation was also negative but it did not reach significant levels.

\section{Limitations of the Study}

The strengths of this study are moderated by some inherent weaknesses. First, the psychometric properties of IPDWS have been evaluated with 155 immigrant participants with a high proportion of Asian (specifically Filipino) participants who were mostly working as professionals. Hence, there is a need to look into a wider representative sample with more diverse ethnic, job, and age backgrounds to reassess the factor structure of the scales. It remains to be seen if the factor structure will hold across immigrant groups in less educated, lower position levels and more diverse age groups. Thus, the strength of IPDWS can be validated by future studies that will administer the scales to various groups. The next chapters will attempt to address this concern.

Secondly, if the primary factors are considered in the IPDWS, there are factors which only have 3 items (Networking and Differing Treatment) which would be unacceptable if the retention criteria suggested by Guadagnoli and Velicer (1988) and Velicer and Fava (1998) were followed. According to these authors, factors with small number of items (3 or less) are acceptable when the sample size is large (>300) and the items loadings are high (.80). Our sample size is considered to be a bit above their quoted "small" sample size which is 
$<150$ and the factor loadings were above the minimum recommended loading of 0.40 .

Hence, although the factor loading of Networking and Differing Treatment had a minimum value of 0.53 , and the On-the-Job higher factor had a minimum item loading of .45 , some of the factors have less than that of the suggested Cronbach' alpha coefficient of 0.80 . Even with the results of the PCA done to determine higher order factors, the internal consistency coefficients of one factor was also below .80. Thus, analysis should be undertaken with caution until a confirmatory factor analysis has been performed on the scales and until the scales are administered to a bigger and more diverse sample.

Lastly, the IPWDS were designed based on the perceptions of discrimination of migrants within the New Zealand workplace context. Although some of the items in the scale were taken from established general workplace discrimination scales from abroad, other items originate from the focus groups discussions that were conducted in New Zealand. Therefore, the scale may not be applicable outright to participants working and living external to New Zealand unless some modifications are made on the scales.

\section{Suggestion for Future Study}

The sample was also limited to immigrant employees hence the applicability of the IPDWS to other groups need to be further investigated. It remains to be seen if the factor structures found in the current study findings will hold true for other samples. 


\section{Summary and Conclusions}

A review and evaluation of existing measurements used in studies related to perceptions of discrimination as a function of race or ethnicity was conducted to determine existing PD measurements/scales that may be relevant to the current area of study. Since there was no single existing scale that satisfied the objectives set for examining immigrants' PD in the workplace, steps were taken to construct a quantitative measure that will be utilized to capture a larger sample of participants to generate a more randomized, generalizable and statistically reliable data.

The findings provided evidence that PD has multiple dimensions. I also tried to address issues of content, reliability and validity issues on the pilot version of the IPDWS. The statistical analysis eventually resulted in a 2higher order factors with labels Job-Entry and On-the-Job Perceptions of Discrimination. Based on existing guidelines on exploratory factor analysis, the structure of the scale passed existing criteria on reliability and validity. The results demonstrated that factor structures of IPDWS have good psychometric properties and can thereby be used in the studies described in the succeeding chapters of this thesis. 


\section{Chapter 6 \\ OUTCOMES OF PERCEIVED DISCRIMINATION: \\ ORGANIZATIONAL AND PSYCHOLOGICAL WELL-BEING (STUDY 3-B)}

\section{Introduction}

This chapter presents the "downstream" analysis (Schnittker \& McLeod, 2005) of the perceived discrimination (PD) process by looking into two groups of probable outcomes - organizational and psychological wellbeing. To recall, one major objective of this thesis is to investigate the incremental effects of PD on various outcomes over and above other demographic variables, as illustrated in the right-hard portion of Figure 3.1 in Chapter 3.

Ensher et al. (2001) pointed out the importance of linking employee perceptions and outcomes. They believed that looking into the perceptions of employees is important since differing opinions hinder the creation of an integrated organizational culture (shared values and beliefs) that have a strong influence on organizational policies. Thus some employees may perceive policies as appropriate and some would not. All in all, PD may also influence individuals' attitudes and behaviour that could in turn affect their organizations (Gutek et al., 1996; Kanter, 1977; Weick, 1995). These attitudes and behaviours could subsequently either improve or harm the organization's performance outcomes. More importantly, examining employee PD allows us to predict when perceptions are likely to develop and in what context of employment relations these perceptions are likely to occur (Balser, 2000). 
Although general employee perceptions and their impact on human resource issues has been explored in previous studies, little focus has been accorded to the reactions of the individuals to discriminatory situations and the resulting effects on organizational and job outcomes (Ensher et al., 2001; Gutek et al., 1996). Thus, perceptions of whether an individual's opportunities are equal to the opportunities of other employees in the organization have an effect on the individual's attitudes and behaviour towards the organization. However, little research has addressed this issue (Gutek et al., 1996). The minimal focus on employee perceptions renders the search for knowledge on workplace inequality as incomplete (Balser, 2000). Thus, a call for more research on perceptions has been issued by Dipboye and Collela (2005).

Moreover, the issue of increasing globalization and the need to address competition for skilled human resource has been mentioned in previous chapters. Thus, the need to look into the welfare of a new generation of skilled workers searching for economic opportunities outside their home country is an increasing concern. Skilled immigrants in general face an array of challenges once they step into a foreign country. Immigrants are often facing economic, social and psychological barriers in the workplace more than the economic advantages that they hoped to gain in moving to a new place. After the initial experience of culture shock (Ward et al., 2001), other problems confronting immigrants that affect their settlement outcomes include experiencing discrimination (IOM, 2005; Ward \& Leong, 2006), being exploited as cheap labour (Marsella \& Ring, 2003), being underemployed (Slack \& Jensen, 2002; Ward \& Masgoret, 2007), experiencing acculturative stress (Berry, 2006) and social exclusion (IOM, 2005). As a whole, Marsella and Ring (2003) describe 
the experience of migration as dangerous to the "life, limb and psyche" (p.9) of immigrants.

There is a gap in discrimination research on immigrants' discrimination perceptions and its effects on outcomes in the workplace. The acculturation literature has provided some angles on immigrants' viewpoints in relation to unfair treatment. For instance, a review on the effects of prejudice and discrimination by Ward et al. (2001) showed that perceived racism and/or $\mathrm{PD}$ could have detrimental effects on immigrants such as negative consequences on psychological well-being, adaptation, distress, and drug-related problems among others.

Thus, this study is a major contribution to the literature since it addresses several research gaps in organizational psychology research, namely, a) it contributes to the growing number of empirical studies on target's perspectives; b) it addresses the gap in organizational research in terms of increasing the number of studies linking employee PD and organizational outcomes; c) it provides a novel approach by additionally looking at the relationship of PD and psychological well-being outcomes in addition to organizational outcomes; and d) it focuses on immigrants who may be experiencing multiple discrimination in the workplace due to their immigrant status on top of their demographic characteristics such as ethnicity, gender, language proficiency and age.

\section{Perceived Discrimination and Organizational Outcomes}

This study will be looking into three organizational outcomes namely job satisfaction, organizational commitment and turnover intentions. An important gauge of an organization's future success is employee attitudes 
(Hurst, 1995). Attitudes are results of affective reactions to past experiences (Eagly \& Chaiken, 1993). As such, attitudes like satisfaction and commitment in organizational settings are deemed important since these variables could predict if employees will remain affectively connected to an organization or would quit (Holton \& Russel, 1999; Meyer \& Allen, 1997). These two variables were also considered to be important aspects of employee attitudes that are likely to be influenced by PD (Ensher et al., 2001). Moreover, there is a need to reiterate that loss of talented individuals for reasons of voluntary resignations can have serious effects on organizational performance (Balser, 2000). Thus, this study deemed it also important to look into turnover intentions, another attitudinal component that could lead to a behavioural manifestation (actual turnover), as another outcome of an employee's cognitive experience.

\section{Job Satisfaction}

Job satisfaction is defined as a pleasurable or positive emotional state resulting from the appraisal of one's job or job experiences (Locke, 1976). It is one of the most widely researched job outcomes in organizational psychology although results remain contradictory and vary across social policies, economic context or countries (Judge, Parker, Colbert, Heller, \& Elies, 2001). Job Satisfaction has been found by cross-cultural researchers to have two facets: the intrinsic and extrinsic facets. The extrinsic facet pertains to pay and promotion while the intrinsic facet pertains to the job itself, as well as supervisors and co-workers (Judge et al., 2001). In various studies, job satisfaction has been related to organizational variables like organizational commitment either as predictor or as a contributor (Johnson \& Jones-Johnson, 
1992; McNeese-Smith \& Nazarey, 2001; Sikorska-Simmons, 2005). Additionally, job satisfaction has been reported to be negatively related to organizational commitment (Ragins \& Cornwell, 2001) and turnover intentions (Mowday et al., 1982) in gay and lesbian studies.

Although there is an extensive literature on job satisfaction, a review by Jayaratne (1993) found that there is a lack of research on job satisfaction relating to race in general and differences in PD in particular. This scarcity of specific studies may contribute to the lack of agreement among scholars on the issue of race, perceived discrimination and job satisfaction (Cox \& Nkomo, 1993; Jayarante, 1993). An example of a study that directly linked race with job satisfaction is that of Mueller, Finley, Iverson, and Price (1999). They concluded that racial composition affects job satisfaction such that White teachers in Black-dominated schools have less job satisfaction. However, non significant findings were reported for Black Teachers in White-dominated schools.

Nonetheless, the minimal studies included in the meta-analysis in Chapter 2 showed that PD has a negative relationship with job satisfaction and this is the strongest effect found among the other correlates that were included in the analysis. Specifically, Sanchez and Brock's (1996) study on Hispanic employees from various US organizations showed that PD was negatively correlated with job satisfaction. Similarly, Ensher and colleagues (2001) illustrated the different levels of analysis in their study of 366 ethnically diverse US employee sample by focusing on job-related outcomes of perceived discrimination in terms of co-worker, supervisor and organizational discrimination. They found that job satisfaction was predicted by 
organizational-level discrimination and supervisor-level discrimination. Lastly, Deitch et al. (2003) also showed that job satisfaction is negatively associated with PD in their study, which had a mixed sample of Black and White employees.

\section{Organizational Commitment}

The second dependent variable is organizational commitment, which is the psychological attachment of the individual to his/her organization (Mowday, Porter \& Steers, 1982, O’Reilly \& Chatman, 1986). Meyer and Allen (1991) further categorized organizational commitment into the following three components: affective commitment (the desire component), continuance commitment (the need component) and normative commitment (the obligation component). Affective commitment is defined as employee's emotional attachment to, identification with, and involvement in the organization (Mowday et al., 1982). Continuance commitment is the employee's awareness of the costs associated with leaving the organization (Becker, 1960, in Yao \& Wang, 2006). Normative commitment, on the other hand, is the employee's feeling of obligation to continue working in an organization (Meyer \& Allen, 1991).

Organizational commitment has been found to be related to various employee attitudes and behaviours including job satisfaction, extra-role behaviour, goal and value congruence, behavioural investment in the organization, and low turnover (Hackett, Bycio, \& Hausdorf, 1991; Moorman, Niehoff, \& Organ, 1993; Mowday et al., 1982; Wasti, 2003; see also metaanalysis by Meyer et al., 2002). All three dimensions of organizational commitment have been found to positively correlate with job satisfaction (e.g. 
Mathieu \& Zajac, 1990) and negatively with turnover intentions and actual turnover (Chen \& Francesco, 2000; Konovsky \& Cropanzano, 1991; Meyer, Allen, \& Smith, 1993; see meta-analysis of Fischer \& Mansell, 2005).

Among the three dimensions, affective commitment has been found to be the best predictor of attitudes and behaviours in organizations including turnover intentions (Feather \& Rauter, 2004; Fischer \& Mansell, 2005, Wong, Ngo \& Wong, 2002). Cultural differences were also found in the relationship of affective commitment and turnover intentions. Specifically, Fischer \& Mansell (2005) found that affective commitment's negative relationship with turnover intention is stronger in individualistic cultures. Moreover, they also found that greater individualism was also related to the less negative associations between normative commitment and turnover intentions.

Commitment to organizations has been considered as most stable across time compared to other organizational variables such as job satisfaction (Porter, Steers, Mowday \& Boulian; 1974; Yao \& Wang, 2006). Hence, looking at covariates of organizational commitment, either as antecedents, correlates and outcomes has garnered increasing interest for over a decade (see Meyer \& Allen, 1997).

Maintaining levels of employee commitment, specifically for minorities and women, is a current major concern for organizations (Ensher et al., 2001). Studies looking at ethno-racial demographic differences, Whites have been reported to have lower organizational commitment than Blacks (Tsui et al., 1992; Lankau \& Scandura, 1996). In contrast, White teachers in White-dominated schools have been found to have higher career commitment than Black teachers in White-dominated schools (Mueller et al., 1999). 
In relation to perceptions, workplace discrimination perceived by minorities can lower organizational commitment, eventually resulting to changing means of occupation (Ragins \& Cornwell, 2001; Shallenbarger, 1993). The minimal studies done on race/ethnicity related PD have also shown the negative impact of PD on organizational commitment. Specifically, organizational commitment has been found to be negatively related to PD among Hispanic employees from various United States organizations (Sanchez \& Brock, 1996). On a multi-level perception approach by Ensher et al.'s (2001), multi-level perception approach found that organizational-level discrimination and supervisor-level discrimination predicted organizational commitment. Unfortunately, the investigation of the three dimensions of commitment in relation to PD is yet to be achieved. Hence, this will be addressed in this Chapter.

\section{Turnover Intention}

The third job outcome is turn-over intention which is defined as the intention to eventually leave the job and/or organizations (Bluedorn, 1982; Low, Cravens, Grant, \& Moncrief (2001). Turnover intention reflects the probability that an individual will leave his/her job within a certain period (Sousa-Posa \& Henneberge, 2004). Thus, it is considered a part of the employee's attitude towards the organization. Similar to job satisfaction, intention to quit has been studied extensively in management literature (e.g., Foley et al., 2005; Randal, Cropanzano, Bormann, \& Birjulin, 1999) and is one of the most common dependent variable used in studies (e.g., Chang, 1999; Wong et al., 2002; Yao \& Wong, 2006). Turnover intent may or may not lead to actual employee turnover (Chen, Hui \& Sego, 1998) but it has been 
found to be the strongest predictor of actual employee turnover (Rosin \& Korabik, 1991; Stroh, Brett, \& Reilly, 1996).

Employee intentions to stay or leave an organization have been regarded as less important than determining levels of performance in the job (Mowday et al., 1982). However, employee resignations can have adverse and costly repercussions for business operations and performance (Balser, 2002; Meyer, Paunonen, Gellatly, Goffin, \& Jackson, 1989). Moreover, intentions to leave, whether it will or it will not lead to actual turnover has been found to be related to negative job attitudes and less job satisfaction, both of which are important indicators of good performance (Mowday et al., 1982).

In relation to race/ethnicity related discrimination, studies have found inconsistent links between turnover intentions and perceived discrimination. More than a decade of research has shown that White faculty employees have higher intentions to stay in the workplace than Black faculty employees (Davis, 1985), which also coincides with greater faculty turn-over among Blacks faculty members than Whites. In contrast, Tsui et al (1992) found that Blacks have lower turnover intentions than Whites. A direct positive relationship was found between perceived discrimination and turnover intentions in studies by Foley et al. (2005) and Shaffer et al. (2000). Perceptions of discrimination can also cause women and minorities' high turnover and their propensity to set up their own businesses (Dickerson, 1998; Federal Glass Ceiling Commission, 1995).

Overall, job satisfaction, organizational commitment and turnover intentions have been found to be associated with perceived discrimination in a few studies. Most of the findings linking organizational outcomes with 
race/ethnicity related perceptions come from studies that directly associate demographic information with job outcomes. Ample attention has been accorded to these outcome variables in gender discrimination studies, diversity research or studies using Relational Demography Theory (Tsui et al., 1992) as a framework thus using demographic information such as gender and ethnicity (see review of Riordan et al., 2005). Therefore, discrimination was implied based on the disadvantage of minorities, with a few studies investigating job outcomes as a direct effect of perceived discrimination in organizations (e.g. Ensher, 2000; Sanchez \& Brook, 1996). More studies are needed to establish the causality of the relationship between PD and job outcomes, and to distinguish between the different components of commitment. More importantly, few of these studies have focused on immigrants. Nonetheless, the existing studies on organizational outcomes provide a consistent pattern that could be inferred in relation to perceived discrimination. Thus, the following hypotheses are proposed:

Hypothesis 6.1: The more the immigrants perceive discrimination, the lower their job satisfaction.

Hypothesis 6.2a: The more the immigrants' perceive discrimination, the lower their affective commitment.

Hypothesis 6.2b: The more the immigrants' perceive discrimination, the lower their continuance commitment.

Hypothesis 6.2c: The more the immigrants' perceive discrimination, the lower their normative commitment.

Hypothesis 6.3: The more the immigrants' perceive discrimination, the higher their intent to leave. 


\section{Perceptions of Discrimination and Psychological Well-being}

Most studies on psychological well-being have been centered on acculturation, gender studies or the youth population. Various studies have found perceived discrimination to predict different aspects of immigrants' psychological well-being or health, such as depression (Finch et al., 2000; Noh, Beiser, Kaspar, Hou \& Rummens, 1999; Pernice \& Brook, 1996), distress and anxiety (Kessler, Mickelson, \& Williams,1999; Pernice \& Brook, 1996); life satisfaction (Brown, 2001; Liebkind \& Jasinskaja-Lahti, 2000b; Ying, 1996); behavioral problems (Liebkind \& Jasinskaja-Lahti, 2000b; Verkuyten, 1998); and physical health (Finch et al., 2001; Krieger \& Sidney, 1996; see review of Harrell, Hall, \& Taliaferro, 2003). The impact of PD on physical health is more indirect and slower while it is more direct, immediate and strong for psychological health (Gallo \& Matthews, 1999; Williams, Neighbors, \& Jackson, 2003). Furthermore, the negative consequences of perceived discrimination on immigrants' well-being is viewed to be long lasting because discrimination experiences are often chronic and reflect a stable perception of a discriminatory and prejudiced social context (Allison, 1998; Swim, Cohen, \& Hyers, 1998).

Although the literature is rich in studies on the impact of perceived discrimination on immigrants' psychological well-being (see Chapter 2), the workplace context has not been given ample attention. This study intends to address this concern by considering the two psychological well-being variables that represent both the negative and positive outlooks in life in the workplace context using immigrant samples. These two variables could 
provide a holistic representation of an immigrant's overall well-being in the workplace by looking beyond their job outcomes.

Two additional outcome variables were considered in the analysis to determine the effects of PD psychological well-being of the immigrant sample. These were 1) psychological distress which is a negative assessment of the individual's mental health status, using the General Health Questionnaire (GHQ-12), a self-rated health outcome specifically measuring psychological well-being (Goldberg, 1972), and 2) life satisfaction described as the extent to which a person feels positively or negatively towards his/her current life in general (Diener, 1984). Life satisfaction, the cognitivejudgemental aspect, was identified by Diener (1984) as one of the three independent components of subjective well-being, the other two are the positive and negative affects (emotional states).

In the past, social scientists have shown more interest on psychological distress rather on life satisfaction (Arrindell, Heesink \& Feij, 1999) as evidenced by higher number of articles on psychological distress listed in the Psychological Abstracts (Myers \& Diener, 1997). This could be attributed to the inherent concern of Psychology towards human misery and pain rather than predictors of positive affectivity (de Rivera et al., 1989; Diener, 1984; Ryff, 1989). Growing interest has been accorded towards life satisfaction research in the past three decades (e.g. Diener, 1984, 1994; Costa \& McCrae, 1980, McRae \& Costa, 1995, Myers \& Diener, 1995). Generally, findings show that life satisfaction is negatively related to psychological distress (Arrindell et al., 1999; Arrindell, Meeuwesen, \& Huyse, 1991; Gouveia, 
Milfont, da Fonseca, \& de Miranda Coelho, 2009) although no causal relationship has been established (Gouveia et al., 2009).

The psychological well-being of minority groups has been given main attention by researchers in relation to various antecedents such as sociodemographic factors (e.g. race, ethnicity, gender, etc), prejudice and discrimination (Mays et al., 1996). In the US, perceptions of race-based discrimination by Black women was found to be a significant predictor of job stress specifically for the young adult and educated (Mays et al., 1996). Racebased PD by African American adolescents was also related to depressive symptoms (Brody et al., 2006), to event-specific and global psychological distress (Sellers \& Shelton, 2006) and higher depression and perceived stress but not to psychological well-being (Sellers et al., 2006). For Latinos in the US, higher PD was found related to higher psychological distress (Moradi and Risco, 2006). Likewise, a study involving Asian Americans of Indian ancestry found that PD negatively effects psychological well-being and is positively related to distress (Lee, 2003).

European-based studies on immigrants also found that higher PD results in higher psychological stress symptoms like depression, anxiety and psychosomatic stress (Jasinkaja-Lahti \& Liebkind 2001); predicts depression, anxiety and psychosomatic stress (Jasinskaja-Lahti et al., 2007); and is associated with distress symptoms (Oppedal et al., 2004). Furthermore, the ICSEY study (Berry et al., 2006) conducted on youth immigrants in thirteen countries showed strong negative relationships between PD and psychological adaptation (e.g. life satisfaction) and socio-cultural adaptation (e.g. school adjustment and behavior problems). 
Lastly, in the Oceana Region, Pernice and Brook (1996) found that depression and higher anxiety levels were associated with the experience of discrimination by immigrants who have settled in New Zealand for at least 5 years. Similarly, Australian immigrants' PD and dissatisfaction were related to low mental health scores (McDonald et al., 1996). Relatively low scores were similarly reported for Australians in the national mental health survey data used by Donath (2001). In New Zealand, Pernice, Trlin, Henderson and North (2000) did not find any difference in mental health scores for the three NZ immigrant groups coming from China, India and South Africa (107 participants) divided into the unemployed and employed categories. All groups reported low psychological health scores. They attributed the low scores of the employed participants to work stress and being employed in jobs that are below their educational qualifications and experience. Thus, the findings of the aforementioned studies generally suggest the following hypotheses:

Hypothesis 6.4: The more the immigrants' PD, the higher the levels of their psychological distress.

Hypothesis 6.5: The more the immigrants' PD, the less satisfied they are with their lives.

To recap, this study (Study 3-B) has two major specific objectives. First, it examines PD among immigrants. This will contribute to the paucity of research on immigrants' perceptions not only in New Zealand but also in the international literature. Secondly, the study intends to determine if immigrants' $\mathrm{PD}$ are related to job and psychological well-being outcomes. Organizational outcomes that are of interest in this study are job satisfaction, 
organizational commitment and turnover intentions. The study intends to capture attitudinal (job satisfaction, organizational commitment and turnover intentions) dimensions of organizational outcomes. Furthermore, the study also aims to assess the impact of PD on psychological well-being variables by examining two dimensions. These well-being variables are psychological stress (considered as negative dimension) and life satisfaction (regarded as positive dimension). This particular approach has not been done in New Zealand and to the best of my knowledge, the focus (immigrants in the workplace) and the outcome variables have not yet been examined in one single study in the international literature. Thus, this study attempts to contribute a distinct and a novel input to the literature.

\section{Method}

\section{Participants}

The same set of participants described in Chapter 5 will be used for this analysis. The responses on the questionnaire which corresponds to the results of the exploratory factor analysis of the IPDWS-Revised version are included for the analysis.

\section{Measures}

For measuring PD, the PD scales constructed in Chapter 5 (Study 3-A) were used to assess the independent variable. Moreover, the dependent variables were assessed using validated measurements gathered from the literature. All have exhibited good reliability coefficients in previous studies that have used them. Similarly, this study sample also demonstrates average to 
strong reliability coefficients from these existing scales as listed in Table 6.1. Thus, the questionnaire contained the following scales.

\section{Independent variable.}

Immigrants' Perceptions of Discrimination in the Workplace Scale Revised Version (IPDWS-R). The revised versions of the IPDWS were used to measure PD. Factor structures and the reliabilities were assessed in the previous chapter. Please see Chapter 5 for the detailed description of the scales.

\section{Dependent variables.}

Job Satisfaction was assessed through a 9-item scale asking respondents on the extent to which he/she feels positively or negatively toward his/her work. Items came from the original items of the scale used by Podsiadlowski (2002) and Ensher et al. (2001) with secondary related sources including Neuberger and Allerberck (1978, in Podsiadlowski, 2002), and Dockery and Muchinsky (1977, in Podsiadlowski, 2002). Responses were on a 7-point scale ( $1=$ Not at all satisfied to $7=$ Very much satisfied). Higher responses reflect more satisfaction in their job. For example, one item was "How satisfied are you with your working conditions?" The reliability coefficient for this study's sample is $\alpha=.90$.

Organizational Commitment was measured using 15 items of the threecomponent model (affective, continuance and normative) of commitment developed by Meyer and Allen (1991). Each component was measured by 5 items on a 7-point item scale ( $1=$ strongly disagree and $7=$ strongly agree). Sample items for the components are: a) affective commitment - "I would be very happy to spend the reset of my career with this organization"; b) 
continuance commitment - "It would be too costly for me to leave my organization in the near future"; and c) normative commitment - "I feel a sense of moral obligation to remain in this organization". Reliability coefficients in this study were good, with a Cronbach's $\alpha$ of $.87, .82$, and .76 for affective, continuance and normative dimensions respectively.

Turnover intentions were measured using a single question ("how would you rate your chances of quitting this job") and specifying four time intervals (e.g. in the next three months) reflecting the respondents' intent of quitting their job. This scale was based on the original items of Bluedorn (1982) and Low et al. (2001), with responses on a 7-point ( $1=$ very low to $7=$ very high) scale. Higher scores reflect higher intentions to quit the job. The Cronbach's alpha for this study is $\alpha=.88$.

Psychological Distress was measured through the 12-item General Health Questionnaire (GHQ-12) wherein respondents were asked to report on the state of their psychological well-being. GHQ was developed by Goldberg (1972) and it is a widely used scale for tapping minor psychiatric disturbances in community and applied settings. This 12-item scale is just one of the 5 standard versions having different number of items. A sample item from the scale includes "Been losing confidence in yourself?" Participants were asked to respond to a 4-point scale (e.g. $1=$ not at all, $4=$ much more than usual) with higher scores signify greater disturbance. Ratings of the items were added to compute a global distress score ranging from 0 to 36 , with higher scores indicating higher levels of psychological distress. The Cronbach alpha for this study is $\alpha=.89$. 
Life Satisfaction was measured through the Satisfaction With Life Scale (SWLS) by Diener, Emmons, Larsen, and Griffin's (1985). This scale asks about satisfaction in the conditions of life in general. It is a 5-item scale (e.g. "The conditions of my life are excellent"). Responses ranged from 1 (Strongly disagree) to 7 (Strongly Agree) with higher scores reflecting more satisfaction in life. The scale has been used in previous adaptation studies with various youth ethnic groups in 13 different countries (Berry, Phinney, Sam \& Vedder, 2006), migrant groups in New Zealand (Pernice, et al., 2000) and international students in New Zealand (Ward, Fischer, Lam \& Hall, 2009). The scale has consistently shown good alpha coefficients and validity. The reliability coefficient for this study is $\alpha=.82$.

\section{Control variables.}

Participants were asked to respond to various questions pertaining to some personal and job-related information. The questionnaire also included a self-rated English language proficiency scale containing three questions related to writing (Written English Competency), speaking (Spoken English Fluency) and comprehension of the English language (English Comprehension) intended to measure the participants' personal assessment of their level of English-language proficiency on a 5-point scale, ratings of 1 (excellent) to 5 (can’t write/speak/understand English). Scores obtained on the three items were calculated as a single composite score for the immigrant sample $(\alpha=.55)$.

The demographic information used as control variables in this study were age, gender, income and English language proficiency. Previous studies have shown support on the confounding effects of these variables on the 
outcomes currently being tested (Barry \& Grilo, 2003; Gibbon et al., 2004; Kirchmayer, 1995; 2002; Pavalko, 2003; Romero \& Roberts, 1998).

\section{Procedure}

This study forms part of the survey conducted for the pilot testing of the IPDWS-Pilot version (see the previous chapter for the procedures used).

\section{Results}

\section{Descriptives}

The psychometric properties of all the variables used in this study are reported in Table 6.1. As shown in the table, the variables demonstrated acceptable to strong reliabilities having alpha coefficients ranging from .61 to .90. The mean scores for the subscales in the Job-entry category ranged from $2.48(S D=.86)$ to $3.56(S D=.97)$ showing average to moderately high perceptions of discrimination during the job hunting stage. Perceptions of discrimination subscales in the On-the-Job category, on the other hand, have mean scores ranging from $1.76(S D=.89)$ and $2.95(S D=.55)$ reflecting low to moderate perceptions related to practices and interactions in their current jobs or organizations. Thus, the participants' perceptions of pre-employment discrimination were slightly higher than their perceptions of discrimination in their current work.

Job satisfaction had a mean score of $4.97(S D=1.06)$ indicating a moderately high level of satisfaction of participants in their jobs. Further, the mean scores for affective, continuance and normative dimensions of Organizational Commitment are $M=4.39(S D=1.32), M=4.09(S D=1.32)$, and $M=3.85(S D=1.17)$ respectively. These figures reflect that respondents' 
Table 6.1

Descriptive Statistics and Reliability Coefficients

of Predictor and Outcome Variables

\begin{tabular}{|c|c|c|c|c|}
\hline & $\begin{array}{c}\text { Range of } \\
\text { Responses }\end{array}$ & $M$ & $S D$ & $\begin{array}{l}\text { Cronbach's } \\
\text { Alpha }\end{array}$ \\
\hline \multicolumn{5}{|l|}{ PD Factors } \\
\hline \multicolumn{5}{|l|}{ Job-Entry: } \\
\hline Non-Local & $1-5$ & 2.48 & 0.86 & .88 \\
\hline $\begin{array}{l}\text { Non-Recognition of Education \& } \\
\text { Experience }\end{array}$ & $1-5$ & 2.66 & 1.13 & .86 \\
\hline Local Requirement & $1-5$ & 3.12 & 0.99 & .66 \\
\hline Network & $1-5$ & 3.56 & 0.97 & .67 \\
\hline \multicolumn{5}{|l|}{ On-the-Job: } \\
\hline $\begin{array}{l}\text { Hindered Career Path \& Unequal } \\
\text { Opportunity }\end{array}$ & $1-5$ & 2.40 & 0.79 & .90 \\
\hline Inadequate Compensation & $1-5$ & 2.59 & 0.81 & .81 \\
\hline Differing Treatment & $1-5$ & 1.76 & 0.89 & .61 \\
\hline Disharmonious Work Dynamics & $1-5$ & 1.82 & 0.79 & .89 \\
\hline Exclusion & $1-5$ & 1.91 & 0.87 & .87 \\
\hline Derogation & $1-5$ & 2.95 & 0.55 & .89 \\
\hline \multicolumn{5}{|l|}{ Outcomes: } \\
\hline Job Satisfaction & $1-7$ & 4.97 & 1.06 & .90 \\
\hline Affective Commitment & $1-7$ & 4.39 & 1.32 & .87 \\
\hline Continuance Commitment & $1-7$ & 4.09 & 1.32 & .82 \\
\hline Normative Commitment & $1-7$ & 3.85 & 1.17 & .76 \\
\hline Turnover Intentions & $1-7$ & 3.43 & 1.89 & .88 \\
\hline Psychological Distress (GHQ-12) & $0-36$ & 10.90 & 6.29 & .89 \\
\hline Life Satisfaction (SWLS) & $1-7$ & 4.74 & 1.05 & .82 \\
\hline
\end{tabular}

emotional attachment to their organizations, the perceived cost of leaving their organization, and being obligated to their organizations, were slightly above average. Turnover Intentions has a mean score of $3.43(S D=1.89)$ showing that participants had a moderate intent to leave their jobs in their future. The Psychological Distress measure resulted in a mean score of $10.90(S D=6.29)$ which is significantly higher $t(150)=1.92, \mathrm{p}<.001$ than the mean score for Australian employees (see in Donath, 1991). This illustrates that the immigrant sample in this study were displaying greater levels of mental stress than the Australian norm sample. However, the mean mental health score of 
the participants in this study is significantly lower than the mental health scores of NZ immigrant samples in Pernice et al.'s (2000) study involving Chinese $(N=36)$, South African $(N=35)$ and Indian samples $(N=36)$. Finally, the mean score for Life Satisfaction is $4.74(S D=1.05)$ which means that participants' satisfaction in life are above average. No norm for immigrant working samples could be used as comparison for Life Satisfaction.

\section{Intercorrelations}

Significant correlations among the predictor and criterion variables (see Table 6.2) ranged from .16 to .68 thereby eliminating the problem of multicollinearity. Moreover, the correlations between predictor and outcome variables were mostly in the expected direction except for $P D$ and Continuance Commitment (correlations showed positive relationship instead of the expected negative correlation).

Job satisfaction had significant correlations with the eight PD factors except for Local Requirements and Network. Thus, among the organizational outcome variables, job satisfaction is related to almost all of the PD factors. Affective Commitment correlated significantly with six PD factors except for Non-local, Local Requirements and Derogation subscales. Continuance commitment was significantly related to seven PD factors except for Network, Adequate Compensation and Differing Treatment. Notably, only Continuance commitment showed positive significant correlations with PD factors which are contrary to the expected negative direction between PD and organizational commitment dimensions. Moreover, among the organizational commitment dimensions, only Normative Commitment was not related to any PD factors, 
Table 6.2

Correlation Matrix for the Immigrants' Perceptions of Discrimination in the Workplace Scale and Dependent Variables

\begin{tabular}{|c|c|c|c|c|c|c|c|}
\hline Factors/Variables & $\begin{array}{c}\text { Job } \\
\text { Satisfaction }\end{array}$ & $\begin{array}{l}\text { Affective } \\
\text { Commitment }\end{array}$ & $\begin{array}{l}\text { Continuance } \\
\text { Commitment }\end{array}$ & $\begin{array}{l}\text { Normative } \\
\text { Commitment }\end{array}$ & $\begin{array}{l}\text { Turnover } \\
\text { Intentions } \\
\end{array}$ & $\begin{array}{c}\text { Psychological } \\
\text { Distress }\end{array}$ & $\begin{array}{c}\text { Life } \\
\text { Satisfaction }\end{array}$ \\
\hline 1. Non-Local & $-.33^{\star \star \star}$ & -.09 & $.23^{\star *}$ & .08 & .14 & $.27^{\star \star}$ & $-.34^{\star \star \star}$ \\
\hline 2. Non- Recog of Educ \& Exp & $-.21^{\star}$ & $-.17^{\star}$ & $.29^{\star \star \star}$ & -.02 & .06 & $.17^{\star}$ & $-.23^{\star \star}$ \\
\hline 3. Local Requirements & -.15 & .01 & $.18^{\star}$ & .07 & .07 & .12 & $-.16^{\star}$ \\
\hline 4. Network & .03 & .14 & .05 & .00 & .00 & .07 & -.01 \\
\hline $\begin{array}{l}\text { 5. Hindered Career Path \& Unequal } \\
\text { Treatment }\end{array}$ & $-.68^{\star \star \star}$ & $-.36^{\star \star \star}$ & $.26^{\star \star}$ & -.04 & $.33^{\star \star \star}$ & $.41^{\star \star \star}$ & $-.42^{\star \star \star}$ \\
\hline 6. Indequate Compensation & $-.59^{\star \star \star}$ & $-.25^{\star \star}$ & .09 & .09 & $.30^{\star \star \star *}$ & $.32^{\star \star \star}$ & $-.35^{\star \star \star}$ \\
\hline 7. Differ Treatment & $-.38^{\star \star \star}$ & $-.17^{\star}$ & .04 & .04 & $.18^{\star}$ & .11 & $-.18^{\star}$ \\
\hline 8. Exclusion & $-.50^{\star \star \star}$ & $-.38^{\star \star \star}$ & $.19^{\star}$ & .06 & $.29^{\star \star \star}$ & $.30^{\star \star \star}$ & $-.33^{\star \star \star}$ \\
\hline 9. Derogation & $-.24^{\star \star}$ & -.10 & $.16^{\star}$ & -.10 & .14 & $.25^{\star \star}$ & $-.30^{\star \star \star *}$ \\
\hline 10. Disharmonious Work Dynamics & $-.52^{\star \star \star}$ & $-.27^{\star \star}$ & $.16^{*}$ & .15 & $.23^{\star \star}$ & $.33^{\star \star \star}$ & $-.36^{\star \star \star}$ \\
\hline 11. Job Satisfaction & & $.58^{\star \star \star}$ & $-.17^{\star}$ & .16 & $-.38^{\star \star \star}$ & $-.32^{\star \star \star *}$ & $.41^{\star \star \star}$ \\
\hline 12. Affective Commitment & & & -.10 & $.37^{\star \star \star}$ & $-.31^{\star \star \star}$ & $-.26^{\star \star}$ & $.36^{\star \star \star}$ \\
\hline 13. Continuance Commitment & & & & .10 & -.14 & $.19^{\star}$ & $-.27^{\star \star}$ \\
\hline 14. Normative Commitment & & & & & -.08 & .04 & -.03 \\
\hline 15. Turnover Intentions & & & & & & .15 & -.16 \\
\hline 16. Psychological Distress & & & & & & & $-.53^{\star \star \star}$ \\
\hline 17. Life Satisfaction & & & & & & & \\
\hline
\end{tabular}

Note: ${ }^{*} p<05,{ }^{* \star} p<01,{ }^{* \star} p<001$ 
and thus was the only outcome variable that did not correlate with any of the PD dimensions. Moreover, Turnover Intentions was significantly related to only five PD dimensions but not any of the Job-Entry factors and Derogation. This means that intent to leave the organizations is mostly related to perceived discrimination during employment stage and not pre-employment. Similar to Affective and Continuance Commitment, Psychological Distress also correlated significantly with seven PD factors but not Local Requirements, Network and Differing Treatment. Finally, Life Satisfaction correlated significantly with almost all PD factors except Network.

Overall, Network did not correlate with any of the outcome variables while Local Requirements was related only to Continuance Commitment and Life Satisfaction. Thus, among the PD factors, these two factors did not correlate significantly with most outcome variables.

\section{Regression Analyses}

Separate regressions were run for each outcome variable. The outputs for seven regression analyses are shown in Table 6.3 for the job outcome variables and in Table 6.4 for psychological well-being outcomes. In every regression analysis, demographic information like age, gender (coded as $1=$ male, $0=$ female), income (coded as $1=$ above median, $0=$ below median $)$ and English Proficiency self-ratings were entered on the first step to serve as controls for their effects on the outcome measures ${ }^{4}$. To determine the incremental validity, the four Job-Entry subscales (non-local, non-recognition of education and experience, local requirements, and network) were entered in

\footnotetext{
$4 \quad$ Median annual income is $\$ 50,000$ with $52 \%$ and $48 \%$ of the sample were earning below and above the median income respectively.
} 
Table 6.3

Standardized Coefficients ( $(\beta)$ in Hierarchical Regressions for the Prediction of Organizational Outcomes

\begin{tabular}{|c|c|c|c|c|c|c|c|c|c|c|c|c|c|c|c|}
\hline \multirow[b]{2}{*}{ Predictors } & \multicolumn{3}{|c|}{$\begin{array}{c}\text { Job } \\
\text { Satisfaction } \\
\text { Step }\end{array}$} & \multicolumn{3}{|c|}{$\begin{array}{c}\text { Affective } \\
\text { Commitment } \\
\text { Step }\end{array}$} & \multicolumn{3}{|c|}{$\begin{array}{c}\text { Continuance } \\
\text { Commitment } \\
\text { Step }\end{array}$} & \multicolumn{3}{|c|}{$\begin{array}{c}\text { Normative } \\
\text { Commitment } \\
\text { Step }\end{array}$} & \multicolumn{3}{|c|}{$\begin{array}{c}\text { Turn-Over } \\
\text { Intentions } \\
\text { Step }\end{array}$} \\
\hline & 1 & 2 & 3 & 1 & 2 & 3 & 1 & 2 & 3 & 1 & 2 & 3 & 1 & 2 & 3 \\
\hline \multicolumn{16}{|l|}{ Control Variables } \\
\hline Age & .03 & -.01 & -.03 & .13 & .07 & .09 & $.28 * *$ & $.32 * *$ & $.25^{*}$ & -.05 & -.07 & .01 & $-.26^{*}$ & $-.26^{*}$ & $-.24 *$ \\
\hline Gender & .06 & .07 & .09 & -.05 & .03 & .03 & .03 & .09 & .07 & -.02 & .06 & .10 & -.03 & -.01 & -.01 \\
\hline Income & .01 & -.02 & -.15 & -.00 & -.06 & -.17 & -.14 & -.12 & -.05 & -.01 & -.07 & -.13 & -.18 & -.20 & -.14 \\
\hline English Proficiency & -.16 & -.06 & .03 & -.02 & -.01 & .03 & $.27 * *$ & .18 & .21 & .11 & .08 & .02 & -.10 & -.13 & -.22 \\
\hline \multicolumn{16}{|l|}{ Job-Entry } \\
\hline Non-Local & & $-.28 *$ & .09 & & .00 & .21 & & .16 & .15 & & .15 & .11 & & .16 & -.11 \\
\hline Non-Recog of Educ/Exp & & -.06 & .10 & & -.20 & -.12 & & $.24 *$ & .19 & & -.15 & -.12 & & -.06 & -.12 \\
\hline Local Requirement & & .02 & -.08 & & .08 & -.02 & & .00 & .09 & & -.00 & -.07 & & -.10 & .02 \\
\hline Network & & .15 & -.08 & & .17 & .13 & & .15 & .05 & & .11 & .21 & & -.05 & .08 \\
\hline \multicolumn{16}{|l|}{ On-the-Job } \\
\hline $\begin{array}{l}\text { Hindered Career Path \& } \\
\text { Uniform Treatment }\end{array}$ & & & $-.44 * * *$ & & & $-.35 *$ & & & $.27 *$ & & & -.25 & & & .15 \\
\hline Inadequate Compensation & & & $-.34 * * *$ & & & -.01 & & & -.21 & & & .18 & & & .14 \\
\hline Differing Treatment & & & $-.18 *$ & & & .00 & & & -.12 & & & -.05 & & & .18 \\
\hline Exclusion & & & -.11 & & & $-.34 *$ & & & .07 & & & .08 & & & $.27 * *$ \\
\hline Derogation & & & -.09 & & & -.00 & & & .08 & & & -.14 & & & .08 \\
\hline Work Dynamics & & & -.04 & & & .12 & & & -.11 & & & .23 & & & -.02 \\
\hline $\boldsymbol{R}^{2}$ & .03 & .12 & .62 & .02 & .08 & .25 & .17 & .28 & .33 & .02 & .05 & .11 & .11 & .13 & .34 \\
\hline$\Delta R^{2}$ & .03 & .09 & $.51 * * *$ & .02 & .06 & $.18^{* *}$ & $.17 * *$ & $.11^{* *}$ & .05 & .02 & .04 & .06 & $.11 *$ & .02 & $.21 * * *$ \\
\hline
\end{tabular}


step 2 and the six On-the-Job subscales (hindered career path \& unequal treatment, inadequate compensation, differing treatment, exclusion, derogation, and disharmonious work dynamics) were entered in the final step. The results of the six analyses are the following:

\section{$P D$ and Job satisfaction.}

In the prediction of Job Satisfaction, the overall model explained a significant amount of variance $\left(R^{2}=.62, F(14,94)=11.07, \mathrm{p}<.001\right)$. Steps 1 and 2 did not result in a statistically significant change in $R^{2}$ although NonLocal was a significant predictor in Step 2. In the final step, a significant incremental change in $R^{2}$ was observed when On-the-Job PD factors were entered into the regression equation $\left(\Delta R^{2}=.51, \Delta F(6,94)=21.06, p<.001\right)$. Upon examination of beta values, only Hindered Career Path and Unequal Treatment, Inadequate Compensation and Differing Treatment significantly predicted job satisfaction over and above the control variables and Job-Entry PD factors. Hence, in partial support to H6.1, the higher the PD (related to career advancement, compensation and organizational policies) the less satisfied the immigrants were in their jobs.

\section{$P D$ and Organizational Commitment.}

For the first dimension of organizational commitment, the overall model explained $25 \%$ of the variance in the prediction of Affective Commitment: $F(14,94)=2.28, \mathrm{p}<.05$. Hypothesis 6.2 a was partially supported with a significant incremental change in $R^{2}$ after the On-the-Job PD factors were entered in the third step $\left(\Delta R^{2}=.18, \Delta F(6,94)=3.70, \mathrm{p}<.01\right)$. Steps 1 and 2 did not result in a statistically significant change in $\mathrm{R} 2$. In the final regression step, only Hindered Career Path \& Unequal Treatment and 
Exclusion were significant predictors of Affective Commitment. This means that the higher the perceptions that there is hindrance in career advancement and the higher PD in the form of exclusion in the workplace, the less the emotional attachment to the workplace.

In the prediction of Continuance Commitment, the overall model explained $33 \%$ of the variance: $F(14,94)=3.30, \mathrm{p}<.001$. There was as significant incremental change in $R^{2}$ when Job-Entry PD factors were entered in Step $2\left(\Delta R^{2}=.11, \Delta F(4,100)=3.93, \mathrm{p}<.01\right)$ even after controlling for demographic variables. Closer inspection of the beta values in the second step revealed that only Non-Recognition of Education and Experience was a significant predictor of continuance commitment after controlling for demographic variables. However, this factor was no longer significant in Step 3. The entry of the On-the-Job PD factor in the final step did not result in a significant incremental change in $R^{2}:\left(\Delta R^{2}=.05, \Delta F(6,94)=1.17\right.$, n.s. $)$ although Hindered Career Path was significant. This means that the higher the perceptions that qualifications and experienced are not recognized, the higher the perceived cost of leaving the organization. Hypothesis $6.2 b$ is thereby rejected since the hypothesis originally predicted a positive relationship between PD and continuance commitment.

The last hypothesis on organizational commitment (H6.2c) relating to Normative Commitment was not supported. The overall regression equation for normative commitment was not statistically significant: $F(14,94)=.85$, n.s. None of the PD factors were a significant predictor of normative commitment. 


\section{$P D$ and Turnover intentions.}

The overall model explained $34 \%$ of the variance in the prediction of Turnover intentions: $F(14,95)=3.57, \mathrm{p}<.001$. The entry of On-the-Job PD factors in Step 2 did not result in a statistically significant change in $R^{2}$. Hypothesis 6.3 was partially supported with a significant incremental change in $R^{2}$ after the On-the-Job PD factors were entered in the third step $\left(\Delta R^{2}=.21\right.$, $\Delta F(6,95)=5.10, \mathrm{p}<.001)$. After the beta values were examined, however, only Exclusion was a significant predictor of Turnover Intent after controlling for age. This means that higher perceptions of being excluded in workplace interactions are associated with greater intention to leave the organization.

\section{PD and Psychological distress.}

The fourth hypothesis (H6.4) was partially supported. For the prediction of Psychological Distress, the overall model explained a significant amount of variance $\left(R^{2}=.29, F(14,93)=2.70, \mathrm{p}<.01\right)$. Steps 1 and $2 \mathrm{did}$ not result in a statistically significant change in $R^{2}$ although Non-Local was a significant predictor in Step 2. In the final step, a significant incremental change in $R^{2}$ was observed when On-the-Job PD factors were entered into the regression equation $\left(\Delta R^{2}=.14, \Delta F(6,93)=3.12, p<.01\right)$. Upon examination of beta values, only Derogation significantly predicted Psychological Distress. Hence, the higher the perceptions of being derogated in the workplace in the form of ethnic slurs or jokes are associated with higher levels of psychological distress.

\section{$P D$ and Life satisfaction.}

The last hypothesis (H6.5) was also partially supported. The overall model explained a significant amount of variance $\left(R^{2}=.26, F(14,94)=2.41\right.$, 
Table 6.4

Standardized Coefficients ( $\beta$ ) in Hierarchical Regressions for the Prediction of Psychological Well-being Outcomes

\begin{tabular}{|c|c|c|c|c|c|c|}
\hline \multirow[b]{2}{*}{ Predictors } & \multicolumn{3}{|c|}{$\begin{array}{l}\text { Psychological } \\
\text { Distress } \\
\text { Step }\end{array}$} & \multicolumn{3}{|c|}{$\begin{array}{c}\text { Life } \\
\text { Satisfaction } \\
\text { Step }\end{array}$} \\
\hline & 1 & 2 & 3 & 1 & 2 & 3 \\
\hline \multicolumn{7}{|l|}{ Control Variables } \\
\hline Age & -.07 & -.03 & -.08 & .07 & .03 & .07 \\
\hline Gender & -.02 & .07 & .05 & .09 & .04 & .05 \\
\hline Income & -.12 & -.16 & -.10 & .07 & .08 & .06 \\
\hline English Proficiency & .19 & .14 & .13 & -.16 & -.07 & -.03 \\
\hline \multicolumn{7}{|l|}{ Job-Entry } \\
\hline Non-Local & & $.27 *$ & .11 & & $-.33^{*}$ & -.15 \\
\hline Non-Recog of Educ/Exp & & .06 & -.06 & & -.05 & .04 \\
\hline Local Requirement & & -.20 & -.11 & & .09 & .02 \\
\hline Network & & .13 & .16 & & .01 & -.03 \\
\hline On-the-Job & & & & & & \\
\hline $\begin{array}{c}\text { Hindered Career Path \& } \\
\text { Unequal Treatment }\end{array}$ & & & .21 & & & -.09 \\
\hline Inadequate Compensation & & & .09 & & & -.08 \\
\hline $\begin{array}{l}\text { Disharmonious Work } \\
\text { Dynamics }\end{array}$ & & & .05 & & & -18 \\
\hline Differing Treatment & & & -.15 & & & .06 \\
\hline Exclusion & & & .10 & & & .01 \\
\hline Derogation & & & $.24 *$ & & & $-.27 * *$ \\
\hline$R^{2}$ & .07 & .15 & .29 & .05 & .13 & .26 \\
\hline$\Delta \boldsymbol{R}^{2}$ & .07 & .08 & $.14^{* *}$ & .05 & .09 & $.13^{*}$ \\
\hline
\end{tabular}

$\mathrm{p}<.01)$ in the prediction of Life Satisfaction. Steps 1 and 2 did not result in a statistically significant change in $R^{2}$ although Non-Local was a significant predictor in Step 2. In the final step, a significant incremental change in $R^{2}$ was observed when On-the-Job PD factors were entered into the regression equation $\left(\Delta R^{2}=.13, \Delta F(6,94)=2.80, p<.05\right)$. Upon examination of beta values, only Derogation significantly predicted Life Satisfaction. Hence, the higher the perceptions of being derogated in the workplace in the form of ethnic slurs or jokes, the less the life satisfaction. 
Overall, five significant effects (please see Figure 6.1) were found in the prediction of outcomes using PD factors. Among the job-entry factors, NonLocal displayed significant beta values when entered in the $2^{\text {nd }}$ Step of Job Satisfaction, Psychological Distress and Life Satisfaction. However, on-thejob factors that showed significant beta values in separate regression analyses except for Normative Commitment. Consequently, the incremental validity of the on-the-job factors was established separately in the prediction of six variables out of seven in this study. Five hypotheses were supported (job satisfaction, affective commitment, turnover intention, psychological distress and life satisfaction) and two were rejected (continuance commitment and normative commitment).

\section{Discussion}

The overall assumption of the present study is that PD during preemployment and employment of immigrants' working experiences would have effects on their job and psychological health outcomes over and above other demographic factors like age, gender, income and language proficiency. While previous workplace discrimination studies have examined PD of minority and majority groups in terms of gender and ethnicity (e.g. Ensher, 2001; Sanchez \& Brock, 1996, Foley et al., Gutek et al., 1996), this study also considered first generation immigrants thereby generating perceptions from participants with multiple-demographic characteristics. Moreover, the prediction of organizational and psychological well-being variables as outcomes of PD has not been investigated in one model. Thus, the unique contribution of this study is to look into the resulting outcomes of PD for immigrants in the workplace over and above their demographic and ascribed characteristics. 
Figure 6.1

Summary of Significant Findings between PD and Outcomes

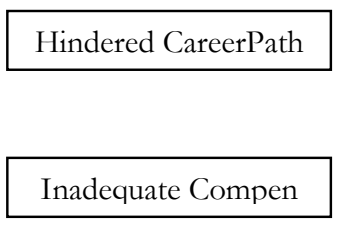

Differing Treatment

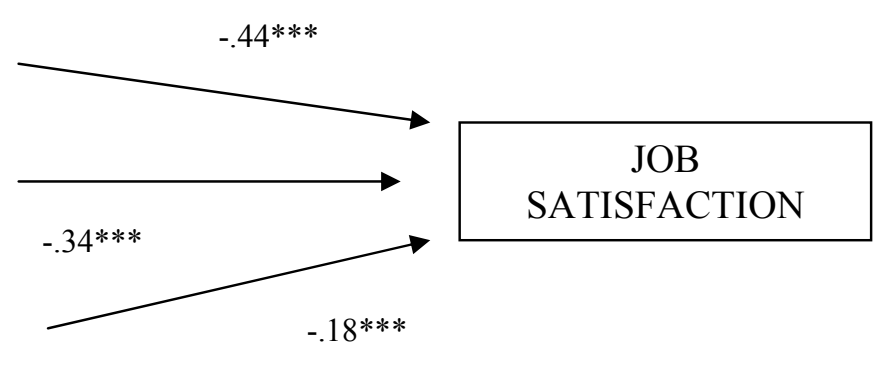

$-.34 *$
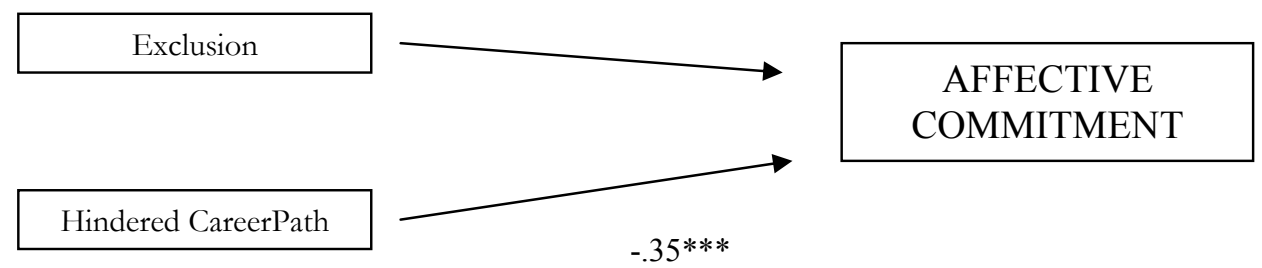

Hindered CareerPath

$-.35 * * *$

Non-recognition of Educ \& Experience

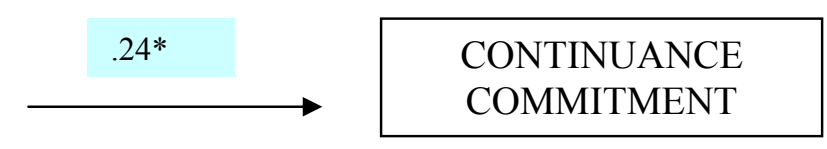

$.27 * *$
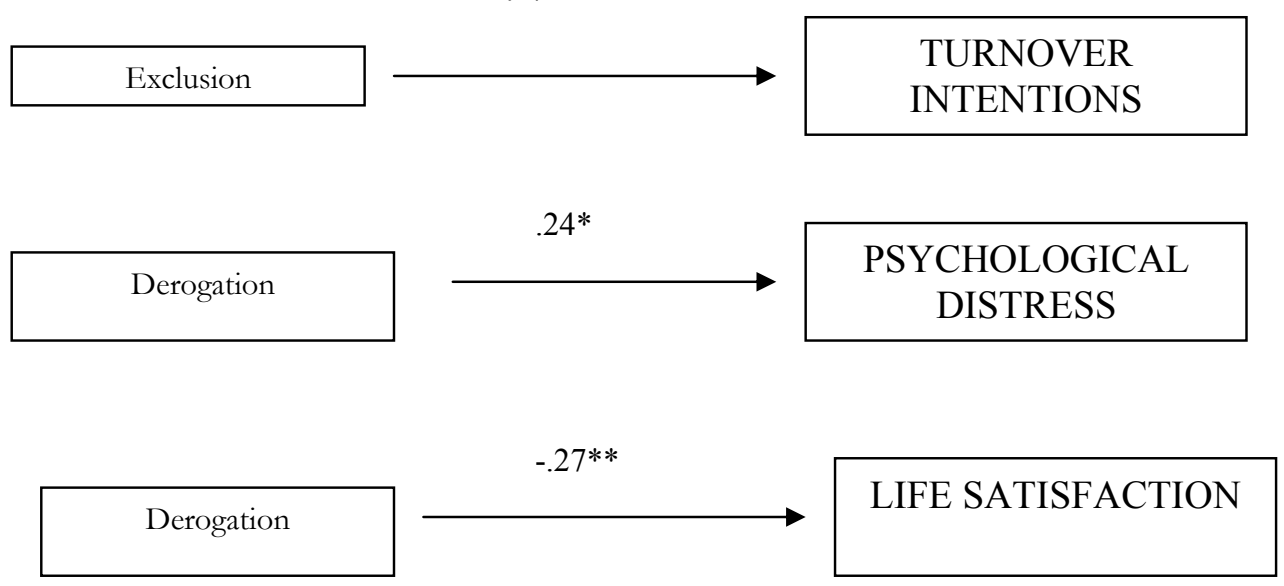
addition of the Job-Entry PD factors in these outcomes at Step 2 did not result in incremental change in $R^{2}$. The best predictors of the outcomes are the six

Regression analyses of the incremental effects of On-the-Job PD on job satisfaction, affective commitment, turnover intentions, psychological distress and life satisfaction partially supported the proposed hypotheses. Incremental effects of Job-Entry PD factor for continuance commitment, on the other hand, were also significant although the direction of the relationship was not as expected thereby rejecting the proposed hypothesis. No significant incremental effects were found for normative commitment.

Based on these results, there are two key findings (elaborated in the next sections) leading to other important sub-findings, each adding to the better understanding on the extent of the consequences brought about by immigrants' perceptions.

\section{Pre-Employment Perceptions (Job-Entry) and Outcomes}

The first key finding is that PD during the pre-employment stage or job hunting stage has no lasting effect for the immigrants in relation to their outcomes except for Continuance Commitment. The general findings are consistent with the belief that immigrants go through stages in their resettlement, which include passing through initial stressful situations which they eventually get over with in the long run (Pernice \& Brook, 1996; Scott \& Scott; 1989). However, one unexpected result relate to Non-recognition of Education and Experience (perceiving that qualifications and work experience are not recognized), which increases commitment to the workplace due to perceived cost associated with leaving the organization. 
In the commitment literature, continuance commitment was found to have two interpretable factors that are negatively correlated namely, 'perceived sacrifice' and 'lack of alternatives' (McGee \& Ford, 1987). These two subcomponents were found to be highly correlated and relate negatively with affective and normative commitment (Meyer, Stanley, Herscovitch, \& Topolnytsky, 2003). Hence, the component that may be interacting with the PD factor (Non-recognition of education and experience) is the 'lack of alternatives' component. Therefore, immigrants who view that their qualifications do not mean much in New Zealand will have higher attachment to their organization, thinking that it would be unwise to leave their organization since there are no clear alternative employment opportunities outside their current employer. Other employers may similarly treat them by ignoring their qualifications obtained outside New Zealand. Hence, the respondents may find the cost of leaving the organization very high since there is no assurance of another employment.

On the other hand, it has been noted that continuance commitment would demonstrate mixed results in its relationships with its predictors and consequences such that some of the relationships were not in the predicted direction (Dunham, Grube, \& Castaneda, 1994). Hence, continuance commitment has been previously criticized for its construct validity as being problematic (Ko, Price \& Mueller, 1997). Consistent with this, the metaanalysis by Fischer and Mansell (2009) found both negative and positive relationships between continuance commitment and uncertainty avoidance, as 
measured by Hofstede and $\mathrm{GLOBE}^{5}$ respectively. Thus, the negative direction between the relationship of the PD factor and continuance commitment could be another area that needed further investigation since this study's findings also showed inverse relationship to what was originally expected.

\section{During Employment (On-the-Job) Perceptions and Outcomes}

The second key finding is that PD during employment (On-the-Job PD factors) has far reaching effects on immigrants' job attitudes and psychological health. For instance, lower job satisfaction was found to be predicted by several On-the-Job PD factors namely, higher PD in career advancement, higher PD in wages and benefits, and higher PD in overall organizational policies. Thus, the findings of this study are consistent with the intrinsic facet of job satisfaction wherein PD related to low compensation causes job dissatisfaction (Judge et al., 2001). Similarly, perceiving that there are policies that are discriminatory to one's ethnicity or background also result in less job satisfaction. These organizational policies may be related to human resource structures that hinder advancement, pay and benefits - all of which are also categorized under the intrinsic facet of job satisfaction (Judge et al., 2001).

Moreover, higher perceptions of being discriminated in career advancement were also related to less emotional attachment to organizations. This is consistent to earlier findings that appropriate implementation of human resource practices related to career development like promotional procedures can increase emotional attachment to organizations especially if the

\footnotetext{
${ }^{5}$ GLOBE - (Global Leadership and Organizational Behavior Effectiveness) project research conducted in 62 countries.
} 
organization is perceived as equally supportive to them (Meyer \& Smith, 2000).

Another significant result of this study is that Exclusion predicts lower affective commitment and higher turnover intention. Thus, those who feel that they are excluded in work-related social interactions feel less emotionally attached to their organizations and have higher intentions to leave. Ensher et al. (2001) found that higher PD (from supervisors) predicted less commitment to organizations. This could help explain how interactions with people at work could affect an individual's levels of commitment. Moreover, perceiving that you are being excluded results in poor workplace interactions that eventually lead to propensity to leave the organization. In previous studies, it has been found that low commitment to organization is related to higher turnover intention and actual turnover behaviour (Chen \& Francesco, 2000; Konovsky \& Cropanzano, 1991; Meyer et al., 1993). Among the three dimensions of organizational commitment, the emotional attachment to the organization has also been found as the best predictor of attitudes and behaviours in organizations including turnover intentions (Feather \& Rauter, 2004; Wong et al., 2002). Thus, the findings of the current study are consistent with previous studies demonstrating the negative relationship of PD with emotional attachment to organizations and the positive relationship with intent to leave.

Among the three commitment dimensions, only Normative Commitment was not predicted by either Job-entry PD factors nor the On-theJob PD factors. Although normative commitment showed significant positive correlation with affective commitment, the PD factors did not contribute 
uniquely to the prediction of normative commitment as they did with affective commitment.

Normative commitment did not relate significantly to most variables in study. The reason could be that most variables in study are attitudinal in nature and normative commitment has been shown to be more related to employee behaviours specifically longstanding behaviour patterns (Yao \& Wang, 2006). Moreover, it has been pointed out that the role of normative commitment in relation to work-related variables could vary across cultures. In Meyer et al. (2002) meta-analysis, they found that normative commitment relate more strongly to employee behaviours in studies that were conducted outside of North America as compared to studies done within North America. Fischer \& Mansell's (2009) meta-analysis also found that normative commitment showed a stronger relationship with turnover intentions in collectivist samples.

Based on the above-mentioned studies, it could be expected that the results would show stronger relationship between normative commitment and other variables since the sample in this study are mostly coming from Asian countries (with a high concentration of Filipino respondents). However, it appears that in the New Zealand workplace context, the concept of "being obligated" or being loyal to the organization has not been deeply internalized yet at least among the participants. Thus, immigrants' appears to have lower levels of normative pressures to be committed to the organization. Thereby, this weakens the link to other variables. Recent immigrants are noted to have moved in to New Zealand mostly for economic reasons. Thus, their personal objective would perhaps look for an organization that could answer their economic needs and ensure their success financially. Hence, moral obligations 
to remain in an organization would come in the long-term if indeed the organization has proven to have answered their needs.

Alternatively, the meta-analysis of Fischer and Mansell (2009) also found that power distance ${ }^{6}$ is positive related with normative commitment. This means that in hierarchical contexts, normative pressures to stay in the organization are stronger since observation of social norms is valued. The findings of the focus groups discussion, however, revealed the New Zealand workplace context as egalitarian. Hence, this may be another factor that is affecting the perceptions about obligatory pressures to remain in the organization in this study. More in-depth future studies are warranted to ascertain the nature of the findings on normative commitment in relation to PD among immigrants.

Finally, the perceptions that immigrants' ethnic background is being derogated or treated with less respect have far reaching effects on psychological well-being, leading to higher psychological distress and less satisfaction in life. These findings are consistent with studies that have focused on the negative impact of PD on the psychological and physical well-being of target groups. General findings show that negative treatment and undesirable work atmosphere lead to ill-effects on health and well-being (e.g Bowman, 1991; Jasinskaja-Lahti, et al., 2007; Pernice, et al., 2000)

These findings pose as a challenge to management and policy making bodies for how to best approach the improvement of formal and informal structures in the workplace to address discriminatory perceptions. Moreover, the results of the study should therefore alert the policy making bodies and

\footnotetext{
${ }^{6}$ Hofstede (1980) refers to power distance as the extent to which inequality and power differentials are acceptable in a society.
} 
employers on the issues that immigrant employees face, as a function of their status as immigrants on top of other demographic characteristics that are different from the majority. Since PD has incremental effects in both organizational attitudes and psychological well-being, these outcomes should be given ample focus in setting up or improving diversity management programs in organizations.

\section{Limitations of the Study}

The findings of this study were found to be consistent with the general literature of discrimination and also provide new additions to the growing empirical discrimination studies with the inclusion of immigrant employees as participants. Nonetheless, it is acknowledged that there are also methodological limitations. First, the sensitive nature of the questions may be a cause for social desirability bias. However, this limitation was addressed in

part by the guaranteed confidentiality of responses as well as anonymity of participants.

Second, all of the variables were assessed by self-report measures, raising the possibility of common methods bias. However, PD is basically a subjective experience and is hard to assess objectively.

Third, causal relationships were inferred although this study was crosssectional and did not capture immigrant perceptions within organizations. Thus, it is imperative to note that causal inferences made from cross-sectional designs are never more than inferences (Moorman, 1991).

Fourth, there may be omitted variables that might reflect a more complex process than what was depicted in the regression modes. However, due to the novelty of the way that the variables were selected to fit the study's 
framework, selection of covariates were limited and based on what is currently salient in the literature on discrimination.

Fifth, the majority of the sample are Asian Immigrants specifically Filipino which is not reflective of the actual immigrant ethnic composition in New Zealand. Thus generalization of findings can not be readily attempted.

Lastly, the viewpoints of non-immigrants employees, management, or non-working immigrants were not assessed. Hence, there may be also some aspects that have been missed in the interpretation of the discrimination model.

\section{Suggestions for Future Research}

There are several avenues for future research that were identified in this study. First, the sample was restricted to first generation immigrant employees. Hence, results should not be generalized beyond the population from which they were obtained. Future studies are needed to cross-validate the results with a more diverse ethnic population, a larger immigrant sample, or a different set of organizational contexts and occupations. Second, the dependent variables consisted mostly of attitudes rather than behaviours. Thus, there is a need to include more behavioural consequences of perceived discrimination to examine the full ramifications of immigrants' perceptions. These behavioural measures could include grievance reports, harassment reports, actual turnover data, leaves of absence and the like.

Third, it is important to identify the casual mechanisms of the reported relationships through the use of longitudinal studies. These longitudinal studies could examine the short- and long-term consequences of exposures to discrimination on the overall health and well-being of immigrants. 
Fourth, research that identifies with greater specificity how social status factors such as levels of education, age, and types of occupations, length of stay and other variables modify exposures to health-debilitating employment-related discriminations is needed.

Fifth, research is also needed that compares immigrant employees' perceptions of discrimination with that of local employees to determine if there are similarities/differences in perceptions and resulting outcomes. Comparative studies between minority and majority groups will be helpful in assessing if the bases of discriminatory perceptions are systemic or minority group targeted.

Lastly, further studies are needed to assess whether and to what extent the theoretical framework developed in this study will hold considering other variables that have been linked to the outcomes in study. For instance, what will be the incremental effects of PD on the outcomes if empirically robust variables such as justice perceptions are considered in the analyses?

\section{Summary and Conclusions}

This study explored the extent to which PD factors could predict selected attitudinal and psychological well-being outcomes. In summary, the immigrants' outcomes in relation to PD are: Job satisfaction was solely predicted by PD related to work conditions. Affective commitment, on the other hand, was predicted by both PD on work conditions and quality of relationships at work. Continuance commitment was positively associated with recognition of qualifications and experience. Turnover intentions were predicted by perceptions of exclusion. Lastly, psychological well-being 
outcomes such as psychological distress and less satisfaction in life were both predicted by perceptions of being derogated in the workplace. Thus, the outcomes of PD depend either on systemic or relational aspects.

In conclusion, this study provided evidence of the continuing existence of PD among first generation immigrants in New Zealand, as well as a relationship between their PD in two aspects of the employment process (job entry and on-the-job) and important outcomes related to job satisfaction, commitment to their organization, intentions to leave, mental health and general satisfaction in life. As part of a growing body of research on ethnicityrelated discrimination, this study broadens our understanding of the influence of PD on organizational processes by providing a theoretical framework grounded in theoretical models that have linked human cognitions to resulting attitudes and behaviours. The next chapter will address the other portion of the research framework that is looking at the possible antecedents of PD. The final study is intended to complete the antecedent-outcome distinction of the PD process. 


\section{Chapter 7}

ANTECEDENTS OF PERCEIVED DISCRIMINATION (STUDY 4)

\section{Introduction}

This chapter focuses on the antecedents of employees' perceived discrimination (PD) in the workplace or the "upstream" analysis (Schnittker \& McLeod, 2005) of the process of perceived discrimination. As part of the major objectives of this thesis, this study will investigate what factors influence workplace discriminatory perceptions at the individual level and determine which of these factors are more salient than others. In light of the saying that it is more difficult to swim upstream than downstream, a careful investigation of these factors or antecedents will be done through a larger and ethnically diverse sample of employees coming from different national backgrounds and having a mix of immigrants and New Zealand-born employees.

The pattern of cultural differences between locally born and overseas born peoples in New Zealand is rather interesting. Aside from the overseasborn population (or the first generation immigrants), the native-born population is also a mix of individuals of foreign ancestry from UK and other European countries, as well from as various Pacific Islands, Australia and Asia. As presented in Chapter 4, New Zealand has historically been an immigrant country (Brosnan, 1988). Massive waves of British and Irish settlers have joined the indigenous Maori inhabitants in the 1800's, followed by the Pacific Peoples in the 1960's and more recently people from nontraditional sources like Asia in the 1990's (Coates \& Carr, 2005). Thus, New 
Zealand-born nationals or New Zealanders may also be attributing their background to different ethnicities. The last two succeeding national censuses allowed the reporting of more than one ethnicity if one sees fit (Statistics New Zealand, 2003; 2006) which shows an affirmation of a high degree of cultural mixing within New Zealand's 4.3 million population. Thus, a re-categorization may be possible in terms of visible immigrants (non-European or nonCaucasian), non-visible (European or Caucasian) immigrants, visible nativeborn (with non-European or non-Caucasian heritage) and non-visible native born (with European or Caucasian heritage).

Riordan et al. (2005) called for future research that simultaneously examines majority and minority perceptions and how are they related to real or perceived discrimination. The pattern of differences between minority and majority perceptions are yet to be studied in detail. Study findings show that minority groups continue to experience discrimination despite legal and social pressures (Dipboye \& Collela, 2005). Other studies show that majority group may perceive less discrimination than the minority group. In certain circumstances like in the presence of affirmative action (AA) policies, majority group members may report less support for these policies (Konrad \& Spitz, 2003) since they perceive these policies as perpetuating a new breed of discriminatory practices.

Majority members are less likely to believe that discrimination exists as reflected in racism and gender literature (Bobo \& Kluegel, 1993: Konrad \& Hartmann, 2001; Kravitzs \& Klineberg, 2000; Kravitz, Klineberg, Avery, Nguyen, Lund, \& Fu, 2000; Tougas \& Beaton, 1993). Findings have been attributed to the belief of privileged groups that their status was rightfully 
theirs through their own merit thus making them unaware of their unjustified advantages (Jacques, 1997). Moreover, members of majority groups usually perceive less discrimination since their values are generally congruent to the value system of the organization. In the US, individualism, self-reliance, work ethic, obedience and discipline are the usual central values of the culture (Katz \& Hass, 1988; Kinder \& Sears, 1981). If members of the majority group violate these central values, it is only then that they will feel being discriminated. For instance, being a White obese person will be attributed to the individual as lacking in discipline or lacking in self control (Crandall, 1994). Perceived discrimination by members of the minority groups, on the other hand, come from unfair treatment due to their demographic and ascribed characteristics that have nothing to do with the value systems in the organizations. This situation is aptly described by Schneider, Swan, \& Fitzgerald, (1997) with the questions 'were you left out?' and 'were you left out because you are Black?'

Thus, this study attempts to clarify the issues between the differing perceptions of the majority group (New Zealand-born or NZ-Born) and minority group (immigrants) employees in the workplace and see if the country of birth or their birthplace is a salient factor in discrimination. To address these issues, the current study considered a sample of employees with a mix of NZ-born and immigrants. They have two distinct statuses in terms of birthplace but can achieve a super-ordinate identity as "employees". Generally, employees face an array of challenges in the workplace regardless of race or ethnicity, age, gender and so on thus making them all susceptible to discrimination. However, on top of being employees, there is the additional 
status of being a member of the minority group that may reflect higher negative perceptions than the local New Zealanders.

Thus, the study attempts to address the key question: What factors are more salient as antecedents of employees' PD in the organizational context? In relation to this key question, the overall goal of the present study is to contribute to the current literature on PD in the workplace by investigating a number of personal and organizational variables as antecedents of perceived discrimination and testing the tenability of new PD measures. Specifically, the first objective is to test the new PD measures by first administering the scales on a diverse sample of employees and determining its psychometric properties. The new PD scales will be used to attain the second objective, which is to investigate the antecedents of PD and determine whether particular variables are more salient as antecedents in perceived discrimination.

There are four major sections in this chapter. The first section is the review of related literature on antecedents of PD. The second section is the method section that includes the steps taken to construct PD scales that are applicable to employees regardless of country of birth (i.e. native-born versus overseas born). Measurement invariance has become a topical concern within the last decade in cross cultural research (Cheung \& Rensvold, 2002; Steenkamp \& Baumgartner, 1998) since it is important for scholars to ensure that scales items have the same meaning for members of different cultures (e.g. Black Americans vs. Latino Americans). In my review of existing raceor ethnicity-related measurements of PD in Chapter 4, I did not encounter any steps taken by authors to test measurement invariance. Thus, my intention of constructing PD scales that are structurally and metrically applicable to 
different groups is an important contribution to the literature. To come up with these measures, the IPDWS-P items in Study 3 (from Chapter 5) were initially modified to make them applicable to the two biggest groups in the sample of this study - native-born and overseas-born employees. Then, tests were conducted to ensure that the new scales used for Study 4 have the same meanings for both groups. I will then present the central results of this chapter, namely the empirical investigation of the antecedents of PD in the workplace. The fourth and final section is the general discussion of the results, including the limitations of the study, suggestions for future research and conclusion.

\section{Factors Affecting Perceived Discrimination in the Workplace}

As introduced in Chapter 3, the antecedents of PD (see left-hand portion of Figure 3.1 of Chapter 3) are assumed to influence how an employee perceives events as discriminatory. In my proposed PD framework, it is assumed that an individual's appraisal of events as discriminatory or an environment as perpetuating discrimination largely depends on the perceiver's inherent and socially ascribed characteristics and how his/her surrounding environment has institutionalized discriminatory practices. The antecedents are further subdivided into proximal influences and distal influences. First, the proximal antecedents are the inherent or ascribed characteristics of the targets of discrimination. These factors are internal to the perceiver such as demographic characteristics including country of birth, ethnicity, gender, age and income. The second categorization of antecedents is the distal antecedents or contextual factors. These factors are more contextual and external to the individual yet they influence how targets perceive discrimination. In the context of workplace discrimination, the factors that will be considered as 
distal factors are organizational characteristics and employee perceptions of the processes and structures within the workplace or organization.

\section{Proximal Influences}

In real life situations, incidence of discrimination is hard to determine because the perpetrators play a part in the discrimination process (Phinney et al., 1998). Thus, targets or victims of discrimination like minorities and immigrants face uncertainties about what causes the discriminatory acts because the reason for the actions are usually unclear to them (Lazarus \& Folkman, 1984). Therefore, discrimination may be perceived as absent although discriminatory practices were applied or it can be perceived even though it did not happen (Phinney et al., 1998). In the event of ambiguous situations such as discrimination, individuals make attributions to understand the situation. Experimental studies (see Crocker, Voelkl, Testa, \& Major, 1991; Ruggiero \& Taylor, 1995) show that participants attributed negative events to discrimination in conditions of low ambiguity or when making discrimination more obvious to participants. However, in conditions of high ambiguity, the participants attributed the negative events to the self. In contrast to experimental studies, external factors (e.g. perpetrator's characteristics or organizational characteristics) are hard to manipulate in nonexperimental settings thus individual differences are deemed more important to examine in terms of perceptions (Phinney et al., 1998).

The meta-analysis in Chapter 2 discussed several theoretical frameworks that could best explain how individual differences like personal characteristics could influence PD. The theoretical frameworks are categorized into two approaches (Phinney et al., 1998) - the socio-cultural perspective that 
puts emphasis on demographics characteristics of individuals, and the attributional perspective that focuses more on psychological differences of individuals. Social identity (Tajfel \& Turner, 1979; 1986) and selfcategorization theories (Turner et al., 1987) and their derivative models like Relational Demography Theory (Tsui et al., 1992), follow the socio-cultural perspective thereby giving more importance to demographic variables such as race/ethnicity, age, gender, and socio-economic status as relevant antecedents of PD. On the other hand, the attributional perspective emphasizes more stable personality characteristics of individuals as factors that partly determine whether events will be perceived as discriminatory (Lazarus \& Folkman, 1984). Most studies on self-esteem and self-mastery generally follow the attributional perspective.

The examination of the antecedents of PD in this study generally follows the socio-cultural perspective since demographic characteristic will be the main focus for proximal antecedents. In this study I will specifically examine the effect of ethnicity (having White European heritage vs. NonWhites); birthplace (immigrant status vs. NZ-born), age, gender, and income on PD in the workplace. Although different grounds for discrimination such as race/ethnicity, age, gender, immigrant status, and socio-economic status have been directly linked to outcomes in various fields of social psychology, acculturation and organizational psychology, the investigation of the relationship of these demographic factors to perceived discrimination as a form of an immediate outcome is still in its infancy (Riordan et al., 2005). 


\section{Race/Ethnicity.}

Race and/or ethnicity related issues have been central in discrimination research due to persisting disparities observed among ethnic/racial groups categorized as Whites vs. Non-Whites, or majority groups vs. minority groups despite legal pressures. In the United Kingdom (UK), non-Whites are reported to have higher unemployment rates, lower earnings and lower occupational attainment (Blackaby, Leslie, Murphy, \& O’Leary, 1998; 1999). Even for ethnic minority youth, undergoing ethnic discrimination is common in the UK (Karlsen \& Nazroo, 2002; Virdee, 1995).

In the Canadian context (Banerjee, 2008), minority groups are further categorized into visible minorities (non-White or non-Caucasians like Blacks, Middle Eastern and Asians) and the non-visible minorities (those with Caucasian heritage mostly from Europe and North America). Visible minorities were more likely to report discrimination than White ethnic groups (Dion \& Kawakami, 1996) and they feel more discriminated in the areas of job application especially in government positions, wages and career advancement (Dion, 1989). Reitz and Banerjee (2007) investigated the perceived discrimination in the past 5 years and found that $40 \%$ of the visible minorities reported discrimination including approximately 50\% of Blacks reporting discrimination while only $10 \%$ of White respondents perceived discrimination for the 5-year duration. The ICSEY study also reported that among the participants, males and the most visible minority youth reported the highest levels of perceived discrimination (Phinney et al., 2006).

Historically, Black and White differentiation is well-defined and enforced in United States legislation and practice (Butterfield, 2004). Thus, in 
the United States' discrimination literature, studies focus on this differentiation to highlight the differences in outcomes of the two groups. Among these studies, findings show that Black vs. White differences are evident in informal daily interactions at work such that Blacks perceive the workplace as less welcoming than Whites, get fewer mentors to guide them, and experience greater isolation from pertinent informal networks (Reskin, 1998). Deitch et al. (2003) studied everyday workplace discrimination and found that Black American employees have higher perceptions of daily unfair mistreatment at work compared to White Employees. In a nationwide study of full-time psychology faculty members, African Americans, Hispanic and Asian American participants reported more discrimination than White faculty members, especially those who were the only minority group member in the department (Dovidio, Gaertner, Niemann \& Snider, 2001). Based on these findings, I propose the following hypothesis:

Hypothesis 7.1: Non-White participants will report higher perceptions of discrimination in the workplace compared to White majority participants.

\section{Birthplace.}

Simultaneous with racial/ethnic differences, this study will also look in to birthplace or country of birth as a factor that can influence perceived discrimination in the workplace. The classifications for country of birth in this study will be used to categorize individuals as either born in New Zealand or born overseas (immigrants). Similar to ethnic minorities, immigrant groups have characteristics that serve as bases for their exclusion such as skin colour, language differences, physical features and cultural traits and behaviours that 
are different from the majority groups or the native-born group (Phinney et al., 1998; Westhoff, 1993). In the context of immigrants in organization, immigrants are considered as "economic threat" (Cohen, 1996) since they are regarded as rivals for job vacancies and coveted positions.

At the individual level, immigrants face challenges in the host country like culture shock (Ward et al., 2001), discrimination (IOM, 2005; Ward \& Leong, 2006), cheap labor (Marsella \& Ring, 2003), underemployment (Slack \& Jensen, 2002; Ward \& Masgoret, 2007), acculturative stress (Berry, 2006) and social exclusion (IOM, 2005).

Although race and ethnicity issues are widely researched, research on the impact of birthplace (native-born vs. immigrants) on PD and resulting organizational outcomes is almost non-existent in the organizational psychology literature (except Banerjee, 2008), regardless of the acknowledgement that workplace experiences of minorities differs in significant ways to that of the majority (Crocker et al., 1999; Evans \& Herr, 1994, Riordan \& Shore, 1997; Tsui et al., 1992). What is common in the literature is the focus on immigrant acculturation strategies and health outcomes. In some studies, immigrants' demographics like income, rates of unemployment or underemployment were considered to gauge their psychological and health outcomes.

A study that looked into psychological variables alluding to employment discrimination from the immigrants' perspective is the study by Mace, Atkins, Fletcher and Carr (2005). They found that immigrants' satisfaction in their current career was related to favourable experiences in 
pre-interview and job-seeking experiences ${ }^{7}$. Moreover, immigrants continue to face disparities in economic terms such as wages even after living in the host country for 30 years (Banerjee, 2008). Studies show that Canadian immigrants' wages are less than the wages of the Canadian native-born over their lifetime (Aydemir \& Skuterud, 2005; Bloom et al., 1995; Li, 2003; Reitz, 2001). Challenges in the workplace that are more applicable to immigrants than employees in general like wage disparities, underemployment, nonrecognition of their qualifications (IOM, 2005; Li, 2001; Slack \& Jensen, 2002; Ward \& Leong, 2006; Ward \& Masgoret, 2007) can lead to frustration and higher perceptions of discrimination, thus the following hypothesis:

Hypothesis 7.2: Immigrant employees will perceive higher discrimination than native-born employees.

\section{Gender.}

More women attribute discrimination to their gender than men (Elsass \& Graves, 1997; Wharton, 1992). Correspondingly, it is recognized in gender studies that women (Araujo \& Borrell, 2006; Konrad \& Spitz, 2003; Gutek, Cohen \& Tsui, 1996; James et al., 1994; Pavalco et al., 2003) are subjected to more workplace discrimination than men. Specifically, it has been suggested in studies that women in US organizations are hindered by a "glass ceiling" described as an invisible and impenetrable barrier in terms of advancement in their professional and managerial careers (Morrison, White \& Van Velsor, 1987). Based on the results of previous studies, it shows that females face

\footnotetext{
$7 \quad$ "Satisfaction in the current career" was used as an indirect measure of perceptions of full-employment
} 
higher probability of being exposed to discrimination thereby leading to the following hypothesis:

Hypothesis 7.3: Female will report higher perceptions of discrimination in the workplace compared to males.

Increasingly, more studies are looking into the joint effects of gender and ethnicity. One prominent theory in organizational research is the Double Jeopardy Hypothesis. To reiterate (see also chapter 3), the Double-Jeopardy Hypothesis suggests that ethnicity and gender have joint effects in influencing perceived discrimination such that minority women will experience or perceive more discrimination than minority men (Canales, 1997; Rakow \& Wackwitz, 1998). Thus, minority groups especially women can be potential targets for "multiple discrimination" (Ghosh, 1984; Moghaddam \& Taylor, 1987; Taylor et al., 1990).

Supporting evidence has been found in studies with African American women (Wilcox, 1997; Weinberg, 1998) and Hispanic women (Foley, Kidder \& Powell, 2002). However, various studies have also shown little or no support for the Double-Jeopardy Hypothesis (Dion \& Kawakami, 1996; Foley et al., 2002; Phinney et al., 1998; Verkuyten \& Nekuee, 1999; Verkuyten \& Thijs, 2001; Wilcox, 1997; Weinberg, 1998), with more recent studies reporting that females perceived less discrimination than males in a sample of ethnic minority youth in the Netherlands (Verkuyten \& Thijs, 2001) and Indian ethnic minority youth in the UK (Cassidy, 2005). Due to the inconclusive findings regarding the salience of joint effects of ethnicity and gender, this study will pose a research question instead of a hypothesis: 
Research Question 7.1: Will female minorities perceive more discrimination compared to White males, minority males and White females?

Moreover, there is a gap in the social psychology and organizational research that I would like to address in this study. This is the examination of factors beyond the two usual group memberships (i.e. ethnicity and gender). It has been pointed out that minorities, especially minority women, can be potential targets for "multiple discrimination" (Ghosh, 1984; Moghaddam \& Taylor, 1987; Taylor, et al., 1990). The immigrant status is believed to be another salient factor in discrimination perceptions. Thus, examination of the demographic differences in terms of ethnicity, gender and country of birth (immigrant status) is a significant contribution to the growing literature focusing on "multiple disadvantages" faced by immigrants in the society in general. More importantly, this triple-demographic effect is a novel contribution to the organizational literature since it has not been clearly addressed in the existing multiple group membership literature in organizational settings and is worth exploring in this current research. In the absence of relevant studies exploring the above-mentioned variables combined in one model, research questions are proposed:

Research Question 7.2: Will non-White employees who were born outside of New Zealand (NZ) perceive more discrimination compared to White NZ-born employees?

Research Question 7.3: Will non-White immigrant female employees perceive more discrimination compared to White NZ-born males, nonWhite immigrant males and White NZ-born females? 
Age.

Discrimination due to age is believed to be applicable to all individuals and the risk factor fluctuates across one's life span (Gee et al., 2007). It is also assumed that workplaces are contexts of discrimination since organizations are age-graded such that organizational roles, policy and norms vary with employee's age (Giles \& Reid, 2005). Hence in organizations, increasing attention is being accorded to age discrimination since findings show that age is more salient than ethnicity or gender in some discriminatory situations (Sigelman \& Sigelman, 1982).

Similarly, PD has been found to vary with age since some age groups are considered at high risk for exposure to discrimination (Giles \& Reid, 2005). Studies show that older adults (De Castro et al., 2008; Gee et al., 2007) encounter more workplace discrimination. Other studies show that both the youth and older employees are more likely to be discriminated by employers and by people in general (Johnson \& Neumark, 1997; Nelson, 1995). In support to these findings, studies show that mid 30's to mid 40's are the preferred age group in occupations. Specifically, McGoldrick and Arrowsmith (2001) found that $22 \%$ of job advertisements in the UK have an age limit of 37. Even college students would feel more comfortable with doctors aged 39 and bus drivers aged 40 (Kalavar, 2001). The resulting outcomes of these perceptions are equivalent to the findings of studies linking older age to limited job opportunities including pay inequities and barriers to employment (Chan \& Stevens, 2001; Hirsch, Macpherson \& Hardy, 2000). Based on these studies, the following hypothesis is proposed: 
Hypothesis 7.4: Older employees will perceive higher discrimination than younger employees.

\section{Income.}

Social Economic Status (SES) is central to the analysis of economic indicators of the majority and minority comparisons among racial/ethnic groups, gender groups, immigrants and local-born, and age groups. In some studies, SES is collective measurement of personal income, educational attainment, occupation, parents' income due to the monetary advantages as well as higher social status affiliations (Schnittker \& McLeod, 2005). Income was the only indicator of SES used in the current study.

In the New Zealand context, income disparity is evident between NZborn and born overseas (New Zealand Immigration Service, 2003). Immigrants earn on average $17.7 \%$ less than the native-born New Zealanders. Specifically, the census report points out that in terms of ethnicities of New Zealanders, "Other Ethnicities" and NZ Europeans have highest earnings leaving Asians with the lowest average income (NZIS, 2003).

The material and status conceptualizations of SES represent that individuals have better well-being if they are categorized in a higher SES band due to the monetary advantages as well as higher social status affiliations (Schnittker \& McLeod, 2005). Thus it is believed that higher income would be negatively related to perceptions of discrimination. Contrary to this belief, Gibbons et al. (2004) found a significant relationship between discrimination and SES wherein American Black adults who have higher SES experience more discrimination. They explained this as those American Black adults who 
were in the higher SES are likely to be employed and are more exposed to constant interaction with White counterparts.

Although individual studies in the discrimination literature reflects that ethnic discrimination is not related to SES (Combs et al., 2006; Lease, 2006; Phinney et al., 1993; Romero \& Roberts, 1998), the findings in Chapter 2 showed that SES is negatively related to perceptions of discrimination as reflected in the small but significant effect found in the meta-analytic study. In the studies included in the meta-analysis, SES was used either as a correlate of PD or as control variable. Hence, based on the meta-analysis' findings, the following hypothesis is proposed:

Hypothesis 7.5: Employees with lower income will perceive more discrimination in the workplace than employees with higher income.

\section{Distal Influences}

Aside from demographic differences, perceptions of discrimination are believed to be influenced by environmental factors. Gelfand's et al. (2005) Systems Model of Discrimination (SMD) refers to the internal systems of organizations that could serve as contexts of discrimination in the workplace. Thus, internal systems like formal and informal structures, organizational culture, strategy, human resource systems, leadership and organizational climate could serve as sources of employees' perceived discrimination. Based on SMD, these systems reflect discriminatory practices if the organization's decision makers or members of management are not sensitive to cultural changes brought about by increasing diversity in workplaces. Four organizational factors will be studied as antecedents of perceived 
discrimination namely justice perceptions, organizational practices, the organization's sector, and the size of the organization.

\section{Perceptions of organizational justice.}

The first organizational factor that could influence PD in the workplace is organizational justice perceptions. Gelfand's et al. (2005) internal systems of the organization can also serve as context for fairness perceptions and has led to substantial interest in organizational justice research. In the Systems Model of Discrimination (SMD) proposed by Gelfand and colleagues (2005), perceptions of fairness can be seen as reflections of a) the structures such as formal structures that are related to pay and promotion as well as informal structures that relate to workplace interpersonal relationships; and b) the human resource (HR) systems such as all facets of HR practices from selection to performance appraisal.

Justice in organizations has been a major research topic area in human resource management, industrial-organizational psychology and behaviour in workplaces (Cropanzano \& Greenberg, 1997). Organizational justice perceptions are currently measured through four dimension namely procedural, informational, interpersonal and distributive justice. Procedural justice is defined as perceiving that the process of distributing outcomes is fair. Informational justice is defined as the perceptions of timely and accurate communication of procedures. Interpersonal justice is the perceptions of being treated fairly and with respect by employers, while distributive justice is the perception of fair allocation of outcomes (Colquitt, 2001). Organizational justice has been extensively linked to positive organizational outcomes like satisfaction, commitment, and rule compliance among others. A quantitative 
integration of existing studies through a meta-analysis on this topic by Colquitt, Colon, Wesson, Porter, \& Ng (2001) showed that perceived justice in organizations predict job satisfaction, organizational commitment, withdrawal and negative reactions.

The common targets of discrimination in the workplace are the stigmatized individuals (Stone, Stone, \& Dipboye, 1992) due to their "stigmas" or marks (Goffman, 1963, in Stone-Romero, 2005) such as race, physical appearance, nationality and the like. Thus, as a consequence of stigmatization, targets are subjected to injustice in terms of getting lower outcomes than they deserve [distributive injustice], unfair allocation of resources [procedural injustice] and being treated less favourable in interactions [interactional injustice] (Stone-Romero \& Stone, 2004).

Justice perceptions have not been directly studied as a factor that could influence PD. Earlier studies attempted to link fairness perceptions with discrimination like the series of studies by Fernandez (1981, 1991, 1999). These studies revealed that different groups held different fairness perceptions like men felt being treated more fairly in organizations than women. Women and ethnic minority groups such as African Americans, Asian Americans, and Native Americans perceived that White men are the most favoured group showing a possible association between fairness and discrimination issues (Fernandez, 1981). More recent studies have attempted to link justice perceptions and PD as the criterion variable. For instance, Foley et al.'s (2002) study found that Hispanic lawyers' perceptions of discrimination are indirectly linked to distributive justice through the path of perceived glass ceiling. Specifically, they found that perceived ethnic discrimination is positively 
related to glass ceiling, and glass ceiling in turn was negatively related to perceptions of distributive justice. In a study focussing on gender discrimination, procedural justice and distributive justice wer found to be both negatively related to gender discrimination (Foley et al., 2005). In view of the direction of the relationship of justice perceptions with discrimination in previous studies, I propose the following hypothesis:

Hypothesis 7.6.1: Higher perceptions of procedural justice will be related to lower levels of PD in the workplace.

Hypothesis 7.6.2: Higher perceptions of interpersonal justice will be related to lower PD in the workplace.

Hypothesis 7.6.3: Higher perceptions of informational justice will be related to lower PD in the workplace.

Hypothesis 7.6.4: Higher perceptions of distributive justice will be related to lower PD in the workplace.

\section{Perceptions of organizational practices.}

The second factor that could influence PD is the perceptions about organizational practices. The measurement of organizational practices used in this study is rooted in the concept of organizational climate as a reflection of organizational culture. Organizational culture is another particular internal system that has been pointed out in the SMD (Gelfand, et al., 2005) as a context for discrimination in organizations. Based on the model, the underlying basic assumptions, values and cultures of the organization may propagate more subtle forms of discrimination. Further, the strategy being implemented by an organization can affect employee perceptions. Organizations with innovative strategies are more open to diversity in that 
structures give value to a diverse workforce (Cox et al., 1991; Dipboye \& Collela, 2005). However, employee perceptions of organizational culture has not been appropriately addressed as a means to eliminate or minimize discrimination in the workplace (Carnevale \& Stone, 1995; Triandis, Kuruwski, \& Gelfand, 1994) since most of the attention has been directed to more obvious organizational structures such as hiring policies (Gottfredson, 1992; Jackson; 1992).

Organizational culture emphasizes individual perceptions since its focus is how employees assign meanings on their work-related experiences and how these meanings affect their behaviours (Van Muijen, Koopman, De Witte, De Cock, Susanj, Lemoine et al., 1999). Positive perceptions regarding organizational culture has been linked to job satisfaction in various facets like satisfaction in pay, co-workers, supervisors, career advancement, with work itself, and with the job in general (Aarons \& Sawitsky, 2006; Goldston, 2008; Stebbins; 2008; Westerman \& Yamamura, 2007); turnover intentions (Aarons \& Sawitsky, 2006; Westerman \& Yamamura, 2007); organizational commitment (Sikorska-Simmons, 2005) and work-related stress (Hollifield, 2006).

Previous instruments used to measure organizational culture were found to be inadequate due to numerous dimensions that are usually not integrated (Baris, Ferreira, Assmar, Omar, Fischer, Huyhn et al., 2005) and were applied mostly in Western societies thus limiting the studies' generalizability (Fischer, Ferreira, Assmar, Redford, \& Harb, 2005). To address this problem, Baris and colleagues (2005) embarked on a six-country study to investigate further the dimensions of culture and to test a new 
instrument in both Western and non-Western settings. Data were collected from organizations in Argentina, Brazil, Malaysia, New Zealand, Turkey and the United States. The resulting factor structure of the scale, fully tested for its validity and reliability, has three dimensions, namely perceptions on employee orientation, formalization and innovation practices. Baris and colleagues (2005) define the three dimensions as follows: 1) Employee orientation practices pertain to perceptions that the organization's policies and practices are intended to support employees and to encourage interpersonal relationship; 2) Formalization practices relate to perceptions that the policies and practices are designed to impose control over employee behaviours; and 3) Innovation practices pertain to perceptions that organizations are courageous risk takers on issues related to decision-making and company growth.

Perceptions of discrimination as a direct outcome of organizational practices perceptions is practically a novel way of looking at antecedents of PD since to the best of my knowledge no studies have included these variables in one model. Given that organizational practices have been shown to be positively related to outcomes that are also (but negatively) affected by PD (e.g. job satisfaction, organizational commitment), I use these parallels for proposing the following hypothesis:

Hypothesis 7.7.1: Higher perceptions that organizations are employeeoriented will be related to lower perceptions of discrimination.

Hypothesis 7.7.2: More perceptions that organizations are less formal will be related to lower perceptions of discrimination. 
Hypothesis 7.7.3: More perceptions that organizations pursue a strategy of innovation will be related to lower perceptions of discrimination.

\section{Size and sector of organizations.}

The third and fourth factors that are considered as distal antecedents of PD are the size and sector of organizations. Organizational demographics are neglected in the literature of PD since the usual focus of studies is on individual characteristics. In this study, organizational characteristics, such as company sector and size will be explored as potential determinants of discrimination. The organizational sector may be source of PD due to differing practices in private compared to public sector companies. For instance, public organizations are regarded as more highly bureaucratic than private organizations due to the former's adherence to rigid and formalized policies and hierarchical structures (Baris et al., 2005).

Moreover, Arvey, Azevedo, Ostgaard and Raghuram (1996) view large organizations with formal human resource policies as having rigid employment policies and hierarchical structures compared to growing organizations that are more flexible since they have room for restructuring of their policies and manpower composition. Due to these centralized and fixed policies and non-flexible seniority-based orientation structures, large organizations are more susceptible to discrimination (Gelfand et al., 2005). Due to the similarity of the characteristics between large organizations and public organizations, it is expected that public organizations may be more susceptible to discrimination. Private organizations, on the other hand, have 
been found to be positively related to innovation strategies which is in turn regarded as desirable trait of an organization (Baris et al., 2005)

Historically, large organizations in the US and Western Europe in the 1960's-1970's have been classified as “monolithic" - organizations that are substantially homogenous (e.g. majority White male employees, with women and minorities in low status jobs). However, it is believed that since the 1990's most large organizations have undergone organizational change and have adapted the "Plural" and "Multicultural" models such that these organizations are more heterogeneous in demographics and more culturally inclusive in their policies (Cox, 1993). Organizations that attempted to be more culturally and demographically inclusive implemented of new policies such as affirmative action programs, equal opportunity rights laws, sexual harassment and compensation audits in order to reduce discrimination. However, more and more studies reveal that these new policies generate a new level of "reverse discrimination" or "positive discrimination" since the non-beneficiaries believe that these policies are discriminatory to them (Steeh \& Krysan, 1996). In the absence of a clear basis for the influence of size and sector of organizations in discrimination literature, a research question is posed instead of a hypothesis:

Research Question 7.4: Will employees from larger organizations perceive higher discrimination?

Research Question 7.5: Will employees from the public sector perceive more discrimination? 
In summary, this study aims to investigate the proximal and distal factors that are believed to influence PD. This study expects that proximal factors like age, ethnicity, birthplace, gender and income are related to PD. Moreover, distal factors like organizational justice perceptions and organizational practices perceptions are also expected to be related to PD. The study also aims to investigate the role of organizational size and sector as distal factors. Lastly, the study explores the influence of the 2-way interaction of gender and ethnicity as well the 3-way interactions of ethnicity-genderbirthplace on PD. The variables employed in this study as antecedents of PD in the workplace have not been simultaneously investigated in one model. Thus, the systematic investigation of these variables in a combined model and in a large sample is deemed important as a new contribution to the discrimination literature, especially in the workplace context.

\section{Method}

\section{Participants}

\section{Total Sample}

The total sample was composed of 540 employees (females 50\%) working in 24 organizations in the Wellington Region of New Zealand (NZ). Participants' ages ranged from 16 to 65 years, with an average of 34.8 years $(S D=14.5)$. The gender distribution and mean age are comparable to the recent census of the national population conducted by Statistics New Zealand (2006). Most of the respondents are occupied the position categories/levels of professionals $(25.0 \%)$, middle managers/supervisors $(20.4 \%)$, clerical and administrative workers $(14.8 \%)$ and sales workers (11.1\%). Moreover, the 
majority of the respondents had permanent and full-time jobs (86.7\%). Their tenures in their respective organizations ranged from half a year to 45 years, with a mean of 5.0 years $(S D=6.0)$. Table 7.1 presents the participants personal demographics. The participants were asked to indicate their country of birth. Their responses were categorized as either New Zealand-born or Immigrants (born outside New Zealand or first generation immigrants). Seventeen participants did not respond whether they were born in New Zealand or not, thus leaving a total of 168 for the Immigrant sample and 355 for NZ-born sample. These seventeen participants were excluded in the following analysis.

Table 7.1

Employee Participants' Personal Demographics

\begin{tabular}{lccc}
\hline & Total & Immigrants & NZ-born \\
\hline & & & \\
No. of Participants & 540 & 168 & 355 \\
Gender & & & \\
Male (\%) & $259(48 \%)$ & $78(46.4 \%)$ & $176(49.6 \%)$ \\
Female (\%) & $270(50 \%)$ & $89(53 \%)$ & $176(49.6 \%)$ \\
Not Stated (\%) & $11(2 \%)$ & $1(0.6 \%)$ & $3(0.8 \%)$ \\
$\quad$ & & \\
Age - Mean (SD) & $34.8(14.6)$ & $36.4(13.9)$ & $34.1(14.8)$ \\
Tenure in the Organization (years) & & & \\
$\quad$ - Mean (SD) & $5.0(6.0)$ & $4.5(5.5)$ & $5.3(6.4)$ \\
Job Status & & & \\
$\quad$ - Permanent/Full-time & $86.7 \%$ & $89.3 \%$ & $87.0 \%$ \\
$\quad$ - Fixed Term/Contract & $8.3 \%$ & $5.4 \%$ & $9.6 \%$ \\
$\quad$ - Others & $2.8 \%$ & $4.8 \%$ & $2.0 \%$ \\
$\quad$ - Not stated & $2.2 \%$ & $.6 \%$ & $1.4 \%$ \\
Job Levels & & & \\
$\quad$ - Chief Executive/General Managers & $1.1 \%$ & $0.6 \%$ & $1.4 \%$ \\
$\quad$ - Middle Managers/Supervisors & $20.4 \%$ & $21.4 \%$ & $20.8 \%$ \\
$\quad$ - Professionals & $25.0 \%$ & $27.4 \%$ & $23.9 \%$ \\
$\quad$ - Technicians/Trade Workers & $4.1 \%$ & $6.0 \%$ & $3.4 \%$ \\
- Community/Personal Services Worker & $2.0 \%$ & $1.2 \%$ & $2.5 \%$ \\
$\quad$ - Clerical/Administrative Worker & $14.8 \%$ & $11.3 \%$ & $16.9 \%$ \\
- Sales Worker & $11.1 \%$ & $14.3 \%$ & $9.0 \%$ \\
- Labourer & $6.5 \%$ & $5.4 \%$ & $7.0 \%$ \\
- Others & $7.4 \%$ & $5.4 \%$ & $8.2 \%$ \\
- Not Stated & $7.6 \%$ & $6.6 \%$ & $6.7 \%$ \\
\hline$\quad$ & & &
\end{tabular}




\section{Immigrants' Sample.}

One hundred sixty eight $(31.11 \%)$ of the total 540 respondents were born outside of NZ. This percentage is a bit higher than what is indicated in the recent census wherein overseas born people in NZ comprise around $23 \%$ of the population (Statistics New Zealand, 2006). The immigrants' ages have a mean age of 36.4 years $(S D=13.9)$ Most of the respondents occupied the position categories/levels of professionals (27.4\%), middle managers/supervisors $(21.4 \%)$ and sales workers $(14.3 \%)$. The majority of the respondents had permanent and full-time jobs (89.3\%). Their tenures in their respective organizations had a mean of 4.5 years $(S D=5.5)$. More than half of the participants $(53.0 \%)$ worked in large organizations. Almost a quarter of the immigrant sample worked in Retail Trade (23.8\%), followed by Education and Training (17.3\%) and Information Media and Telecommunications (14.9\%). The immigrants' personal and organizational demographics are also presented in Table 7.1. A total of 42 countries were reported by the Immigrant respondents as their country of birth. Thirteen or $7.74 \%$ of the immigrant sample did not indicate their country of birth. The immigrant sample's birthplaces are consistent with the recent population census wherein it is noted that the most common birthplace is UK followed by countries in Asia (Statistics New Zealand, 2006). Please refer to Table 7.2 for the breakdown of the immigrant sample's countries of birth.

\section{New Zealand-born Sample.}

New Zealand-born participants comprise the majority of the total sample (355 or $66 \%$ of the total sample). The mean age for this group is 34.1 years $(S D=14.8)$. The top three position categories being occupied by the NZ-born 
Table 7.2

Immigrants' Countries of Birth

\begin{tabular}{|c|c|c|c|c|}
\hline & & & Subtotal & $\%$ \\
\hline \multicolumn{3}{|c|}{ European-Descent } & 67 & $39.88 \%$ \\
\hline & Andorra & 1 & & \\
\hline & Australia & 12 & & \\
\hline & Canada & 3 & & \\
\hline & France & 1 & & \\
\hline & Germany & 2 & & \\
\hline & Ireland & 1 & & \\
\hline & Italy & 1 & & \\
\hline & Netherlands & 2 & & \\
\hline & Norway & 1 & & \\
\hline & Romania & 1 & & \\
\hline & Russia & 1 & & \\
\hline & United Kingdom & 38 & & \\
\hline & United States of America & 3 & & \\
\hline \multirow{12}{*}{ Asian } & & & 53 & $31.55 \%$ \\
\hline & China & 12 & & \\
\hline & India & 12 & & \\
\hline & Indonesia & 2 & & \\
\hline & Korea & 3 & & \\
\hline & Malaysia & 8 & & \\
\hline & Mongolia & 1 & & \\
\hline & Philippines & 7 & & \\
\hline & Singapore & 2 & & \\
\hline & Sri Lanka & 4 & & \\
\hline & Taiwan & 1 & & \\
\hline & Vietnam & 1 & & \\
\hline \multirow[t]{19}{*}{ Other } & & & 35 & $20.83 \%$ \\
\hline & Barbados & 1 & & \\
\hline & British Virgin Islands & 2 & & \\
\hline & Cook Islands & 1 & & \\
\hline & Eritrea & 1 & & \\
\hline & Fiji & 10 & & \\
\hline & Iraq & 1 & & \\
\hline & Kenya & 1 & & \\
\hline & Niue & 1 & & \\
\hline & Pakistan & 2 & & \\
\hline & Samoa & 5 & & \\
\hline & Solomon Islands & 1 & & \\
\hline & South Africa & 2 & & \\
\hline & Tokelau & 1 & & \\
\hline & Tonga & 1 & & \\
\hline & Turkey & 1 & & \\
\hline & Tuvalu & 1 & & \\
\hline & Western Sahara & 1 & & \\
\hline & Zimbabwe & 2 & & \\
\hline \multicolumn{3}{|c|}{ Did Not State Country of Birth } & 13 & $7.74 \%$ \\
\hline & & Total & 168 & $100 \%$ \\
\hline
\end{tabular}


are professionals $(23.9 \%)$, middle managers/supervisors $(20.8 \%)$ and clerical/administrative workers $(16.9 \%)$. The majority of the respondents had permanent and full-time jobs $(87.0 \%)$. Their mean tenure in their respective organizations was 5.3 years $(S D=5.4)$. The respondents in this group worked mostly in large $(44.2 \%)$ or medium $(40.8 \%)$ organizations. Similar to the immigrant groups almost a quarter of the immigrant sample work in Retail Trade (22.8\%), followed by Education and Training (19.4\%). However, the third highest industry is Financial and Insurance Services (11.5\%) rather than the Information Media and Telecommunications. See NZ-born group's personal demographics in Table 7.1 in the previous page.

The NZ-born participants indicated various ethnicities such European (58.3\%), New Zealander (5.4\%), Kiwi (13.5\%), Maori (9.6\%), Pacific Islanders (3.4\%), Asian (3.7\%) and Others (2.0\%). The choices of responses for ethnicity were patterned from the NZ Statistics Survey (2006). The NZ census permits the reporting of multiple ethnic background (NZ Statistics, 2001, 2006). Thus, I could not combine the "New Zealander" and "Kiwi" with the European background because any NZ-born nationals could indicate that they are Kiwi or New Zealander regardless of their ethnic background. Thus, these two terms are rather loosely used to indicate their nationality (Spoonley, 1993) unlike "New Zealand Pakeha" which has historically been referred to New Zealanders with European ancestry. In fact, it has been pointed out by Callister (2004) that New Zealand European has an alternate label of "Pakeha" in NZ statistical surveys. Further, those who were categorized as "Maori" have either indicated pure Maori ancestry or mixed Maori ancestry. Lastly, there were participants who did not state any ethnic background (4.2\%). See 
complete list of ethnic backgrounds of New Zealand-born participants in Table

7.3 .

Table 7.3

Ethnic Backgrounds of New Zealand-Born Participants

\begin{tabular}{|c|c|c|c|}
\hline & & Subtotal & $\%$ \\
\hline European & & 207 & $58.3 \%$ \\
\hline Dutch & 2 & & \\
\hline European nfd & 44 & & \\
\hline New Zealand European & 117 & & \\
\hline New Zealand Pakeha & 44 & & \\
\hline New Zealander & & 19 & $5.4 \%$ \\
\hline Kiwi & & 48 & $13.5 \%$ \\
\hline Maori & & 34 & $9.6 \%$ \\
\hline Maori & 13 & & \\
\hline Maori/Chinese & 1 & & \\
\hline New Zealand Maori & 15 & & \\
\hline NZ Maori/Pakeha & 2 & & \\
\hline Maori/Niuean/European & 1 & & \\
\hline Maori/Samoan/European & 1 & & \\
\hline Maori/Samoan/Finnish & 1 & & \\
\hline Pacific Peoples & & 12 & $3.4 \%$ \\
\hline Pacific Peoples nfd & 1 & & \\
\hline Cook Island & 1 & & \\
\hline Niuean & 1 & & \\
\hline Polynesian & 1 & & \\
\hline Samoan & 7 & & \\
\hline Tokelauan & 1 & & \\
\hline Asian & & 13 & $3.7 \%$ \\
\hline Asian nfd & 2 & & \\
\hline Chinese & 3 & & \\
\hline Indian & 6 & & \\
\hline Euroasian & 1 & & \\
\hline NZ Filipino & 1 & & \\
\hline Others & & 7 & $2.0 \%$ \\
\hline \multirow[t]{2}{*}{ Did Not State Ethnic Background } & & 15 & $4.2 \%$ \\
\hline & Total & 355 & $100 \%$ \\
\hline
\end{tabular}




\section{Organizations.}

Participants came from 24 organizations in the Wellington Region. The organization's size was categorized into small (30 employees or less), medium (30-100 employees) and large (over 100 employees). More than half (62.5\%) of the participating organizations had more than 100 employees. Moreover, organizations were also categorized into either private or public sector. Private organizations dominated the sample $(58 \%)$. The listing of the organizational demographics is found in Table 7.4.

Table 7.4

Organization's Demographics

\begin{tabular}{lcc}
\hline & No. & $\%$ \\
\hline $\begin{array}{l}\text { Sector of the Organization } \\
\text { - Private }\end{array}$ & \\
- Public & 14 & $58.0 \%$ \\
Total & 10 & $42.0 \%$ \\
Size of the Organization & 24 & $100 \%$ \\
- Small & & \\
- Medium & 3 & $12.5 . \%$ \\
- Large & 6 & $25.0 \%$ \\
Total & 15 & $62.5 .0 \%$ \\
& 24 & $100.0 \%$ \\
\hline
\end{tabular}

\section{Measures}

\section{Perceptions of Discrimination}

The Employee Perceptions of Discrimination in the Workplace Scales (EPDWS) is a modified version of the original Immigrants' PD in the 
Workplace Scale (IPDWS-Pilot) used in Study 3. Some items in the IPDWSPilot were rephrased in the EPDWS to make them applicable to both immigrants and the NZ-born. Participants were requested to respond to a total of 34 items that were designed to measure employee PD on different workplace issues (see Appendix D). The three scales were similar to IPDWSPilot namely Finding Work (14 items), Work Conditions (14 items) and Work Relationships (6 items). Finding work pertained to perceptions of discrimination in relation to how people are hired in their organizations in accordance to certain practices or company regulations (e.g. "Having an accent is a barrier to obtaining employment in this organization"). Respondents indicated their response on a scale of 1 (strongly disagree) to 5 (strongly agree) with high scores indicating higher perceptions of discrimination.

The second scale was Working Conditions containing items that measure perceived discriminatory practices or policies affecting employees in terms of compensation, benefits, training and career progression (e.g. "There is a clearly visible career line of increasing rewards and promotion for me in this organization"). Similar to finding work, the participants indicated their response on a scale of 1 (strongly disagree) to 5 (strongly agree) with high scores indicating higher PD.

The third scale was Work Relationships that pertained to discriminatory perceptions for the quality of interactions and relationships with co-workers and superiors within the working environment (e.g. "At work, my co-workers make joke or negative comments about people of my 
cultural/ethnic background). The responses were on a scale from 1 (Never) to 5 (All the time) with high scores indicating higher PD.

A separate factor analysis was conducted on the EPDWS. Principal component analysis (PCA) using oblique rotation (Direct Oblimin with Kaiser normalization) was used to determine the factor structure of each of three scales of the EPDWS. Oblique rotation was used due to the assumption that the components are intercorrelated. Similar to Study 3-A, a number of guidelines were observed to determine the number of factors to be extracted from the principal component analysis using oblique rotation. The guidelines were: 1) Kaiser's (1970) greater-than-one rule; 3) inspection of the Scree Plot from the Principal Component Analysis; 4) retention of items with loadings of .50 or better; and 5) deletion of items that are cross-loaded onto two or more factors showing a difference of .10 or less between the loadings.

\section{Finding work.}

The initial extraction resulted in 4 eigenvalues greater than 1 (3.19, $2.17,1.39,1.06)$. However, inspection of the scree plot and the pattern matrix suggested three interpretable factors accounting for $22.82 \%$ of the total variance. Based on item content, the three factors were named non-recognition of education and experience (6 items), networking (3 items) and non-local (3 items). Initially, there were four items in non-local. Deleting one item (item D14) improved the reliability coefficient from $\alpha=.52$ to $\alpha=.55$.

The first factor (non-recognition of education and experience) pertains to the perception that authorities do not acknowledge the education and work experience obtained either within New Zealand or outside of New Zealand in the case of immigrants (e.g. "My educational qualifications are recognized in 
my job" - recoded as a negatively worded item). The items for nonrecognition of education and experience loaded from .61 to .82 (mean loading $=.72$ ). The second factor, networking, describes the importance of having contact persons in organization in order to be considered for a job in that organization (e.g. "It is easy to get a job if you know people in this organization"). The items for networking loaded from .73 to .82 (mean loading $=.77$ ). Lastly, the third factor (non-local) pertains to the individual's perceptions of his/her personal characteristics as the basis for the perceptions of discrimination (e.g. "Having an accent is a barrier to obtaining employment in this organization). Non-local items loaded from .53 to .71 (mean loading $=$ .63). The factors networking and non-local were not included in the succeeding analysis since they do not represent personal experience; rather they reflect general perceptions of the system. See Table 7.5 for the 12 items of the scale Finding Work with the pattern matrix's factor loadings, eigenvalues, variances and reliability coefficients.

\section{Work conditions.}

Initial extraction generated five eigenvalues greater than $1(3.54,1.55$, $1.25,1.18$ and 1.04). Inspection of the scree plot and the pattern matrix also suggested 1-2 interpretable factors accounting for $25.25 \%$ of the total variance. Thus, a two-factor solution was again subjected to PCA. Upon inspection of the factor loadings in the pattern matrix, two items were deleted since the factor loading did not meet the .5 criteria. Initially, a two-factor structure was considered but in the end it was decided to retain just one factor since the second factor's internal reliability was only $\alpha=.30$. Deletion of any of the 4 items in the second factor did not improve the reliability coefficient. 
Table 7.5

Factor Loadings, Variances and Reliability Coefficients for Finding Work

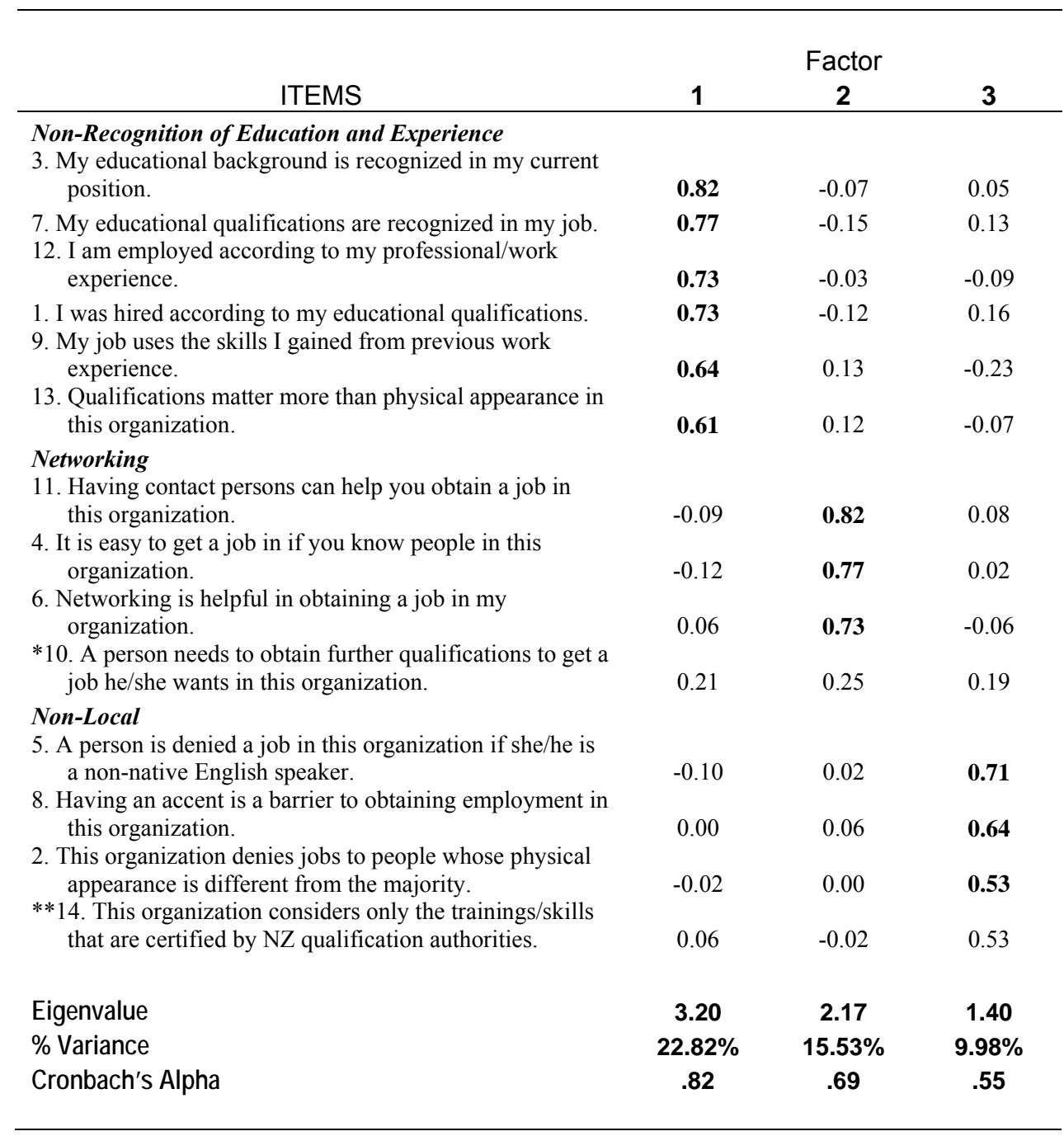

Note: items included in the factor are written in boldface; * item deleted due to low factor loadings; **item deleted to increase internal reliability

Thus, a single factor structure was retained and based on item content it was labelled as "career path" (4 items). Hindered Career Path refers to discriminatory perceptions that hinder the professional or career advancement of the individual (e.g. "I have a good chance for promotion" - recoded as a negatively worded item). The hindered career path items 
loaded from .66 to .85 (mean loading $=.76$ ). The overall factor loadings, eigenvalues, variances and reliability coefficients are reported in Table 7.6.

Table 7.6

Factor Loadings, Variances and Reliability Coefficients for Work Conditions

\begin{tabular}{|c|c|c|}
\hline \multirow[b]{2}{*}{ ITEMS } & \multicolumn{2}{|c|}{ Factor } \\
\hline & 1 & 2 \\
\hline $\begin{array}{l}\text { Hindered Career Path } \\
\text { 28. There is a clearly visible career line of increasing rewards and } \\
\text { promotion for me in this organization. }\end{array}$ & 0.81 & 0.07 \\
\hline 15. I have opportunities for advancement. & 0.79 & 0.00 \\
\hline 21. I have a good chance for promotion. & 0.77 & 0.06 \\
\hline 25. My promotion at this organization has been regular. & 0.60 & 0.05 \\
\hline $\begin{array}{l}\text { **16. I feel my salary/wage is fair considering what other people in } \\
\text { this firm are paid. }\end{array}$ & 0.58 & -0.03 \\
\hline $\begin{array}{l}* * 18 \text {. If feel my salary/wage is fair considering what other firms in } \\
\text { this sector pay. }\end{array}$ & 0.56 & 0.05 \\
\hline $\begin{array}{l}\text { *26. This organization thinks about the welfare of every employee } \\
\text { regardless of their ethnic background. }\end{array}$ & 0.41 & -0.33 \\
\hline $\begin{array}{l}* 20 . \text { This organisation's policies and procedures are fair to all } \\
\text { employees regardless of their ethnic background. }\end{array}$ & 0.38 & -0.28 \\
\hline $\begin{array}{l}\text { 19. Fringe benefits (e.g. leave entitlements, insurance) in this } \\
\text { organization are not commensurate to the employees' line of } \\
\text { work. }\end{array}$ & 0.22 & 0.71 \\
\hline $\begin{array}{l}\text { 17. Policies and procedures of this organization are applicable only } \\
\text { to local New Zealanders. }\end{array}$ & 0.23 & 0.68 \\
\hline *22. I am in a dead-end job. & -0.27 & 0.51 \\
\hline $\begin{array}{l}\text { *24. My salary/wage is not commensurate to the amount of work I } \\
\text { do in this organization. }\end{array}$ & -0.12 & 0.43 \\
\hline $\begin{array}{l}\text { *27. Paid sick leave entitlement given by this organization is } \\
\text { comparable to other similar sectors. }\end{array}$ & 0.10 & -0.20 \\
\hline $\begin{array}{l}\text { *23. I get health insurance and other benefits comparable to other } \\
\text { firms in this sector. }\end{array}$ & -0.01 & -0.15 \\
\hline Eigenvalue & 3.54 & 1.55 \\
\hline$\%$ Variance & $25.25 \%$ & $11.05 \%$ \\
\hline Cronbach's Alpha & .81 & .30 \\
\hline
\end{tabular}

Note: items included in the factor are written in boldface; * item deleted due to low factor loadings; ** item deleted due to factorial incongruence

\section{Work relationships.}

Two initial eigenvalues greater than 1 were found (eigenvalues of 2.89 and 1.27). Inspection of the scree plot and the pattern matrix suggested 1 or 2 interpretable factors accounting together for $48.17 \%$ of 
the total variance. Thus, a two-factor structure was considered and labelled as disharmonious work dynamics (4 items) and derogation (2 items). The first factor (disharmonious work dynamics) pertains to perceptions that the quality of work-related interactions is dependent on group membership (e.g. "I have excellent working relationships with supervisors regardless of my ethnic background" - recoded as negatively worded items). This factor has item loadings from .64 to .88 (mean loading $=.79$ ). The last factor (derogation) contains perceptions that people make fun of those who belong to other ethnic group (e.g. "At work, my supervisor makes jokes or negative comments about people of my cultural/ethnic background"). Presented in Table 7.7 are the 6 items, with loadings, eigenvalues, variances and reliability coefficients.

Table 7.7

Factor Loadings, Variances and Reliability Coefficients for Work Relationships

\begin{tabular}{lcc}
\hline \multicolumn{1}{c}{ ITEMS } & \multicolumn{2}{c}{ Factor } \\
& $\mathbf{1}$ & $\mathbf{2}$ \\
\hline & & \\
& & \\
$\begin{array}{l}\text { Disharmonious Work Dynamics: } \\
\text { 32. I have excellent working relationships with supervisors } \\
\quad \text { regardless of my cultural/ethnic background. }\end{array}$ & $\mathbf{0 . 8 8}$ & 0.13 \\
$\begin{array}{l}\text { 31. My contributions at work are recognized by my } \\
\text { supervisor regardless of my cultural/ethnic background. }\end{array}$ & $\mathbf{0 . 8 2}$ & 0.06 \\
$\begin{array}{l}\text { 33. My contributions at work are recognized by my co- } \\
\text { workers regardless of cultural/ethnic background. }\end{array}$ & $\mathbf{0 . 8 1}$ & -0.10 \\
$\begin{array}{l}\text { 29. I have excellent working relationships with co-workers } \\
\text { regardless of my cultural/ethnic background. }\end{array}$ & $\mathbf{0 . 6 4}$ & -0.21 \\
$\begin{array}{l}\text { Derogation: } \\
\text { 30. At work, my supervisor makes jokes or negative } \\
\quad \text { comments about people of my cultural/ethnic background. }\end{array}$ & -0.01 & $\mathbf{0 . 8 8}$ \\
$\begin{array}{l}\text { 34. At work, my co-workers make joke or negative comments } \\
\text { about people of my cultural/ethnic background. }\end{array}$ & 0.00 & $\mathbf{0 . 8 8}$ \\
$\begin{array}{l}\text { Eigenvalue } \\
\text { \% Variance } \\
\text { Cronbach's Alpha }\end{array}$ & $\mathbf{2 . 8 9}$ & $\mathbf{1 . 2 7}$ \\
\hline
\end{tabular}

Note: items included in the factor are written in boldface 


\section{Structural equivalence.}

A test for equivalence was conducted on the EPDWS's factor solution using Procrustean Target Rotation technique. This technique is used to compare the rotated factor matrices and factorial agreement coefficients of the immigrants' responses with that of the NZ-born responses to ensure that the items have the same meanings for both groups. The rotated factor solution for NZ-born was used as the norm and the immigrants' group rotated factor solution as the target group. It is suggested that factorial agreement coefficients higher than .95 signify equivalence, while values less than 0.90 (van de Vijver \& Leung, 1997) or 0.85 (Ten Berge, 1986) indicate factorial incongruence. In this study, the resulting values for the factorial agreement coefficients such as the Tucker's phi (proportionality coefficient) are all above .91. It is therefore valid to assume that the items in the EPDWS scale have the same meaning for both groups, thereby allowing us to interpret the mean differences of the whole sample accordingly.

\section{Internal consistency.}

The internal consistency values (Cronbach's alphas) of the PD factors retained in the succeeding analysis met the minimum acceptable alpha coefficient levels of 0.70 suggested by Nunnally and Bernstein (1994). Thus, four PD factors were used for analysis namely NonRecognition of Education \& Experience, Hindered Career Path, Disharmonious Work Dynamics and Derogation. Reliability coefficients of each factor are included in the tables illustrating the factor structures of each scale. 


\section{Personal and Work-Related Demographics}

Various questions pertaining to personal and job-related information were asked of the respondents. Among the demographic information obtained, age, gender, ethnicity, country of birth and income will be used in the analysis together with organizational demographics such as organizational sector and size. Similar to Study 3, choice or responses (categories/subcategories) were patterned from the NZ Statistics Survey (2006) and the standard classification as prescribed by the Australian and New Zealand Standard Industrial Classification (ANZSIC) 2006

\section{Organizational Practices and Decision-Making (OP)}

Employee perceptions were measured through a 15-item scale measuring three dimensions namely employee orientation, formalization and innovation practices. Responses were on a 7 -point scale $(1=$ Never to 7=Always). The items were either newly constructed or adapted by Baris et al. (2005), primarily based on work by Quinn and Spreitzer (1991) and van Muijen et al. (1999). The first dimension, innovation, refers to orientation towards innovation, risk taking and performance orientation. Higher scores are considered more desirable. The second dimension is formalization which measure perceptions of organizational practices related to a highly regulated, formal work atmosphere. Higher score are often considered as reducing productivity and innovation. The third and last dimension is employee orientation or support which is composed of statements related to support for employees and/or orientation towards 
employees. Higher scores for employee orientation are associated with more productive and healthy work environments. Cronbach's alphas for the organizational practices dimensions are .84 for employee orientation, .85 for formalization, and .78 for innovation.

\section{Organizational Justice Perceptions (OJP)}

Perceptions of justice in organizations were measured through a 20-item scale by Colquitt (2001) indicating responses to four dimensions namely procedural justice (7 items), interpersonal justice (4 items), informational justice (5 items) and distributive justice (4 items). Responses were on a 7 -point scale $(1=$ Not at all to $7=$ To a great extent $)$ with higher scores reflecting more perceived justice. Sample items for each dimension are a) procedural justice - "Have those procedures been free of bias?"; b) interpersonal justice - "Has s/he treated you in a polite manner?"; c) informational justice - "Has s/he communicated details in a timely manner?"; and d) distributive justice - "Is the outcome of these decisions appropriate for the work you have completed?". Cronbach's alphas for procedural, interpersonal, informational and distributive justice are $.87, .87, .91$ and .89 respectively.

\section{Procedure}

Participants were recruited by third-year university students enrolled in an Industrial/Organizational course as part of a class assignment during the first trimester of 2007. The students were instructed to form 25 groups with 3 members each. Each group was asked to identify an organization (through personal contacts, e.g. friends, parents, 
neighbours) and collect a minimum number of 20 surveys from employees working in that organization. Students who were employed were reminded not to collect surveys from an organization in which they are currently employed (to avoid conflicts of interests, e.g., obtaining reports from any potential subordinates for which the student might have responsibility over). The students were further instructed to collect a proportionate number of New Zealand-born employees and immigrant employees (first generation immigrants or those born outside of New Zealand).

The participants were asked whether they would be interested in participating in a study on work perceptions. They were further informed that participation is voluntary and they will receive a small token (chocolate bar). The questionnaires were filled out by the participants in their most convenient time and place. The completed survey were placed in an unmarked envelope and sealed to preserve anonymity of respondents within the organization before handing it back to the students or contact persons who were collecting the data.

The exact response rate could not be determined due to the method of data collection used in this study. Since the instruction for each group to submit a minimum of 20 answered questionnaires, the students were initially given 20 blank questionnaires per group. However, they were allowed to photocopy the blank questionnaires if they though they would need more. Hence, the final number of questionnaires given out was undeterminable. Data was coded into spreadsheets and all analyses were done using the SPSS statistical package. 


\section{Results}

\section{Descriptives}

The item means and standard deviations of all PD factors based on participant characteristics are found in Table 7.8. Further, the descriptive statistics for the scales used as distal antecedents are shown in Table 7.9.

\section{Hierarchical Regression Analyses}

Separate hierarchical regression analyses were computed to test the hypothesis for each PD factor as an outcome variable. At all times, the proximal antecedents like age, gender $($ coded as male $=0$, female $=1)$, ethnicity (coded as White European $=0$, Non-White $=1)$, birthplace (coded as NZ-born $=0$, overseas born $=1$ ) and income were entered at first step. At Step 2, 2-way and 3-way interactions of some proximal antecedents were tested for their incremental validity, including the 2-way interaction of ethnicity and birthplace; ethnicity and gender; birthplace and gender; and the 3-way interaction of ethnicity, birthplace and gender. Lastly, all distal antecedents such as organizational sector (coded as $0=$ private, $1=$ public), organizational size (treated as continuous variable like income), organizational justice perceptions and organizational practices perceptions were entered at Step 3 of each regression equation to determine their incremental validity. The following are the results of the four sets of regression analyses: 
Table 7.8

Descriptive Statistics for the PD Factors and Participant Demographics

\begin{tabular}{|c|c|c|c|c|c|c|c|c|c|c|c|c|}
\hline & \multicolumn{3}{|c|}{$\begin{array}{l}\text { Non-Recognition of Educ } \\
\text { \& Experience }\end{array}$} & \multicolumn{3}{|c|}{ Hindered Career Path } & \multicolumn{3}{|c|}{$\begin{array}{c}\text { Disharmonious Work } \\
\text { Dynamics }\end{array}$} & \multicolumn{3}{|c|}{ Derogation } \\
\hline & $\mathbf{N}$ & $M$ & $S D$ & $\mathbf{N}$ & $M$ & $S D$ & $\mathbf{N}$ & $M$ & $S D$ & $\mathbf{N}$ & $M$ & $S D$ \\
\hline \multicolumn{13}{|l|}{ Ethnicity } \\
\hline European White & 279 & 2.62 & .84 & 280 & 2.89 & .94 & 279 & 1.81 & .74 & 279 & 1.68 & .90 \\
\hline Non-White & 133 & 2.62 & .95 & 133 & 2.67 & .90 & 133 & 1.82 & .71 & 133 & 1.94 & 1.05 \\
\hline Total & 412 & 2.62 & .87 & 413 & 2.81 & .93 & 412 & 1.82 & .73 & 412 & 1.76 & .96 \\
\hline \multicolumn{13}{|l|}{ Birthplace } \\
\hline NZ-born & 353 & 2.72 & .84 & 355 & 2.85 & .87 & 355 & 1.86 & .72 & 354 & 1.68 & .88 \\
\hline Immigrants & 166 & 2.49 & .92 & 167 & 2.83 & 1.00 & 166 & 1.84 & .76 & 167 & 1.96 & 1.05 \\
\hline Total & 519 & 2.65 & .87 & 522 & 2.85 & .92 & 521 & 1.86 & .73 & 521 & 1.77 & .95 \\
\hline \multicolumn{13}{|l|}{ Gender } \\
\hline Male & 252 & 2.63 & .84 & 253 & 2.79 & .88 & 253 & 1.89 & .75 & 253 & 1.79 & .91 \\
\hline Female & 263 & 2.64 & .89 & 265 & 2.90 & .95 & 264 & 1.83 & .72 & 264 & 1.77 & .99 \\
\hline Total & 515 & 2.64 & .87 & 518 & 2.84 & .92 & 517 & 1.86 & .73 & 517 & 1.78 & .95 \\
\hline
\end{tabular}


Table 7.9

Psychometric Properties of PD Distal Antecedents

\begin{tabular}{lcccc}
\hline & & $\begin{array}{c}\text { Range of } \\
\text { Responses }\end{array}$ & $M$ & $S D$ \\
\hline Organizational Practices & $\mathrm{N}$ & & & \\
& & & & \\
Employee Orientation & 521 & $1-7$ & 4.90 & 1.10 \\
Formalization & 521 & $1-7$ & 4.86 & 1.15 \\
Innovation & 521 & $1-7$ & 4.51 & 1.06 \\
& & & & \\
Justice Perceptions & & & & \\
Procedural Justice & 521 & $1-7$ & 5.19 & 1.24 \\
Interpersonal Justice & 522 & $1-7$ & 5.68 & 1.23 \\
Informational Justice & 522 & $1-7$ & 4.56 & 1.13 \\
Distributive Justice & 518 & $1-7$ & 4.56 & 1.23 \\
& & & & \\
\hline
\end{tabular}

\section{Non-recognition of education \& experience.}

The overall regression model predicting Non-recognition of education \& experience was significant: $F(18,375)=8.44, \mathrm{p}<.001$, and accounted for $28.8 \%$ of the total variance. The entry of proximal factors in step 1 resulted in a significant incremental change in $R^{2}$ $\left(\Delta R^{2}=.22, \Delta F(5,388)=22.02, p<.001\right)$. Age and income were significant predictors of lower PD related to education and experience across the three steps. The findings for age rejected Hypothesis 7.4 (H7.4), which predicted that increasing age would be associated with higher PD. Older employees reported less discrimination in this study. The hypothesis on income (H7.5) was however supported. Thus, employees with higher income perceived less discrimination. Further, the addition of the 2-way and 3-way interactions in Step 2 did not result in a statistically significant $\Delta R^{2}$. In the final step, the addition of distal antecedents accounted for $6 \%$ of the variance: $\Delta \mathrm{F}(9,375)=3.58, \mathrm{p}<.001$. However, examination of 
the beta values showed that only informational justice perceptions (supporting H7.6.3) and organizational size (Research Question [RQ] 7.4) were significant distal predictors of PD. This means that greater informational justice reduces discrimination, and that employees in larger organizations report less discrimination. Thus, only age, income, informational justice and size of the organization were predictors of REE. Results are shown in Table 7.10.

Table 7.10

Standardized Coefficients ( $\beta$ ) in Hierarchical Regressions for the Prediction of Job-Entry Perceptions of Discrimination

\begin{tabular}{|c|c|c|c|}
\hline \multirow[b]{2}{*}{ Predictors } & \multicolumn{3}{|c|}{$\begin{array}{c}\text { Non-Recognition of Educ \& Experience } \\
\text { Step }\end{array}$} \\
\hline & 1 & 2 & 3 \\
\hline \multicolumn{4}{|l|}{ Proximal Influences } \\
\hline Age & $-.20 * \star \star$ & $-.20^{\star * *}$ & $-.19 * \star \star$ \\
\hline Gender & -.05 & -.06 & -.07 \\
\hline White European (WE) & -.09 & -.05 & -.12 \\
\hline Birthplace & -.08 & -.03 & -.10 \\
\hline Income & $-.36^{\star \star \star}$ & $-.36^{\star \star *}$ & $-.35^{\star \star *}$ \\
\hline \multicolumn{4}{|l|}{ 2-way/3-way Interactions } \\
\hline WE x Birthplace & & -.07 & .01 \\
\hline WE x Gender & & .04 & .06 \\
\hline Birthplace x Gender & & .03 & .09 \\
\hline WE x Birthplace x Gender & & -.08 & -.15 \\
\hline \multicolumn{4}{|l|}{ Distal Influences } \\
\hline JP - Procedural & & & -.03 \\
\hline JP - Interpersonal & & & -.06 \\
\hline JP - Informational & & & $-.13^{*}$ \\
\hline JP - Distributive & & & -.06 \\
\hline OP - Employee Orientation & & & .00 \\
\hline OP - Formalization & & & -.05 \\
\hline OP - Innovation & & & .10 \\
\hline Sector & & & -.07 \\
\hline Size & & & $-.10^{*}$ \\
\hline$R^{2}$ & .22 & .23 & .29 \\
\hline$\Delta R^{2}$ & $.22^{\star \star \star}$ & .01 & $.06^{\star \star \star}$ \\
\hline
\end{tabular}

Note: Educ = Education; JP = Justice Perceptions; OP = Organizational Practices; Gender $(0=$ Male, $1=$ Female $) ; W E(0=$ White European-descent, $1=$ Non-WE); Birthplace $(0=$ NZ, $1=$ Overseas); Sector $(0=$ private, $1=$ public $)$; * $p<.05, * * p<.01, * * * p<.001$ 


\section{Hindered Career Path.}

Results of the regression analysis for the On-the-Job PD factors are presented in Table 7.11. For the prediction of Hindered Career Path, the overall model explained $29.8 \%$ of the variance: $F(18,375)=8.86, p<.001$. Entry of proximal antecedents in Step 1 resulted in a significant incremental change in $R^{2}: \Delta F(5,388)=3.95, p<.01$, explaining $5 \%$ of the variance. Beta values showed that only age and income were the significant proximal predictors. Age was a consistent significant predictor in all steps, with older participants reporting higher PD (supporting H7.4). Income as a significant predictor supported H7.5 that predicted employees with higher income would perceive less discrimination. The addition of the interaction terms in Step 2 did not result in a statistically significant $\Delta R^{2}$. In the final step, the addition of the distal factors, specifically procedural justice (supporting H7.6.1), informational justice (supporting H7.6.3), innovation (supporting H7.7.3), and organizational size (addressing RQ7.4) produced a statistically significant change in explained variance $\left(\Delta R^{2}=.24, \Delta F(9,375)=13.97, p<.001\right)$. Greater procedural and informational justice as well as greater innovation orientation are associated with less PD. Employees in larger organizations are reported lower levels of PD. Income was no longer significant in Step 3. Thereby, the predictors for hindered career path are age, procedural justice, informational justice, innovation and organizational size. 
Table 7.11

Standardized Coefficients ( $(\beta)$ in Hierarchical Regressions for the Prediction of On-the-Job Perceptions of Discrimination

\begin{tabular}{|c|c|c|c|c|c|c|c|c|c|}
\hline \multirow{3}{*}{ Predictors } & \multicolumn{3}{|c|}{ Career Path } & \multicolumn{3}{|c|}{ Work Dynamics } & \multicolumn{3}{|c|}{ Derogation } \\
\hline & \multicolumn{3}{|c|}{ Step } & \multicolumn{3}{|c|}{ Step } & \multicolumn{3}{|c|}{ Step } \\
\hline & 1 & 2 & 3 & 1 & 2 & 3 & 1 & 2 & 3 \\
\hline \multicolumn{10}{|l|}{ Proximal Influences } \\
\hline Age & $.19^{\star \star}$ & $.19^{\star \star}$ & $.19^{\star \star \star}$ & .06 & .05 & .03 & -.01 & -.01 & .02 \\
\hline Gender & .05 & -.01 & .03 & -.05 & .02 & .02 & -.03 & -.08 & -.09 \\
\hline White European (ME) & -.11 & -.13 & -.09 & .00 & $.21^{\star}$ & .14 & .08 & .15 & .05 \\
\hline Birthplace & .01 & .08 & -.01 & -.02 & .10 & -.02 & $.12^{\star}$ & .18 & .11 \\
\hline Income & $-.12^{\star}$ & $-.13^{\star}$ & -.09 & -.11 & -.10 & -.05 & -.07 & -.07 & -.10 \\
\hline \multicolumn{10}{|l|}{ 2-way/3-way Interactions } \\
\hline WEx Birthplace & & -.18 & .01 & & $-.33^{\star}$ & -.07 & & -.23 & -.09 \\
\hline WEX Gender & & .09 & .06 & & $-.27^{*}$ & $-.25^{\star}$ & & .02 & .05 \\
\hline Birthplace $\times$ Gender & & -.06 & -.01 & & -.12 & -.05 & & .03 & .09 \\
\hline WE x Birthplace $\times$ Gender & & .15 & .04 & & $.39^{\star \star}$ & .20 & & .11 & -.01 \\
\hline \multicolumn{10}{|l|}{ Distal Influences } \\
\hline JP - Procedural & & & $-.17^{\star}$ & & & -.08 & & & -.03 \\
\hline JP - Interpersonal & & & -.03 & & & $-.28^{\star \star \star}$ & & & $-.23^{\star x}$ \\
\hline JP - Informational & & & $-.19^{\star \star}$ & & & $-.13^{\star}$ & & & .10 \\
\hline JP - Distributive & & & -.02 & & & -.05 & & & -.08 \\
\hline OP - Employee Orientation & & & -.05 & & & $-.26^{\star \star *}$ & & & -.05 \\
\hline OP - Formalization & & & -.07 & & & .01 & & & -.09 \\
\hline OP - Innovation & & & $-.16^{\star \star}$ & & & .04 & & & .07 \\
\hline Sector & & & .05 & & & $.10^{\star}$ & & & -.01 \\
\hline Size & & & $.12^{\star}$ & & & -.02 & & & $-.16^{* *}$ \\
\hline$R^{2}$ & .05 & .06 & .30 & .01 & .03 & .41 & .03 & .05 & .15 \\
\hline$\Delta R^{2}$ & $.05^{\star \star}$ & .02 & $.24^{\star \star \star}$ & .01 & .02 & $.38^{\star \star \star}$ & $.03^{\star}$ & .01 & $.11^{\text {*ᄎ }}$ \\
\hline
\end{tabular}

作

Birthplace ( $0=$ NZ, $1=$ Overseas); Sector $(0=$ private, $1=$ public); $* p<.05, * * p<.01, * * * p<.001$ 


\section{Disharmonious Work Dynamics.}

In the prediction of Disharmonious Work Dynamics, a significant $R^{2}$ was observed in the overall model: $F(18,375)=14.60, \mathrm{p}<.001$ explaining $41.2 \%$ of the variance. The first two steps did not result in a statistically significant $\Delta R^{2}$ although individual variables reached significance in Step 2 (White European ethnicity; the 2-way interactions of White European and Birthplace, and White European and Gender as well as the 3-way interactions of White European and Birthplace and Gender). In the third and final step, the addition of distal variables accounted for $37.9 \%$ of the variance and a significant incremental change in $\left.R^{2:} \Delta F(9,375)=26.85, p<.001\right)$. Significant beta values were observed for the 2-way interaction of White European ethnicity and gender, interpersonal justice, informational justice, employee orientation, and sector as significant predictors of Work Dynamics. To further investigate the impact of the interaction terms in the regression model, a follow-up ANOVA was conducted, with the proximal and distal variables entered as covariates.

However, in the ANOVA, the interactions were no longer significant after accounting for all the proximal and distal variables entered as covariates first. Given that the overall regression step was not significant either, I will not interpret these interactions any further. Hence, only interpersonal justice (supporting H7.6.2), informational justice (supporting H7.6.3), employee orientation (supporting H7.7.1), and sector (addressing RQ7.5) were significant predictors of Disharmonious Work Dynamics. Higher perceptions of interpersonal justice, informational justice and employee orientation reduce 
perceived discrimination. Employees in public organizations report more discrimination.

\section{Derogation .}

The overall model predicting Derogation was significant: $F(18,375)=$ $3.77, \mathrm{p}<.001)$ and explained $15.3 \%$ of the variance. The entry of proximal variables in Step 1 resulted in a statistically significant incremental change in $R^{2}\left(\Delta R^{2}=.03, \Delta F(5,388)=2.63, p<.05\right)$ but only Birthplace was a significant predictor of Derogation supporting H7.2. This means that overseas-born employees perceived higher discrimination in the form of derogatory comments or jokes more than local-born New Zealanders. However, Birthplace was no longer significant after the addition of other predictor variables in the next regression steps. The addition of interaction terms in Step 2 did not result in a statistically significant change in $R^{2}$. In the third and final step, a significant incremental change in $R^{2}$ was observed when distal antecedents were entered into the regression equation $\left(\Delta R^{2}=.11, \Delta F(9,375)=\right.$ $5.26, p<.001)$. Significant beta values showed that only interpersonal justice (supporting H7.6.3) and organizational size (addressing RQ7.4) emerged as significant predictors of Derogation. Greater interpersonal justice is associated with less PD. Employees in larger organizations reported lower levels of PD.

The overall results (see Table 7.12) show that two proximal antecedents and seven distal antecedents emerged as significant predictors of the four PD factors. Among the proximal antecedents, age and income came up as significant predictors for Non-Recognition of Education \& Experience and age for Hindered Career Path. Among the distal factors, three justice perception dimensions, two employee practices perceptions, sector 
Table 7.12

Summary of Significant Predictors of PD Factors ( $\beta$ values)

\begin{tabular}{|c|c|c|c|c|}
\hline & $\begin{array}{c}\text { Non- } \\
\text { Recognition of } \\
\text { Educ \& } \\
\text { Experience } \\
\end{array}$ & $\begin{array}{l}\text { Hindered } \\
\text { Career } \\
\text { Path }\end{array}$ & $\begin{array}{c}\text { Disharmonious } \\
\text { Work } \\
\text { Dynamics }\end{array}$ & Derogation \\
\hline \multicolumn{5}{|l|}{ Proximal Influences } \\
\hline Age & $-.19 *$ & $.19 * * *$ & & \\
\hline Income & $-.35 * * *$ & & & \\
\hline \multicolumn{5}{|l|}{ 2-way interaction } \\
\hline \multicolumn{5}{|l|}{ WE x Gender } \\
\hline \multicolumn{5}{|l|}{ Distal Influences } \\
\hline JP - Procedural & & $-.17 *$ & & \\
\hline JP - Interpersonal & & & $-.28 * * *$ & $-.23 * *$ \\
\hline JP - Informational & $-.13 *$ & $-.19 * *$ & $-.13^{*}$ & \\
\hline OP - Employee Orientation & & & $-.26^{* * *}$ & \\
\hline OP - Innovation & & $-.16^{* *}$ & & \\
\hline Sector & & & $.10^{*}$ & \\
\hline Size & $-.10^{*}$ & $.12 *$ & & $-.16^{* *}$ \\
\hline
\end{tabular}

and organizational size came up as significant predictors of PD factors. Justice perceptions and organizational size were salient predictors for NonRecognition of Education \& Experience and Derogation. Justice perceptions, organizational practices perceptions, size and sector were significant predictors for Hindered Career Path and Disharmonious Work Dynamics.

In addition, to check for the extent to which dependencies due to nesting of samples in organizations might affect results, I first examined the intraclass correlations using a one-way ANOVA. All variables showed values exceeding .05 suggesting a nesting effect. To control for these dependencies, I ran multi-level regressions with fixed effects for all the variables and with an estimated random component that takes the nesting and dependencies (due to nesting) into account. The results of the mixed model analysis and the 
regression analysis were highly similar, with minor discrepancies (e.g. for non-recognition of education and experience, informational justice was not significant in the mixed model but was significant in the regression analysis). Hence, it was decided to retain the overall results generated from the regression analysis since nesting did not affect much the overall outcome of the analysis.

\section{Discussion}

The first objective of this study was to develop an appropriate measurement tool that would be structurally and metrically equivalent for New Zealand-born and overseas born employees. To attain this goal, measurement invariance was addressed by assessing the equivalence of the items between the two samples after determining the factor structures of the PD item responses in the EPDWS. Thus, EPDWS represents a particular important contribution to understand how employees in general, and how immigrants and New Zealand-born employees in particular, view their workplaces.

The EPDWS also attempted to capture not only current workplace barriers such as organizational practices and workplace relationships but also perceptions of barriers that are encountered during the pre-employment stage thereby considering the entire cycle of the employee's work experience. Thus, the EPDWS (i.e. through the Job-Entry and On-the-Job distinctions in PD perceptions) reflects aspects of access and treatment discrimination distinction discussed by Levitin et al. (1971), which could be both under formal and informal structures of Levine and Leonard's (1984). This framework has been 
found to be salient in the experiences of immigrants in the focus groups discussions (Chapter 4) and in the first survey in Chapter 6.

Initially, the factor structures of the three Employee Perceptions of Discrimination in the Workplace Scales (EPDWS) did not yield strong reliabilities compared to the original immigrants' perceived discrimination scale in Chapter 5. To recall, the items in the original scale were re-written to fit the perspectives of the non-immigrants. Some of the resulting factors collapsed or had very low reliabilities which prompted the decision to drop some of the factors (two for Finding Work and one for Work Conditions). These maybe due to the sampling issues since majority of the respondents were non-immigrants who may have a different outlook on the perceived discrimination issues that were generated from the themes of the immigrants' focus groups discussion. In effect, the items that were dropped are possibly reflections of the distinctiveness of visible majority (e.g. local born) and visible minority (e.g. those from Non-White European background) perceptions.

Nonetheless, the remaining four PD factors have acceptable reliability and show good validity across the migrant and non-migrant group (e.g., as indicated by the tests of structural equivalence). The psychometric properties of the remaining factors in the EPDWS shows promise as a psychometric instrument in New Zealand.

More importantly, the central objective of this study was to investigate particular factors that could influence PD in the workplace. Eleven hypotheses were proposed and five research questions were raised. Overall, seven hypotheses were supported (age, income, procedural justice, interpersonal 
justice, informational justice, employee orientation and innovation) and size and sector were identified as influencing specific PD factors. The attempt to integrate interaction terms related to proximal antecedents in order to investigate possible moderators was not as successful. Nonetheless, the regression models significantly predicted the four PD factors. Based on these results, there are key findings per PD factor, each adding to a better understanding of the influences of personal characteristics and organizational characteristics on employee perceptions of unequal treatment in organizations.

\section{Proximal Antecedents}

According to the Social Identity Theory by Tajfel and Turner (1979; 1986) and Social Categorization Theory (Turner et al., 1987), individuals classify themselves into social categories in ways that allow them to maintain a positive self-concept. One of the mechanisms underlying this theory is that people maintain a positive self-concept by favouring similar others. Hence, according to Relational Demography Theory (Tsui et al., 1992) demographic characteristics are used as parameters to determine the extent of their similarity or dissimilarity in comparison to others. The more they are similar to referent others, the more positive are their outcomes and the more they perceive they are dissimilar, the more negative are their perceptions. Hence, PD was proposed to be influenced by differences in demographic characteristics. However, in this study, the salience of social comparison along personal demographics was not as strong as the influence of organizational factors. Only age and income were found to be important proximal variables in predicting PD. 


\section{Job-Entry: Non-Recognition of education \& experience.}

A negative relationship was found for Age and Non-recognition of Education and Experience. Similarly, income was also negatively associated with this PD factor. Hence, the more the employees progress in age and the higher their income, the less they perceive discrimination in terms of nonrecognition of their educational and professional background. Perhaps, as employees mature, they will have opportunities to improve their education and skills through additional training or postgraduate studies that are either company sponsored or self-financed. Further, through the years they are able to obtain important skills that are valuable to the company unlike young employees who might have just been employed fresh from college. Increasing income may also entail lower perception of discrimination since salary increases especially in the form of merit increases are ways of recognizing employees' past performance (Cox, 2001). The ability to perform the task in turn is a reflection that the individual is qualified for the position as gauged by skills, work experiences and educational attainment.

\section{On-the-Job: Hindered Career path.}

The only significant proximal predictor of Hindered Career Path was age. Contrary to Non-recognition of Education and Experience's negative association with age, positive relationships were found for age and Hindered Career Path. Results indicate that increasing age predicted increased PD in terms of career advancement, partially supporting the proposed hypothesis on age. This is parallel to previous findings showing that upward mobility was negatively related to age (Cox \& Nkomo, 1992; Cleaveland \& Shore, 1992; 
Lawrence, 1984; Shore, Cleaveland, \& Goldberg, 2003). Younger employees are more likely to be promoted because of the longer time they can work for the organization than older employees (Cox \& Nkomo, 1992). Furthermore, age-related discrimination tends to be prevalent in higher levels of career progression (Lawrence, 1990). Moreover, although not specifically linked to career advancement, previous studies have shown that older employees face greater workplace discrimination (De Castro et al., 2008; Gee et al., 2007). Lastly, younger adults are preferred for job opportunities (Chan \& Stevens, 2001; Hirsch et al. 2000).

\section{Distal Antecedents}

Distal variables came out as better predictors of PD in the regression analysis than the personal demographic variables. These distal variables represent organizational demographics such as size and sector as well as the different systems proposed by Gelfand et al. (2005) in their Systems Model of Discrimination (SMD) framework as contexts of discrimination in organizations. Selected dimensions of perceptions of organizational justice and organizational practices perceptions representing aspects of formal/informal structures, human resource (HR) systems, organizational culture and strategy significantly influenced PD in the workplace.

\section{Job-Entry: Non-recognition of education \& experience.}

Informational justice perceptions or explanations on how outcomes are allocated were found to be a significant predictor of Non-Recognition of Education and Experience. Informational justice, as a dimension of organizational justice perceptions, is more relational in terms of reactions to 
leader-member perceptions (see Colquitt et al., 2001). The more effectively the members of organizations convey to the applicant the requirements of the job, the less discrimination is perceived in terms of recognition of qualifications.

Therefore, the individual's perceptions that his/her qualifications and/or previous work experience were considered in his/her current job largely depend on how effectively the information on qualifications were conveyed to the individual. Since informational justice is more agent-reference, then perceiving fairness in the information obtained is based upon the actions of the organizations' authority figures (Bies \& Moag, 1986; Colquitt et al., 2001). Hence, the perceptions of being recognized qualifications-wise are sourced from management officials who explain to the employee the necessary qualifications of the job like their immediate superiors and/or the human resource personnel. Based on my experience, the hiring process (either getting new employees or through internal transfers) is highly subjective, such that it is not always the case that what is written in the job descriptions serves as basis for considering people for the job.

Hence, whether the employee would see if his/her credentials are necessary to the position depends on how the supervisor or the HR personnel would explain the nature of the job, and if the employee's qualifications are indeed relevant to the job. Thus, it is not always the case that Non-Recognition of Education \& Experience will be only minimized in instances when there is a perfect match between the job description and the employee's qualification, but also on occasions when authority figures have effectively conveyed the 
necessities of the job that sometimes may not require the credentials of the employee.

Another distal factor, organizational size, also predicted individuals' perceived discriminatory treatment related educational and work experience. The result indicated that employees in bigger organizations reported lower PD related to Non-Recognition of Education \& Experience. Large organizations are characterized with rigid employment policies and hierarchical structures (Arvey et al., 1996). These hierarchical structures are usually defined by specialized job titles based on large job classifications. Baron and Pfeffer (1994) discussed that proliferation of job titles lessens the opportunity for equality comparisons since individuals are likely to compare themselves with those of similar category. Cross-category comparison is more difficult since individuals tend to interact more and know more about those in the same job category. Thereby, individuals would only compare within the same job category making it hard for them to document their true value in the organization (Baron \& Pfeffer, 1994). In large organizations therefore, perceptions of the equivalence between the individual's job and his/her qualifications are usually limited to one's job category or job title and the true extent of the individual's worth for the entire organization is minimized.

Another possible explanation is in line with Cox's (1993) descriptions of modern large organizations as becoming plural or multi-cultural. These organizations in the modern age are implementing diversity-inclusive policies as well clearer guidelines and monitoring systems regarding workplace discrimination issues (e.g. compliance to equal employment opportunity, antiharassment policies, etc.). Hence, employees are bound to perceive less 
discrimination since they observe the strict implementation of these policies in their organizations. Smaller organizations, on the other hand, have limited resources to implement such diversity-related schemes, so that diversity policies can only be successfully implemented in large organizations (R. Fischer, personal communication, June 3, 2009).

\section{On-the-Job: Hindered Career Path.}

Distal antecedents like organizational size, procedural justice perceptions, informational justice perceptions, and perceptions that organizations have innovative practices predicted Hindered Career Path or Advancement. Career advancement or promotions, a part of formal structures in organizations and implemented through HR systems, are viewed by Cox (2001) as foremost a means of filling up job vacancies and secondly as a means of developing people. He also believes that promotions are not mechanisms of reward but more of recognition that an individual has done an exceptional performance in his/her current job and has a strong potential to perform a higher level of responsibility.

Understandably, employees place a great importance to procedures that implement the promotion process since being identified as promotable would reflect on their true level of skill and performance. It was found in this study that employees perceive less discrimination in career advancement if procedures are consistent and information about the promotional criteria are likewise clear. Thus, employees view organizations with fair promotion systems as less discriminatory since these organizations would be in the position to handle promotions appropriately. Consistent with these findings, similar results were found in a study wherein the consistent application of 
promotion criteria, appropriate explanations for the criteria, and giving feedback regarding promotion decisions have been found to provide positive outcomes (Foley et al., 2002).

Another significant distal antecedent for career path is the innovation dimension of perceived organizational practices. Strategies used by organizations in their operations and approach to overall management are also regarded by the SMD model as a possible avenue for perpetuating discrimination (Gelfand et al., 2005). Organizations with innovative strategies are viewed to be more open to diversity structures hence giving more value to a diverse workforce (Cox, Lobel, \& Mcleod, 1991, Dipboye \& Collela, 2005). It appears that innovative organizations value the diversity that people bring and this is reflected in decreased PD by employees. In highly innovative organizations, diversity is an asset and can help people to progress in their career.

Lastly, organizational size was also a significant distal predictor of Hindered Career Path. Thus, the larger the organizations, the more employees perceive that they are discriminated in career advancement. Large organizations were earlier described as more bureaucratic, characterized with rigid hierarchical structures, formal job ladders and elaborate job classifications (Arvey et al., 1996; Baron \& Pfeffer, 1994; Baris et al., 2005). Furthermore, organizations with centralized, fixed policies and non-flexible seniority-based orientation structures are viewed to be susceptible to discrimination (Gelfand et al., 2005). Hierarchical-oriented job classifications would provide more layers and possibly more positions to fill-out (e.g. for an analyst position, complex job classifications would have job level distinctions 
like Analyst I, Analyst II, Analyst III, Senior Analyst, and so on). This type of structure gives employees the idea what positions to aim for but they are usually deterred by rigid seniority-based promotion decisions. Hence, the may feel that their career advancement is thereby limited and sometimes unattainable. Relative Deprivation Theory (Davis, 1959; in Crosby, 1973) explains that individuals feel unfairly treated if they want a particular item and believe that they entitled to it but it is either difficult to attain (Runciman, 1972; in Crosby, 1973) or unattainable (Gurr, 1972; in Crosby, 1973). Hence, employees may feel relatively deprived of career progression due to obstacles inherent in the formal structures of large organizations that leads to higher perceptions of discrimination.

\section{On-the-Job: Disharmonious Work dynamics.}

Interpersonal justice perceptions, informational justice perceptions, employee orientation and sector of the organization were the significant predictors for Disharmonious Work Dynamics. Work dynamics pertains more to discriminatory perceptions during interactions in the workplace, thus it is aptly predicted by the aspects of interactional justice perceptions interpersonal and informational justice perceptions. Colquitt's (2001) review of the justice literature cites interactional justice as a third dimension of organizational justice. It refers to interpersonal treatment that the individual experiences during the process of the allocation of outcomes. Greenberg (1993; in Colquitt, 2001) further suggested that interactional justice has two facets: the relational aspect in the process of allocating outcomes (interpersonal) and the manner information is disseminated regarding the 
allocation of outcomes (informational). In this study, the findings show that the more timely and accurately information is conveyed to employees and the more they perceive that they are being treated with respect by their superiors, and less likely the organization is perceived as discriminatory in workplace social interactions.

Moreover, the perceptions that an organization is more employeeoriented are associated with less PD in that workplace. The perceived climate in organizations is assumed to be a reflection of the organizational culture (Schneider, 2000). The organizational culture is manifested as shared perceptions of the policies and procedures as well as the behaviours that are rewarded by management of organizations (Reichers \& Schneider, 1990). Perceiving that organizations give value to their employees and their wellbeing generates a positive affect for employees thereby lessening any notion of discrimination. Using organizational practices as antecedent of PD is a novel approach hence no study has directly demonstrated this link previously. However, parallel findings were found in diversity climate studies wherein results show that organizations that highly favour diversity have lower levels of discrimination due to the organizations' increased concern and commitment towards proper management of a diverse workforce (Cox, 1994). Furthermore, diversity-oriented organizations were found to have higher organizational commitment and job/career satisfaction (Hicks-Clarke \& Iles, 2000). Similarly, organizations that are perceived to employee-oriented are likely to be regarded as non-discriminatory since the organizational culture observed reflects an organization that is concerned with its employees' welfare and is 
sensitive to the needs of employees regardless of their ethnic and cultural background.

Lastly, public sector organizations were perceived to be more discriminatory in terms of interactions among employees. This may be related to the perceptions of public organizations as highly bureaucratic due to their adherence to rigid and formalized policies and hierarchical structures (Baris et al., 2005). This characteristic has also been pointed out for large organizations (Arvey et al., 1996) making them more susceptible to discrimination (Gelfand et al., 2005). This is a major setback since public organizations like government agencies are supposedly the implementers of laws and public policies (e.g. equal employment opportunity, human rights laws and antidiscrimination legislations). Hence, it is expected that public organizations should be perceived as more welcoming towards employees of different backgrounds. In the absence of empirical studies that can further support these findings, this area should be a major focus of future studies in the context of workplace discrimination.

\section{On-the-Job: Derogation.}

Only two distal factors were significant predictors of perceiving derogatory remarks from co-employees, namely interpersonal perceptions of justice, and organizational size. The results indicated that interpersonal perceptions of justice predicted derogation. The less fairness is observed in terms of employee-superior interactions, the more it is associated with increasing discriminatory perceptions in the form of derogatory comments from co-employees. Interpersonal justice perceptions have been previously linked to co-worker relationships. The findings show that higher perceptions 
of interpersonal justice were strongly related to higher trust for co-workers and overall positive morale (Forret \& Love, 2008). This suggests that supervisors set the standards in the organization about appropriate interaction standards and have an important role as role models.

Furthermore, the results also show that in large organizations, the less the participants perceive that they are being derogated through racial/ethnic jokes or slurs. According to Baron and Pfeffer (1994), specialization of duties as a function of elaborated job classification in large organizations results in less interdependence of tasks. Thus, large organizations are usually depicted as distant, segregated and impersonal. Hence, it is less likely that employees will frequently encounter discrimination through derogatory comments or jokes since interaction is limited. In contrast, smaller or growing organizations are usually less segregated so that colleagues work closely together (Arvey et al, 1996). Due to the size of the organization, people in smaller organization are familiar with each other and interact more frequently although they are not necessarily intimate.

Alternatively, derogation in bigger organization is likely to be minimal if not non-existent since these organizations would have stringent policies on discrimination. As discussed earlier, large and modern organizations are described to be making an effort to be multi-cultural or embracing cultural diversity (Cox, 1993). Hence, implementation of diversity-related policies and anti-discrimination laws are well monitored. Consequently, employees would perceive less derogation due to increased acceptance of minority groups as well as being compliant to the policies. Smaller organizations are viewed to have fewer resources to implement such stringent guidelines thereby making 
large organizations as more in position to successfully implement these schemes (R. Fischer, personal communication, June 3, 2009).

\section{Limitations}

The strengths of this study are accompanied by some methodological weaknesses. First, the psychometric properties of EPDWS have been evaluated with a large proportion of NZ-born employees (68.9\%) compared to the Immigrant groups $(31.1 \%)$. Thus, it is acknowledged that there is some room for improvement in terms of representativeness of the sample and generalizing the results should be done with caution. Sampling ethnic populations has always been challenging for researchers (Cassidy, et al., 2005; Hughes, Fenton, Hine, Pilgrim, \& Tibbs, 1995). Further, the immigrant sample size approximates the census' national population but it was not statistically possible to segregate the ethnic distribution in the sample due to the small number of participants. Hence, I could not make finer distinction in terms of ethnicity (e.g. Non-Whites could be broken down into Asian, African, Middle Eastern, etc).

Most of the participants were also working in organizations hence perceptions of self-employed individuals or those that can be considered as underemployed (e.g. taxi drivers or cleaners) were not captured in this study. Moreover, there are only a few organizations in the sample thus employees from other organizations are less represented. Hence, in future studies there is a need to look into a more proportional representative sample having a more diverse ethnic, job, and age backgrounds to reassess the factor structure of the scales. It remains to be seen if the factor structure will hold across employee groups in less educated, lower position levels and more diverse age groups. 
The EPDWS was also designed based on the perceptions of discrimination of employees within the New Zealand workplace context. Although some of the items in the scale were taken from established general workplace discrimination scales from abroad, other items originate from the themes generated from the focus groups discussions that were conducted in NZ. Hence, the scale may not be applicable to participants working and living in other contexts unless some modifications are made on the scales.

All of the variables were assessed by self-report measures, raising the possibility of common methods bias. The PD process is complex since in discrimination there is a third party involved - the perpetrator (Phinney et al., 1998). Hence, the incidence of discrimination is highly subjective making selfreports to be appropriate measure. Nonetheless, this was addressed by using demographic data as control variables (age, gender and income) that are not prone to exaggerations unlike attitudes and psychological processes (Crampton \& Wagner, 1994). Further, causal relationships were inferred although this study was cross-sectional and did not capture employee perceptions within specific organizations. Thus, it is important to keep in mind that causal inferences made from cross-sectional designs are never more than inferences (Moorman, 1991). Finally, there may be omitted variables that might reflect a more complex process than what was depicted in the regression models.

\section{Suggestions for Future Research}

There are several avenues for future research that deserve to be looked into in relation to this study. First, there is a need to match management's and employees' perspectives to validate the basis for the perceptions of 
discrimination. This would help us to understand the underlying assumptions of the perceived discrimination process through examination of organizational policies, managements' perceptions and corporate cultures. For example, the distinctive aspects of organizations' corporate culture that perpetuate discriminatory policies need to be identified (e.g. marketing strategies that discriminates against the hiring of coloured people for front desks positions or policies that discriminates immigrants because of their accent). Second, other variables like personality-related variables need to be included in the examination of the influence of antecedents on PD. This will serve as a double check if perceptions are indeed rooted in the institutionalized forms of discrimination or facilitated (moderated/mediated) by person-related constructs. Third, casual mechanisms are better understood through the use of longitudinal studies. Thus, these longitudinal studies could examine the shortand long-term consequences of organizational antecedents on the workplace perceptions of employees in general and ethnic groups in particular.

Lastly, studies that identify with greater specificity how other possible antecedents (e.g. levels of education, types of occupations, length of stay in organizations) and organizational factors (e.g. leadership styles, presence of diversity and equal opportunity policies in organizations) leads to healthdebilitating employment-related discrimination are likewise needed. For instance the presence of equal opportunity policies in organizations is an increasing concern related to PD from the majority perspective. Members of the majority group may feel discriminated if there are resources in organization that are not accessible to them. It has been reported that nonbeneficiaries of Affirmative Action programs (AAP) believe that AA is 
discriminatory to them (Steeh \& Krysan, 1996). Oppositions to AAPs argue that these programs increase racial tension between preferred and nonpreferred groups (e.g. Black vs. Whites) and among preferred groups (Upper class Blacks vs. poor Blacks) (Sowell, 2004). Further, AAPs are viewed to be advantageous only to the privileged members of the minority groups at the expense of low-status majority group members (Garry, 2006). Hence, even majority members may have their own issues for claiming that they are being discriminated in what has been labelled as "reverse discrimination" or "positive discrimination" in legislations or judicial courts. Thus, organizational dynamics are a potential antecedent of discrimination.

\section{Summary and Conclusions}

Management practitioners, human resource managers, and policy making bodies need to pay attention to demographics and barriers that are subtly embedded in organizational policies that can affect employee perceptions. Special attention should be given to demographic characteristics such as age and income. It was found that the older the employees, the less they perceive discrimination in terms of their qualifications. In contrast, the older the employees, the higher their PD in terms of career advancement. In relation to income, the higher the income of employees, the less PD was reported in both qualifications and career advancement.

Moreover, organizationally-embedded factors are the strongest predictors of job-entry and especially of on-the-job perceptions. Organizational-based demographics (size and sector) and perceptions like justice perceptions (procedural, informational and interactional procedures) and organizational practices perceptions (employee-oriented or innovation- 
oriented) were also found to influence PD. Specifically, procedural justice and innovation-oriented influence PD related to structural forms like promotions or career development. The perceptions related to work relationships, on the other hand, were best predicted by relational-related distal factors like interpersonal justice, informational justice and employee-orientation. Furthermore, in larger organizations, less PD was found on recognition of qualifications and derogation while higher PD was found for career advancement. Public organizations were also viewed as more discriminatory in terms of workplace interactions.

The findings of this study provide insight on possible ways that employers and management practitioners could improve their HR systems in order to reduce if not eliminate PD. Important areas to examine are the hiring policies and compensation policies that are tapped by the findings. Hiring policies that do not recognize qualifications and experience are sources of PD. These perceptions could be minimized through entry-level hiring rates that are commensurate to qualifications and experiences as another way of recognizing employee credentials. Moreover, in the absence of actual movements in position level, salary increases are another form of progression that employees can recognize as part of their career advancement. In-house or external trainings are another plausible approach to counteract perceptions of being stagnant in one's workplace.

Furthermore, the fairness in the procedures, the manner of information dissemination and respect accorded to employees are important reflections of whether HR formal and informal structures are non-discriminatory. Finally, public organizations need to look into interactions in the workplace that 
propagate discrimination. Large organizations are better viewed as less discriminatory in terms of workplace interactions but need to be attentive to issues of career advancement that reflect higher perceived discrimination.

The next chapter, Chapter 8 , is the synthesis of all the findings of this research starting from the meta-analysis, the qualitative and the quantitative studies and the discussion of these findings including the limitations found for each study. Future areas of research are also presented in the final chapter including the practical applications. 


\section{Chapter 8 DISCUSSION AND CONCLUSIONS}

Earlier studies on race/ethnicity-related perceived discrimination have proliferated in across social science disciplines using various theoretical frameworks. In fact, no single theoretical model can unify all the relationships found between perceptions of discrimination (PD) and its correlates. Perhaps this is also a reflection of the intricate nature of the discrimination process especially from the targets' perspectives. Thus, this thesis aimed to advance the knowledge on PD at the individual-level, addressing gaps in previous research, and offering a framework that could provide an in-depth understanding about PD's nature, and its relationship with its antecedents and outcomes. The research is composed of four empirical studies - one qualitative and three quantitative studies. All studies were done in New Zealand (NZ).

This thesis makes major contributions in the advancement of knowledge in discrimination research in two different areas. The first are contributions to the general discrimination literature and the second relates more to the advancement of the PD-related research in organizational psychology.

The first major contribution is the collective findings of the metaanalysis study conducted to integrate existing studies on race/ethnicity related PD. This has not been done yet by any researcher as of the time that the metaanalysis was completed in 2007 . Hence, to the best of my knowledge this is the most comprehensive quantitative study done on race/ethnicity-related PD and no comparable meta-analytic study can be found in the literature to date. 
Secondly, a research framework was proposed for the study of PD and was used as a guide in the development of three studies. The framework has three components, namely: 1) nature of PD, 2) antecedents of PD, and 3) outcomes of PD. In the first component, statistically tested for reliability and validity measures of PD were constructed examining the nature of PD in New Zealand workplaces. These perceptions were then related to antecedents and outcomes of PD as specified in the second and third components of the framework. This research framework served as a system of organizing existing and new information about the nature of $\mathrm{PD}$ and it integrated constructs that were conceptually linked with PD.

\section{Contribution to the General Discrimination Literature}

Initially, the vast literature on discrimination was examined through a meta-analytic study (Study 1) to provide an integrative summary of the correlates of PD, identify gaps in research and to recommend possible areas of future research. Various disciplines like Sociology, Social Psychology, Acculturation Research, Management and Organizational Psychology have used different theoretical approaches in studying the nature of PD along with other constructs. The meta-analysis is important because it provided a summary of how PD relates with other concepts in different domains. Further, the results of the meta-analytic study were generally in line with most mainstream theoretical frameworks examining PD's relationship with various correlates.

Race/Ethnic Identity. First, initial findings showed that higher PD is related to stronger race/ethnic identity. This is consistent with the views of social comparison theories (Festinger, 1954, cited in Verkuyten, 1993) like 
Social Identity Theory (Tajfel \& Turner, 1979; 1986) and Social Categorization Theory (Turner et al., 1987) that explain how individuals segregate themselves into certain groups and when confronted with negative perceptions about the in-group like discrimination would identify more with similar others.

However, in the moderator analysis, the positive relationship between PD and race/ethnic identity was reversed in studies that looked into multi-level PD measurements such that higher PD was associated with weaker ethnic identity. The importance of looking at the personal and group PD perspectives and hierarchical levels of PD (e.g. peers, supervisor and systemic) are highlighted in these findings.

The meta-analytic findings, therefore, support contentions of the personal/group perceptions discrepancy. Previous researches show that personal perceptions tend to be downgraded in comparison to group perceptions (Barry \& Grilo; 2003, Dion \& Kawakami, 1996; Taylor et al., 1990; Taylor, Wright \& Ruggiero, 2001). The Discounting Hypothesis (Crocker \& Major, 1989) is a major reference framework of the personal vs. group discrepancy and could be a useful model to examine in future studies related to our study findings.

Moreover, positive associations between PD and race/ethnic identity were also reversed in studies that are field-based and have used adults and non-student samples. This implies that adult samples tended to identify less with their race/ethnic groups when perceiving discrimination in the workplace. In the case of employees, minority groups in workplaces may opt to downplay their dissimilarity since tokens (Kanter, 1977) in organizations are easily 
identifiable thus are easily segregated from the majority. Conversely, the minority employee may choose to adapt a super-ordinate identity (e.g., a marketing employee rather than an 'Asian' employee who performs marketing responsibilities). Hence, future studies interested in linking group identification and PD should also consider the multi-level aspects of PD, the study context and the characteristics of the sample.

Self-Esteem. Second initial meta-analystic result found that greater PD decreased self-esteem. This is consistent with the Rejection-Identification Model (Branscombe et al., 1999) which suggests that PD leads to exclusion and then to lower self-esteem.

Negative Well-being. In relation to negative well-being, higher PD was associated with increased negative well-being. This supports the Stress and Coping Paradigm (Ward et al., 2001) and the Acculturative Stress Model (Berry, 1990) which suggest that greater PD, regarded as a stressor, leads to negative outcomes such as problems in mental and physical health.

In addition, the impact of $\mathrm{PD}$ on negative well-being was greater for studies done in field-settings and those that used adults and non-student participants. Hence, adults experience more negative well-being symptoms when perceiving discrimination. Adolescents have multiple social networks (Clark et al., 1999) that could shield them from negative psychological impacts of discrimination (e.g. parents, relatives, peers, school programs and government policies). Furthermore, young people are viewed to be more resilient to negative experiences depending on the emotional, moral and economic support that they get from their social networks (Hawkins et al., 1992; Patterson et al., 1989; Ruggiero \& Taylor, 1997). Adults, on the other 
hand, are usually left to their own devices to cope with discriminatory practices in the workplace or in any other social context. Although there are systems in place to report the incidence of discrimination like grievance courts and courts of law, these avenues are mostly reactive rather than proactive. Hence, adults are likely to experience more the negative impacts of PD in the absence of proactive social networks combating discrimination.

Organizational Variables. Regarding organizational-related variables, greater PD was associated with less job satisfaction and more favourable attitudes towards affirmative action (AA) programs. Previous studies using Relational Demography Theory (Tsui \& O’Reilly, 1989; Tsui et al., 1992) found direct links between demographic characteristics and job outcomes (see review of Riordan et al., 2005). Hence, minority groups, in relation to the overall composition of the organization, have been found to have negative outcomes. Moreover, PD has been regarded as a proximate effect of demographic composition while organizational outcomes are distal effects, thereby emphasizing the demographic-PD-organizational outcomes continuum (Riordan et al., 2005).

The findings of this study are similar to that found by Relational Demography research since immigrants, as minorities in organizations with multiple demographic characteristics, have lower job satisfaction when perceiving higher levels of PD. Job satisfaction was found to have the highest main effect among the variables in study albeit only a few studies were retreived for the meta-analysis.

Higher PD was also found to be associated with increased favourable attitudes towards Affirmative Action programs. This can be interpreted in 
terms of the utility of these programs to shield minority groups from discriminatory practices. The association of PD with favourable attitudes towards affirmative action is stronger in studies that used multi-level measurements, done in school context and used adolescents or non-student samples.

However, the homogeneity analyses showed significant variability that indicates presence of other moderators. Future studies interested in Affirmative Action programs should place particular focus on the nonworkplace context with youth sample to investigate additional possible moderators that affect the relationship of PD and Affirmative Action attitudes. Perceptions about the implementation of Affirmative Action policies are more salient in school settings with young participants. This could be triggered by opportunities that are available for minority groups which could have both positive and negative impacts for target minority groups and usually a negative impact for the majority group. A possible negative impact for a youth from a minority group, for instance, is dealing with self and peerperceptions while obtaining scholarships in schools. Negative perceptions linger whether the minority recepient is really deserving of the award because of academic merit or just highlighting the fact that the recepient is from a lower-class, historically-oppressed groups.

Socio-economic Status. Lastly, higher PD was initially found to be negatively related to Socio-economic Status (SES). This is consistent with previous findings that showed a negative association between SES and PD (Herring et al., 1998; Taylor \& Turner, 2002). It has been noted that income disparity can be considered an indicator of economic discrimination (Schittker 
\& McLeod, 2005). Most studies looking at economic indicators found poorer economic outcomes for minority groups such as wage disparities that are reflected up to second-generation immigrant groups (e.g., Blackaby et al., 1998, 1999, 2005). The moderator analysis, however, showed a reverse relationship such that PD was positively associated with SES in adult samples and almost zero in field-contexts and in non-student samples.

Hence, it was found that the higher the SES, the greater the PD for studies that were conducted in the workplace context. It is possible that employees (who are earning - thus allowing them to be categorized into a higher level of SES compared to students who are in the school settings) are facing more discrimination since they interact daily with different groups of people in the workplace and immersed in organizations that may have systemic problems in terms of discriminatory policies. Hence, there may be a need to look at SES beyond being as a control variable and instead focus on it as a possible source or outcome of discriminatory perceptions.

Overall, the meta-analysis results showed that the strongest effect of PD was found on job satisfaction. Further, the meta-analytic review showed that there is a limited body of research that has linked PD with organizational variables. With the increasing concern placed on workplace diversity issues due to increasing globalization, the focus on organizational issues relating to PD was addressed in the succeeding studies and is considered a very relevant area of research.

Thus, the meta-analytic study contributed to the general literature by examining the strength of the relationships between PD and its correlates, examined the findings in relation to existing theoretical models and identified 
important avenues for future research. Furthermore, the findings of this metaanalytic study were mostly consistent with existing theoretical frameworks that discussed the nature of PD in several research domains. However, moderators used in the meta-analytic study changed some of the expected relationship of PD with its correlates under investigation. I offered some possible explanations and suggested new avenues for research. However, additional research is needed to explore the complex relationship in more detail across different contexts and settings.

\section{Contributions to Organizational Psychology}

For organizational psychology, this thesis made significant contributions to the advancement of knowledge related to PD in three areas. The areas correspond to the three major components of the research framework proposed in this study, namely: 1) the nature and conceptualization of PD, 2) the antecedents of PD, and 3) the outcomes of PD (see figure 8.1).

Figure 8.1

Antecedent-Consequence Research Framework for the Study of Perceived Discrimination in the Workplace

Proximal Antecedents (Perceiver's

Characteristics)

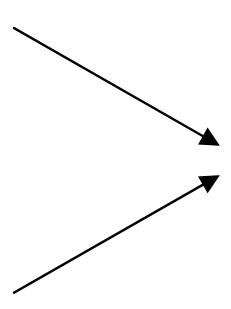

Distal Antecedents (Organizational Characteristics)
Perceptions of Discrimination in the Workplace (Nature of PD)
Organizational

Outcomes:

- Job Satisfaction

- Organizational

Commitment

- Turnover Intentions

Psychological Wellbeing Outcomes:

- Life Satisfaction

- Psychological Distress 


\section{The Nature and Measurement of Perceived Discrimination}

The first significant contribution to the organizational literature is twofold, both basically related to the core aspect of the research framework. Specifically these were a) a new conceptual framework from the qualitative study (focus groups discussions) reflecting the prevalence of PD in the different aspects of the employment cycle (pre-employment and within employment) and in the different aspects of the organizational structure (formal and informal); and b) The newly constructed scales from the surveys, the Immigrants' PD in the Workplace Scales (IPDWS) and Employees' PD in the Workplace Scales (EPDWS). The IPDWS and its modified version the EPDWS measured pre-employment and employment stages of the target groups' workplace perceptions. EPDWS was used in the investigation of antecedents highlighting the importance of the simultaneous inclusion of personal demographics and organizational factors in predicting PD. On the other hand, IPDWS was used in the prediction of outcome variables that provided valuable insights about the consequences of PD in the workplace.

\section{The Nature of PD.}

This research was able to provide a clear conceptualization of discrimination in the workplace as perceived by employees through the integration of the literature findings and results of Study 2 (Chapter 4) and Study 3a (Chapter 5). Combining qualitative and quantitative methods was a deliberate strategy used in this thesis to make best use of the advantages and to minimize the disadvantages of both research methods.

In Study 2, the focus groups discussions generated themes that converged into a conceptual model that supported and advanced the existing 
conceptualization of discrimination offered by organizational psychologists. The synthesis of the themes that converged into a 4-quadrant model (see Table 8.1) is important in understanding immigrants' experiences of discrimination in the workplace. This approach of systematically organizing themes that reflect the prevalence of PD in different aspects of the employment process has not been directly addressed before. This model, however, approximates early theoretical models by organizational psychologists in the field of gender and lesbian/gay discrimination research.

The Job-Entry and On-the Job aspects of the model are similar to Access and Treatment Discrimination described by Levitin et al. (1971) as two components of gender discrimination in organizations. Moreover, the Formal and Informal facets of the model are comparable to the Formal and Informal Discrimination reported in the Gay/Lesbian study by Levine and Leonard (1984). Chung (2001) suggested in his conceptual paper that the Access and Treatment Discrimination by Levitin et al. (1971) can be categorized under Formal Discrimination proposed by Levine and Leonard (1984). My model therefore is an expansion of Chung's (2001) analysis. Based on the themes from immigrants' experiences, access (Job-Entry) and treatment On-the-Job discrimination were not only evident in the formal discrimination category but extended to the informal discrimination domain.

This model therefore is a significant contribution to understanding the concept of discrimination in the workplace settings from employed immigrants' perspectives. Moreover, the conceptual model generated from the focus groups discussion' themes reflected the robustness of the components of discrimination suggested y Levitin et al. (1971) and Levine and Leonard 
Table 8.1

Perceptions of Workplace Discrimination from the FGDs*

\begin{tabular}{|c|c|c|}
\hline & \multicolumn{2}{|c|}{ Phases of Employment } \\
\hline & Job-Entry & On-the-Job \\
\hline & (Q1) & (Q3) \\
\hline \multirow[t]{4}{*}{ Formal Structures } & Language/Accent & Language Accent \\
\hline & Overseas qualifications not recognized & Mismatch (Job vs. Qualifications) \\
\hline & "Kiwi" experience & Inequity in Pay \\
\hline & (Q2) & (Q4) \\
\hline \multirow[t]{2}{*}{ Informal Structures } & Reluctance towards colored people & Distrust for colored people \\
\hline & Network/Knowing people & \\
\hline
\end{tabular}

(1984). Additionally, there are supplementary findings in the focus groups' model that were not found in gender and gay/lesbian studies. Hence, this means that immigrants face additional hardships in the workplace in the form of discriminatory perceptions linked to work relationships or interactions that are largely an offshoot of being different from the majority. Themes that relate to "reluctance towards coloured people" or "distrust for coloured people" mean that immigrants perceive discrimination related to their race/ethnic background on top of their status as immigrants and other demographic characteristics such as gender and age. Immigrants, therefore, are important sources in investigations for the initial conceptualization of PD since they embody multiple characteristics that are susceptible to discrimination. Their discriminatory perceptions, influenced by their multiple demographic characteristics, capture a wide range of perspectives such that the resulting 
framework can be applied to any target group (e.g., ethnic, gender, age, immigrants, etc).

\section{Measurement of PD.}

The second level in the investigation on the nature of PD was conducted through a survey (Study 3-A). Prior to the construction of the PD scales, the race/ethnic discrimination literature were reviewed for instruments that could be directly adapted for the quantitative studies. However, the search to find a single scale for inclusion in this thesis proved to be futile as explained in detail at Chapter 5. No existing PD instrument could represent the 4-quadrant model (see Table 8.1) that was found in the focus groups themes. Hence, the author embarked on the construction of new PD scales using the themes from the focus groups and selecting items from several existing PD instruments in gender studies and organizational research.

Immigrants' Perceptions of Discrimination in the Workplace Scales. Thus, three scales were initially constructed, namely Finding Work, Work Conditions and Work Relationships. These three scales were collectively called Immigrants Perceptions of Discrimination in the Workplace Scales (IPDWS). Finding Work contained items that represent PD in formal and informal structures during job-hunting stage. Work Conditions contained items the reflect perceptions related to formal structures in organizations like promotions and benefits policies. Lastly Work Relationships represents perceptions of the quality of interactions in the workplace by measuring supervisor-subordinate relationships and co-worker interactions. Table 8.2 is an illustration how IPDWS items fit in the model generated from the immigrants' focus groups discussions. 


\section{Table 8.2}

\section{IPDWS ${ }^{*}$ Fit in the FGD Model}

\begin{tabular}{|c|c|c|}
\hline & \multicolumn{2}{|c|}{ Phases of Employment } \\
\hline & Job-Entry & On-the-Job \\
\hline Formal Structures & $\begin{array}{l}\text { Finding Work - items that affect hiring } \\
\text { decisions such as educational } \\
\text { qualifications, work experience, } \\
\text { accent, looks, language, e.g. } \\
\text { Recognition of Education and Work } \\
\text { Experience, Local Requirements }\end{array}$ & $\begin{array}{l}\text { Work Conditions - items } \\
\text { related to promotion, trainings, } \\
\text { benefits and compensation }\end{array}$ \\
\hline Informal Structures & $\begin{array}{l}\text { Finding Work - items that necessitate the } \\
\text { need to know people in } \\
\text { organizations or networking }\end{array}$ & $\begin{array}{l}\text { Work Relationships - items } \\
\text { related to work relationships, } \\
\text { derogatory jokes, interactions }\end{array}$ \\
\hline
\end{tabular}

Each scale of the IPDWS-pilot or original version was subjected to principal component analysis (examination of primary factors or the horizontal investigation of the factors) and generated four factors for Finding Work, three factors for Work Conditions and three factors for Work Relationships. See Table 8.3 for the fit of the ten PD factors in the FGD model. The psychometric properties of the resulting PD factors showed the instruments to be valid and reliable.

Table 8.3

IPDWS's* (10 factors) Fit in the FGD Model

\begin{tabular}{|c|c|c|}
\hline & \multicolumn{2}{|c|}{ Phases of Employment } \\
\hline & Job-Entry & On-the-Job \\
\hline Formal Structures & $\begin{array}{ll}\text { Finding Work } \\
-\quad \text { Non-local } \\
-\quad \text { Non-Recognition of education } \\
\quad \text { \& experience } \\
-\quad \text { Local Requirements }\end{array}$ & $\begin{array}{l}\text { Work Conditions } \\
\text { - Hindered Career Path \& Unequal } \\
\quad \text { Opportunity } \\
\text { - Inadequate Compensation } \\
\text { - Differing Treatment }\end{array}$ \\
\hline Informal Structures & $\begin{array}{l}\text { Finding Work } \\
\text { - Networking }\end{array}$ & $\begin{array}{l}\text { Work Relationships } \\
\text { - Exclusion } \\
\text { - Derogation } \\
\text { - Disharmonious Work Dynamics }\end{array}$ \\
\hline
\end{tabular}


The ten PD factors were further subjected to another principal component analysis to determine a higher order factor structure (examination of a vertical structure). Two secondary factors emerged wherein all factors from Finding Work Scale converged under Job-Entry and all factors from the Work Condition and Work Relationships Scales combined under the On-theJob. Since there were just two interpretable factors, this suggests that the distinction between job entry versus on-the-job is more salient to individuals working in organizations. Additional inspection of the factors revealed that some factors still fell either under formal or informal structures.

These scales represent another important contribution to the methodological aspect of discrimination research since this is a valid measure of immigrants' PD in the workplace. Moreover, the scales capture different components of discrimination that provide a more comprehensive investigation of possible discriminatory facets in organizations. The conceptualization of the scales was based on the basic experiences of immigrants as well as theoretical approach. Hence the items in these scales reflect recent perceptions of immigrants in the age of globalization but are also representations of discriminatory perceptions from previous scales that remain relevant despite legal and societal pressures to curb discrimination.

Employees' Perceptions of Discrimination in the Workplace Scales. The IPDWS items were modified to fit non-immigrant employees' perceptions. The modified three scales were subsequently named Employees' Perceptions of Discrimination in the Workplace Scales (EPDWS). Each scale of the EPDWS was also subjected to principal component analysis and generated a total of four interpretable factors that focused on individual 
perceptions - Finding Work (1), Work Conditions (1) and Work Relationships (2). The resulting factors show that employees PD are less complex than immigrants' PD as reflected by the fewer factors. Some of the items in EPDWS did not converge in a meaningful fashion similar to that of the IPDWS. See Table 8.4 for the fit of the four PD factors in the FGD model. The psychometric properties of the resulting PD factors also showed that the EPDWS are valid and reliable.

Table 8.4

EPDWS's* (4 factors) Fit in the FGD Model

\begin{tabular}{lll}
\hline & \multicolumn{2}{c}{ Phases of Employment } \\
\cline { 2 - 3 } Formal Structures & \multicolumn{1}{c}{ Job-Entry } & \multicolumn{1}{c}{ On-the-Job } \\
\hline & $\begin{array}{l}\text { Finding Work } \\
\text { Non-Recognition of education } \\
\text { \& experience }\end{array}$ & $\begin{array}{l}\text { Work Conditions } \\
\text { - Hindered Career Path }\end{array}$ \\
Informal Structures & \multicolumn{1}{l}{$\begin{array}{l}\text { Work Relationships } \\
\text { - Derogation } \\
\text { - Disharmonious Work Dynamics }\end{array}$} \\
\hline $\begin{array}{l}\text { * EPDWS - Employee Perceptions of Discrimination in the Workplace Scales; FGD - focus } \\
\text { groups discussions }\end{array}$
\end{tabular}

The EPDWS factor structures were further tested for structural equivalence. This is another significant contribution to the organizational psychology literature since the scales have passed the structural equivalence standards and thereby can be used for the general population. Hence, the items in the scales have the same meaning for both immigrants and non-immigrants (e.g. native-born) employees. This is a useful tool in the investigation of PD using mixed samples of employees coming from different ethnic backgrounds and different nationality status. 


\section{Summary}

The investigation on the nature of PD was done through a combination of quantitative and qualitative methods. The themes generated from the focus groups discussions became important bases for constructing scales that reflect immigrants' and employees' perceptions of discrimination in the workplace. The themes according to their classifications were represented in the items of the scales. The focus groups' quadrant model was easily replicated in the factor structures of the immigrants PD scales (IPDWS). However, some items were rephrased in the second scale to fit the non-immigrant participants in the employees PD scales (EPDWS) and subsequently some factors disappeared (see Figure 8.2). This means that there are perceptions in the workplace that are salient only to immigrants.

Factors that were salient for immigrants were being "different" (physically, having an accent), requirements like having "kiwi" experience, and networking or the need to know people in organizations. Most of the factors that remained for the EPDWS were related to perceptions about the policies and procedures of the organizations (e.g. hiring policies, career development and pay) and workplace relationships (pleasant work environment). These are concerns that could be expected to be perceptible by all employees without looking at race/ethnic differences or the possible distinctions of "visible vs non-visible" population sample. Nonetheless, these findings are preliminary and the studies are exploratory. 
Figure 8.2

Summary of Findings from the Qualitative Study and Factor Structures from the Immigrant and Mixed Samples Surveys

\begin{tabular}{|c|c|c|c|c|}
\hline & & $\begin{array}{l}\text { Perceptions of } \\
\text { Discrimination in the } \\
\text { Workplace } \\
\text { (Nature of PD) }\end{array}$ & & \\
\hline & \multicolumn{2}{|c|}{$\begin{array}{l}\text { The Nature of Perceived Discrimination } \\
\text { In New Zealand Workplaces }\end{array}$} & & \\
\hline GROUPS & $\begin{array}{l}\text { Q1 } \\
\text { (Job Entry/ } \\
\text { Formal) }\end{array}$ & $\begin{array}{l}\text { Q2 } \\
\text { (Job-Entry/ } \\
\text { Informal) }\end{array}$ & $\begin{array}{c}\text { Q3 } \\
\text { (On-the-Job/ } \\
\text { Formal) }\end{array}$ & $\begin{array}{c}\text { Q4 } \\
\text { (On-the-Job/ } \\
\text { Informal) }\end{array}$ \\
\hline $\begin{array}{l}\text { Focus Groups } \\
\text { Discussions }\end{array}$ & $\begin{array}{l}\text { Language/Accent } \\
\text { Overseas } \\
\text { Qualifications not } \\
\text { recognized } \\
\text { "Kiwi" experience }\end{array}$ & $\begin{array}{l}\text { Reluctance towards } \\
\text { coloured people } \\
\text { Networking/ } \\
\text { Knowing people }\end{array}$ & $\begin{array}{l}\text { Language/Accent } \\
\text { Mismatch (Job vs. } \\
\text { Qualifications) } \\
\text { Inequity in Pay }\end{array}$ & $\begin{array}{l}\text { Distrust for } \\
\text { coloured people }\end{array}$ \\
\hline $\begin{array}{l}\text { Immigrants } \\
\text { (IPDWS) }\end{array}$ & $\begin{array}{l}\text { Non-local } \\
\text { Non-Recognition of } \\
\quad \text { educ \& } \\
\quad \text { experience } \\
\text { Local Requirements }\end{array}$ & Networking & $\begin{array}{l}\text { Hindered Career } \\
\text { Path \& Unequal } \\
\text { Opportunity } \\
\text { Inadequate } \\
\text { Compensation } \\
\text { Differing Treatment }\end{array}$ & $\begin{array}{l}\text { Exclusion } \\
\text { Derogation } \\
\text { Disharmonious } \\
\text { Work } \\
\text { Dynamics }\end{array}$ \\
\hline $\begin{array}{l}\text { Mixed Sample } \\
\text { (EPDWS) }\end{array}$ & $\begin{array}{l}\text { Non-recognition of } \\
\text { educ \& } \\
\text { experience }\end{array}$ & & $\begin{array}{l}\text { Hindered Career } \\
\text { Path }\end{array}$ & $\begin{array}{l}\text { Derogation } \\
\text { Disharmonious } \\
\quad \text { Work } \\
\text { Dynamics }\end{array}$ \\
\hline
\end{tabular}

\section{Antecedents of Perceived Discrimination}

The second contribution to the organizational literature pertains to the analysis of what antecedent variable(s) best predict(s) PD in the research framework. The "upstream" analysis (Schnittker \& McLeod, 2005) of PD conducted through the careful examination of antecedents contributed to the advancement of knowledge about which particular factors are most influential 
in predicting PD. This investigation is important because if you want to solve a problem, you need to know the source of the problem so you can curtail it.

Not all possible proximal (personal) and distal (external/environmental) factors can be included in any single study, therefore a selection of two sets of key variables were included in this study and were compared for their predictive ability in relation to PD. This is a novel way of examining antecedents of PD in the workplace. No existing study in either organizational or discrimination literature has simultaneously included both proximal and distal variables that were used in the current study. Hence, the identification of the most salient antecedents of PD is another important contribution to the organizational psychology literature.

Proximal Antecedents. Proximal antecedents refer to perceivers' demographics such as age, gender, birthplace (Immigrant or NZ-born), ethnicity (European White or Non-White) and income. Findings for proximal antecedents show that only age and income were significant predictors of selected PD factors both in Job-Entry and On-the-Job. Older employees and those with higher incomes had lower PD in relation to recognition of education and experience. Moreover, older employees tended to perceive more PD related to career development. Hence, older employees seemed to have less access to higher positions in the organizations. This is consistent with previous findings linking increasing age with fewer opportunities for career advancement (Cox \& Nkomo, 1992; Cleaveland \& Shore, 1992; Lawrence, 1984; Shore, Cleaveland, \& Goldberg, 2003). Overall, the limited role of demographics in influencing PD is not consistent with predictions derived from the literature on Relational Demography (Tsui et al., 1992), Social 
Identity Theory (Tajfel \& Turner, 1979; 1986) and Social Categorization Theory (Turner et al., 1987) that highlight the importance of demographic characteristics as bases for social comparison that eventually leads to negative personal outcomes.

Distal Antecedents. The distal antecedents in this study were organizational demographics (size and sector) and employee perceptions of the processes and structures within the organization. This included the four dimensions of organizational justice (procedural, informational, interpersonal and distributive justice) and the three dimensions of organizational practices (employee orientation, formalization and innovation). The distal variables represented aspects of the different organizational systems that serve as contexts of workplace discrimination as reflected in the Systems Model of Discrimination proposed by Gelfand et al. (2005).

In terms of organizational size, larger organizations were found to have lower PD in terms of recognizing qualifications and hearing derogatory comments from co-workers. On the other hand, bigger organizations were perceived to be more discriminatory in terms of career advancement. Public organizations were also perceived to be more discriminatory in terms of work dynamics or social interactions at work. Hence, no particular organizational size and sector can be pointed out as free from being viewed as discriminatory.

Related to other distal antecedents, informational justice was a significant predictor of both Job-Entry and On-the-Job PD factors. Specifically, employees who perceive that they get less timely and less accurate information about particular outcomes in their workplace are more likely to report greater $\mathrm{PD}$ in terms of recognition of education and 
experience, career advancement and social interactions. Hence, there is a need for members of the management team to convey information about procedures and processes (e.g., the needs and requirements for the vacant positions, appeal mechanisms, general work procedures) more effectively so that less discrimination is perceived by employees. Moreover, interpersonal justice was found to be related to PD (in terms of work relationships).

The associations found between the two justice perceptions (informational and interpersonal) and work relationship factors of PD are consistent with the definition of interactional justice. Interactional justice was found to have two dimensions - interpersonal and informational. Both perceptions are related to the quality of relationships in organizations (Colquitt, 2001). Organizational structure-driven perceptions of organizational justice like procedural justice (Colquitt, 2001) best predicted PD regarding career advancement. Determination of career advancements in organizations is in turn a function of formal structures and HR policies on promotions in organizations; as such, the findings are in line with the justice literature (Colquitt, 2001; Colquitt et al., 2001).

The perceptions of organizational practices also emerged as significant contextual factors in predicting PD specifically in On-the-Job factors. The strategy of the organization can influence perceptions related to upward movement in organizations, so that higher PD was predicted by perceptions that organizations are not innovation-oriented. This is consistent with the view that organizations pursuing innovation strategies are more open to diversity policies thus recognizing the importance on a diverse workforce (Cox et al., 1991; Dipboye \& Collela, 2005). 
Moreover, perceptions that organizations are oriented more toward employees, or perceptions that management are genuinely concerned about their employees' welfare, predicted lower discriminatory perceptions in work relationships. These findings are another important contribution to the organizational culture and discrimination literature. This is the first time that organizational practices variables have been related to PD with the use of scales measuring new dimensions of organizational culture. Hence, this is a novel approach in looking at the relationship of PD with the organization's culture and strategies.

\section{Summary}

Proximal and distal variables were found to be significant predictors both for Job-Entry and On-the-Job PD factors. However, overall findings (see Figure 8.3) show that distal variables measuring aspects of the Systems Model of Discrimination (SMD) of Gelfand et al. (2005) were better predictors of PD than perceivers' demographics as proposed by Relational Demograpy Theory (Riordan, 2005; Tsui et al., 1992) and related theories. Thus, the study's findings strongly support Gelfand and colleagues' (2005) assertion that organizational systems are important sources of discriminatory practices.

Relevant aspects of SMD (Gelfand et al., 2005), namely formal and informal structures, HR systems and organizational culture, can be contexts of perceptions of justice. Thus, employees may perceive unfairness in procedures related to promotions, performance management or opportunities for training and development. They perceive discrimination in terms of how the procedures are implemented, being communicated and executed among employees and across organizations. 
Figure 8.3

Significant Antecedents/Predictors of Perceived Discrimination Mixed Sample

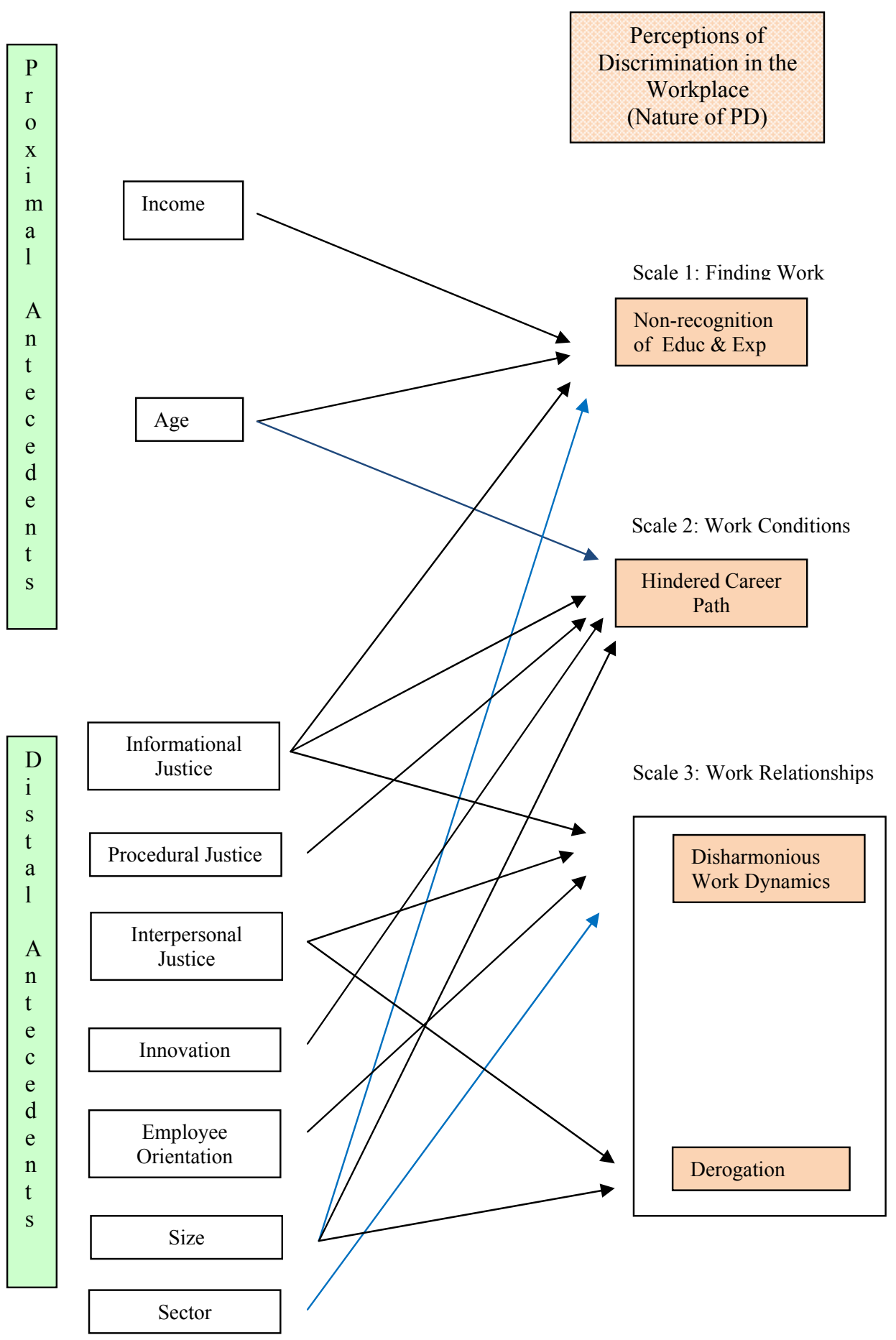

Note: Blue lines and black lines indicate positive and negative relationships, respectively. 
Furthermore, the respondents also perceive discrimination in relation to manner of executing the procedures reflecting the quality of social interactions. For instance, interpersonal justice was a significant predictor of disharmonious work dynamics and derogations. Hence, the way higher management relates to subordinates has an effect on the actual interactions within the shop floor. Thus, the different aspects of organizational structure should be carefully examined for possible areas for improvements.

\section{Perceived Discrimination and Outcomes}

The final important contribution to the organizational literature relates to the outcomes aspect of the research framework. This was the investigation of the relationship of PD with organizational and psychological well-being variables. Considered as the "downstream" analysis (Schnittker \& McLeod, 2005) of the PD process, Study 3-B (Chapter 6) looked into the consequences of PD in the workplace using a sample of working immigrants.

Job Outcomes. The framework looked at three organizational variables as outcomes of PD namely job satisfaction, organizational commitment (affective, continuance and normative) and turnover intentions. The results revealed that On-the-Job PD factors were consistent significant predictors of organizational outcomes.

Specifically, higher PD in areas relating to career advancement, receiving adequate compensation and equal treatment of policies regardless of ethnic/cultural background were the best predictors of low job satisfaction. Among the outcomes, job satisfaction was strongly associated with On-theJob PD factors. This parallels the result of the meta-analysis that also showed 
a strong PD effect on job satisfaction. In Study 4, specific PD factors were identified that best predict job satisfaction. These were discriminatory perceptions related to organizational structures that implement policies and practices on career advancement and compensation. Hence, these areas should be carefully examined for discriminatory practices that are embedded in these systems since these types of PD influence the degree of people's satisfaction in their jobs. Job satisfactio has been found to be related to positive (e.g. commitment to organization) and negative organizational outcomes (e.g. turnover) that are important to successful organizational functioning (Mathieu \& Zajac, 1990; Mowday et al., 1982). Hence, a particular focus is warranted on this issue in organizational research.

Moreover, lower emotional attachment to one's workplace was predicted by higher PD in terms of career advancement and exclusion. Higher intentions to leave were likewise predicted by greater perceptions of exclusion. Hence, attachment to organizations in terms of commitment and intent to stay are affected by feelings of being excluded in workplace interactions. Hence, feelings of belonging will also result in the decision to belong or to stay in an organization.

One surprising finding is the positive relationship between nonrecognition of education and experience and continuance commitment. This supports the 'lack of alternative' component of continuance commitment as proposed by McGee and Ford (1987). Thus, if the employee feels that his/her qualifications are not being recognized, the perceived cost of leaving the organization is also high since other employer may be treating them the same way. Continuance commitment is usually treated as a uni-dimensional 
construct in most organizational commitment studies. Hence, the findings of this study warrant the further investigation of the subcomponents of continuance commitment as suggested by Meyer et al. (2003). It is worth examining if the emergence of the sub-components of continuance commitment is also relevant in non-discrimination studies.

Well-being Measures. The study also examined two psychological well-being measures, life satisfaction and psychological distress, as positive and negative indicators of employee adaptation in the workplace, respectively. Similar to the organizational outcomes, only On-the-Job PD factors emerged as significant predictors of the two outcome variables. Specifically, perceptions of derogation were significant predictors of lowered life satisfaction and greater psychological distress.

Perceptions of being derogated are related to increased psychological distress and less life satisfaction. Ethnic minority groups are subjected to derogatory attitudes like racial or ethnic slurs or jokes that demean the cultural characteristics of the group. Similar to the findings in the meta-analysis, PD in the form of derogatory perceptions can be viewed as a stressor that eventually leads to negative outcomes. This is also consistent with the Stress and Coping Paradigm (Ward et al., 2001) and the Acculturative Stress Model (Berry, 1990) that suggests a positive association between greater PD and increased problems in psychological health of discriminated target groups.

Finally, the negative impact of demographic variables also deserves some attention. Age was significantly related to turnover intention regardless of immigrants' PD. The findings suggest that older immigrants are reluctant to leave their organization because they may have a hard time finding other 
meaningful employment. As New Zealand is a small country, hence based on economies of scale there will be fewer opportunities in here compared to bigger countries like Australia. Moreover, being an immigrant is already a reason for experiencing difficulty in gaining employment (Bedford et al., 2001; Mace et al., 2005; Podsiadlowski \& Ward, forthcoming.; Trlin et al., 1999; Ward \& Masgoret, 2007) and being underemployed (Mace et al., 2005; NZ Department of Labour, 2005; 2006; Ward \& Masgoret, 2007). Thus, older age is regarded as another hindrance in securing another job. The previous meta-analysis on organizational commitment and turnover also showed that older employees were found to be less likely to leave, even if they have low commitment to the organization due to structural bonds (e.g. compensation) (Cohen, 1993). Older immigrants may have similar needs and may face more difficulties to obtain meaningful jobs due to their immigrant status.

\section{Summary}

Perceptions of discrimination during the Job-Entry stage does not appear to have long-term consequences for immigrants since their job-entry perceptions (Finding Work) were unrelated to any psychological and organizational outcomes. This result contrasts with On-the-Job PD factors that were found to be significantly related to the outcomes (see Figure 8.4). Hence, in relation to organizational outcomes, particular attention should be given to organizational policies and practices related to career advancement of immigrants and the uniform implementation of policies regardless of ethnic/cultural background since these are associated with increased job satisfaction and emotional attachment to organizations. 
Similarly, factors related to PD arising from work relationships were found to be important for job outcomes as well as psychological well-being outcomes. Those who feel excluded and derogated were also more likely to have less satisfaction in life and perceive more psychological distress. Thus, not only work relationship factors affect job outcomes, they also have an impact on psychological well-being. It is thereby important to give focus to work relationship items as well because they have significant influence on immigrant outcomes.

Race/Ethnicity-related discrimination studies in the organizational context have mostly focused on economic effects of discrimination like wages and rates of unemployment/underemployment. Further, there are only a handful of studies that have looked into organizational outcomes of employees in general. Thus, an important contribution of this study is the systematic investigation of PD's impact on both job and psychological well-being outcomes. The investigation of these two sets of outcomes in one model involving not only employees but also another specific group of targets employed immigrants - adds novelty to this approach for investigating PD outcomes. The investigation of the three levels of organizational commitment is also a new approach to the stud of PD. Future studies should follow a similar approach through the careful examination of constructs with several dimensions so that specific dimensions of a construct will be highlighted. 
Figure 8.4

Significant Outcomes Predicted by Perceived Discrimination Immigrant Sample

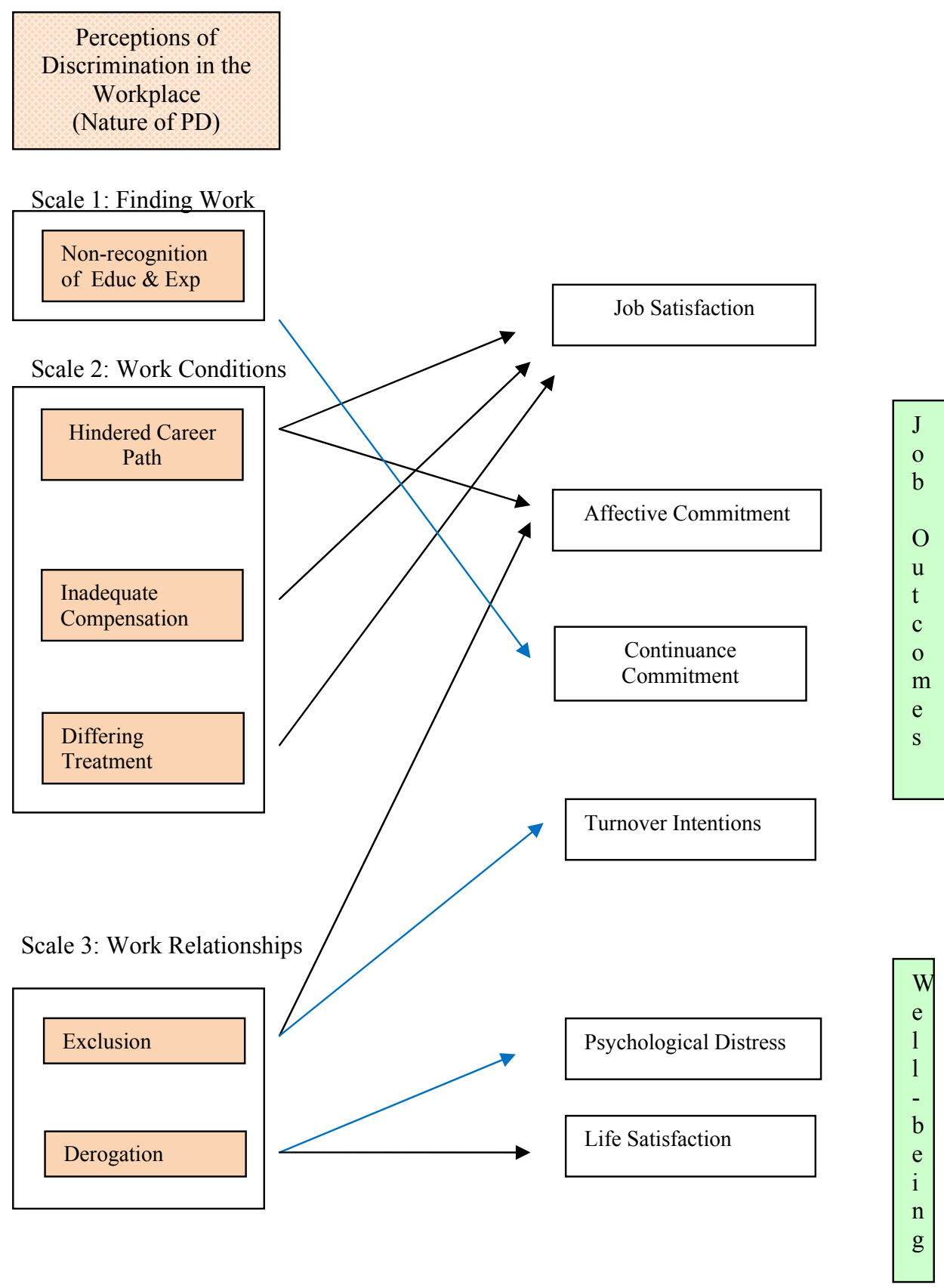

Note: Blue lines and black lines indicate positive and negative relationships, respectively. 


\section{Limitations and Future Research}

The newly constructed scales used in this research to measure PD of immigrants have not been subjected to confirmatory factor analysis. Hence it is suggested that further analyses should be conducted on the scales to confirm their factor structures and to ensure their reliability and validity across different samples.

Moreover, generalizability of the results should be treated with caution since the examination of the antecedents and the outcomes of PD were done through surveys and correlational research. Proposed causal relationships were examined through correlational design; hence, the causality of the relationships among the findings can not be tested. Nonetheless, future studies can use experimental or longitudinal research methods to address this limitation. Furthermore future studies can use different data collection methodologies such as direct observation, peer-rated observation, or collection of actual organizational reports such as performance ratings, actual turn-over data and other explicit and implicit measures of organizational outcomes as well as perceptions of discrimination.

The generalizability of the results is also limited because the samples used in the surveys were not representative in terms of ethnicity and immigrant status. Though the ethnicities of the respondents approximated the actual population distribution in New Zealand, an in-depth examination of differences in ethnicity was not undertaken other than comparing European White and Non White groups. This reflected inherent difficulties obtaining 
data in the field setting. Hence, future studies should make an explicit attempt to gather more immigrant participants.

Finally, the findings were generated using New Zealand-based samples. Therefore, the applicability of the results may be limited to New Zealand only. Future studies should look into the PD's factor structures and their relationship to different correlates across a broader range of national contexts. Organizations are experiencing increasing diversity due to increasing globalization. Hence the issues that were addressed in this research may be applicable to a wider international labour market, but this transfer needs to be tested.

\section{Implications and Practical Applications for Organizations and Policy Makers}

There are a number of implications and practical applications identified in the research that may be useful to organizations and to policy making bodies interested in eliminating workplace discrimination.

First, the findings in this research show that public organizations are viewed as more discriminatory than organizations in the private sector, specifically in terms of work relationships. Hence, there is a need to examine closely the social interactions in public organizations that serve as source of PD. Government agencies are supposedly the primary implementers of antidiscrimination laws and equal opportunity employment practices, but the actual implementation of these policies does not always trickle down to the members of the organization - its employees.

These findings have several implications for New Zealand's public organizations. First, public organizations adherence to the government's 
policy of embracing diversity may be too lax. The programs may be in place, but there is little or no compliance with these programs. It may also be that because of these policies expectations are particularly high and therefore, employees are more sensitive which leads to higher PD. Conversely, the implementation of these programs may be too rigid so that some employees may already see the presence of anti-discriminatory policies as a form of reverse discrimination.

Secondly, members of management and policy-making bodies should look closely at the differences between small and large organizations. Larger organizations are perceived as more accepting in terms of qualifications and experience, as well as less likely to condone derogatory comments. Larger organizations are in the position (financially and logistically) to implement and monitor stricter rules on anti-discrimination policies compared to smaller organizations. Hence, it is more likely that larger organizations are viewed as less discriminatory since these policies are highly visible and actively implemented.

However, larger organizations are also perceived to be discriminatory in terms of career advancement. This is alarming because most of the participating organizations in this study are from medium to large organizations. Hence, these perceptions are prevalent in current organizations. Dissatisfaction with these organizations in terms of fewer opportunities for advancement would heighten the problems of New Zealand in terms of outward flow of talents since employees may look for more lucrative employment overseas. The emigration of skilled and educated New Zealanders is a problem that needs to be addressed not only in organizations but also by 
policy-making bodies. This is an additional problem in the tight labour market during the duration of the studies.

Third, there is also a need to look into organizations and examine the systems that may be perpetuating discrimination - either formal or informal structures, HR policies or the culture within organizations. Based on the findings, organizational systems play a more significant role in predicting perceived discrimination than personal demographics. The possibility of eliminating workplace discrimination is high in this regard since organizational systems and practices (e.g., hiring policies, promotional guidelines, training opportunities, and employee-management interactive workshops) can be modified or changed, unlike personal characteristics that usually serve as the basis for discrimination. Moreover, in the workplace context, the problem of solving discrimination may not be as intricate as solving discrimination in the general social context since the systems perpetuating discrimination can be easily identified (e.g. systems-related or management-related) if influential people in management would allow the resolution of this problem.

There is a need to look specifically into the formal systems of organizations like promotional policies, career progression, compensation, performance management, and supervisor-subordinate dynamics because these are the areas that perpetuate perceptions of discrimination. Thus, organizations could conduct a policy audit and look at these areas that perpetuate higher perceptions of discrimination. This policy audit could be done nation-wide and across industries with support from the national government so that appropriate legal sanctions can be acted upon immediately. Alternatively, the 
government should also be ready to provide rewards and incentives for organizations who show their best practices in terms of eliminating discrimination practices.

Finally, governments of host countries like New Zealand that accept skilled immigrants in order to augment a tight labour market, should look into the needs of immigrant employees. Results of this research show that perceived discrimination stems from various experiences in their new host country including non-recognition of educational qualifications and work experience, inability to see a definite path in career development and work relationships that lead to dissatisfaction, less commitment and negative psychological well-being.

The negative impact of the unfavourable outcomes may not be felt immediately by organizations in terms of lower performance and increased employee health costs. However, these negative perceptions will definitely have long-term impacts both for the organizations and the economy of the country. Immigrants may opt to leave the country and look for more lucrative opportunities outside of New Zealand. The eventual departures of immigrants defeat the purpose of enticing skilled people from overseas to come to New Zealand to work and live for indefinite periods of time in order to help the growing economy. Otherwise, New Zealand would just be a staging point for skilled immigrants who left their home countries for better economic opportunities overseas. Thereby, a proactive approach should be taken by both government agencies and private organizations to address PD that originated from the challenges that immigrants face in their working life in the host country. 
For instance, governing bodies like New Zealand Qualifications Authority, the New Zealand Immigration Service and Department of Labour should have a periodical review of the outcomes of the immigrants that have been accepted in different organizations in order to monitor their progress, in particular their problems and concerns. Furthermore, there should be proactive assistance programs that could be provided for the immigrants that could prevent negative employment experiences (e.g. free training in terms of preemployment interviews, exam preparations, and resume preparations). An association of employers could conduct these free trainings so that contents of trainings are current and address specific needs of existing organizations.

\section{Conclusions}

Is discrimination really as common as the air we breathe? It appears that discrimination is still present in more subtle and unobtrusive forms than the severe way it has been manifested in the past. Similarly, discrimination research has come a long way from assisting legal mandates on antidiscrimination and human rights and measuring economic determinants of discrimination to more sophisticated studies on the psychological antecedents and consequences of PD. I am privileged to have contributed to the expansion of knowledge on the intricate nature of PD using a holistic and integrative approach that synthesizes previous research and leads to practical applications.

In modern times, integration of different countries' labour markets due to globalization has paved the way to placing importance on increasing diversity in the workplace. It has also placed increased concern on attracting and retaining highly skilled human resources. Human resource management, 
on the other hand, has evolved from focusing on local manpower pool to taking advantage of the overseas labour market. Nonetheless, whether they are locally or overseas-hired, employees are the ones facing the consequences of the unprecedented changes that the labour market is experiencing.

It has been found in this research that PD emanating from organizational structures and employer-employee relations are equally important. Personal characteristics play a smaller part in the understanding of the discrimination process within New Zealand organizations.

No longer can organizations be blind to the welfare of their employees. The role of organizational psychology is thereby critical in bridging organizations and employees so that they will be able to grow simultaneously in a globalized economy. Organizations must be aware of their role in ensuring that employees are not subjected to discriminatory policies and practices that have long-term and detrimental effects on personal and work outcomes of employees.

Correspondingly, all employees play an equally important role in maintaining a harmonious working environment through interactions that do not demean other employees because of dissimilar characteristics. More importantly, everybody needs to take responsibility to ensure that respect for all humanity shall be the foremost objective and not the usual objective of power and wealth. For the success of current globalized economies, I agree with the Dalai Lama's views, and I quote: ${ }^{8}$

"A new way of thinking has become the necessary condition for responsible living and acting. If we maintain obsolete values and beliefs, a fragmented

\footnotetext{
${ }^{8}$ Head of the Dge-lugs-pa order of Tibetan Buddhists, 1989 Nobel Peace Prize, b.1935.
} 
consciousness and self-centered spirit, we will continue to hold onto outdated goals and behaviors. Such an attitude by a large number of people would block the entire transition to an interdependent yet peaceful and cooperative global society." 


\section{References}

References marked with an asterisk (*) indicate studies included in the metaanalysis.

Aarons, G. A., \& Sawitzky, A. C. (2006). Organizational Climate Partially Mediates the Effect of Culture on Work Attitudes and Staff Turnover in Mental Health Services. Administration and Policy in Mental Health and Mental Health Services Research, 33(3), 289-301.

Alderfer, C. P., Alderfer, C. J., Tucker, L., \& Tucker, R. (1980). Diagnosing race relations in management. Journal of Applied Behavioral Science, $16,135-166$.

Allen, R. E., \& Keaveny, T. J. (1985). Factors Differentiating Grievants and Nongrievants. Human Relations, 38 (6), 519-534.

Allison, K. W. (1998). Stress and oppressed social category membership. In J. Swim, \& C. Stangor (Eds.), Prejudice: The target's perspective (pp. 145-170). San Diego, CA: Academic Press.

American Psychological Association (2001). Publication manual of the American Psychological Association (5 $5^{\text {th }}$ ed.). Washington, D.C.: American Psychological Association.

Araujo, B. Y., \& Borrell, L. N. (2006). Understanding the link between discrimination, mental health outcomes, and life chances among Latinos. Hispanic Journal of Behavioral Sciences, 28, 245-266.

Arrindell, W. A., Heesink, J., \& Feij, J. A. (1999). The satisfaction with life scale (SWLS): Appraisal with 1700 healthy young adults in The Neatherlands. Personality and Individual Differences, 26, 815-826.

Arrindell, W. A., Meeuwesen, L., \& Huyse, F. J. (1991). The Satisfaction with Life Scale (SWLS): Psychometric properties in a non-psychiatric medical outpatient sample. Personality and Individual Differences, 12, 117-123.

Arvey, R. D., Azevedo, R. E., Ostgarrd, D. J., \& Raghuram, S. (1996). The implications of a diverse labor market on human resource planning. In E. E. Kossek \& S. A. Lobel (Eds.), Managing diversity: Human resource 
strategies for transforming the workplace (pp. 51-73). Cambridge, MA: Blackwell.

Ashenfelter, O. \& Rees (Eds.) (1971). Discrimination in labor markets. A paper presented at the Conference on Discrimination in Labor Markets, Princeton University, New Jersey.

Australasian Research Management Society (2004). Beyond brain drain: Mobility, competitiveness and scientific excellence. A paper presented at the Regional workshop in the University of Queensland, Brisbane, Australia.

Aycan, Z. (2000). Cross-cultural industrial and organizational psychology: Contributions, past developments and future directions. Journal of Cross-Cultural Psychology, 31(1), 110-128.

Aydemir A., \& Skuterud, M. (2005). Explaining the deteriorating entry earnings of Canada's immigration cohorts: 1966-2000. Canadian Journal of Economics, 38, 641-671.

Badgett, M. V. L. (1995). The wage effects of sexual orientation discrimination. Industrial \& Labor Relations Review, 48(4), 726-739.

Baker M., \& Benjamin, D. (1997) Ethnicity, foreign birth and earnings: a Canada/U.S. comparison. In M.G. Abbott, C.M. Beach, \& R.P. Chaykowski (Eds.), Transition and structural change in the North American labour market( pp 281-313). Queen's University, Kingston: John Deutsch Institute and Industrial Relations Centre.

Balser, D.B. (2000). Perceptions of on-the-job discrimination and employees with disabilities. Employee Responsibilities and Rights Journal, 12(4), 179-197.

Balser, D. B. (2002). Agency in organizational inequality: Organizational behaviour and individual perceptions of discrimination. Work and Occupations, 29, 137-165.

Banerjee, R. (2008). An examination of factors affecting perceptions of workplace discrimination. Journal of Labor Research, 29, 380-401.

Barack, M. E. M., Cherin, D. A., \& Berkman, S. (1998). Organizational and personal dimensions in diversity climate: Ethnic and gender differences in employee perceptions. Journal of Applied Behavioral Science, 34 (1), 82-104. 
Baris, G., Ferreira, M. C., Assmar, E. M. L., Omar, A., Fischer, R., Huyhn, C. C., Dalyan, F., \& Hassan, A. (2005). Organizational practices from a global perspective. Paper presented at the 30th Interamerican Psychology Congress, Buenos Aires, Argentina.

Baron, J. N., \& Pfeffer, J. (1994). The social psychology of organizations and inequality. Social Psychology Quarterly, 57(3), 190-209.

* Barry, D. T., \& Grilo, C. M. (2003). Cultural, self-esteem, and demographic correlates of perception of personal and group discrimination among East Asian immigrants. American Journal of Orthopsychiatry, 7(2), 223229.

Basnayake A (1999). Employment experiences of Sri Lankan Migrants in New Zealand. New Zealand: Equal Employment Opportunities Trust.

Bobo, L., \& Kluegel, J. R. (1993). Opposition to race-targeting: Self-interest, stratification ideology, or racial attitudes? American Sociological Review, 58, 443-464.

Beatty, C. A. (1996). The stress of managerial and professional women: Is the price too high? Journal of Organizational Behavior, 17(3), 233-251.

Bedford, R., Ho, E., \& Lidgard, J. (2001). Immigration policy and New Zealands' development into the $21^{\text {st }}$ century: Review and speculation. In Y. Hayase, \& C.-L. Tsay (Eds.), Proceedings of the international workshop on international migration and structural change in the APEC member economies. Chiba, Japan: Institute of Developing Economies, JETRO.

Begg, C. B. (1994). Publication bias. In H. Cooper \& L. V. Hedges (Eds.), The Handbook of research synthesis (pp. 399-409). New York: Russell Sage Foundation.

Bell, M. P., Harrison, D. A., \& Mclaughlin, M. E. (1997). Asian American attitudes toward affirmative action in employment . The Journal of Applied Behavioral Science, 33 (3), 356-377.

Bennington, L., \& Wein, R. (2000). Focus on Australia: Anti-discrimination legislation in Australia: Fair, effective, efficient or irrelevant. International Journal of Manpower, 21, 21-33. 
Bergman, B. (2003). The validation of the women workplace culture questionnaire: Gender-related stress and health for Swedish working women. Sex Roles, 49(5/6), 287-297.

Bergman, B. \& Hallberg, L. R.-M. (2002). Women in a male-dominated industry: Factor analyses of a woman workplace culture questionnaire based on a grounded theory model. Sex Roles, 46, 305-316.

Berry, J.W. (1990). Psychology of acculturation. In J. Berman (Ed.), Crosscultural perspectives. Nebraska Symposium on motivation (Vol. 37, pp. 201-234). Lincoln: University of Nebraska Press.

Berry, J. W. (2006). Stress perspectives in acculturation. In D. L. Sam \& J. W. Berry (Eds.), The Cambridge handbook of acculturation psychology (pp. 43-57). Cambridge, UK: Cambridge University Press.

Berry, J. W., Phinney, J., Sam, D. L., \& Vedder, P. (Eds). (2006). Immigrant youth in cultural transition: acculturation and adaptation across national contexts. Mahwah, NJ: Lawrence Erlbaum Associates.

Bies, R. J., \& Moag, J. F. (1986). Interactional justice: Communication criteria of fairness. In R. J. Lewicki, B. H. Sheppard, \& M. H. Bazerman (Eds.), Research on negotiations in organizations (Vol. 1, pp. 43-55). Greenwich, CT: JAI Press.

Birzer, M. L., \& Ellis, R. B. (2006). Debunking the myth that all is well in the home of Brown v. Topeka Board of Education: A study of perceived discrimination. Journal of Black Studies, 36, 793-814.

Blackaby, D., Leslie, D., Murphy, P. \& O’Leary, N. (1998). The ethnic wage gap and employment differentials in the 1990s: evidence from Britain. Economic Letters, 58, 97-103.

Blackaby, D., Leslie, D., Murphy, P. \& O’Leary, N. (1999). Unemployment among Britain's ethnic minorities. The Manchester School, 67 (1), 1-20.

Blackaby, D.H., Leslie, D.G., Murphy, P.D., \& O'Leary, N.C. (2005). Born in Britain: How are native ethnic minorities faring in the British labour market? Economics Letters, 88, 370-375.

Bloom, D. E., Grenier, G., Gunderson, M. (1995). The changing labour marke position of canadian immigrants. Canadian Journal of Economics, 28, 987-1005. 
Bluedorn, A. C. (1982). A unified model of turnover from organization. Human Relations, 1(2), 135-153.

Bobo, L. D. \& Fax, C. (2003). Race, racism, and discrimination: Bridging problems, methods and theory in social psychology research. Social Psychology Quarterly, 66 (4), 319-332.

* Bourguignon, D., Seron, E., Yzerbyt, V., \& Herman, G. (2006). Perceived group and personal discrimination: Differential effects on personal selfesteem. European Journal of Social Psychology, 36, 773-789.

Bowman, P. J. (1991). Worklife. In J. S. Jackson (Ed.), Life in black America (pp.124-155). Thousand Oaks,CA: Sage Publications, Inc.

Boyatzis, R. E. (1998). Transforming qualitative information: Thematic analysis and code development. Thousand Oaks: Sage.

Branscombe, N. R., Schmitt, M. T., \& Harvey, R. D. (1999). Perceiving pervasive discrimination among African Americans: Implications for group identification and well-being. Journal of Personality and Social Psychology, 77, 135-149.

Braun, V., \& Clarke, V. (2006). Using thematic analysis in Psychology. Qualitative Research in Psychology, 3, 77-101.

Brief, A.P., Dietz, J., Cohen, R.R., Pugh, S.D., \& Vaslow, J.B. (2000). Just doing business: Modern racism and obedience to authority as explanations for employment discrimination. Organizational Behavior and Human Decision Processes, 81(1), 72-97.

* Brody, G. H., Chen, Y., Murry, V. M. Simons, R. L., Ge, X., Gibbons, F. X., Gerrard, M., \& Cutrona, C. E. (2006). Perceived discrimination and the adjustment of African American youths: A five-year longitudinal analysis with contextual moderation effects. Child Development, 77(5), 1170-1189.

Brosnan, P. (1988, Dec). Pacific island people: Migrant and minority workers in the New Zealand labour market. Paper prepared for Migrant Workers, Information and Human Rights Conference, Melbourne, Australia.

Brown, H. A., \& Ford, D. L. (1977). An exploratory analysis of discrimination in the employment of black MBA graduates. Journal of Applied Psychology, 62(1), 50-56. 
Brown, T. (2001). Measuring self-perceived racial and ethnic discrimination in social surveys. Sociological Spectrum, 21, 377-392.

Butcher, A., Spoonley, P., \& Trlin, A. (September 11, 2006). Being accepted: The experience of discrimination and social exclusion by immigrants and refugees in New Zealand. Massey University. Press release. Retrieved 2006-09-17.

Butterfield, S. P. (2004). Challenging American conceptions of race and ethnicity: Second generation west Indian immigrants. International Journal of Sociology and Social Policy, 24 (7/8), 75-102.

Callister, P. (2004). Seeking an ethnic identity: Is "New Zealander" a valid ethnic category? New Zealand Population Review, 30, 5-22.

Canales, M. (1997). Narrative interaction: Creating a space for therapeutic communication. Issues in Mental Health Nursing, 18, 477-494.

Cardo, L. M. (1994). Development of an instrument measuring valence of ethnicity and perceptions of discrimination. Journal of Multicultural Counseling and Development, 22, 49-59.

Cargile, A.C. (1997). Attitudes toward Chinese-accented speech: An investigation in two contexts. Journal of Language and Social Psychology, 16(4), 434-443.

Carnevale, A. P., \& Stone, S. C. (1995). Diversity beyond the golden rule. Training and development, 48, 22-37.

* Cassidy, C., O’Connor, R. C., Hower, C., \& Warden, D. (2005). Perceived discrimination among ethnic minority young people: The role of psychological variables. Journal of Applied Social Psychology, 35(6), 1246-1265.

Cervantes, R. C., Padilla, A. M., \& Salgado de Synder, N. (1991). The Hispanic Stress Inventory: A culturally relevant approach to psychosocial assessment. Psychological Assessment, 3, 438-447.

Chan, S., \& Stevens, A. H. (2001). Job loss and employment patterns of older workers. Journal of Labor Economics, 19(2), 484-521.

Chang. E. (1999). Career commitment as a complex moderator of organizational commitment and turnover intention. Human Relations, 52(19), 127-178. 
Chen, X. P., Hui, C., \& Sego, D. J. (1998). The role of organizational citizenship behavior in turnover: Conceptualization and preliminary tests of key hypotheses. Journal of Applied Psychology, 83 (6), 922-931.

Chen, Z. X., \& Francesco, A. M. (2000). Employee demography, organizational commitment and turn-over intentions in China: Do Cultural Differences Matter? Human Relations, 53 (6), 869-887.

Cheung, F., \& Dobkin de Rios, M. (1982). Recent trends in the study of the mental health of Chinese immigrants to the United States. Research in Race and Ethnic Relations, 3, 145-163.

Cheung, G.W., \& Rensfold, R.B. (2002). Evaluating goodness-of-fit indexes for testing measurement invariance. Structural Equation Modeling, 9, 233-255.

Chojnacki, J. T., \& Gelberg, S. (1994). Toward a conceptualization of career counseling with gay/lesbian/bisexual persons. Journal of Career Development, 21, 3-10.

Chung, Y. B. (2001). Work discrimination and coping strategies: Conceptual frameworks for counseling lesbians, gay and bisexual clients. The Career Development Quarterly, 50(1), 33-44.

Clark, R., Anderson, N. B., Clark, V. R., \& Williams, D. R. (1999). Racism as a stressor for African Americans: A biopsychosocial model. American Psychologist, 54, 805-816.

Cleaveland, J. N., \& Shore, L., M. (1992). Self- and supervisory perspectives on age and work attitudes and performance. Journal of Applied Psychology, 77, 469-484.

Coates, K. \& Carr, S. C. (2005) Skilled immigrants and selection bias: A theory based field study from New Zealand. International Journal of Intercultural Relations, 29(5), 577-599.

Cohen, A. (1993). Organizational commitment and turn-over. A metaanalysis. Academy of Management Journal, 36, 1140-1157.

Cohen, R. (Ed.). (1996). Theories of migration. Vermont, US: Edward Elgar Publishing Company.

Cole, S. W., Kemeny, M. E., Taylor, S. E., \& Visscher, B. R. (1996). Elevated physical health risk among gay men who conceal their homosexuality identity. Health Psychology, 15, 243-251.. 
Collado-Proctor, S. M. (1999). The perceived racism scale for Latina/os: A multidimensional assessment of the experience of racism among Latino/as. Unpublished doctoral dissertation. Durham, NC: Duke University.

Colquitt, J.A. (2001). On the dimensionality of organizational justice: A construct validation of a measure. Journal of Applied Psychology, 86, 386400.

Colquitt, J. A., Colon, D. E., Wesson, M. J., Porter, C. O. L. H., \& Ng, K. Y. (2001). Justice at the millennium: A meta-analytic review of 25 years of organizational justice research. Journal of Applied Psychology, 86 (3), 425-445.

* Combs, D. R., Penn, D. L., Cassisi, J., Michael, C., Wood, T., Wanner, J., \& Adams, S. (2006). Perceived racism as a predictor of paranoia among African Americans. Journal of Black Psychology, 32(1), 87-104.

* Constantine, M. G., Wallace, B. C., \& Kindaichi, M. M. (2005). Examining contextual factors in the career decision status of African American adolescents. Journal of Career Assessment, 3(3), 307-319.

Coombs, A. A., \& King, R. K. (2005). Workplace discrimination: experience of practicing physicians. Journal of the National Medical Association, 97 (4), 467-477.

Cortina, J. M. (1993). What is coefficient alpha? An examination of theory and applications. Journal of Applied Psychology, 78(1), 98-104.

Costa, P.T. \& McCrae, R.R. (1980). Still stable after all these years: personality as a key to some issues in adulthood and old age. In P. B. Baltes \& O. G. Brim, (Eds.). Life span development and behaviour, (3rd. ed., pp. 65-102). New York, NY: Academic Press.

Cox, T., Jr. (1994). Cultural diversity in organizations: Theory, research and practice. San Francisco, CA: Berrett-Koehler Publishers, Inc.

Cox, T., Jr. (2001). Creating the multicultural organization: A strategy of capturing the power of diversity. San Francisco, CA: Jossey-Bass.

Cox, T., Lobel S., \& McLeod, P. (1991). Effects of ethnic group cultural differences on cooperative and competitive behavior on a group task. Academy of Management Journal, 34, 827-847. 
Cox, T., \& Nkomo, S. M. (1992). Candidate age as a factor in promotability ratings. Public Personnel Management, 21, 197-210.

Cox, T., \& Nkomo, S. (1993). Race and ethnicity. In R. T. Golembiewski (Ed.), Handbook of Organizational Behavior. New York: Marcel Dekker.

Crocker, J., \& Major, B. (1989). Social stigma and self-esteem: The selfprotective properties of stigma. Psychological Review, 96(4), 608-630.

Crocker, J., Major, J., \& Steele, C. (1998). Social stigma. In D. Gilbert, S. T. Fiske, \& G. Lindsey (Eds.), Handbook of social psychology (4th ed., pp. 504-553). Boston, MA: McGraw-Hill.

Crocker, J., \& Quinn, D (1998). Racism and self-esteem. In J. L. Eberhardt \& S. T. Fiske (Eds.), Confronting racism: The problem and the response (pp. 169-187). Thousand Oaks, CA: Sage.

Crocker, J., Voelkl, K., Testa, M., \& Major, B. (1991). Social stigma: The affective consequences of attributional ambiguity. Journal of Personality and Social Psychology, 60, 218-228.

Cropanzano, R., \& Greenberg, J. (1997). Progress in organizational justice: Tunneling through the maze. In C. Cooper \& I. Robertson (Eds.), International review of industrial and organizational psychology (pp. 317-372). New York: Wiley.

Crosby, F. (1982). Relative deprivation and working women. New York: Oxford University Press.

Crosby, F. (1984). The denial of personal discrimination. The American Behavioral Scientist, 27(3), 371-386.

Cunningham, G.B., \& Sagas, M. (2004). Examining the main and interactive effects of deep and surface-level diversity on job satisfaction and organizational turnover intentions. Organizational Analysis, 12(3), 319341.

Darity, W. \&Goldsmith, A. H. (1993). Unemployment, social psychology, and unemployment hysteresis. Journal of Post Keynesian Economics 16, 55 72.

Davis, J. (1985). The logic of causal order. Beverly Hills, CA: Sage. 
De Castro, A. B., Gee, G. C., \& Takeuchi, D. T. (2008). Workplace discrimination and health among Filipinos in the United States. American Journal of Public Health, 98(3), 520-526.

De Silva, A. (1997). Wage discrimination against visible minority men in Canada. Canadian Business Economics, Summer, 25-42

* Deitch, E.A., Barsky, A., Butz, R.M., Chan, S., Brief, A. P., \& Bradley, J. C. (2003). Subtle yet significant: The existence and impact of everyday racial discrimination in the workplace. Human Relations, 56(11), 1229913009.

DeNavas-Walt, C., Proctor, B. D., Smith, J., U.S. Census Bureau (2007). Current population reports. Income, poverty and health insurance coverage in the United States: 2006 (pp. 60-233). Washington, DC: U.S. Government Printing Office.

Department of Labour (2005). Life in New Zealand: Settlement experiences of skilled migrants. Wellington: Department of Labour.

Dickerson, M. (1998, October). Women-owned businesses are fastest growing in U.S., report says. Los Angeles Times, p. C6.

Diener, E. (1984). Satisfaction with Life Scale (SWLS). Retrieved March 18, 2008 from http://www.psych.uiuc.edu/ ediener/hottopic/hottopic.html. (see also paper 1 on that page).

Diener, E., Emmons, R. A., Larsen, R. J., \& Griffin, A. (1985). The satisfaction with life scale. Journal of Personality Assessment, 49, 71-75.

DIMIA (Department of Immigration, Multicultural and Indigenous Affairs). (2003). Population Flows: Immigration Aspects 2007-2008 Edition. $\begin{array}{lllll}\text { Retrieved } & \text { April } & 20, & 2009\end{array}$ http://www.immi.gov.au/media/publications/statistics/popflows200708/PopFlows 09_chp6.pdf.

Dion, K.L. (1989). Ethnicity and perceived discrimination: a comparative survey of six ethnic groups in Toronto. Paper presented at the 10th Biennial Conference of the Canadian Ethnic Studies Association, Calgary, Alberta.

Dion, K. L., \& Kawakami, K. (1996). Ethnicity and perceived discrimination in Toronto: Another look at the personal/group discrimination discrepancy. Canadian Journal of Behavioral Science, 28, 203-213. 
Dipboye, R. L., \& Collela, A. (2005). Discrimination at work: The psychological and organizational bases. New Jersey: Lawrence Erlbaum Associates, Inc.

Donath, S. (2001). The validity of the 12-item General Health Questionnaire in Australia: A comparison between three scoring methods. Australian and New Zealand Journal of Psychiatry, 35, 231-235.

Dovidio, J. F., \& Gaertner, S. L. (Eds.). (1986). Prejudice, discrimination and racism. San Diego, CA: Academic Press.

Dovidio, J. F., \& Gaertner, S. L. (1993). Stereotypes and evaluative intergroup bias. In D. M. Mackie \& D. L. Hamilton (Eds.), Affect, cognition, and stereotyping: Interactive processes in group perception (pp. 167-193). San Diego, CA: Academic Press.

Dovidio, J. G., Gaertner, S. L., Niemann, Y. F., \& Snider, K. (2001). Racial, ethnic, and cultural differences in responding to distinctiveness and discrimination on campus: Stigma and common group identity. Journal of Social Issues, 57, 167-188.

Dovidio, J. F., \& Gaertner, S. L., \& Validzic, A. (1998). Intergroup bias: Status differentiation, and a common in-group identity. Journal of Personality and Social Psychology, 75, 109-120.

Dovidio, J. F., \& Hebl, M. R. (2005). Discrimination at the level of the individual: Cognitive and affective factors. In R. L Dipboye \& A. Colella (Eds), Discrimination at work: The psychological and organizational bases (pp.11-35). New Jersey: Lawrence Erlbaum Associates, Inc.

Dunham, R. B., Grube, J. A., \& Castaneda, M. B. (1994). Organizational commitment: The utility of an Integrative definition. Journal of Applied Psychology, 79, 370-380.

Dymski, G. A. (2001). Is discrimination disappearing? Residential credit market evidence, 1992-98. International Journal of Social Economics, 28, 1025-1045.

Eagly, A.H. \& Chaiken, S. (1993). The Psychology of Attitudes. Harcourt College Publishers. 
Earle, H. A. (2003). Building a workplace of choice: Using the work environment to attract and retain top talent. Journal of Facilities Management, 2, 244-257.

Eisenberger, R., Fasolo, P., \& Davis-LaMastro, V. (1990). Perceived organizational support and employee diligence, commitment, and innovation. Journal of Applied Psychology, 75 (1), 51-59.

Ellemers, N. (1993). The influence on socio-structural variables on identity management strategies. In W. Stroebe \& M. Hewstone (Eds.), European Review of Social Psychology (Vol. 4, pp. 27-58). New York: Wiley.

Ellemers, N., Wilke, H., \& van Knippenberg, A. (1993). Effects of the legitimacy of low group or individual status on individual and collective identity enhancement strategies. Journal of Personality and Social Psychology, 64, 766-778.

Elmslie, B., \& Sedo, S. (1996). Discrimination, social psychology, and hysteresis in the labour market. Journal of Economic Psychology, 17, 465-478.

* Ensher, E. A., Grant-Vallone, E. J., \& Donaldson, S. I. (2001). Effects of perceived discrimination on job satisfaction, organizational commitment, organizational citizenship behaviour and grievances. Human Resource Development Quarterly, 12(1), 53-72.

Equal Employment Opportunity Commission. (2008). Charge statistics FY 1997 through FY 2007. Retrieved February 3, 2009, from http:// www.eeoc.gov/ stats/ charges.html.

Essed, P. (1991). Understanding everyday racism: An interdisciplinary theory. Thousand Oaks, CA: Sage.

* Evans, K. M. \& Herr, E. L. (1994). The influence of racial identity and the perception of discrimination on the career aspirations of African American men and women. Journal of Vocational Behavior, 44, 173184.

Eyou, M. L., Adair, V., \& Dixon, R. (2000). Cultural identity and psychological adjustment of adolescent Chinese immigrants in New Zealand. Journal of Adolescence, 23, 531-534.

Feagin, J. R., \& Eckberg, D. (1980). Prejudice and discrimination. Annual Review of Sociology, 6, 1-20. 
Feagin, J. R. \& Feagin, C. B. (1978). Discrimination American style: Institutional racism and sexism. Englewood Cliffs, NJ: Prentice Hall.

Feather. N.T. \& Rauter, K.A. (2004). Organizational citizenship cBehaviours in relation to job status, job insecurity, organizational commitment and identification, job satisfaction and work values. Journal of Occupational and Oranizational Psychology, 77: 81 - 94.

Federal Glass Ceiling Commission (1995). Good for business: Making full use of the nation's human capital. Washington, DC: Federal Glass Commission.

Fernandez, J. P. (1981). Racism and sexism in corporate life: Changing values in American business. Lexington, MA: Lexington Books.

Fernandez, J. P. (1991). Managing a diverse workforce: Regaining the competitive edge. Lexington, MA: Lexington Books.

Fernandez, J. P. (1999). Race, gender, and rhetoric: The true state of race and gender relations in corporate America. New York: McGraw-Hill.

Finch, B. K., Kolody, B., \& Vega, W. A. (2000). Perceived discrimination and depression among Mexican-origin adults in California. Journal of Health and Social Behaviour, 41(3), 295-313.

Fischer, R., Ferreira, M. C., Assmar, E. M.. L., Redford, P., \& Harb, C. (2005). Organizational behaviour across cultures: Theoretical and methodological issues for developing multi-level frameworks involving culture. International Journal of Cross-Cultural Management, 5, 27-48.

Fischer, R., \& Mansell, A. (2009). Commitment across cultures: A metaanalytical approach. Journal of International Business Studies, doi:10.1057/jibs.2009.14.

Firkin, P., McLaren, E., Spoonley, P., de Bruin, A., Dupuis, A., Perera, H., et al. (2002). Non-standard work: Alternative working arrangements amongst knowledge workers. Labour Market Dynamics Research Programme, Albany and Palmerston North: Massey University.

* Flores, L. Y., Navarro, R. L., Smith, J. L., \& Ploszaj, A. M. (2006). Testing a model of nontraditional career choice goals with Mexican American adolescent men. Journal of Career Assessment, 14(2), 214-234. 
Foley, S., Hang-Yue, N., \& Wong, A. (2005). Perceptions of discrimination and justice: Are there gender differences in outcomes? Group \& Organization Management, 30(4), 421-450.

* Foley, S., \& Kidder, D. L. (2002). Hispanic law students' perceptions of discrimination, justice and career prospect. Hispanic Journal of Behavioural Sciences, 24(1), 23-37.

* Foley, S., \& Kidder, D. L., \& Powell, G. N. (2002). The perceived glass ceiling and justice perceptions: An investigation of Hispanic Law Associates. Journal of Management, 28(4), 471-496.

Forret, M., \& Love, M. S. (2008). Employee justice perceptions and coworker relationships. Leadership \& Organization Development Journal, 29 (3), 248-260.

Foster, M. D. \& Matheson, K. (1995). Double relative deprivation: Combining the personal and political. Personality and Social Psychology Bulletin, $21,1167-1177$.

* Foster, M. D., Sloto., L., \& Ruby, R. (2006). Responding to discrimination as a function of meritocracy beliefs and personal experiences: Testing the model of shattered assumptions. Group Processes \& Intergroup Relations, 9(3), 401-411.

Frattaroli, J. (2006). Experimental disclosure and its moderators: A metaanalysis. Psychological Bulletin, 132(6), 823-865.

* Fried, Y., Levi, A., Billings, S., \& Browne, K. (2001). The relationship between political ideology and attitudes toward affirmative action among African-Americans: The moderating effect of racial discrimination in the workplace. Human Relations, 54(5), 561-584.

Friedman, R., \& Davidson, M. N. (1999). The Black-White gap in perceptions of discrimination: Its causes and consequences. In R. J. Bies, R. J. Lewicki, \& B. H. Sheppard (Eds.), Research in negotiation in Organizations (pp.203-228). Stamford, CT: JAI Press.

Frieze, I. H., Olson, J., E., \& Good, D. C. (1990). Perceived and actual discrimination in the salaries of male and female managers. Journal of Applied Social Psychology, 20, 46-47. 
Frisina, P. G., Borod, J. C., \& Lepore, S. J. (2004). A meta-analysis of the effects of written emotional disclosure on the health outcomes in clinical populations. Journal of Nervous and Mental Diseases, 192, 629-634.

Furnham, A., \& Bochner, S. (1990). Culture shock: Psychological reactions to unfamiliar environment. London, New York: Routledge.

Gaertner, S. L., \& Dovidio, D. F. (1986). The aversive form of racism. In J.F. Dovidio \& S. L. Gaertner (Eds). Prejudice, discrimination and racism (pp. 61-89). San Diego, CA: Academic Press.

Gallo, L. C., \& Matthews, K. A. (1999). Do negative emotions mediate the association between socioeconomic status and health? In N. E. Adler, M. Marmot, B. S. McEwen, \& J. Stewart (Eds.), Socioeconomic status and health in industrial nations: Social, psychological, and biological pathways (pp. 226-245). New York, US: New York Academy of Sciences.

Garry, P. (2006). Cultural whiplash: Unforseen consequences on the promotion of American crusade against racial discrimination. Nashville, TN: Cumberland House Publishing.

Gecas, V., \& Schwalbe, M. L. (1983). Beyond the looking-glass self: Social structure and efficacy based self-esteem. Social Psychology Quarterly, 46, 77-88.

Gee, G. C., Delva., J. \& Takeuchi, D. T. (2007). Relationship between selfreported unfair treatment and prescription medication use, elicit drug use, and alcohol dependence among Filipino Americans. American Journal of Public Health, 97 (5), 933-940.

Gee, G. C., Pavalko, E. K., \& Long, J. S. (2007). Age, cohort and perceive age discrimination: Using the life course to assess self-reported age discrimination. Social Forces, 86(1), 265-290.

Gelfand, M. J., Nishi, L. H., Raver, J. L., \& Schneider (2005). Discrimination in organizations: An organizational-level systems perspective. In R. L Dipboye \& A. Colella (Eds). Discrimination at work: The psychological and organizational bases (pp. 89-115). New Jersey: Lawrence Erlbaum Associates, Inc.

Ghosh, R. (1984). Education, gender and the immigrant experiences. In R. J. Samuda, J. W. Berry, and M. Laferrire (Eds.), Multiculturalism in 
Canada: Social and educational perspectives. Newton, MA: Allyn \& Bacon.

*Gibbons, F. X., \& Gerrard, M., Cleveland, M. J., Wills, T. A., \& Brody, G. (2004). Perceived discrimination and substance use in African American parents and their children: A panel study. Journal of Personality and Social Psychology, 86(4), 517-529.

Giles, D. C. (2002). Advanced research methods in psychology. NY: Routledge.

Giles, H., \& Reid, S. A. (2005). Ageism across the lifespan: Towards a selfcategorization model of ageing. Journal of Social Issues, 61 (2), 389404.

Glick, P. (2002). Sacrificial lambs dressed in wolves' clothing: Envious, ideology and the scapegoating of Jews. In L.S. Newman \& R. Erber (Eds.), Understanding Genocide: The social psychology of the holocaust (pp. 113-142). Oxford, U.K.; Oxford University Press.

Goldberg, C. B. (2003). Applicant reactions to the employment interview: A look at demographic similarity and social identity theory. Journal of Business Research, 56, 561-571.

Goldberg, D. (1972). The detection of psychiatric illness by questionnaire. Oxford, UK: Oxford University Press.

Goldston, B. K. (2008). The relationship between traits of organizational culture and job satisfaction within the healthcare setting. Dissertation Abstracts International: Section B: The Sciences and Engineering, 68(11-B), 76-96.

Goto, S. H., Gee, G. C., \& Takuchi, D. T. (2002). Strangers still? The experiences of discrimination among Chinese Americans. Journal of Community Psychology, 30(2), 211-224.

Gottfredson, L. S. (1992). Dilemmas in developing diversity program. In S. E. Jackson \& Associates (Eds.), Diversity in the workplace (pp. 279-305). New York:Guilford.

Gouveia, V. V., Milfont, T. L., da Fonseca, P. N., \& de Miranda Coelho, J. A. P. (2009). Life satisfaction in Brazil: Testing the psychometric properties of the Satisfaction with Life Scale (SWLS) in five Brazilian samples. Social Indices Research, 90, 267-277. 
* Greene, M. L, Way, N., \& Pahl, K. (2006). Trajectories of perceived adult and peer discrimination among Black, Latino and Asian American adolescents: Patterns and psychological correlates. Developmental Psychology, 42(2), 218-238.

Griffeth, R. W., Hom, P. W., \& Gaertner, S. (2000). A meta-analysis of antecedents and correlates of employee turn-over: Update, moderator tests, and research implications for the next millennium. Journal of Management, 26, 463-488.

Guadagnoli, E., \& Velicer, W. F. (1988). Relation of sample size to the stability of component patters. Psychological Bulletin, 103, 265-275.

Gutek, B. A., Cohen, A. G., \& Tsui, A. (1996). Reactions to perceived discrimination. Human Relations, 49, 687-704.

Hackett, R., Bycio, P., \& Hausdorf, P. (1991). Further assessment of a three component model of organizational commitment. Proceedings of the Academy of Management, 212-216.

Hafer, C. L., \& Olson, J. M. (1993). Beliefs in a just world, discontent, and assertive actions by working women. Personality and Social Psychology Bulletin, 25, 139-148.

Hair, J. F., Anderson, R. E., Tatham, R. L., \& Black, W. C. (1998). Multivariate data analysis $\left(5^{\text {th }}\right.$ ed.). New Jersey: Prentice-Hall International.

Hair, J. F., Jr., Bush, R. P., \& Ortinau, D. J. (2006). Marketing research: Within a changing information environment ( $3^{\text {rd }}$ ed.). New York: McGraw-Hill/Irwin.

* Hall, S. P., \& Carter, R. T. (2006). The relationship between racial identity, ethnic identity, and perceptions of racial discrimination in an AfroCarribean descent sample. Journal of Black Psychology, 32(2), 155-175.

Halpert, J. A., Wilson, M. L., \& Hickman, J. L. (1993). Pregnancy as a source of bias in performance appraisal. Journal of Organization Behaviour, 14, 649-663.

Harrell, J. P., Hall, S., \& Taliaferro, J. (2003). Physiological responses to racism and discrimination: An assessment of the evidence. American Journal of Public Health, 93, 243-248. 
Harrison, D. A., Price, K. H., \& Bell, M. P. (1998). Beyond relational demography: Time and the effects of surface-and deep-level diversity on work group cohesion. Academy of Management Journal, 41 (1), 96-107.

Hawkins, J. D., Catalano, R. F., \& Miller, J. Y. (1992). Risk and protective factors for alcohol and other drug problems in adolescence and early adulthood: Implications for substance use prevention. Psychological Bulletin, 112, 64-105.

Healy, M.C., Lehman, M., \& McDaniel., M. A. (1995). Age and voluntary turnover: A quantitive review. Personnel Psychology, 48, 335-345.

Hebl, M. R., Foster, J. B., Mannix, L. M., \& Dovidio, J. F. (2002). Formal and interpersonal discrimination. A field study of bias towards homosexual applicants. Personality and Social Psychology Bulletin, 28, 815-825.

Hedges, L. V. (1994). Fixed effects model. In H. Cooper \& L. V. Hedges (Eds.), The handbook of research synthesis (pp. 285-300). New York: Russell Sage Foundation.

Herring, C., Thomas, M. E., Durr, M., \& Horton, H. D. (1998). Does race matter? The determinants and consequences of self-reports of discrimination victimization. Race and Society, 1(2), 109-123.

Hicks-Clarke, D., \& Iles, P. (2000). Climate for diversity and its effects on career and organizational attitudes and perceptions. Personnel Review, 29, 324-346.

Hirsch, B. T. \& Macpherson, D. A., \& Hardy, M. (2000). Occupational age structure and access for older workers. Industrial and Labor Relations Review, 53 (3), 401-18.

Hirsh, B.T., \& Schumacher, E. J. (1992). Labor earnings, discrimination and the racial composition of jobs. Journal of Human Resources, 27(4), 602628.

Ho, E. S. (1995). Chinese or New Zealander? Differential paths of adaptation of Hong Kong Chinese adolescent immigrant in New Zealand. New Zealand Population Review, 21, 27-50.

Ho, E. S., Lidgard, J., Bedford, R., \& Spoonley, P. (1997). East Asian migrants in New Zealand: Adaptation and employment. In A. D. Trlin \& P. Spoonley (Eds.), New Zealand and International Migration: A Digest 
and Bibliography No. 3. Palmerston North and Albany: Massey University.

Hofstede, G. (1997). Cultures and organizations: Software of the mind. US: McGraw-Hill Companies, Inc.

Hofstede, G., \& McCrae, R. R. (2004). Personality and culture revisited: Linking traits and dimensions of culture. Cross-cultural Research, 38(1), $52-58$.

Hollifield, S. T. (2006). An examination of teacher job satisfaction, workrelated stress and organizational culture in three school districts. Dissertation Abstracts International Section, 66 (11-A), 3877.

Holton, E.F. III \& Russell, C.J. (1999). Organizational entry and exit: An exploratory and longitudinal examination of elderly careers, Human Performance, 12(3/4), 311-342.

Huddy, L. (2001). From social to political identity: A critical examination of Social Identity Theory. Political Psychology, 22 (1), 127-156.

Hughes, A. O., Fenton, S., Hine, C. E., Pilgrim, S., \& Tibbs, N. (1995). Strategies for sampling black and ethnic minority populations. Journal of Public Health and Medicine, 17, 187-192.

Hum, D., \& Simpson, W. (1999). Wage opportunities for visible minorities in Canada. Canadian Public Policy, 25, 379-394.

Human Rights Commission (2000). Know your rights: The Human Rights Act and best business practice. Auckland, New Zealand: Human Rights Commission.

Human Rights Commission (2002). Report of the Human Rights Commission for the year ended 30 June 2002. Retrieved April 19, 2007, from http://www.hrc.co.nz/hrc new/hrc/cms/files/documents/12-Jun2005_20-39-15_Annual_Report_2002.pdf.

Human Rights Commission (2007). Annual Report 2007. Retrieved March 19, 2008, from http://www.hrc.co.nz/hrc new/hrc/cms/files/documents/19Nov-2007 11-42-32_HRC_ANNUAL_REPORT_07.pdf.

Hunter, J. E., \& Schmidt, F. L. (2004). Methods of meta-analysis: Correcting error and bias in research findings (2nd ed.). CA, Thousand Oaks: Sage Publications, Inc. 
Hurst, D. K. (1995). Crisis renewal: Meeting the challenges of organizational change. Boston: Harvard Business School Press.

Ibarra, H. (1993). Personal networks of women and minorities in management: A conceptual framework. Academy of Management Review, 18, 56-87.

International Labour Organization (2003). Time for equality at work: Global report under the follow-up to the ILO declaration on fundamental principles and rights at work. Geneva: International Labour Office.

International Labour Organization (2004). Combating discrimination against migrant and minority workers. Retrieved July 8, 2007 from http://www.ilo.org/public/english/protection/mirant/projects/discrim/.

International Organization for Migration. (2005). World migration 2005: Costs and benefits of international migration. Geneva: International Labour Office. Retrieved January 8, 2009; http://www.iom.int/jahia/Jahia/about-migration/facts-andfigures/regional-and-country-figures.

Ip, M. (2003). Chinese immigrants and transnationals in New Zealand: A fortress opened. In Ma, J. C. \& Cartier, C. (Eds.). (2003). The Chinese diaspora: Space, mobility and identity. US: Rowman \& Littlefield Publishers, Inc.

Jackson, D. J. R., Carr, S. C., Edwards, M., Thorn, K., Allfree, N., Hooks, J., \& Inkson, K. (2005). Exploring the dynamics of New Zealand's talent flow. New Zealand Journal of Psychology, 34(2), 110-116.

Jackson, S. E. (1992). Preview of the road to be travelled. In S. E. Jackson \& Associates (Eds.), Diversity in the Workplace (pp. 3-12). New York: Guildford.

Jackson, S. E., Brett, J. F., Sessa, V. I., Cooper, D. M., Julin, J. A., \& Peyronnin, K. (1991). Some differences make a difference: Individual dissimilarity and group heterogeneity as correlates of recruitment, promotions, and turn-over. Journal of Applied Psychology, 76, 675-689.

Jacques, R. (1997). The unbearable whiteness of being: Reflections of a pale, stale male. In P. Prasad, A. J. Mills, M. Elmes, \& A. Prasad (Eds.), Managing the organizational melting pot: Dilemmas of workplace diversity (pp. 80-106). Thousand Oaks, CA: Sage. 
James, K., Lovato, C., \& Cropanzano, R. (1994). Correlational and knowgroup comparison validation of a workplace prejudice/discrimination inventory. Journal of Applied Social Psychology, 24, 1573-1592.

Janoff-Bulman, R. (1992). Happystance. A review of subjective well-being: An interdisciplinary perspective. Contemporary Psychology, 37, 162163.

* Jasinskaja-Lahti, I., \& Liebkind, K. (2001). Perceived discrimination and psychological adjustment among Russian-speaking immigrant adolescents in Finland. International Journal of Psychology, 36(3), 174185.

* Jasinskaja-Lahti, I., Liebkind, K., Perhoniemi, R. (2007). Perceived ethnic discrimination at work and well-being of immigrants in Finland: The moderating role of employment status and work-specific group-level control beliefs. International Journal of Intercultural Relations, 31, 223242.

Jayaratne, S. (1993). The antecedents, consequences, and correlates of job satisfaction. In R. T. Golembiewski (Ed.), Handbook of organizational behavior (pp. 111-140). New York: Marcel Dekker.

* Jefferson, S. D., \& Caldwell, R. (2002). An exploration of the relationship between racial identity attitudes and the perception of racial bias. Journal of Black Psychology, 28(2), 174-192.

Jenkins, R. (1986). Racism and recruitment: Managers, organisations, and equal opportunity in the labour market. Cambridge: Cambridge University Press.

Jetten, J., Branscombe, N. R., Schmitt, M. T., \& Spears, R. (2001). Rebels with a cause: Group identification as a response to perceived discrimination from the mainstream. Personality and Social Psychology Bulletin, 27 (9), 1204-1213.

Johnson, B. T., \& Boynton, M. H. (2008). Cumulating evidence about the social animal: Meta-analysis in social-personality psychology. Social and Personality Psychology Compass, 2(2), 817-841.

Johnson, R. W., \& Neumark, D. (1997). Age discrimination, job separations, and employment status of older workers: Evidence from self-reports. The Journal of Human Resources 32(4): 779-881. 
Johnson, W.R. and Jones-John son, G. (1992) “Differential predictors of union and company commitment: Parallel and divergent models. Psychology, 29, 1-12.

Jost, J. T., \& Elsbach, K. D. (2001). How status and power differences erode personal and social identities at work: A system justification critique of organizational application of social identity theory. In M. A. Hogg \& D. J. Terry (Eds), Social identity processes in organizational contexts ( pp 181-196). Philadelphia, PA: Psychology Press.

Judge, T. A., Parker, S. H., Colbert, A. E., Heller, D., \& Elies, R. (2001). Job satisfaction: A cross-cultural review. In N. Anderson, D. S. Ones, H. K. Sinangil, \& C. Viswesvaran (Eds.), Handbook of Industrial Work and Organizational Psychology (Vol 2, pp. 25-52). Thousand Oaks: Sage Publications.

Kahn, W. A., \& Crosby, F. (1985). Discriminating between attitudes and discriminatory behaviors: Change and stasis. In L. Larwood, A. H. Stromberg, \& B. A. Gutek (Eds.), Women and work: An annual review (Vol. 1, pp. 215-238). Newbury Park, CA: Sage.

Kaiser, H. F. (1970). The second-generation little jiffy. Psychometrika, 35, 401-415.

Kalavar, J. M. (2001). Examining ageism: Do male and female college students differ? Educational Gerontology, 27 (6), 507-513.

Kanter, R. M. (1977). Men and women of the corporation. NY: Basic Books, Inc.

Karlsen, S., \& Nanoo, J. Y. (2002). Relation between racial discrimination, social class, and health among ethnic minority groups. American Journal of Public Health, 92, 624-631.

Katz, I., \& Hass, R. G. (1988). Racial ambivalence and value conflict: Correlational and priming studies of dual cognitive structures. Journal of Personality and Social Psychology, 40, 414-431.

Kessler, R. C., Mickelson, K. D., \& Williams, D. R. (1999). The prevalence, distribution, and mental health correlates of perceived discrimination in the United States. Journal of Health and Social Behavior, 40 (3), 208230. 
Kim, J. \& Mueller, C. W (1978). Introduction to factor analysis: What it is and how to do it. Quantitative Applications in the Social Sciences Series, (No. 13). Thousand Oaks, CA: Sage Publications.

Kinch, J. (1963). A formalized theory in self-concept. American Journal of Sociology, 68, 481-486.

Kinder, D. R. \& Sears, D. O. (1981). Prejudice and politics: Symbolic racism versus racial threats to the good life. Journal of Vocational Behavior, 63, 396-116.

Kirchmeyer, C. (2002). Gender differences in managerial careers: Yesterday, today and tomorrow. Journal of Business Ethnics, 37(2), 5-25.

Kling, K. C., Hyde, J. S., Showers, C. J., \& Buswell, B. N. (1999). Gender differences in self-esteem: A meta-analysis. Psychological Bulletin, 125, 470-500.

Ko, J., Price, J. L., \& Mueller, C. W. (1997). Assessment of Meyer and Allen's three component model of organizational commitment in South Korea. Journal of Applied Psychology, 82 (6), pp 961-973.

Kobrynowicz, D., \& Branscombe, N. R. (1997). Who considers themselves victims of discrimination? Psychology of Women Quarterly, 21, 347363.

Konovsky, M. A., \& Cropanzano, R. (1991). Perceived fairness of employee drug testing as a predictor of employee attitudes and job performance. Journal of Applied Psychology, 6, 698-707.

Konrad, A. M., \& Hartmann, L. (2001). Gender differences in attitudes toward affirmative action programs in Australia: Effects of beliefs, interests, and attitudes toward women. Sex Roles, 45, 415-432.

Konrad, A. M., \& Linnehan, F. (1999). Affirmative action: History, effects, and attitudes. In G.N. Powell (Ed.), Handbook of gender and work. (pp. 429-452). Thousand Oaks, CA: Sage.

* Konrad, A. M. \& Spitz, J. (2003). Explaining demographic differences in affirmative action attitudes. Journal of Applied Social Psychology, 33(8), 1618-1642.

Kravitz, D. A., Klineberg, S. L., Avery, D. R., Nguyen, A. K., Lund, C., \& Fu, E. J. (2000). Attitudes toward affirmative action: Correlations with 
demographic variables and with beliefs about targets, actions, and economic effects. Journal of Applied Social Psychology, 30, 1109-1136.

Krieger, N., \& Sidney, S. (1996). Racial discrimination and blood pressure: The CARDIA study of young black and white adults. American Journal of Public Health, 86, 1370-1378.

Lalonde, R. N. (1992). The dynamics of group differentiation in the face of defeat. Personality and Social Psychology, 18, 336-342.

Landrine, H., \& Klonoff, E. A. (1996). The schedule of racist events: A measure of racial discrimination and a study of its negative physical and mental health consequences. Journal of Black Psychology, 22, 144-168.

Lankau, M. J. \& Scandura, T. A. (2002). Mentoring and personal learning: Content, antecedents and outcomes. Academy of Management Journal, 45, 779-790.

Lawrence, B. S. (1984). Age grading: The implicit organizational timetable. Journal of Occupational Behavior, 13, 181-191.

Lawrence, B. S. (1990). At the cross roads: A multi-level explanation of individual attachment. Organization Science, 1, 65-85.

Lazarus R., \& Folkman, S. (1984). Stress, Appraisal, and Coping. NY: Springer Publishing Company.

* Lease, S. H. (2006). Factors predictive of the range of occupations considered by African American juniors and seniors in high school. Journal of Career Development, 32(4), 333-350.

* Lee, R. M. (2003). Do ethnic Identity and other-group orientation protect against discrimination for Asian Americans? Journal of Counselling Psychology, 50, 133-141.

Leeming, A., \& Baruch, Y. (1998). The MBA as a bridge over the troubled waters of discrimination. Women in Management Review, 13(3), 95-104.

Lester, R. A. (1980). Reasoning about discrimination: The analysis of professional and executive work in federal antibias programs. New Jersey: Princeton University Press.

* Levin, S., Sinclair, S., Veniegas, R. C., \& Taylor, P. L. (2002). Perceived discrimination in the context of multiple group memberships. Psychological Science, 13(6), 557-560. 
Levine, M., \& Leonard, R. (1984). Discrimination against lesbians in the workforce. Signs: Journal of Women in Culture and Society, 9(4), 700708.

Levitin, T., Quinn, R. P., \& Staines, G. L. (1971). Sex discrimination against the American working woman. The American Behavioral Scientist (pre1986), 15(2), 237-254.

Li, P. S. (2001). The market worth of immigrants' educational credentials. Canadian Public Policy, 27, 23-38.

Li, P. S. (2003). Initial earnings and catch-up capacity of immigrants. Canadian Public Policy, 29, 319-337.

Li, P. S. (2007). World migration in the age of globalization: Policy implications and challenges. Paper presented at the BRCSS New Settler Research Conference, Auckland, New Zealand.

Lipsey, M. W., \& Wilson, D. B. (1993). The efficacy of psychological, educational and behavioral treatment: Confirmation from meta-analysis. American Psychologist, 48, 1181-1209.

Lipsey, M. W., \& Wilson, D. B. (2001). Practical meta-analysis: Applied Social Research Methods Series. Volume 49. CA, Thousand Oaks: Sage Publications, Inc.

Locke, E. A. (1976). The nature and consequences of job satisfaction. In M. D. Dunnette (Ed.), Handbook of industrial and organizational psychology. Chicago: Rand-McNally.

Longhi, S., Nijamp, P., \& Poot, J. (2006). The fallacy of “Job Robbing”. Tinbergen Institute Discussion Paper. Netherlands: Tinbergen Institute. Retrieved on February 19, 2007 from http://www.tinbergen.nl.

Low, G. S., Cravens, D. W., Grant, K., Moncrief, W. C. (2001). Antecedents and Consequences of Salesperson Burnout. European Journal of Marketing, 35(5/6), 587-611.

Lundahl, M., \& Wadensjo, E. (1984). Unequal treatment: A study in the neoclassical theory of discrimination. Beckenham: Croom Helm.

Ma, J. C., \& Cartier, C. (Eds.). (2003). The Chinese diaspora: Space, mobility and identity. US: Rowman \& Littlefield Publishers, Inc.

Mace, K. A., Atkins, S., Fletcher, R., \& Carr, S. (2005). Immigrant job hunting, labour marke experiences, and feelings about occupational 
satisfaction in New Zealand: An exploratory study. New Zealand Journal of Psychology, 34 (2), 97-109.

Madden, J. (1985). The persistence of pay differentials: The economics of sex discrimination. In L. Larwood, A. H. Stromberg, \& B. A. Gutek (Eds.), Women and work: An annual review (vol. 1, pp. 76-112). Newbury Park, CA: Sage.

Mahroum, S. (2000). Highly skilled globetrotters: Mapping the international migration of human capital. $R$ \& D Management, 30(1), 23-32.

Major, B., Quinton, W. J., \& McCoy, S. K. (2002). Antecedents and consequences of attributions to discrimination: Theoretical and empirical advanes. In M. P. Zanna (Ed.), Advances in experimental social psychology (Vol. 34, pp. 251-329). San Diego: Academic Press.

* Malcarne, V. L., Chavira., D. A., Fernandez, S., \& Liu, P. (2006). The scale of ethnic experience: Development and psychometric properties. Journal of Personality Assessment, 86(2), 150-161.

Marger, M. N. (2000). Race and ethnic relations: American and global perspectives ( $5^{\text {th }}$ ed.). Belmont, CA: Wadsworth/Thomson Learning.

Marin, G. (1992). Issues in the measurement of acculturation among Hispanics. In K. F. Geisinger (Ed.), Psychological testing of Hispanics (pp. 11-30). Albany: State University of New York Press.

Marsella, A. J., \& Ring, E. (2003). Human migration and immigration: An overview. In L.L. Adler \& U. P. Gielen (Eds.), Migration: Immigration and emigration in international perspectives. CT: Greenwood Press.

Marsh, N. R., \& McDonald, W. F. (Eds.). (1983). The multi-cultural workforce in New Zealand and Australia. Auckland: University of Auckland.

Martin, P., Abella, M., \& Migley, E. (2004). Best practices to manage migration: The Philippines. International Migration Review, 38(4), $1544-1570$.

Martin, J. K., Tuch, S. A., \& Roman, P. M. (2003). Problem drinking patterns among African Americans: The impacts of reports of discrimination, perceptions of prejudice, and "risky" coping strategies. Journal of Health and Social Behavior, 44(3), 408-425. 
Massey, D. S., Durand, J., \& Malone, N. J. (2005). Principles of operation: Theories of international migration. In M. M. Suarez-Orozco, C. SuarezOrozco \& D. B. Qin (Eds.), The new immigration: An interdisciplinary reader. New York: Taylor and Francis Group.

Maydell-Stevens, E. (2004). Problems of psychological and sociocultural adaptation among Russian-speaking immigrants in New Zealand. Unpublished manuscript, Victoria University of Wellington, New Zealand.

Mays, V. M., Coleman, L. M., \& Jackson, J. S. (1996). Perceived race-based discrimination, employment status, and job stress in a national sample of Black women: Implications for health outcomes. Journal of Occupational Health Psychology, 1(3), 319-329.

McConahay, J. B. (1986). Modern racism, ambivalence, and the Modern Racism Scale. In J.F. Dovidio \& S.L. Gaernter (Eds.), Prejudice, discrimination and racism (pp. 91-125). San Diego, CA: Academic Press.

McCrae, R., \& Costa, P. (1995). Trait explanations in personality psychology. European Journal of Personality, 9, 231-252.

McDonald, R., Vechi, C., Bowman, J., \& Sanson-Fisher, R. (1996). Mental health status of a Latin American community in New South Wales. Australian and New Zealand Journal of Psychiatry, 30, 457-462.

McGee, G.W., \& Ford, R. C. (1987). Two (or more?) dimensions of organizational commitment: Reexamination of the affective and continuance commitment scales. Journal of Applied Psychology, 72, $638-642$.

McGoldrick, A. E. \& Arrowsmith, J. (1993). Recruitment advertising: Discrimination on the basis of age. Employee Relations, 15 (5), 54-65.

McGuire, W. J. (1985). Attitudes and attitude change. In G. Lindzey \& E. Aronson (Eds.), Handbook of social psychology (Vol. 2, pp. 233-346). New York: Random House.

McKinnon, M. (1996). Immigrants and citizens: New Zealanders and Asian immigration in historical context. Institute of Policy Studies, Victoria University of Wellington. Wellington: The Printing Press. 
McNeese-Smith, D.K. and Nazarey, M. (2001) “A nursing shortage: Building organizational commitment among nurses/practitioner application. Journal of Healthcare Management, 46(3), 173-187.

McNeilly, M. D., Anderson, N. B., Armstead, C. A., Clark, R., Corbet, M., Robinson, E. L., Pieper, C. F., \& Lepisto, E. M. (1996). The perceived racism scale: A multi-dimensional assessment of the experience of White racism among African-Americans. Ethnicity \& Disease, 6, 154-156.

McNemar, Q. (1960). At random: Sense and non-sense. American Psychologist, 15, 295-300.

McWhirter, E. H. (1997). Perceived barriers to education and career: Ethnic and gender differences. Journal of Vocational Behavior, 50, 124-140.

McWhirter, E. H., Hackett, G., \& Bandalos, D. L. (1998). A causal model of the educational plans and career expectations of Mexican American high school girls. Journal of Counseling Psychology, 45, 166-181.

Mena, F. J., Padilla, A. M., \& Maldonado, M. (1987), Acculturative stress and specific coping strategies among immigrant and later generation college students. Hispanic Journal of Behavioral Sciences, 9, 207-225.

Merriam, S. B. (1988). Case study research in education: A qualitative approach. San Francisco:Jossey-Bass.

Meyer, I. H. (2003). Prejudice, social stress, and mental health in lesbian, gay, and bisexual populations: Conceptual issues and research evidence. Psychological Bulletin, 129, 674-697.

Meyer, I. H. (2003). Prejudice, social stress, and mental health in lesbian, gay, and bisexual populations: Conceptual issues and research evidence. Psychological Bulletin, 129, 674-697.

Meyer, J. P. \& Allen, N. J. (1991). A three-component conceptualization of organizational commitment. Human Resource Management Review, 1(1), 61-89.

Meyer, J. P. \& Allen, N. J. (1997). Commitment in the workplace: Theory, research and application. Thousand Oaks: Sage Publications.

Meyer, J. P., Allen, N. J., \& Smith, C. A. (1993). Commitment to organizations and occupations: Extension and test of a three-component conceptualization. Journal of Applied Psychology, 78, 538-551. 
Meyer, J. P., Paunonen, S. V., Gellatly, I., R., Goffin, R. D., \& Jackson, D. N. (1989). Organizational commitment and job performance: It's the nature of commitment that counts. Journal of Applied Psychology, 74, 152-156.

Meyer, J. P., Stanley, D. J., Herscovitch L., \& Topolnytsky, L. (2002). Affective, continuance, and normative commitment to the organization: A meta-analysis of antecedents, correlates, and consequences. Journal of Vocational Behavior, 61, 20-52.

Milfont, T. L. (2007). Psychology of environmental attitudes: A cross-cultural study of their content and structure. Unpublished doctoral dissertation, University of Auckland, Auckland, New Zealand.

Miller, C. T., \& Kaiser, C. R. (2001). A theoretical perspective on coping with stigma. Journal of Social Issues, 57, 73-92.

Moghaddam, F. M., \& Taylor, D. M. (1987). The meaning of multiculturalism for visible minority immigrant women. Canadian Journal of Behavioural Science, 19, 122-139.

Moghaddam, F. M., Taylor, D. M., Ditto, D., Jacobs, K., \& Bianchi, E. (2002). Psychological distress and perceived discrimination: A study of women from India. International Journal of Intercultural Relations, 26, 381390.

Moorman, R. H. (1991). Relationship between organizational justice and organizational citizenship behaviors: Do fairness perceptions influence employee citizenship? Journal of Applied Psychology, 76, 845-855.

Moorman, R. H., Neihoff, B. P., \& Organ, D. W. (1993). Treating employees fairly and organizational citizenship behavior: Sorting the effects of job satisfaction, organizational commitment and procedural justice. Employee Responsibilities and Rights Journal, 6, 209-225.

* Moradi, B., \& Risco, C. (2006). Perceived discrimination experiences and mental health of Latina/o American Persons. Journal of Counselling Psychology, 53(4), 411-421.

Morrison, A. M., White, R. P., \& Van Velsor, E. (1987): Breaking the glass ceiling. Reading, MA: Addison-Wesley.

Mowday, R. T., Porter, L. W, \& Steers, R. M. (1982). Employeeorganizational linkages: The psychology of commitment, absenteeism, and turn-over. New York: Academic Press. 
Mueller, C. W., Finley, A., Iverson, R. D., \& Price, J. L. (1999). The effects of group racial composition on job satisfaction, organizational commitment, and career commitment: The case of teachers. Work and Occupations, 26, 187 - 219.

Mummendey, A., \& Schreiber, H. J. (1984). "Different" just means “better": Some observations and some hiden pathways to in-group favouritism. British Journal of Social Psychology, 23, 363-368.

Murphy, N. (2002). The Poll-tax in New Zealand. New Zealand Chinese Association, Office of Ethnic Affairs, Wellington.

Myers, D. G., \& Diener, E. (1997). The pursuit of happiness. Scientific American, 7, 40-43.

National Coordinating Committee for UDHR (1998). Drafting and adoption: The universal declaration of human right. Franklin and Eleanor Roosevelt Institute. Retrieved on July 2, 2008 from http://www.udhr.org/history/overview.htm.

Nelson, T. D. (2005). Ageism: Prejudice against our feared future self. Journal of Social Issues, 61 (2), 207-221.

New Asian and Migrant Health Research Centre (2004). New Asian and migrant health research centre established at Auckland University of Technology. Retrieved on August 4, 2005 from http:\|scoop.co.nz/stories/ED0408/S00064.htm.

New Zealand Department of Labour (2008). Migration trends 2006/2007. Wellington: Department of Labour.

New Zealand Immigration Service (2003). Skilled immigrants: Labour market experiences. Wellington: Department of Labour.

New Zealand Immigration Service (2009). English language requirements. Retrieved on February 10, 2009 from http://www.immigration.govt.nz/migrant/stream/work/skilledmigrant/can iapply/requirements/english/default.htm

New Zealand State Services Commission (2002). Annual report of the States Services Commission for the year ended 30 June 2002, including the annual report of the States Services Commissioner. Retrieved on August September 26 , 2007 from 
http://www.ssc.govt.nz/display/document.asp?NavID=118\&DocID=500 9

Ng, T. W. H., Eby, L. T., Sorensen, K. L., \& Feldman, D. C. (2005). Predictors of objective and subjective career success: A meta-analysis. Personnel Psychology, 58(2), 367-408.

Noh, S., Beiser, M., Kaspar, V., Hou, F., \& Rummens, J. (1999). Perceived racial discrimination, depression and coping: A study of Southeast Asian refugees in Canada. Journal of Health and Social Behavior, 40, 193-209.

Nunnally, J. C., \& Bernstein, I. H. (1994). Psychometric theory (3rd. ed.). New York: McGraw-Hill.

O’Boyle, E. J. (2001). Salary compression and inversion in the university workplace. International Journal of Social Economics, 28, 959-979.

Oliver, P. (2000). Employment for professional migrants to New Zealand: Barriers and opportunities. Wellington, New Zealand: Centre for Operational Research and Evaluation (Work and Income New Zealand).

* Oppedal, B., Roysamb, E., \& Sam, D. L. (2004). The effect of acculturation and social support on change in mental health among young migrants. International Journal of Behavioral Development, 28(6), 481-494.

Ornstein, M. (2000). Ethno-racial inequality in metropolitan Toronto: analysis of the 1996 census. York University, Toronto: Institute for Social Research.

O’Reilly, III, C., \& Chatman, J. (1986). Organizational commitment and psychological attachment: The effects of compliance, identification, and the internalization on pro-social behaviour. Journal of Applied Psychology, 71 (3): 492-499.

Palameta, B. (2004). Low income among immigrants and visible minorities. Perspectives on Labour and Income, 5, 12-17.

Patterson, G. R., DeBaryshe, B. D., \& Ramsey, E. (1989). A developmental perspective on antisocial behaviour. American Psychologist, 44, 329335.

Patton, M. Q. (1990). Qualitative evaluation and research methods ( $2^{\text {nd }} \mathrm{ed}$.) Thousand Oaks: Sage.

Pavalko, E. K., Mossakowski, K. N., \& Hamilton, V. J. (2003). Does perceived discrimination affect health? Longitudinal relationships 
between work discrimination and women's physical and emotional health Journal of Health and Social Behavior, 44 (1), 18-34.

Pendakur K., \& Pendakur, R. (1998) The colour of money: earnings differentials among ethnic groups in Canada. Canadian Journal of Economics, 31, 518-548.

Pernice, R., \& Brook, J. (1996). Refugees' and immigrants' mental health. Association of demographic and post-immigration factors. The Journal of Social Psychology, 136 (4), 511-519.

Pernice, R., Trlin, A., Henderson, A., \& North, N. (2000). Employment and mental health of three groups of immigrants to New Zealand. New Zealand Journal of Psychology, 29 (1), 24-29.

Petersen, L-E., and Dietz, J. (2005). Prejudice and enforcement of workforce homogeneity as explanations for employment discrimination. Journal of Applied Social Psychology, 35(1), 144-159.

Petraitis, J., Flay, B. R., \& Miller, T. Q. (1995). Reviewing theories of adolescent substance use: Organizing pieces of the puzzle. Psychological Bulletin, 117, 67-86.

Pettigrew, T. F. (2002). Summing up: Relative deprivation as a key social concept. In I. Walker \& H. S. Smith (Eds.), Relative deprivation: Specification, development and integration (pp. 351-373). New York: Cambriage University Press.

Phan, B., \& Kleiner, B. H (1999). The basics of employment discrimination. Equal Opportunities International, 18(8), 10-15.

Phelps, E. S. (1972). The statistical theory of racism and sexism. American Economic Review, 62(4), 659-661.

Phillips, J. (2008). History of immigration: Te Ara - the encyclopedia of New Zealand. Retrieved on April 15, 2008 from http://www.TeAra.govt.nz/ NewZealanders/NewZealandPeoples/HistoryOfImmigration/en.

Phinney, J. S. (1990). Ethnic identity in adolescents and adults: Review of research. Psychological Bulletin, 108, 499-514.

Phinney J. S., Berry, J. W., Sam, D. L., \& Vedder P., et al. (2006). Understanding immigrant youth: Conclusions and Implications. In J. W. Berry, J. S. Phinney, D. L. Sam, \& P. Vedder (Eds.), Immigrant youth cultural transition: Acculturation, identity and adaptation across 
national context (Chapter 9). New Jersey: Lawrence Erlbaum Associates.

* Phinney, J. S., Madden, T., \& Santos, L. J. (1998). Psychological variables as predictors of perceived ethnic discrimination among minority and immigrant adolescents. Journal of Applied Social Psychology, 28(11), 937-953.

Pinel, E. C. (1999). Stigma consciousness: The psychological legacy of social stereotypes. Journal of Personality and Social Psychology, 76(1), 114128.

Podsiadlowski, A (2002). Multicultural work groups in organizations: What factors are decisive for the success or failure of internationally composed work groups? Conditions for effective co-operation in the case of German companies doing business in Asia Pacific. A doctoral thesis. Unpublished Manuscript.

Podsiadlowski, A. (2006). FRST-Endreport: Facilitating Migrants' Entry and Integration into the Workplace, as part of Migrants' Settlement Research Project: Strangers in Town (led by $R$. Bedford), Contract\# UWWX203.Hamilton, New Zealand: University of Waikato.

Podsiadlowski, A., \& Ward, C. (forthcoming). Global Mobility and Bias in the Workplace. S. C. Carr (Ed.). The Psychology of Mobility in a Global Era.

Porter, L., Steers, R., Mowday, R., \& Boulian, P. \{1974). Organizational commitment., job satisfaction and turnover among psychiatric technicians. Journal of Applied Psvchology, 59, 603-609.

Pratto, F., Sidanius, J., \& Levin, S. (2006). Social dominace theory and the dynamics of intergroup relations: Taking stock and looking forward. European Reivew of Social Psychology, 17, 271-320.

Premeaux, S. F. (2001). Impact of applicant disability on selection: The role of disability, type, physical attractiveness and proximity. Journal of Business and Psychology, 16(2), 291-298.

Quinn, D. M., \& Crocker, J. (1999). When ideology hurts: Effects of feeling fat and the Protestant ethic on the psychological being of women. Journal of Personality and Social Psychology, 77, 402-414. 
Quinn, R. E., \& Spreitzer, G. M. (1991). The psychometrics of the competing values culture instrument and an analysis of the impact or organizational culture on quality of life. Research in Organizational Change and Development, 5, 115-142.

Ragins, B. R. (1999). Gender and mentoring relationships. A review and research agenda for the next agenda. In G. N. Powell (Ed.), Handbook of gender and work (pp. 347-370). Thousand Oaks: Sage.

Ragins, B. R., \& Cornwell, J. M. (2001): Pink triangles: antecedents and consequences of perceived workplace discrimination against gay and lesbian employees. Journal of Applied Psychology, 86, 1244-1261.

Ragins, B. R., \& Sundstrom, E. (1989). Gender and power in organizations: A longitudinal perspective. Pyschological Bulletin, 105, 51-88.

Rakow, L. F., Wackwitz, L. A. (1998). Communication of sexism. In M. L. Hecht (Ed.), Communicating prejudice (pp. 99-111). Thousand Oaks, CA: Sage.

Randall, C. S., Cropanzano, R., Bormann, C. A., \& Birjulin, A. (1999). Organizational politics and organizational support as predictors of work attitudes, job performance, and organizational citizenship behavior. Journal of Organizational Behavior, 20, 159-174.

Raudenbush, S. W. (1994). Random effects models. In H. Cooper \& L. V. Hedges (Eds.), The handbook of research synthesis (pp. 301-322). New York: Russell Sage Foundation.

Reichers, A. E. \& Schneider, B. (1990). Climate and culture: An evolution of constructs. In B. Schneider (Ed.), Organizational climate and culture (pp. 5-39). San Francisco: Jossey-Bass.

Reitz, J. G. (2001). Immigrant skill utilization in the Canadian labour market: implications of human capital research. Journal of International, Migration and Integration, 2, 347-378.

Reitz, J. G., \& Banerjee, R. (2007). Racial inequality, social cohesion, and policy issues in Canada. In T. J. Courchene, K. Banting \& W. Wuttunee (Eds.), Belonging? Diversity, recognition and shared citizenship in Canada (489-545). Montreal: Institute for Research on Public Policy.

Reskin, B. F. (1998). The realities of affirmative action in employment. Washington, DC: American Sociological Association. 
Reskin, B. F. (2000). The proximate causes of employment discrimination. Contemporary Sociology, 29(2), 319-328.

Riordan, C.M., Shaffer, B. S., \& Stewart, M. M. (2005). Relational demography within groups: Through the lens of discrimination. In R. L. Dipboye \& A. Colella (Eds.), Discrimination at work: Psychological and organizational bases. (pp. 37-61). New Jersey: Lawrence Earlbaum Associates, Inc.

Riordan, C. M., \& Shore, L. M. (1997). Demographic diversity and employment attitudes: An empirical examination of relational demography within work units. Journal of Applied Psychology, 82, 342358.

Rodin, M. J., Price, J. M., Bryson, J. B., \& Sanchez, F. J. (1990). Asymmetry in prejudice attribution. Journal of Experimental Social Psychology, 26, 481-504.

* Rollins, V. B., \& Valdez, J. N. (2006). Perceived racism and career selfefficacy in African American Adolescents. Journal of Black Psychology, 32(2), 176-198.

* Romero, A. J., \& Roberts, R. E. (1998). Perception of discrimination and ethnocultural variables in a diverse group of adolescents. Journal of Adolescents, 21, 641-656.

Romero, A. J., \& Roberts, R. E. (2003a). Stress within a bicultural context for adolescents of Mexican descent. Cultural Diversity and Ethnic Minority Psychology, 9(2), 171-184.

* Romero, A. J., \& Roberts, R. E. (2003b). The impact of multiple dimensions of ethnic identity on discrimination and adolescents' self-esteem. Journal of Applied Social Psychology, 33(11), 2288-2305.

Rosenthal, R. (1984). Meta-analytic procedures for social research, Sage: Beverly Hills.

Rosin, H. M., \& Korabik, K. (1991). Workplace variables, affective responses, and intention to leave among women managers. Journal of Occupational Psychology, 64, 317-330.

Ruggiero, K. M., \& Taylor, D. M. (1995). Coping with discrimination: How disadvantaged group members perceive the discrimination that confronts them. Journal of Personality and Social Psychology, 68(5), 826-838. 
Ruggiero, K. M., \& Taylor, D. M. (1997). Why minority group members perceive or do not perceive the discrimination that confronts them: The role of self-esteem and perceived control. Journal of Personality and Social Psychology, 72(2), 373-389.

Runciman, W. G. (1972). Relative deprivation and social justice: A study of attitudes to social inequality in twentieth-century England. Harmondsworth: Penguin.

Ryff, C. D. (1989). Happiness is everything, or is it? Explorations on the meaning of psychological well-being. Journal of Personality and Social Psychology, 57, 1069-1081.

* Sanchez, J. I., \& Brock, P. (1996). Outcomes of perceived discrimination among Hispanic employees: Is diversity management a luxury or a necessity? Academy of Management Journal, 39(3), 704-719.

Schneider, B. (1987). The people make the place. Personnel Psychology, 40, 437-453.

Schneider, B. (2000). The psychological life of organizations. In N. M. Ashkanasy, C. P. M. Wilderom \& M. F. Peterson (Eds.). Handbook of organizational culture and climate (pp. xvii-xxii). Thousand Oaks, CA: Sage.

Schneider, K.T., Swan, S. \& Fitzgerald, L.F. (1997). Job-related and psychological effects of sexual harassment in the workplace: Empirical evidence from two organizations. Journal of Applied Psychology, 82, 401-15.

Schittker, J. \& McLeod, J. D. (2005). The social psychology of health disparities. Annual Review of Sociology, 31, 75-103.

* Scott, L. D. Jr. (2003). The relation of racial identity and racial socialization to coping with discrimination among African American Adolescents. Journal of Black Studies, 33(4), 520-538.

* Scott, L. D. Jr., \& House, L. E. (2005). Relationship of distress and perceived control to coping with perceived racial discrimination among Black youth. Journal of Black Psychology, 31(3), 254-272.

Scott, W. A. \& Scott, R. (1989). Adaptation of immigrants: Individual differences and determinants. Oxford: Pergamon Press. 
* Sellers, R. M., Copleand-Linder, N., Martin, P. P., \& Lewis, R. (2006). Racial identity matters: The relationship between racial discrimination and psychological functioning in African American Adolescents. Journal of Research Adolescence, 16(2), 187-216.

* Sellers, R. M., \& Shelton, J. N. (2003). The role of racial identity in perceived racial discrimination. Journal of Personality and Social Psychology, 84(5), 1079-1092.

Selvarajah, C. (2004a). Equal employment opportunity: Acculturation experience of immigrant medical professionals in New Zealand in the period 1995 to 2000. Equal Opportunity International, 23, 50-73.

Selvarajah, C. (2004b). Expatriation experiences of Chinese immigrants in New Zealand: Factors contributing to adjustment of older immigrants. Management Research News, 27(8/9), 26-45.

Shaffer, M. A, Joplin, J. R. W., Bell, M. P., Lau, T., \& Oguz, C. (2000). Gender discrimination and job-related outcomes: A cross-cultural comparison of working women in the United States and China. Journal of Vocational Behavior, 57, 395-427.

Shore, L. M., \& Goldberg, C. B. (2005). Age discrimination in the workplace. In R. L Dipboye \& A.Colella (Eds). Discrimination at work: The psychological and organizational bases (pp203-225). New Jersey: Lawrence Erlbaum Associates, Inc.

Shore, L. M., Cleaveland, J. N., \& Goldberg, C. (2003). Work attitudes and decisions as a function of Manager Age and Employee Age. Journal of Applied Psychology, 88, 529-537.

* Shorey, H. S., Cowan, G., \& Sullivan, M. P. (2002). Predicting perceptions of discrimination among Hispanics and Anglos. Hispanic Journal of Behavioral Sciences, 24(1), 3-22.

Shutz, H., \& Six, B. (1996). How strong is the relationship between prejudice and discrimination? A meta-analytic answer. International Journal of Intercultural Relations, 20 (3/4), 441-462.

Sidanius, J., \& Pratto, F. (1999). Social dominance: An intergroup theory of social hierarchy an oppression. New York: Cambridge University Press. 
Siehl, C., \& Martin, J. (1988). Measuring organizational culture: Mixing qualitative and quantitative methods. In M. Jones, M. Moore, \& R. Snyder (Eds.), Inside organizations: Understanding the human dimension (pp. 79-104). Newbury Park, CA: Sage.

Sikorska-Simmons, E. (2005). Predictors of organizational commitment among staff in assisted living. The Gerontologist, 45(2), 196-205.

Slack, T., \& Jensen, L. (2002). Race, ethnicity and underemployment in nonmetropolitan America: A thirty-year profile. Rural Sociology, 67(2), 208-233.

* Slaughter, J. E., Bulger, C. A., \& Bachiochi, P. D. (2005). Black applicants' reactions to Affirmative Action plans: Influence of perceived procedural fairness, anticipated stigmatization, and anticipated remediation of previous injustice. Journal of Applied Social Psychology, 35(12), 2437 2476.

Smith, D. (2008). Globalization, Degradation and the Dynamics of Humiliation. Current Sociology, 56, pp. 371-379.

Smith, H., J., \& Ortiz, D. J. (2002). Is it just me? The different consequences of personal and group relative deprivation. In I. Walker, \& H. J. Smith (Eds.), Relative deprivation: Specification, development and integration. Cambridge: Cambridge University Press.

Smith, H., \& Walker, I. (2008). Feeling relative deprivation; The rocky road from comparison to actions. In L. Tropp, G. T. Finchilescu, \& U. Wagner (eds.). Emerging research directions for improving intergroup relations - Building on the legacy of Thomas F. Pettigrew (pp. 227243). Oxford: Blackwell Press.

Smith, M. L. (1980). Publication bias and meta-analysis. Evaluation in Education, 4, 22-24.

Smyth, J. M. (1998). Written emotional expressions: Effect sizes, outcome types, and moderating variables. Journal of Consulting and Clinical Psychology, 66, 174-184.

Solano, F. S., \& Kleiner, B. H. (2003). Understanding and preventing workplace retaliation. Management Research News, 26, 206-211.

* Son Hing, L. S., Bobocel, D. R., \& Zanna, M. P. (2002). Meritocracy and opposition to Affirmative Action: Making concessions in the face of 
discrimination. Journal of Personality and Social Psychology, 83(3), 493-509.

Sousa-Poza, A., \& Henneberger, F. (2004). Analyzing Job Mobility with Job Turnover Intentions: An International Comparative Study, Journal of Economic Issues, 38(1), 113-137.

Sowell,T. (2004). Affirmative action around the world: An empirical study. New Haven, CT: Yale University Press.

* Spencer-Rodgers, J., \& Collins, N. L. (2006). Risk and resilience: Dual effects of perceptions of group disadvantage among Latinos. Journal of Experimental Social Psychology, 42, 729-737.

Spoonley, P. (1993). Racism \& Ethnicity (2 ${ }^{\text {nd }}$ ed.). Auckland: Oxford University Press.

Statistics New Zealand. (2002a). People born overseas-: New Zealand Census of Population and Dwellings 2001. Wellington: Statistics New Zealand.

Statistics New Zealand. (2002b). 2001 Census: National Summary (Cultural Table). Retrieved on September 26, 2005 from http:www.stats.gov.nz.

Statistics New Zealand (2006a). 2006 Census: Quickstats of Population and Dwellings. Wellington: Statistics New Zealand. Retrieved on March 26, 2008 from www.stats.govt.nz/census

Statistics New Zealand (2006b). 2006 Census: Quickstats about Culture and Identity. Wellington: Statistics New Zealand. Retrieved on March 26, 2008 from www.stats.govt.nz/census

Stebbins, L. (2008). An investigation of individual job satisfaction as an outcome of individual perception of organizational culture. Dissertation Abstracts International, 69(1-A), 290.

Steeh, C., \& Krysan, M. (1996). Trends: Affirmative action and the public, 1970-1995. Public Opinion Quarterly, 60, 128-158.

Steenkamp, J-B.E.M. \& Baumgartner, H. (1998). Assessing measurement invariance in cross-national consumer research. Journal of Consumer Research, 25, 78-90.

Stewart, L D., \& Perlow, R. (2001). Applicant job, job status, and racial attitude as predictors of employment discrimination. Journal of Business and Psychology, 16(2), 259-275. 
Stewart, M. (2005). Racial discrimination of immigrants in employment selection of foremen in the engineering sector in New Zealand. Unpublished manuscript, Victoria University of Wellington, New Zealand.

Stone, E. F., Stone, D. L., \& Dipboye, R. L. (1992). Stigmas in organization: Race, handicaps, and physical attractiveness. In K. Kelley (Ed.), Issues, theory and research in industrial/organizational psychology (pp.385457). Amsterdam, Netherlands: Elsevier.

Stone-Romero, E. F. (2005). Personality-based stigmas and unfair discrimination in work organizations. In R. L. Dipboye \& A. Colella (Eds.), Discrimination at work: Psychological and organizational bases (pp. 37-61). New Jersey: Lawrence Earlbaum Associates, Inc.

Stroh, L. K., Brett, J. M., \& Reilly, A. H. (1996). Family structure, glass ceiling, and traditional explanations for the differential rate of turnover of female and male managers. Journal of Vocational Behaviour, 49, 99118.

Suarez-Orozco, M. M. (2005). Right moves? Immigration, globalization, utopia, and dystopia. In M. M. Suarez-Orozco, C. Suarez-Orozco \& D. B. Qin (Eds.), The new immigration: An interdisciplinary reader. New York: Taylor \& Francis Group.

Swidinsky R, Swidinsky M (2002) The relative earnings of visible minorities in Canada: new evidence from the 1996 census. Industrial Relations, 57, 630-660.

Swim, J. K., Cohen, L. L., \& Hyers, L. L. (1998). Experiencing everyday prejudice and discrimination. In J. Swim, \& C. Stangor (Eds.), Prejudice. The target's perspective (pp. 37-60). San Diego, CA: Academic Press.

Tacoli, C. (1999). International migration and the restructuring of gender asymmetries: Continuity and change among Filipino labor migrants in Rome. The International Migration Review, 33(3), 658 - 682.

Tajfel, H. (1978). Interindividual behaviour and Intergroup behaviour. In H. Tajfel (Ed.), Differentiation between social groups: Studies in the social psychology of intergroup relations. London: Academic Press.

Tajfel, H. (1981). Human groups and social categories. Cambridge: Cambridge University Press. 
Tajfel, H., \& Turner, J. C. (1979). An integrative theory of intergroup conflict. In W.G. Austin \& S. Worchel (Eds.), The social psychology of intergroup relations (pp. 33-47). Monterey, CA: Brooks/Cole.

Tajfel, H., \& Turner, J. C. (1986). The social identity theory of intergroup behaviour. In S. Worchel \& W. G. Austin (Eds.), Psychology of intergroup relations (pp. 7-24). Chicago: Nelson-Hall.

Taylor, D. M., Wright, S. C., Moghaddam, F. M., \& Lalonde, R. N. (1990). The personal/group discrimination discrepancy: Perceiving my group, but not myself, to be a target of discrimination. Personality and Social Psychology Bulletin, 16(2), 254-262.

Taylor, D. M., Wright, S. C., \& Porter, L. E. (1994). Dimensions of perceived discrimination: The personal/group discrimination discrepancy. In M. P. Zanna, \& J. M. Olson (Eds.), The psychology of prejudice: The Ontario symposium (Vol. 7., pp. 233-255). Hillside, NJ: Lawrence, Erlbaum Associates Inc.

Taylor, D. M., Wright, S. C., \& Ruggiero, K. M. (2001). The personal/group discrimination discrepancy: Responses to experimentally induced personal and group discrimination. The Journal of Social Psychology, 131(6), 847-858.

* Taylor, J., \& Turner, R. J. (2002). Perceived discrimination, social stress and depression in the transition to adulthood: Racial contrasts. Social Psychology Quarterly, 65(3), 213-225.

Teicher, J., \& Spearitt, K. (1995). From equal employment opportunity to diversity management: The Australian experience. International Journal of Manpower, 17, 109-133.

Ten Berge, J. M. F. (1986). Generalized approaches to the maxbet problem and the maxdiff problem, with application to canonical correlation. Psychometrika, 53(4), 487-494.

Terry, D. J. (2003). A social identity perspective on organizational mergers: The role of group status, permeability, and similarity. In s. A. Haslam, D. van Knippenberg, M. J. Platow \& N. Ellmers (Eds.), Social identity at work: Developing theory for organizational practice (pp. 223-240). Philadelphia, PA: Psychology Press. 
Therborn, G. (2006). Meaning, mechanisms, patterns, and forces: An introduction. In G. Therbon (Ed). Inequalities of the world: New theoretical framework, multiple empiricial approaches (1-58). UK: Bath Press.

Thomas, D. A., \& Alderfer, C. P. (1989). The influence of race on career dynamics: Theory and research on minority career experiences. In M. B. Arthur, D. T. Hall, \& B. S. Lawrence (Eds.), Handbook of career theory. Cambridge: Cambridge University Press.

Tienda, M., Donato, K. M., \& Cordero-Guzman, H. (1992). Schooling, color, and the labor force activity of women. Social Forces, 71, 365-395.

Tolbert-Coombs, A. A. \& King, R. K. (2005). Workplace discrimination: Experience of practicing physicians. Journal of the National Medical Association, 97, 467-470.

Tomkiewez, J., Adeyemi-Bello, T., \& Johnson, M. (1999). African Americans in business: Contrasting the attitudes of African American and white college business. Equal Opportunities International, 18(1), 19-26.

Tougas, F., \& Beaton, A. M. (1993). Affirmative action in the work place: For better or for worse. Applied Psychology: An International Review, 42, 253-264.

Tougas, F., \& Veilleux, F. (1990). The response of men to affirmative action strategies for women: The study of a predictive model. Canadian Journal of Behavioural Science, 22, 424-432.

Trentham, S., \& Larwood, L. (1998). Gender discrimination and the workplace: An examination of Rational Bias Theory. Sex Roles, 38(1/2), $1-28$.

Triandis, H. C., Kurowski, L. L. \& Gelfand, M. J. (1994). Workplace diversity. In H. C. Triandis, M. D. Dunnette, \& L. M. Hough (Eds.), Handbook of industrial and organizational psychology, Vol. $4\left(2^{\text {nd }}\right.$ ed., pp. 769-827). Palo Alto, CA: Consulting Psychologists Press.

Trlin, A., Henderson, A., \& North, N. (1999). Effects of unemployment among skilled immigrants from India. New Zealand Population Review, 25, 99-117.

Trlin, A., Henderson, A., \& North, N., Watts, N., \& Skinner, M. (2001). Immigration, human capital and productive diversity: Contrasts and 
issues in the employment experiences of new settlers from China, India and South Africa. A paper presented at Vibrant Voices and Visions for Ethnic New Zealand Conference, Massey University, Auckland, New Zealand.

Tsui, A. S., \& O’Reilly, C. A. (1989). Beyond simple demographic effects: The importance of relational demography in superior-subordinate dyads. Academy of Management Journal, 32, 402-423.

Tsui, A. S., Egan, T. D., \& O’Reilly, C. A. (1992). Being different: Relational demography and organizational attachment. Administrative Science Quarterly, 37, 547-579.

Turner, B. F. \& Turner, C. B. (1975). Race, sex, and perceptions of opportunity structure among college students. The Social Science Quarterly, 16, 345-360.

Turner, J. C. (1985). Social categorization and the self-concept: A social cognitive theory of group behaviour. In. E. J. Lawler (Ed.), Advances in group processes: Theory and research (Vol. 2, pp. 77-122). Greenwich, CT: JAI Press.

Turner, J. C., Hogg, M. A., Oakes, P. J., \& Wetherell, M. S. (1987). Rediscovering the social group: A self-categorization theory. Oxford: Blackwell.

Turner, J. C., Hogg, M. A., Turner, P. J., \& Smith, P. M. (1984). Failure and defeat as determinants of group cohesiveness. British Journal of Social Psychology, 23, 97-111.

van de Vijver, F. J. R., \& Leung, K. (1997). Methods and data analysis of comparative research. Thousand Oaks, CA: Sage.

Van Hemert, D. A. (2003). Cross-cultural meta-analysis. In W. J. Lonner, D. L. Dinnel, S. A. Hayes, \& D. N. Sattler (Eds.), Online readings in pychology and clture (Unit 2, Chapter 13), (http://www.wwu.edu/ culture), Center for Cross-Cultural Research, Western Washington University, Bellingham, Washington U.S.A.

van Knippenberg A., \& Ellemers, N. (1993). Strategies in intergroup relations. In M. A. Hogg \& D. Abrams (Eds), Group motivation: Social psychological perspectives (pp. 17-23). New York: Prentice Hall. 
van Knippenberg A., \& van Oers, H. (1984). Social identity and equity concerns in intergroup perceptions. British Journal of Social Psychology, 23, 351-361.

Van Muijen, J. J., Koopman, P., De Witte, K., De Cock, G., Susanj, Z., Lemoine, C., Bourantas, D., Papalexandris, N., Branyicski, I., Spaltro, E., Jesuino, J., Gonzalves das Neves, J., Pitariu, H., Konrad, E., Peiro, J., Gonzalez-Roma, V., \& Turnipseed, D. (1999). Organizational culture: The focus questionnaire. European Journal of Work and Organizational Psychology, 8, 551-568.

Van Oudenhoven, J. P., Ward, C., \& Masgoret, A-M. (2006). Patterns of relations between immigrants and host societies. International Journal of Intercultural Relations, 30, 637-651.

Velicer, W. F. \& Fava, J. L. (1998). Effects of variable and subject sampling on factor pattern recovery. Psychological Methods, 3, 231-251.

Verkuyten, M. (1998). Perceived discrimination and self-esteem among ethnic minority adolescents. Journal of Social Psychology, 138, 479-493.

Verkuyten, M. (1993). Self-esteem among ethnic minorities and three principles of self-esteem formation: Turkish children in the Netherlands. International Journal of Psychology, 28(3), 307-321.

Verkuyten, M. (2003a). Ethnic in-group bias among minority and majority early adolescents: The perception of negative peer behaviour. British Journal of Developmental Psychology, 21, 543-565.

Verkuyten, M. (2003b) Discourses about ethnic group (de-)essentialism: oppressive and progressive aspects. British Journal of Social Psychology, 42, 371-391.

Verkuyten, M. (2003c) Positive and negative self-esteem among ethnic minority early adolescents: Social and cultural sources and threats. Journal of Youth and Adolescence, 32, 267-277.

*Verkuyten, M., \& Brug, P. (2002). Ethnic identity achievement, self-esteem, and discrimination among Surinamese adolescents in the Netherlands. Journal of Black Psychology, 28(2), 122-141.

Virdee, S. (1995). Racial violence and harassment. The Hospital Anxiety and Depression, 11, 227-234. 
Viswesvaran, C., Sanchez, J. I., \& Fisher, J. (1999). The role of social support in the process of work stress: A meta-analysis. Journal of Vocational Behaviour, 54, 314-334.

Walker, I., \& Mann, L. (1987). Unemployment, relative deprivation, and social protest. Personality and Social Psychology Bulletin, 13, 275-283.

Walrond, C. (2007). 'Africans', Te Ara - the encyclopedia of New Zealand. Retrieved on March 30, 2007, from http://www.TeAra.govt.nz/ NewZealanders/ NewZealandPeoples/Africans/en.

Ward, C., Bochner, S., \& Furnham, A. (2001). The psychology of culture shock ( $2^{\text {nd }}$ ed). UK: Routledge.

Ward, C., \& Leong, C.-H. (2006). Intercultural relations in plural societies. In D. L. Sam \& J. W. Berry (Eds.), The Cambridge handbook of acculturation psychology (pp. 484-503). Cambridge, UK: Cambridge University Press.

Ward, C., \& Masgoret, A-M. (2004). Responses to immigrants seeking employment: A case of discrimination? Unpublished manuscript, Victoria University of Wellington, New Zealand.

Ward, C., \& Masgoret, A.-M. (2007). Immigrant entry into the workforce: A research note from New Zealand. International Journal of Intercultural Relations, 31(4), 525-520.

Ward, C., Fischer, R., Lam, F. S. Z., \& Hall, L. (2009). The convergent, discriminant, and incremental validity of scores on a self-report measure of cultural intelligence. Educational and Psychological Measurement, 69, 85-105.

Wasti, S.A. (2003). The influence of cultural values on antecedents of organizational commitment: An individual-level analysis. Applied Psychology: An International Review, 52: 533-554.

Waters, H., Jr. (1994). Decision-making and race. International Journal of Intercultural Relations, 18, 449-467.

Waters, M. C., \& Jimenez, T. R. (2005). Assessing immigrant assimilation: New empirical and theoretical challenges. Annual Review of Sociology, $31,105-25$.

* Watts, R. J., \& Carter, R. T. (1991). Psychological aspects of racism in organization. Group \& Organization Studies, 16(3), 328-344. 
Way, N. (1997). Adult and peer discrimination measure. Unpublished document.

Weick, K. (1995). Sensemaking in organizations. Thousand Oaks, CA: Sage.

Weinberg, S. B. (1998). Mexican American mothers and the welfare debate: A history of exclusion. Journal of Poverty, 2(3), 53-75.

Weiss, J. (1983). The New Zealand multi-cultural workforce. In N. R. Marsh \& W.F. McDonald (Eds.), The multi-cultural workforce in New Zealand and Australia. Auckland: University of Auckland.

Westerman, J. W., \& Yamamura, J. H. (2007). Generational preferences for work environment fit: Effects on employee outcomes. Career Development International, 12(2), 150-161.

Westhoff, W. (1993). A psychological study of albinism in a predominantly mullato Caribbean community. Psychological Reports, 73, 1007- 1010.

Westwood, R. I. (1992). Organizational behavior: Southeast Asian perspectives. Hong Kong: Longman Group (Far East) Ltd.

Wilcox, C. (1997). Racial and gender consciousness among African American women: Sources and consequences. Women and Politics, 17(1), 73-94.

Williams, D. R. (1997). Race and health: Basic questions, emerging directions. Annals of Epidemiology, 7, 322-33.

Williams, D. R., Neighbors, H. W., \& Jackson, J. S. (2003). Racial/ethnic discrimination and health: Findings from community studies. American Journal of Public Health, 93, 200-208.

Williams, D. R., Spencer, M. J., \& Jackson, J. (1999). Race, stress and physical health: The role of group identity. In R. J. Contrada \& R. D. Ashmore (Eds.), Self, Social Identity, and Physical Health, (pp. 71-100). New York: Oxford University Press.

Williams, D. R., Yu, Y., Jackson, J. S., \& Anderson, N. B. (1997). Racial differences in physical and mental health: Socioeconomic status, stress and discrimination. Journal of Health Psychology, 2, 335-351.

Wilson, M G., Gahlout, P., Mouly, S. \& Liu, L. (2005). Three strikes and you're out: The impact of ethnicity, ethnicity of name and immigrant status on access to employment. Unpublished manuscript, The University of Auckland, New Zealand. 
Wilson, R. (1988). Think about our rights: Civil liberties in the United States. New York, NY: Walker.

Winter-Ebner, R. (1994). Motivation for migration and economic success. Journal of Economic Psychology, 15, 269-284.

Wong, Y. T., Ngo, H. Y., \& Wong, C. S. (2002). Affective organizational commitment of workers in Chinese joint ventures. Journal of Managerial Psychology, 17 (7), 580-598.

Wright, S. C., Taylor, D. T., \& Moghaddam, F. M. (1990). Responding to membership in a disadvantaged group: From acceptance to collective protest. Journal of Personality and Social Psychology, 58, 994-1003.

Yao, X., \& Wang, L. (2006). The predictability of normative organizational commitment for turnover in Chinese companies: a cultural perspective. International Journal of Human Resource Management, 17 (6), 10581075 .

Yearta, S. K., \& Warr, P. (1995). Does age matter? The Journal of Management Development, 14, 28-35.

Ying, Y. (1996). Immigration satisfaction of Chinese Americans: An empirical examination. Journal of Community Psychology, 24, 3-16.

Zenger, T., \& Lawrence, B. (1989). Organizational demography: The differential effects of age, and tenure distributions on technical communication. Academy of Management Journal, 32, 353-376.

* Zlobina, A., Basabe, N., Paez, D., \& Furnham, A. (2006). Sociocultural adjustment of immigrants: Universal and group-specific predictors. International Journal of Intercultural Relations, 30(2), 195-211. 


\section{Appendix A}

\section{Focus Group Discussion Schedule}

\section{Topic: Immigrants’ Experiences in the NZ workplace}

Part 1:

Introduction (state objective of the study, procedure of the discussion and ground rules, questions from participants regarding the research)

Part II: Discussion

9. Can you share your reasons for moving to New Zealand? [Probe - What were your expectations in terms of advantages and disadvantages in you're a) profession b)income, c) family life]

10. How did you apply for work in NZ?

- What happened during the application process?

11. In your workplace, can you please describe your

- job assignment or the task that your doing in comparison to your qualification

- methods of payment and rewards that you receive

- $\quad$ benefits that you enjoy

- training or further skill development opportunities

12. In your workplace, can you please describe your relationships with

- $\quad$ peers

- immediate supervisors

- $\quad$ higher superiors

13. Can you recall any experience that hindered/helped you in

- feeling at ease with your job

- feeling at ease with your co-workers

14. How did these experiences affect your work and your commitment to the organization?

15. In which way your experiences at work affected your long-term plans?

16. Overall, how would you describe the way your organization deal with immigrants or people from different ethnicity?

Part III:

Post Discussion (Saying thanks, reiterating confidentiality, debriefing) 


\section{Appendix B}

\section{Perceptions of Discrimination Scales - Initial Items During Item Development Stage}

\section{Scale 1: Job-Entry Perceptions of Discrimination}

(Personal Characteristics)

1. My name is a hindrance in getting a job.

2. My organization prefers to hire local New Zealanders.

3. I was denied a job because of my looks.

4. Another applicant with fewer qualifications got the job because my looks are different from majority of the workforce.

5. My cultural background is a limitation in obtaining a job in New Zealand

6. People of my background don't get full-time job positions.

(Educational Background)

7. I am employed according to my educational qualifications gained overseas.

8. My educational background is recognized in my current position.

9. My educational qualifications gained overseas were recognized in my job.

10. I need to obtain local qualifications just to obtain the job that I want in New Zealand.

11. My educational training and skills are not recognized by NZ authorities.

(Professional/Work Experience)

12. I didn't get a job because I lack work experience specific to NZ

13. I am employed according to my professional/work experience gained overseas.

14. My professional experience was recognized in my current position.

15. I got a job relevant to my work experience but low in terms of position level.

16. I got a job different from my previous work experience.

17. My skills gained from previous work experience overseas are utilized in my job.

(Language/accent)

18. I was denied a job because I am not a native English Speaker.

19. My accent is a limitation at obtaining work.

20. English-native speakers have better access to jobs.

21. Having English as a second language is a barrier to enter the workforce (Networking/Knowing people)

22. Recruitment consultants/agencies are helpful in finding a job for people of my background.

23. Recruitment agencies provide equal support to people of different ethnic backgrounds.

24. Networking is helpful in obtaining a job in New Zealand.

25. It is easy to get a job in New Zealand if you know people in an organization.

26. References are a necessity in finding a job in New Zealand.

\section{Scale 2: On-the-Job Perceptions of Discrimination (Formal Structures)}

(Promotions/Developmental)

27. I have opportunities for advancement.

28. My opportunity for advancement is somewhat limited.

29. I am in a dead-end job.

30. I have a good chance for promotion.

31. My promotion at this organization has been regular.

32. There is a clearly visible career line of increasing rewards and promotion for me in this organization. 
33. I feel my training opportunities are fair considering what other people in this firm are paid.

34. I have been unfairly passed over for special skills and developmental trainings.

(Salary/Pay)

35. I feel my salary/wage is fair considering what other people in this firm are paid.

36. If feel my salary/wage is fair considering what other firms in this sector pay.

37. I get lower pay compared to local New Zealanders.

38. My salary/wage is not commensurate to the amount of work I do in this organization.

(Fringe Benefits)

39. I feel my fringe benefits are fair considering the same benefits provided by other firms in this area.

40. I get health insurance and other wellness benefits comparable to other employees in my organization.

41. I have paid holiday leave entitlements comparable to other employees in my organization.

42. I have paid sick leave entitlement similar to other employees in my organization.

(Overall)

43. This organisation provides an atmosphere of equal opportunity for everyone regardless of ethnic background.

44. This organisation's policies and procedures are unfair to people of my ethnic background.

45. I feel that this organisation considers the needs of all employees regardless of their cultural background.

Scale 3: On-the-Job Perceptions of Discrimination (Informal Structures)

(General)

46. At work, I feel uncomfortable when others make jokes or negative commentaries about people of my ethnic background.

47. At work, I feel that others exclude me from their activities because of my ethnic background.

48. Insulting jokes or comments are directed towards me because of my cultural background.

49. At work, many people have negative stereotypes about my culture or ethnic (Peers) group and treat me as if they were true.

50. I have excellent working relationships with colleagues regardless of our cultural background.

51. I experience frequent conflict with my co-workers on the job because I come from a different culture.

52. My contributions at work are recognized by my peers although I am different from them.

(Supervisors)

53. I have excellent working relationships with supervisors regardless of my ethnic background.

54. I experience frequent conflict with my superior(s) in my organization because I come from a different culture.

55. My contributions at work are recognized by my supervisor even if I come from a different cultural background. 


\section{Appendix C}

\section{Immigrants' Perceptions of Discrimination in the Workplace Scales (IPDWS) - Pilot Version}

\section{Scale 1 - ABOUT FINDING WORK}

\section{PD: Hiring Policies/Practices/Preferences}

1. My name is a hindrance in getting a job.

2. My educational qualifications gained overseas were recognized in my job.

3. Recruitment consultants/agencies are helpful in finding a job for people of my ethnic background

4. I was denied a job because of my looks.

5. My educational background is recognized in my current position.

6. It is easy to get a job in New Zealand if you know people in that organization.

7. I was denied a job because I am not a native English speaker.

8. I was hired according to my educational qualifications.

9. My organization prefers to hire local New Zealanders.

10. English-native speakers have better access to jobs.

11. I didn't get a job because I lack local work experience.

12. Networking is helpful in obtaining a job in New Zealand.

13. My accent is a barrier to obtaining employment.

14. My skills gained from previous work experience overseas are utilized in my job.

15. Having English as a second language is a barrier to enter the workforce.

16. I got a job relevant to my work experience but low in terms of position level.

17. I was denied a desired position due to my accent.

18. I need to obtain local qualifications to obtain the job I want.

19. I got a job different from my previous work experience gained overseas.

20. Contact persons in an organization can help you obtain a job.

21. I am employed according to my professional/work experience gained overseas.

22. Another applicant with fewer qualifications got the job because my looks are different from majority of the workforce.

23. My educational training and skills are not recognized by NZ authorities.

24. References are a necessity in finding a job in New Zealand.

\section{Scale 2 - ABOUT WORKING CONDITIONS}

PD: Policies - Promotions

25. I have opportunities for advancement.

26. My opportunity for advancement is somewhat limited.

27. I am in a dead-end job

28. I have a good chance for promotion

29 . My promotion at this organization has been regular.

30 . There is a clearly visible career line of increasing rewards and promotion for me in this organization

\section{PD: Policies - Pay \& Fringe Benefits}

31. I feel may salary/wage is fair considering what other people in this firm are paid.

32. My salary/wage is not commensurate to the amount of work I do in this organization

33. I have paid holiday leave entitlements comparable to other employees in my organization

34. I have paid sick leave entitlement similar to other employees in my organization

35. I get health insurance and other benefits comparable to other employees in my organization.

36. If feel my salary/wage is fair considering what other firms in this sector pay.

37. My fringe benefits (e.g. leave entitlements, insurance) are not commensurate to what I should get in my line of work.

38. I get paid according to my level of training.

39. I get health insurance and other benefits comparable to other firms in this sector. 
40. I get paid comparable to other employees in my organization.

41. The compensation I get is low considering the tasks involved in my work.

\section{PD: Policies - Trainings}

42. I feel may training opportunities are fair considering the trainings provided for other employees in this organization.

43. I have been unfairly passed over for special skills and developmental trainings.

44. My training opportunities are adequate considering what other people get in the same position.

45. My training opportunities are limited in this organization.

46. I get the appropriate trainings that are necessary for my development.

\section{PD: Organizational Policies in General}

47. This organisation provides an atmosphere of equal opportunity for everyone

48. This organisation's policies and procedures are unfair to people of my background.

49. I feel that this organisation considers the needs of all employees regardless of their cultural background.

50. Policies and procedures of this organization are applicable only to local New Zealanders.

51. My organization thinks about the welfare of every employee regardless of their ethnic background.

\section{Interaction - General}

\section{Scale 3 - ABOUT RELATIONSHIPS AT WORK}

52. At work, I feel uncomfortable when others make jokes or negative commentaries about people of my ethnic background.

53. My contributions at work are recognized by anyone although I am different from them.

54. I have excellent working relationships with everyone regardless of our cultural backgrounds.

55. At work, I feel that others exclude me from their activities because of my ethnic background.

56. At work, many people have negative stereotypes about my culture or ethnic group and treat me as if they were true

\section{Interaction - Peers}

57. At work, I feel uncomfortable when my co-workers make joke or negative commentaries about my ethnic background.

58. I have excellent working relationships with co-workers regardless of our cultural backgrounds.

59. I experience frequent conflict with my co-workers on the job because I come from a different culture.

60. At work, I feel that my co-workers exclude me from their activities because of my ethnic background.

61. My contributions at work are recognized by my co-workers although I am different from them.

Interaction - Superiors

63. I have excellent working relationships with superiors regardless of my ethnic background.

64. At work, I feel uncomfortable when my supervisor makes jokes or negative commentaries about people of my ethnic background.

65. At work, my superior have negative stereotypes about my culture or ethnic group and treat me as if they were true

66. I experience frequent conflict with my supervisor in my organization because I come from a different culture.

67. My contributions at work are recognized by my superiors even if I come from a different cultural background. 


\section{Appendix D}

\section{Employee Perceptions of Discrimination in the Workplace Scales (EPDWS) - Initial Items}

\section{Scale 1 - FINDING WORK}

1. I was hired according to my educational qualifications.

2. This organization denies jobs to people whose physical appearance is different from the majority.

3. My educational background is recognized in my current position.

4. It is easy to get a job in if you know people in this organization.

5. A person is denied a job in this organization if she/he is a non-native English speaker.

6. Networking is helpful in obtaining a job in my organization.

7. My educational qualifications are recognized in my job.

8. Having an accent is a barrier to obtaining employment in this organization.

9. My job uses the skills I gained from previous work experience.

10. A person needs to obtain further qualifications to get a job he/she wants in this organization.

11. Having contact persons can help you obtain a job in this organization.

12. I am employed according to my professional/work experience.

13. Qualifications matter more than physical appearance in this organization.

14. This organization considers only the trainings/skills that are certified by NZ qualification authorities.

\section{Scale 2 - WORK CONDITIONS}

15. I have opportunities for advancement.

16. I feel my salary/wage is fair considering what other people in this firm are paid.

17. Policies and procedures of this organization are applicable only to local New Zealanders.

18. If feel my salary/wage is fair considering what other firms in this sector pay.

19. Fringe benefits (e.g. leave entitlements, insurance) in this organization are not commensurate to the employees' line of work.

20. This organisation's policies and procedures are fair to all employees regardless of their ethnic background.

21. I have a good chance for promotion.

22. I am in a dead-end job.

23. I get health insurance and other benefits comparable to other firms in this sector.

24. My salary/wage is not commensurate to the amount of work I do in this organization.

25. My promotion at this organization has been regular.

26. This organization thinks about the welfare of every employee regardless of their ethnic background.

27. Paid sick leave entitlement given by this organization is comparable to other similar sectors.

28. There is a clearly visible career line of increasing rewards and promotion for me in this organization.

\section{Scale 3 - WORK RELATIONSHIPS}

29. I have excellent working relationships with co-workers regardless of my cultural/ethnic background.

30. At work, my supervisor makes jokes or negative comments about people of my cultural/ethnic background.

31. My contributions at work are recognized by my supervisor regardless of my cultural/ethnic background.

32. I have excellent working relationships with supervisors regardless of my cultural/ethnic background.

33. My contributions at work are recognized by my co-workers regardless of cultural/ethnic background.

34. At work, my co-workers make joke or negative comments about people of my

cultural/ethnic background. 


\title{
Appendix E
}

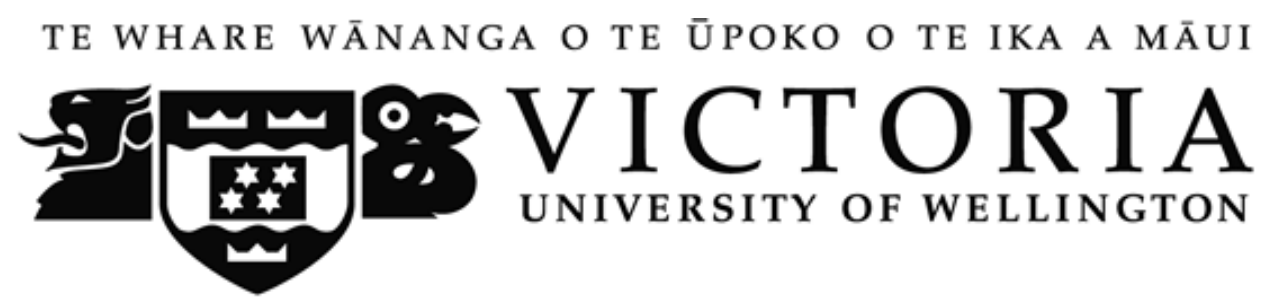

\author{
Information Sheet - Study 2 (Focus Groups Discussion) \\ Topic: Immigrants' Experiences in the NZ workplace
}

\section{Who is conducting the research?}

- I am Soc Diego, a Psychology PhD student at Victoria University of Wellington. My study is on immigrants' experiences in the NZ workplace. Dr. Astrid Podsiadlowski, Dr. Colleen Ward and Dr. Ronald Fisher are supervising my work.

\section{What is the purpose of this research?}

- This research is interested in your workplace experiences as first generation skilled immigrants employed in various NZ industries. It is our expectation that upon arrival, one of your primary objectives is to find a suitable job which could help you earn a living. Thus, we are interested on how immigrants manage to obtain their jobs taking into account personal qualifications, skills and work experiences they had before coming to NZ. We also want to explore current issues relating to immigrants' interactions with local New Zealanders and other migrant groups in a growing multi-cultural work environment. Hence, it would be interesting to learn about your work experiences in New Zealand..

\section{What is involved if you agree to participate?}

- If you agree to participate, you will join a small group (3-5 persons) who will be discussing experiences in the workplace specifically in dealing with people from different cultural backgrounds, experiences from finding a job to keeping the job, current job assignments, interactions with co-workers, and other job-related experiences.

- With your permission, the discussions will be audio-taped and be transcribed later on.

- The discussions will take no more than two hours. You are free to withdraw at any point up until the completion of the discussion, and the data pertaining to you will not be included in the transcriptions.

- It is important that anything shared or discussed today is not repeated outside of this group.

- As a token of our appreciation, we will give you $\$ 10$ grocery vouchers at the end of the focus group discussions.

\section{Privacy and Confidentiality}

- During transcriptions, we will replace your names and pertinent details by codes so that no data will be directly traceable to you. Hence, you will not be directly identified nor be pinpointed to your employers and the immigration service. Accordingly, you will not be directly identified in the research results, publications or presentations.

- We assure you that no personal information about you as a participant will be sent to your employers or NZ immigration.

- Only I and my supervisors will have direct access to your coded data. Per requirements of some scientific journals and organisations, your coded data may be shared with other competent professionals.

- A copy of the coded data will remain in the custody of Dr. Colleen Ward for at least 5 years. 


\section{What happens to the information that you provide?}

- Together with other data, the results of this research will be a part of my thesis. Overall results of this research may also be published in scientific journals or be presented at scientific conferences.

\section{Feedback}

Results of this study will be available by approximately 31 May 2008. Please indicate your email address or postal address if you want us to notify you regarding the availability of the results or the presentation materials. Alternatively, results may also be viewed via the CACR webpage in PDF-format at http://www.vuw.ac.nz/cacr/.

If you have any further questions regarding this study, please contact me at Soc.Diego@vuw.ac.nz, phone (04) 463-6976, postal address: School of Psychology, Victoria University. PO Box 600, Wellington, or any of my supervisors at:

Astrid.Podsiadlowski@vuw.ac.nz, phone (04) 463-7491

Colleen.Ward@vuw.ac.nz, phone (04) 463-6976

Ronald.Fischer@vuw.ac.nz, phone (04) 463-6548

Thank you for your participation.

Sincerely yours,

Soc Diego

\section{Statement of consent}

I have read the information about this research and any questions I wanted to ask have been answered to my satisfaction.

I agree to participate in this research.

I give my consent to participate in the focus groups discussions that will be audio-taped and later on transcribed.

I understand that I can withdraw my consent at any time and any information or data I have given will not be included in the research.

Name:

Signature:

Date:

I would like a copy of the summary of the results of this study. YES / NO (If yes, please indicate email address or postal address below)

Email Address:

or, Postal Address:

Copy to:

[a] participant,

[b] researcher (initial both copies below) 


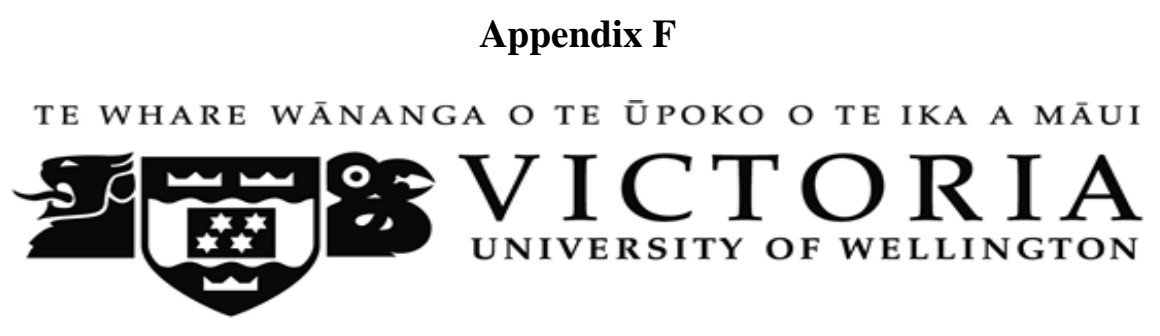

Information Sheet - Study 3

(Immigrants’ Perceptions Survey)

I'm Soc Diego, a PhD Student in the School of Psychology at Victoria University of Wellington, and my study is on immigrants' perceptions of the New Zealand workplace. Dr. Ronald Fischer, Dr. Astrid Podsiadlowski, and Professor Colleen Ward are supervising my work.

\section{Purpose of this study}

As an immigrant to New Zealand, your views about your workplace provide valuable information on how well you are adapting in an environment different from your home country. We are interested in your perceptions about the various aspects of your work and your organization. We also aim to understand what is happening in New Zealand organizations by looking into what individual employees think specifically on the following areas:

- your general feelings about your organization's practices and procedures

- your interactions with co-workers and supervisors

- some similarities and differences in your work details and experiences compared to other employees across different types of organizations

The results will help us understand the experiences of different immigrant groups across different organizations in New Zealand. From the overall findings, we aim to present useful feedback to organizations and to policy making bodies for improving procedures specifically related to programs that will assist migrants in their successful settlement in New Zealand.

\section{What happens if you agree to participate?}

This research is voluntary and you are not required to be involved. If you choose to participate, please answer the attached questionnaire which will take you approximately $30-45$ minutes to complete. Please do not write your name or any identifying information on the questionnaire itself. We are providing you with a pre-stamped envelope so you can directly mail us your completed survey. We would appreciate if you could return the completed survey by the end of November 2006.

\section{What happens to the information you provide?}

All your responses will remain completely anonymous. Any response information will be identified by a code number only and at no point will you be personally identified. Further, your anonymous response will be added to 
those of other participants. The group data may be analyzed and written up for presentations, professional publications and as part of my $\mathrm{PhD}$ thesis. The anonymous data will be kept in Prof. Ward's office for at least 5 years after publication, and the electronic data will be shared with other competent researchers upon request.

\section{$\underline{\text { Feedback }}$}

Results will be available by approximately May 31, 2008. If you want to be informed on the availability of the result summary or presentation materials, please write down your contact details on the feedback sheet and return it using the separate mailing envelope. That way we cannot link your completed survey to your return address. Alternatively, overall results can also be viewed via the CACR webpage in PDF-format at http://www.vuw.ac.nz/cacr/.

If you have any further questions regarding this study, please contact me via email: Soc.Diego@vuw.ac.nz, phone (04) 463-6976 or any of my supervisors: Ronald.Fischer@vuw.ac.nz, phone Astrid.Podsiadlowski@vuw.ac.nz, phone 463-6548, Colleen.Ward@,vuw.ac.nz, phone (04) 463-6976. (04) 463-7491, and

Thank you. 


\section{Appendix G}

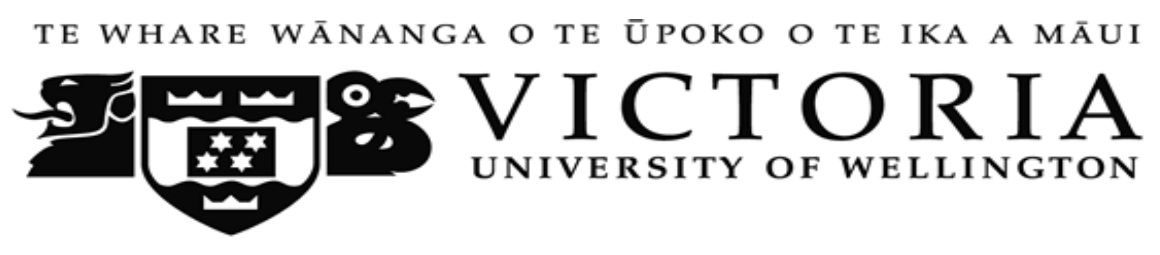

Information Sheet - Study 4

\section{Dear Participant,}

I am a student in Organizational Psychology at Victoria University Wellington. We would like to invite you to participate in a study on your views and perceptions of your work place. This is part of a study project under supervision by Dr Ronald Fischer. It is part of an international research programme where we aim to understand how organizations function in different countries. The study also aims to look into how employees of different cultural backgrounds perceive various aspects of their job and their organization. Your help is very much appreciated because it will help us to obtain a better understanding of the way people think about their work environment. This information can then be used for training programmes and Human Resource Development initiatives. Even if you don't feel very positively about your work, your perceptions are important and will help us to improve working conditions.

We would appreciate if you could fill in the following questionnaire. Your participation in this study is entirely optional and you are under no obligation to respond. By completing and returning the questionnaire you agree that the data will be used and analysed.

This questionnaire should take you no longer than 30 minutes. Your responses, as well as your personal identity will remain completely anonymous. Your response information will be identified by coded number only and at no point will you be personally identified. Please do not write your name or any identifying information on the questionnaire itself. By completing and returning this questionnaire you acknowledge permission for your responses to be analysed. Some of the data might be used in professional publications or presentations.

On the next few pages, we ask you some more questions about your perceptions of practices in your organization as well as your interactions in your workplace. Please answer the questions using the provided rating scales or ticking the appropriate box. For example, in rating the scales, circle 2 if you somewhat agree with the statement that people needs more free parking. Please remember, there are no right or wrong answers.

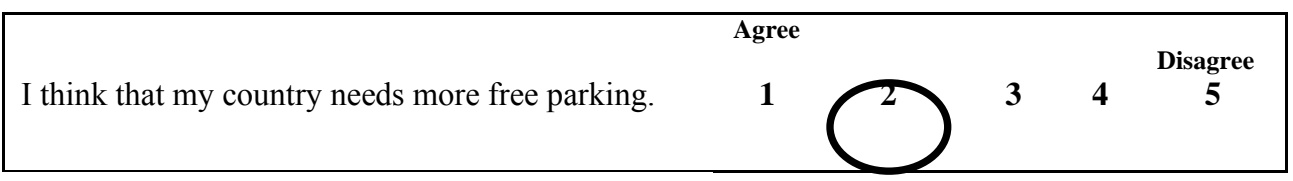

For more information, please do not hesitate to contact Dr Ronald Fischer, Victoria University of Wellington, New Zealand: e-mail: Ronald.Fischer@vuw.ac.nz

Tel. 044636548

Thank you very much for your help and cooperation. 